\title{
GROUND-WATER QUALITY NEAR A SEWAGE-SLUDGE RECYCLING SITE AND A LANDFILL NEAR DENVER, COLORADO
}

U. S. GEOLOGICAL SURVEY

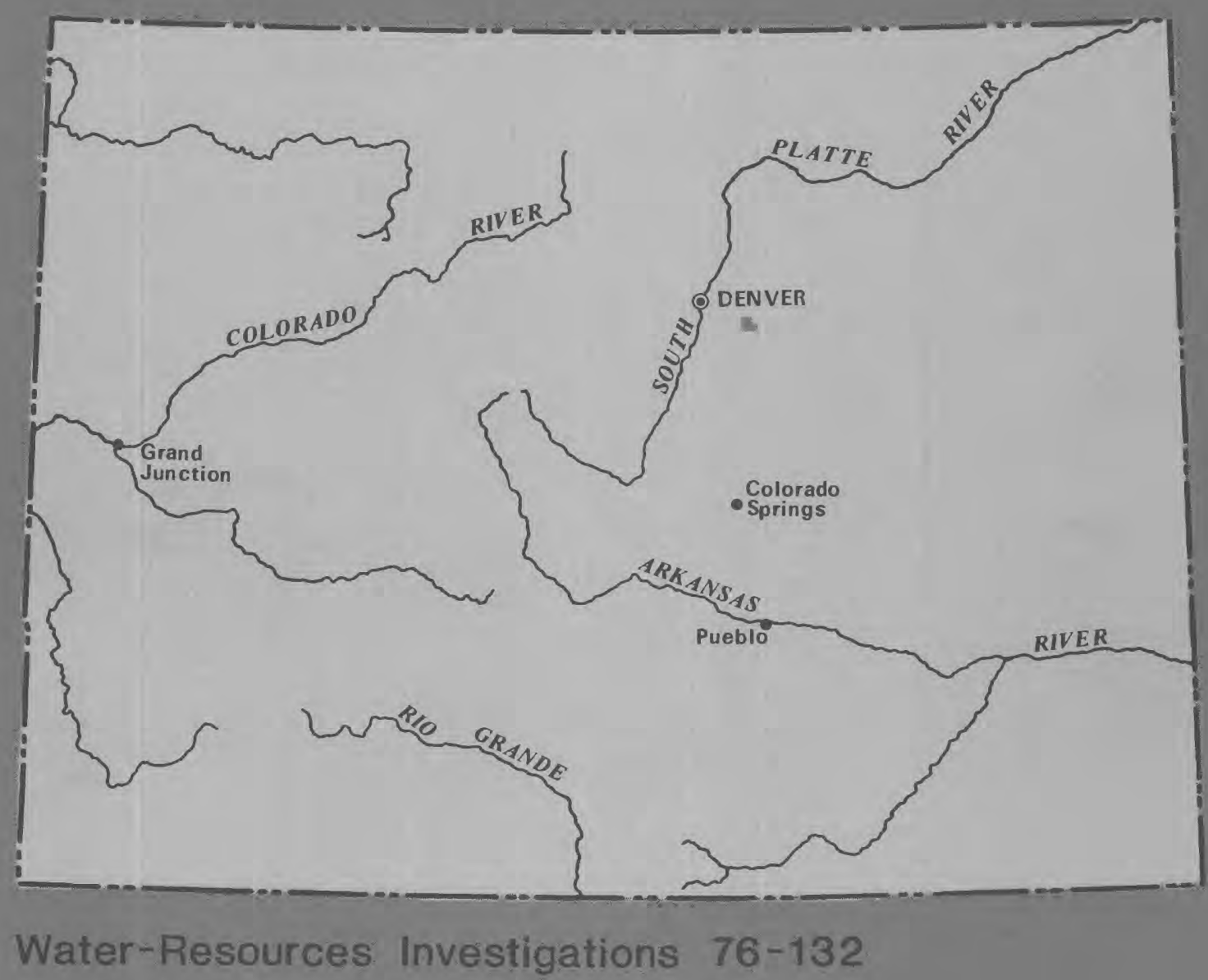

Prepared in cooperation with the Metropolitan Denver Sewage Disposal District and the Colorado Geological Survey 


\begin{tabular}{|c|c|c|}
\hline $\begin{array}{l}\text { BIBLIOGRAPHIC DATA } \\
\text { SHEET }\end{array}$ & 1. Report No. & 3. Recipient's Accession No. \\
\hline \multirow{2}{*}{\multicolumn{2}{|c|}{$\begin{array}{l}\text { 4. Title and Subtitle } \\
\text { GROUND-WATER QUALITY NEAR A SEWAGE-SLUDGE RECYCLING SITE } \\
\text { NEAR DENVER, COLORADO }\end{array}$}} & $\begin{array}{l}\text { 5. Report Date } \\
\text { May } 1977 \\
\end{array}$ \\
\hline & & 6. \\
\hline \multicolumn{2}{|l|}{$\begin{array}{l}\text { 7. Author(s) } \\
\text { S. G. Robson }\end{array}$} & $\begin{array}{l}\text { 8. Performing Organization Rept. } \\
\text { No. USGS/WRI 76-132 }\end{array}$ \\
\hline \multicolumn{2}{|c|}{$\begin{array}{l}\text { 9. Performing Organization Name and Address } \\
\text { U.S. Geological Survey, Water Resources Division } \\
\text { Box } 25046 \text { Denver Federal Center, Mail Stop } 415 \\
\text { Lakewood, Colorado } 80225\end{array}$} & \begin{tabular}{|l} 
10. Project/Task/Work Unit No. \\
11. Contract/Grant No.
\end{tabular} \\
\hline \multirow{2}{*}{\multicolumn{2}{|c|}{$\begin{array}{l}\text { 12. Sponsoring Organization Name and Address } \\
\text { U.S. Geological Survey, Water Resources Division } \\
\text { Box } 25046 \text { Denver Federal Center, Ma11 Stop } 415 \\
\text { Lakewood, Colorado } 80225\end{array}$}} & $\begin{array}{l}\text { 13. Type of Report \& Period } \\
\text { Covered } \\
\text { Final }\end{array}$ \\
\hline & & 14. \\
\hline
\end{tabular}

\section{Supplementary Notes}

Prepared in cooperation with the Metropolitan Denver Sewage Disposal District and the Colorado Geological Survey

16. Abstracts

The Metropolitan Denver Sewage Disposal District and the City and County of Denver operate a sewage-sludge recycling site and a landfill in an area about $15 \mathrm{miles}$ (24 kilometers) east of Denver. The assessment of the effects of these facilities on the ground-water system indicated that five wells perforated in alluvium were found to have markedly degraded water quality. One well was located in the landfill and water that was analyzed was obtained from near the base of the buried refuse, two others were located downgradient and near sewage-sludge burial areas, and the remaining two are located near stagnant surface ponds. Concentrations of nitrate in wells downgradient from fields where sludge is plowed into the soil were higher than background concentrations due to the effects of the sludge disposal. No evidence of water-quality degradation was detected in deeper wells perforated in the bedrock formations.

17. Key Words and Document Analysis. 17a. Descriptors

*Landfi11, *Sewage sludge, *Water chemistry, *Water-pollution sources

17b. Identifiers/Open-Ended Terms

Arapahoe County, Colorado, *Denver Formation, *Ground-water movement, Ground-water-quality degradation

17c. COSATI Field/Group

18. Availability Statement

No restriction on distribution

\begin{tabular}{|l|c|}
\hline $\begin{array}{l}\text { 19. Security Class (This } \\
\text { Report) } \\
\text { UNCLASSIFIED }\end{array}$ & $\begin{array}{c}\text { 21. No. of Pages } \\
142\end{array}$ \\
\hline $\begin{array}{l}\text { 20. Security Class (This } \\
\text { Page } \\
\text { UNCLASSIFIED }\end{array}$ & 22. Price \\
\hline
\end{tabular}


GROUND-WATER QUALITY NEAR A SEWAGE-SLUDGE RECYCLING SITE

AND A LANDFILL NEAR DENVER, COLORADO

By S. G. Robson

U.S. GEOLOGICAL SURVEY

Water-Resources Investigations 76-132

Prepared in cooperation with the

Metropolitan Denver Sewage Disposal District and the

Colorado Geological Survey 


\section{UNITED STATES DEPARTMENT OF THE INTERIOR}

CECIL D. ANDRUS, Secretary

GEOLOGICAL SURVEY

V. E. McKelvey, Director

For additional information write to:

District Chief

U.S. Geologica1 Survey

Water Resources Division, Colorado District

Box 25046, Mail Stop 415

Denver Federal Center

Lakewood, Colorado 80225 
Metric conversion factors. . . . . . . . . . . . . . . . IV

Abstract ........ . . . . . . . . . . . . . . . 1

Introduction . . . . . . . . . . . . . . . . . . . . 2

Purpose . . . . . . . . . . . . . . . . . . . . . 2

Scope . . . . . . . . . . . . . . . . . . . . 2

Acknowledgments . . . . . . . . . . . . . . . . . 5

Direction of ground-water movement . . . . . . . . . . . . . . . . 5

Effect of waste-disposal activities on ground-water quality. . . . . . 11

Need for further water-quality monitoring. . . . . . . . . . . . 17

Summary. . . . . . . . . . . . . . . . . . . . . . 18

References . . . . . . . . . . . . . . . . . . . . 19

Supplemental information . . . . . . . . . . . . . . . . 21

Description of wells. . . . . . . . . . . . . . . . . . . 22

Records of water levels in wells. . . . . . . . . . . . . 26

Logs of wells drilled by the U.S. Geological Survey . . . . . . . 40

Chemical analyses of sewage sludge and water from the landfill

liquid-waste-disposal trench. . . . . . . . . . . . . 50

Chemical analyses of water from wells . . . . . . . . . . 53

\section{ILLUSTRATIONS}

Plate 1. Map showing geology, location of wells and geologic sections, and water-level contours in alluvium for May 1975 near a sewage-sludge recycling site and a landfill near Denver, Colorado. . . . . . . . In pocket

2. Map showing potentiometric contours for the upper and lower parts of the bedrock formations near a sewage-sludge recycling site and a landfill near Denver, Colorado. . . . . . . . . . . In pocket

Figure 1. Map showing location of study area. . . . . . . . . . . . . 3

2. Diagram showing we11-numbering system . . . . . . . . . 4

3. North-south oriented geologic section . . . . . . . . 6

4. East-west oriented geologic section . . . . . . . . . . . . 7

5. Graph showing magnitude of hydraulic conductivity for different classes of sediment . . . . . . . . . 10

6. Graph showing distribution of mean chloride and dissolvedsolids concentrations in wells perforated in the alluvium 
Table 1. Description of wells . . . . . . . . . . . . . . . . 22

2. Records of water level in wells. . . . . . . . . . . . 26

3. Logs of wells drilled by the U.S. Geological Survey. . . . . 40

4. Chemical analyses of sewage sludge and water from the landfill liquid-waste-disposal trench. . . . . . . . 50

5. Chemical analyses of water from wells. . . . . . . . . 53

6. Recommended limits for dissolved constituents in public water supplies . . . . . . . . . . . . . . 13

7. Average nitrate concentration in selected wells from October 1974 to March 1976 . . . . . . . . . . . . 16

METRIC CONVERSION FACTORS

To convert English units

inches (in)

feet $(f t)$

miles (mi)

feet per day ( $\mathrm{ft} / \mathrm{d}$ )

feet per year

feet per mile

acres

million gallons

gallons per minute (gal/min)

cubic yards

tons,

tons per acre
Muztiply by

25.40

.3048

1.609

.3048

.3048

.1894

$4.047 \times 10^{-3}$

$3.785 \times 10^{3}$

3.785

.7646

.9072

2. 242

To obtain metric units

$$
\begin{aligned}
& \text { millimeters }(\mathrm{mm}) \\
& \text { meters }(\mathrm{m}) \\
& \text { kilometers }(\mathrm{km}) \\
& \text { meters per day }(\mathrm{m} / \mathrm{d}) \\
& \text { meters per year }(\mathrm{m} / \mathrm{yr}) \\
& \text { meters per kilometer }(\mathrm{m} / \mathrm{km}) \\
& \text { square kilometers }\left(\mathrm{km}^{2}\right) \\
& \text { cubic meters }\left(\mathrm{m}^{3}\right) \\
& \text { liters per minute }(\mathrm{L} / \mathrm{min}) \\
& \text { cubic meters }\left(\mathrm{m}^{3}\right) \\
& \text { metric tons }(\mathrm{t}) \\
& \text { metric tons per hectare } \\
& \quad(\mathrm{t} / \mathrm{ha})
\end{aligned}
$$

One microgram per liter $(\mu g / L)$ is approximately equal to one part per billion. One milligram per liter (mg/L) is equal to $1,000 \mu \mathrm{g} / \mathrm{L}$ and is approximately equal to one part per million. 


\title{
RECYCLING SITE AND A LANDFILL NEAR DENVER, COLORADO
}

By S. G. Robson

\begin{abstract}
The Metropolitan Denver Sewage Disposal District and the City and County of Denver operate a sewage-sludge recycling site and a landfill in an area about 15 miles ( 24 kilometers) east of Denver. The assessment of the effects of these facilities on the ground-water system included determining the direction of ground-water movement in the area, evaluating the impact of the wastedisposal activities on the chemical quality of local ground water, and evaluating the need for continued water-quality monitoring.
\end{abstract}

Surficial geology of the area consists of two principal units: (1) Alluvium with a maximum thickness of about 25 feet ( 7.6 meters) deposited along stream channels, and (2) bedrock consisting of undifferentiated Denver and Dawson Formations. Ground water in formations less than 350 feet (110 meters) deep moves to the north, as does surface flow, while ground water in formations between 570 and 1,500 feet (170 and 460 meters) deep moves to the west. Estimates of ground-water velocity were made using assumed values for hydraulic conductivity and porosity, and the observed hydraulic gradient from the study area. Lateral velocities are estimated to be 380 feet (120 meters) per year in alluvium and 27 feet ( 8.2 meters) per year in the upper part of the bedrock formations. Vertical velocity is estimated to be 0.58 foot $(0.18$ meter) per year in the upper part of the bedrock formations.

Potentiometric head decreases with depth in the bedrock formations indicating a potential for downward movement of ground water. However, waterquality analysis and the rate and direction of ground-water movement suggest that ground-water movement in the area is primarily in the lateral rather than the vertical direction.

Five wells perforated in alluvium were found to have markedly degraded water quality. One well was located in the landfill and water that was analyzed was obtained from near the base of the buried refuse, two others were located downgradient and near sewage-sludge burial areas, and the remaining two are located near stagnant surface ponds. Concentrations of nitrate in 
wells downgradient from fields where sludge is plowed into the soil were higher than background concentrations due to the effects of the sludge disposal. No evidence of water-quality degradation was detected in deeper wells perforated in the bedrock formations. Continued water-quality monitoring is needed because of the continuing disposal of wastes. A suggested monitoring program would consist of monitoring wells near the landfill twice a year and monitoring wells near the sludge-disposal areas on an annual basis.

\section{INTRODUCTION}

The Metropolitan Denver Sewage Disposal District operates a 2,000-acre $\left(8-\mathrm{km}^{2}\right)$ sewage-sludge recycling site in Arapahoe County about 15 miles $(24 \mathrm{~km})$ east of Denver, Colo. (fig. 1). The sludge is hauled by truck to the site and either spread on the ground and plowed into the soil or buried in bulk if the weather is inclement. In addition, the City and County of Denver operates a solid and liquid waste landfill on about 250 acres $\left(1.0 \mathrm{~km}^{2}\right)$ adjacent to the sludge-disposal site. Solid waste is trucked to the landfill, dumped, and periodically compacted and covered with earth. Liquid waste is discharged to unlined earth trenches until several million gallons of liquid have accumulated. The trench is then filled with refuse and covered with a layer of earth.

These disposal activities provide a potential source of pollutants which could adversely affect the chemical quality of water in the area. The semiarid climate (14 in or $360 \mathrm{~mm}$ of mean annual precipitation) and the low rolling hills combine to produce minimal runoff in the small ephemeral streams that originate in or near the study area and constitute the surface-drainage network. As a result, the quality of ground water is of primary concern, for ground water is found at shallow depth in some locations and is the only reliable source of water in the area.

\section{Purpose}

The purpose of this study was: (1) To determine the direction of groundwater movement in the alluvial and bedrock aquifers underlying the area, (2) to evaluate the effects of the waste-disposal activities on the chemical quality of the ground water, and (3) to determine the need for future water-quality monitoring in the area.

\section{Scope}

The scarcity of existing wells in the study area required that additional observation wells be drilled. As shown on plate 1 and in table 1 (at back of report), 41 observation wells were installed at depths ranging from 4 to 248 feet $(1$ to $76 \mathrm{~m})$. The tables in this report present pertinent data for thest wells in addition to the 17 previously existing wells in the study area. The wel1-numbering system used in this study indicates the location of the well by quadrant, township, range, section, and position within the section, as illustrated in figure 2 . 


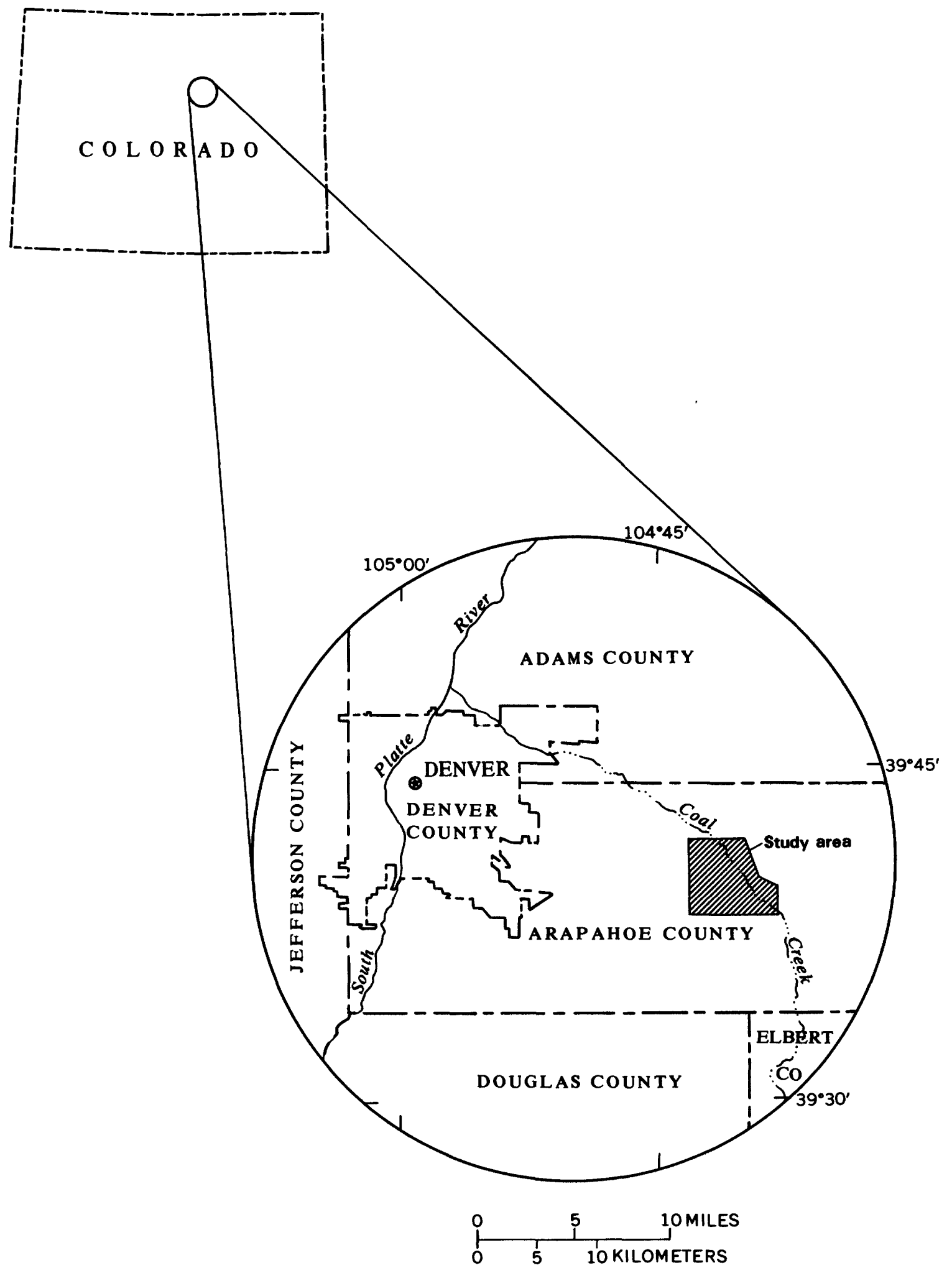

Figure 1.-Location of study area. 


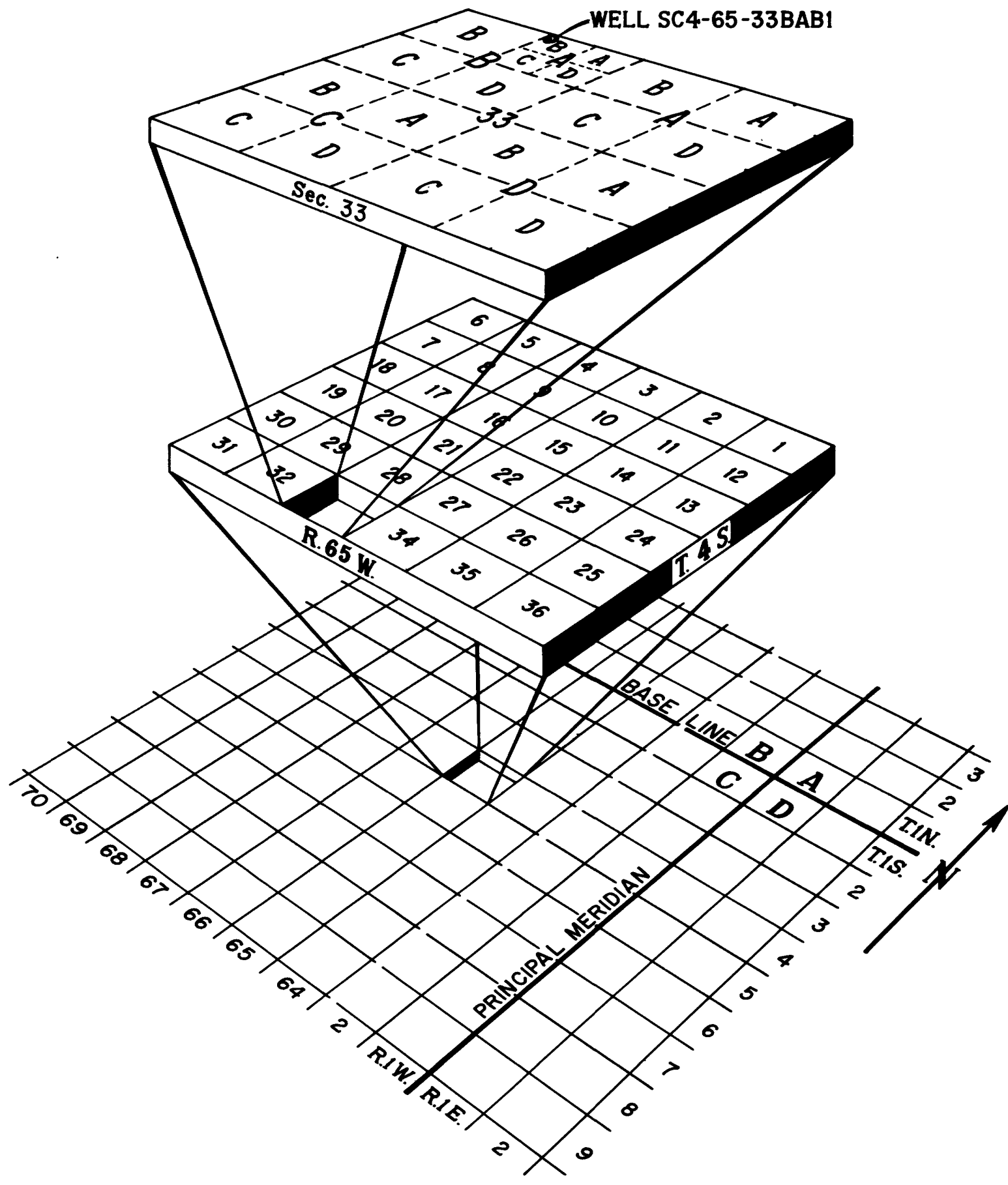

Figure 2.-Well-numbering system. 


\section{Acknowledgments}

The study was made in cooperation with the Metropolitan Denver Sewage Disposal District and the Colorado Geological Survey. Much of the laboratory analytical work was done by the Metropolitan Denver Sewage Disposal District under the direction of laboratory supervisor Car1 Calkins. Their assistance is gratefully acknowledged.

\section{DIRECTION OF GROUND-WATER MOVEMENT}

The determination of the direction of ground-water movement requires an understanding of the geology of the area before water-level measurements in wells can be properly interpreted to show the direction of movement. The direction of movement in conjunction with estimates of the rate of ground-water movement provides a means of evaluating the potential effects of movement of degraded ground water.

The surficial geology of the area consists of two principal units: Alluvium consisting of unconsolidated, poor- to moderately we11-sorted clay, silt, sand, and gravel of Pleistocene and Holocene age with a maximum thickness of about 25 feet $(7.6 \mathrm{~m})$; and (2) the undifferentiated Denver and Dawson Formations consisting of brown, dusky-yellow, and blue-gray mudstone with thin, lenticular beds of 1ignite and gray sandstone. The Denver and Dawson Formations are of Late Cretaceous and Paleocene age and extend from land surface to a depth of 1,570 feet $(478 \mathrm{~m}$ ) in well SC 5-65- 5BDA (McConaghy and others, 1964). The mudstone units are dense, moderately consolidated, and show little evidence of joints or fracture cleavage. Lignite beds encountered during drilling of observation wells did not yield measurable quantities of water to the wells. The sandstone beds are the only units capable of yielding measurable quantities of water to the observation wells in the consolidated formations. The geologic sections (figs. 3 and 4) show the relation of the alluvium and the sandstone and 1ignite in the upper part of the bedrock formations. The bedrock formations dip to the northwest at about 1 degree near the west edge of the study area and are relatively flat-lying in the remainder of the area.

The yield of most of the observation wells perforated in the alluvium was about 0.1 gallon per minute $(0.4 \mathrm{~L} / \mathrm{min})$ with a few wells yielding as much as 10 gallons per minute $(38 \mathrm{~L} / \mathrm{min})$. All the wells having higher yields are 1ocated along Coal Creek, an area where field examination of drill cuttings indicated the alluvium to be coarser, better sorted, and, therefore, more permeable. The wells with lower yields are due to the small saturated thickness, fine grain size, and poor sorting of most of the alluvial materials in the remainder of the area. Yields from about 200 feet $(60 \mathrm{~m})$ of hole drilled in the bedrock normally did not exceed 5 gallons per minute $(19 \mathrm{~L} / \mathrm{min})$, although the sandstone encountered at a depth of 50 feet $(15 \mathrm{~m})$ in well SC 5-65- 6CAC2 yielded about 20 gallons per minute $(80 \mathrm{~L} / \mathrm{min})$ during drilling.

Water-table contours for the alluvial aquifer (p1.1) indicate that ground water moves down the alluvial valleys in a direction controlled by the geology and the orientation of the valley. The general direction of movement is to the north or northwest. 


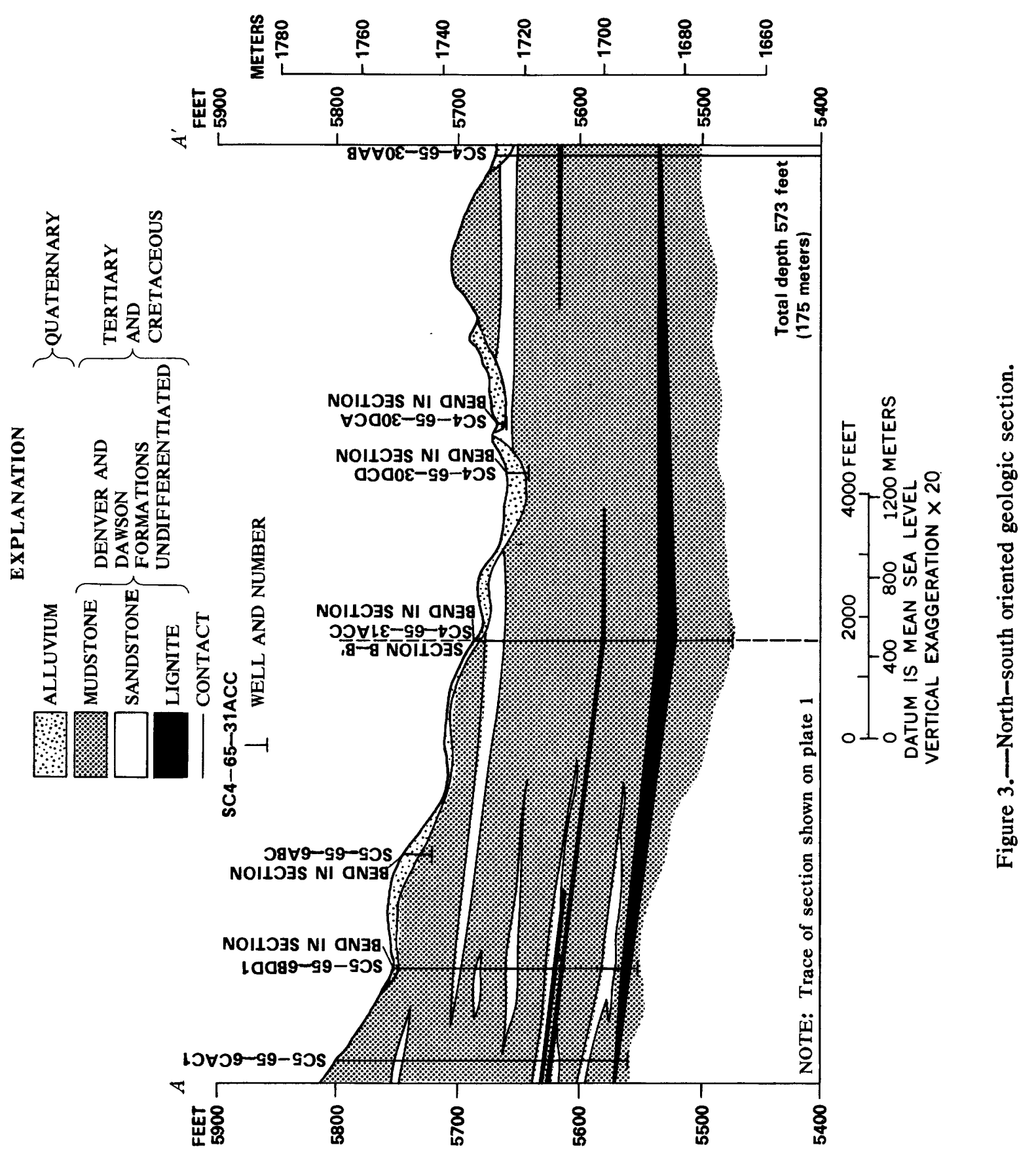




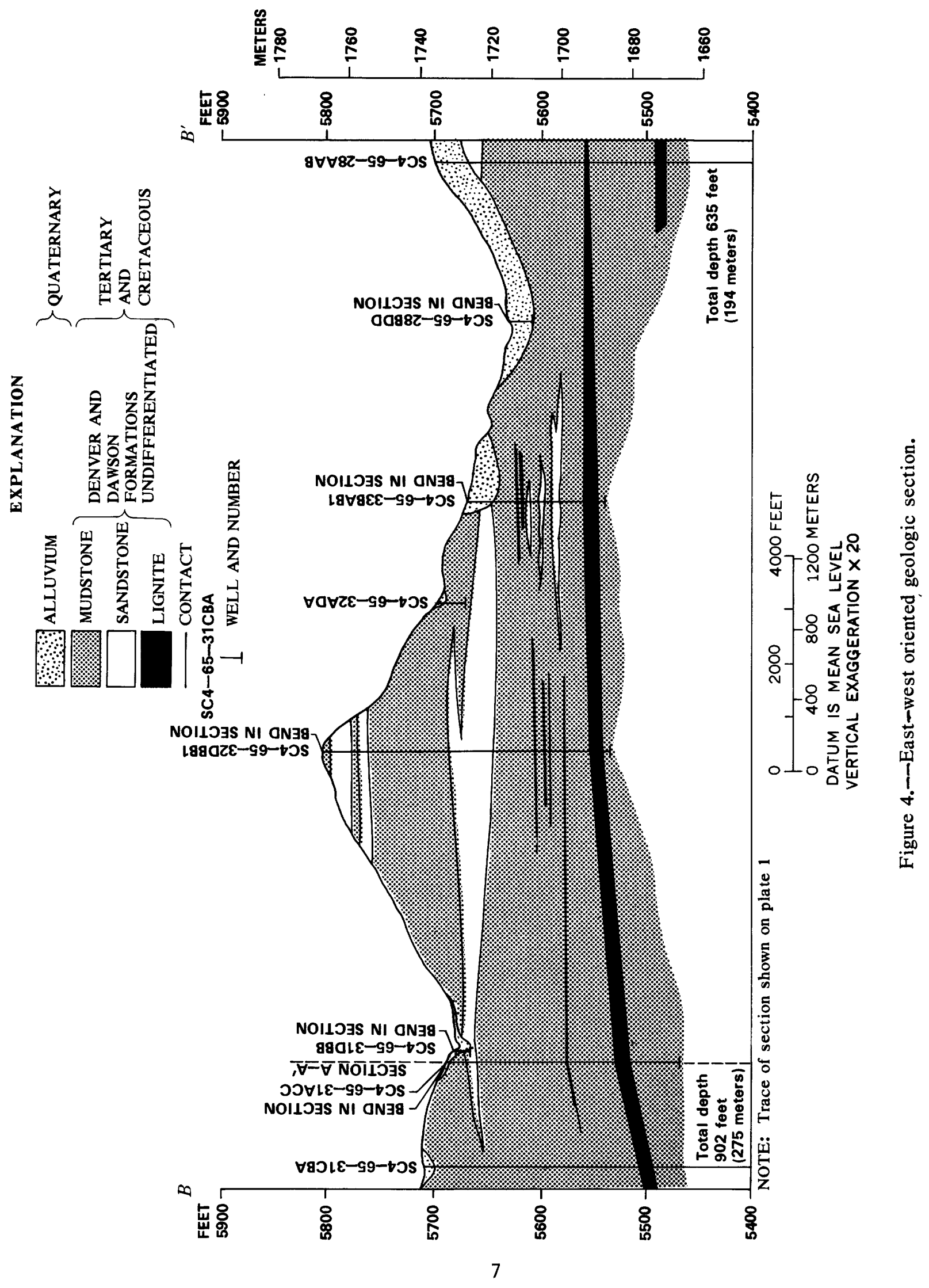


When water-level measurements in wells tapping confined aquifers are used in contouring, the resulting map is termed a potentiometric-contour map (p1.2). The solid potentiometric contours on plate 2 represent the elevation at which water stands in wells whose depths range from 100 to 350 feet (30 to $110 \mathrm{~m}$ ). Wells of this depth are perforated in the upper part of the undifferentiated bedrock formations. A comparison of plates 1 and 2 indicates that the potentiometric surface in the upper part of the bedrock is generally 10 to 40 feet ( 3 to $10 \mathrm{~m}$ ) below the water table in the alluvium. The potential thus exists for ground-water movement from the alluvium into the upper part of the bedrock. The general direction of ground-water movement in the upper part of the bedrock, as in the alluvium, is to the north.

Water-level measurements in wells perforated in the alluvium or the upper part of the bedrock indicate that only minor water-level changes have occurred in these zones from 1974 to 1976. However, 1974 water-level measurements in frequently pumped domestic wells (wells SC 4-65-28AAB and SC 4-65-30AAB, for example) perforated in the lower part of the bedrock formations are as much as 100 feet $(30 \mathrm{~m})$ below measurements made at the time the wells were drilled in 1971. In order to avoid considering the effects of localized water-level declines around pumping wells, the potentiometric-contour map for the lower part of the bedrock is based on measurements made at the time each well was drilled. The dashed potentiometric contours on plate 2 are based on water-level measurements in five wells ranging in depth from 570 to 1,570 feet (170 to $480 \mathrm{~m}$ ) and represent the potentiometric surface in the lower part of the bedrock formations. In this depth interval the direction of ground-water movement generally is to the west. The same direction of local movement occurs in aquifers at depths between 1,800 and 2,000 feet (550 and $610 \mathrm{~m}$ ) as found by Romero (1976).

An unusual condition thus exists, in which ground water in the alluvium and the upper part of the bedrock formations moves to the north while ground water in the lower part of the bedrock formation moves to the west. This suggests that the aquifers in the two depth intervals do not have good hydraulic connection. The divergence in the direction of ground-water movement coexists with large differences in head with depth in the formations. Near well SC 565- 6CDA, for example, the elevation of the water table in the alluvium is about 40 feet $(12 \mathrm{~m})$ higher than the potentiometric surface in the upper part of the bedrock, and about 250 feet $(76 \mathrm{~m})$ higher than the potentiometric surface in the lower part of the bedrock. By contrast, near well SC 4-65-28BDD the total difference between the elevation of the water table in the alluvium and the potentiometric surface in the lower part of the bedrock is about 60 feet $(18 \mathrm{~m})$. The vertical differences in head create a potential for downward movement of ground water in addition to the potential for lateral movement indicated by the potentiometric-contour maps.

Both the rate and direction of ground-water movement are of prime concern in any study of ground-water contamination. In order to calculate the rate of ground-water movement, it is necessary to have data describing the ability of the saturated sediments to transmit water (hydraulic conductivity) and the volume of pore space in the sediments (porosity). These characteristics of the sediment are used in conjunction with the hydraulic gradient (slope of the 
water table or potentiometric surface) to calculate the ground-water velocity by use of the equation:

$$
V=\frac{K I}{\phi} \times 365
$$

where $V=$ ground-water velocity, in feet per year, $K=$ hydraulic conductivity, in feet per day,

$I=$ hydraulic gradient (dimensionless, feet per feet), and

$\phi=$ porosity (dimensionless).

Data on the hydrologic properties of the sediments in the study area are not available; consequently, the actual ground-water velocities in the area cannot be calculated. However, data describing the hydrologic character of the alluvium in sec. 28 , T. 4 S., R. 67 W., and secs. 20 and 23, T. 5 S., R. $66 \mathrm{~W}$., are available, as are data for the upper part of the bedrock formations in sec. 18, T. 4. S., R. 65 W. and sec. 24, T. 6 S., R. 66 W. (McConaghy and others, 1964). If these data are used in conjunction with the hydraulic gradients in the study area, the rate of ground-water movement can be calculated for sediments of similar hydraulic conductivity and porosity in the study area. However, it is unknown whether or not the data describing the hydrologic character of the alluvium and bedrock outside the study area reflect the hydrologic character of the units in the study area. As a result of these uncertainties, the velocities calculated using these data are considered to be general indications of the magnitude of ground-water velocities and not actual. velocities in the study area.

The hydraulic conductivity of 10 samples from alluvium 2 to 9 miles ( 3 to $14 \mathrm{~km})$ from the study area ranged from $8 \times 10^{-3}$ to 130 feet per day $\left(2 \times 10^{-3}\right.$ to $40 \mathrm{~m} / \mathrm{d}$ ) with a mean of 32 feet per day $(10 \mathrm{~m} / \mathrm{d})$ ( $\mathrm{fig} .5)$. The mean porosity was 34.2 percent. When the hydraulic gradient $(0.011)$ in the alluvium in the study area is used, the resulting lateral ground-water velocities range from $9.4 \times 10^{-2}$ to 1,500 feet per year $\left(2.9 \times 10^{-2}\right.$ to $\left.460 \mathrm{~m} / \mathrm{yr}\right)$ with a mean of 380 feet per year $(120 \mathrm{~m} / \mathrm{yr})$. The ground-water velocities in the alluvium of Coal Creek are probably much higher than those in the other alluvial areas as are the yields of wells near Coal Creek. The hydraulic conductivity of six samples taken from the upper part of the bedrock formations 1 to 8 miles ( 2 to $13 \mathrm{~km})$ from the study area range from $9.4 \times 10^{-5}$ to 8.7 feet per day $\left(2.9 \times 10^{-5}\right.$ to $2.7 \mathrm{~m} / \mathrm{d}$ ) with a mean of 2.8 feet per day $(0.85 \mathrm{~m} / \mathrm{d})$. The mean porosity was 41.4 percent. Using the hydraulic gradient in the upper part of the bedrock in the study area $(0.011)$, the lateral ground-water velocities were calculated to range from $9.1 \times 10^{-4}$ to 84 feet per year $\left(2.8 \times 10^{-4}\right.$ to $\left.26 \mathrm{~m} / \mathrm{yr}\right)$ with a mean of 27 feet per year $(8.2 \mathrm{~m} / \mathrm{yr})$.

The rate of vertical ground-water movement is also of concern because of the head change with depth in the formations. The rate of vertical movement in saturated materials is primarily controlled by the stratum having the lowest vertical hydraulic conductivity and greatest thickness. If the siltstone in the upper part of the bedrock formations near the landfill is the stratum of lowest vertical hydraulic conductivity, the combined thickness of the stratum at well SC 5-65- 6DBC, for example, may be used to calculate the vertical hydraulic gradient. Data in tables 1, 2, and 3 (at back of report) indicate that 


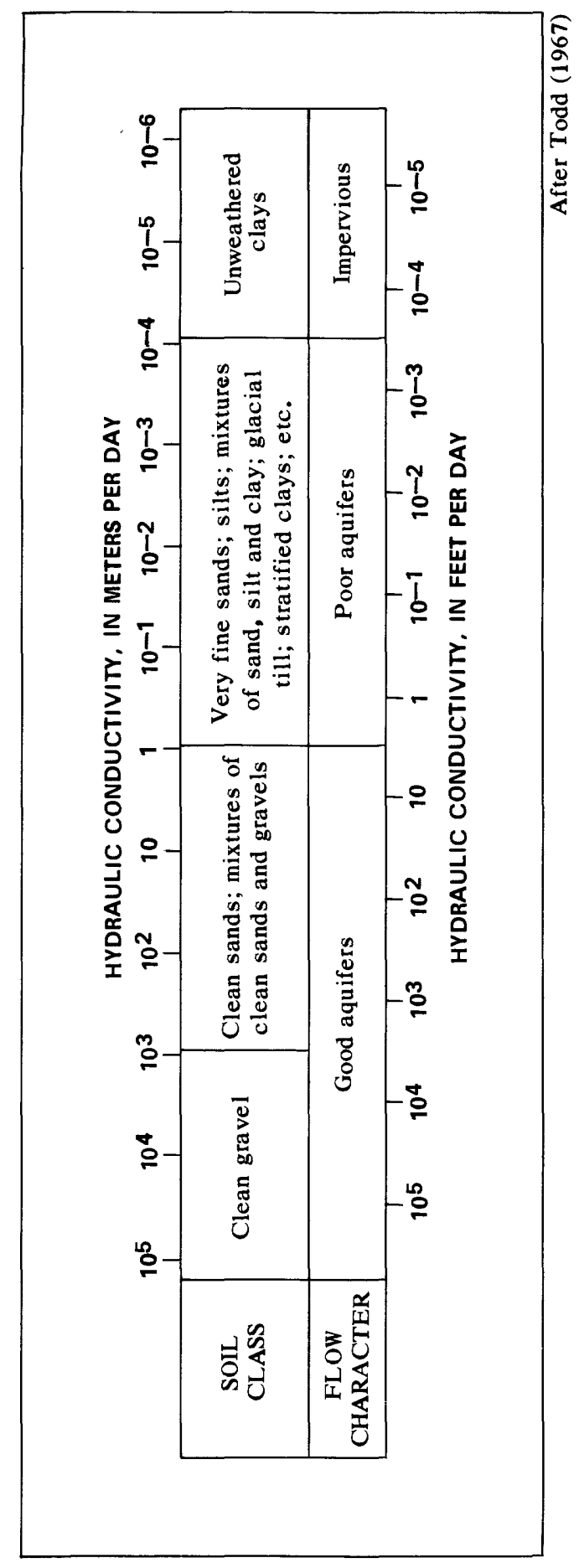

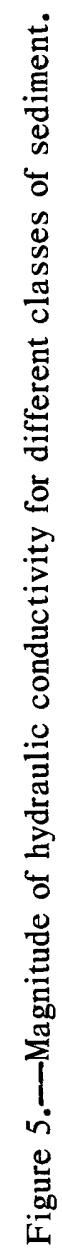


a head difference of 6.5 feet $(2.0 \mathrm{~m})$ exists across a zone about 160 feet $(50 \mathrm{~m})$ thick consisting primarily of siltstone. As in the previous calculations, the hydraulic conductivity and porosity of the bedrock in the study area are not known. If hydraulic properties comparable to those used for the lateral velocity calculations are considered, the vertical hydraulic conductivity of the siltstone likely would be between the average and minimum horizontal hydraulic conductivity for the upper part of the bedrock. A vertical velocity of 0.58 foot per year $(0.18 \mathrm{~m} / \mathrm{yr})$ is calculated by using a vertical hydraulic conductivity midway between the mean and minimum lateral hydraulic conductivity $\left(1.6 \times 10^{-2} \mathrm{ft} / \mathrm{d}\right.$ or $\left.4.9 \times 10^{-3} \mathrm{~m} / \mathrm{d}\right)$ and a porosity of 41.4 percent. However, if the vertical hydraulic conductivity was equal to the minimum lateral hydraulic conductivity $\left(9.4 \times 10^{-5} \mathrm{ft} / \mathrm{d}\right.$ or $\left.2.9 \times 10^{-5} \mathrm{~m} / \mathrm{d}\right)$, the vertical velocity would equal $3.4 \times 10^{-3}$ foot per year $\left(1.0 \times 10^{-3} \mathrm{~m} / \mathrm{yr}\right)$.

If the average velocities are assumed to be representative of the general ground-water velocities to occur in the study area, the movement and possible effects of sources of ground-water degradation can be evaluated. If water in the upper part of the bedrock formations were degraded near the landfill, for example, it would require about 500 years for the degraded water to move 2.5 miles $(4 \mathrm{~km})$ from the landfill to the nearest domestic well (SC 4-65-30AAB). During this movement, the degraded water would be diluted by recharge of nondegraded water from the alluvium and would be further diluted by mixing with nondegraded water in the upper part of the bedrock formations. Unless the landfill becomes a long-term source of large quantities of leachate containing high concentrations of degrading constituents, it is unlikely that the degradation would have a significant effect on the ground-water quality at well SC 4-65-30AAB in the foreseeable future.

The ground-water-flow path in the alluvium from the sludge disposal area in sec. 32 , T. 4. S., R. $65 \mathrm{~W}$., to domestic well SC 4-65-28BBC is about 1.6 miles $(2.6 \mathrm{~km})$. If the alluvium has an average hydraulic conductivity and porosity of 32 feet per day $(10 \mathrm{~m} / \mathrm{d})$ and 34.2 percent, it would require about 30 years for ground water to move from the east edge of sec. 32 , T. 4, S., R. 65 W., to well SC 4-65-28BBC with the local hydraulic gradient of $7.6 \times 10^{-3}$. Unless the sludge-disposal area becomes a long-term source of a large quantity of leachate containing high concentrations of degrading constituents, the impact on the water quality at well SC 4-65-28BBC would appear to be small.

\section{EFFECT OF WASTE-DISPOSAL ACTIVITIES ON GROUND-WATER QUALITY}

Land disposal of sludge began in the study area in 1969 and by 1976 application rates ranged from about 60 to 210 dry tons per acre (130 to 470 $\mathrm{t} / \mathrm{ha}$ ) in the areas where the sludge is plowed into the soil. About 34,000 dry tons $(31,000 \mathrm{t})$ of sludge were buried in the burial site in sec. 9, T. $5 \mathrm{~S}$. , R. 65 W., between 1969 and 1970 (p1. 1). The current burial site in sec. 4, T. 5 S., R. $65 \mathrm{~W}$., has received 3,000 to 4,000 tons $(3,000$ to 4,000 $t$ ) of sludge since 1973 (Don Grasmick, Metropolitan Denver Sewage Disposal District, written and oral commun., 1976). The chemical nature of the sludge varies considerably depending on the source of the sludge within the Metropolitan Denver Sewage Disposal District and the type of chemicals used to aid precip- 
itation. The three principal types of sludge are not segregated in the disposal area; as a result, the quality of the composite sludge is probably a combination of the three analyses shown in table 4 (at back of report). The composite sludge is high in calcium, total Kjeldahl nitrogen, ammonia, phosphorus, and the trace metals, cadmium, chromium, copper, lead, nickel, and zinc.

The landfill began operation in 1966 and by about 1972 liquid-waste trenches capable of holding in excess of 1 million gallons $\left(4,000 \mathrm{~m}^{3}\right)$ were being used (Jerry Bone11o, City and County of Denver, oral commun., 1976). By 1975, between 1 and 2 million cubic yards ( 1 to 2 million $\mathrm{m}^{3}$ ) of compacted refuse had been buried at the site. The chemical quality of leachate moving through the buried refuse can be determined only by sampling a well drilled into the refuse, for no surface discharge of leachate occurs. Wel1 SC 5-65$6 \mathrm{CDA}$, perforated at the base of the fill materials, provides the best available indication of the leachate quality (see table 5 at back of report). Water from this well is particularly high in chloride, dissolved solids, sodium, iron, and manganese.

Analyses of water samples taken from a liquid waste trench at the landfill are shown in table 4 (at back of report) and indicate that the water contains high concentrations of all the determined constituents with very high concentrations of sodium, potassium, and phenols.

Because ground-water quality was not monitored in the area prior to the beginning of disposal activities, the background water quality can be inferred only from the water quality in nearby wells in areas not likely to have been affected by the disposal activities. Four wells, by virtue of their location, are thought to be representative of the background water quality in the alluvium. Data from wells SC 4-65-30BDD, SC 4-65-31DDC, SC 5-65-3ABB, and SC 565- 9DDA indicate that, with the exception of iron and manganese concentrations, the background water quality meets the U.S. Environmental Protection Agency's recommended drinking-water standards (1973) (table 6) and has dissolved-solids, sodium, chloride, and nitrate (as $\mathrm{NO}_{3}$ ) concentrations of about $550,50,11$, and $0.1 \mathrm{mg} / \mathrm{L}$, respectively. Wells SC 4-65-30BDA and SC 5-65$8 \mathrm{BCB}$ are thought to be representative of background water quality in the upper part of the bedrock formations. This water meets the U.S. Environmental Protection Agency's recommended drinking-water standards (1973) and has dissolvedsolids, sodium, chloride, and nitrate (as $\mathrm{NO}_{3}$ ) concentrations of about 350 , 100,50 , and $0.1 \mathrm{mg} / \mathrm{L}$, respectively. We11 SC 5-65- 5BDA is perforated in the lower part of the bedrock formations and is assumed to be representative of the background water quality in this zone. The water meets the U.S. Environmental Protection Agency's recommended drinking-water standards (1973) and has dissolved-solids, sodium, chloride, and nitrate concentrations of about 200 , 100,5 , and $0.2 \mathrm{mg} / \mathrm{L}$, respectively. The ground water in both the alluvium and bedrock is of sodium bicarbonate type. The occurrence of water of lower dissolved-solids concentration at greater depth in the geologic section suggests that the primary source of the water at depth in the study area is not the downward movement of water from the alluvium but lateral movement from areas to the south or east. The water-quality data substantiate the data on the general direction and rates of ground-water movement which indicate that the movement in the area is primarily in the lateral rather than the vertical direction. 
Table 6.--Recommended limits for dissolved constituents in public water supplies

[Data from U.S. Environmental Protection Agency, 1973]

\begin{tabular}{|c|c|c|c|}
\hline \multicolumn{2}{|c|}{$\begin{array}{l}\text { Constituents that may be } \\
\text { toxic in high concentrations }\end{array}$} & \multicolumn{2}{|c|}{$\begin{array}{l}\text { Constituents that may affect } \\
\text { potability in high concentrations }\end{array}$} \\
\hline Constituent & $\begin{array}{l}\text { Recommended } \\
\text { limit, in } \\
\text { milligrams } \\
\text { per liter }\end{array}$ & Constituent & $\begin{array}{l}\text { Recommended } \\
\text { limit, in } \\
\text { milligrams } \\
\text { per liter }\end{array}$ \\
\hline Arsenic- & 0.1 & Ammonia- & 0.5 \\
\hline Barium- & 1.0 & Chloride- & 250.0 \\
\hline Cadmium-- & .01 & Copper-1- & 1.0 \\
\hline Chromium- & .05 & Iron- & .3 \\
\hline Cyanide-_- & .2 & Manganese- & .05 \\
\hline Lead- & .05 & Pheno1s - & .001 \\
\hline Mercury- & .002 & Sulfate- & 250.0 \\
\hline Nitrate (as N) & 10.0 & Zinc- & 5.0 \\
\hline Nitrite (as N)- & 1.0 & & \\
\hline \multicolumn{4}{|l|}{ Pesticides: } \\
\hline Aldrin- & .001 & & \\
\hline Chlordane- & .003 & & \\
\hline DDT- & .05 & & \\
\hline Dieldrin-_- & .001 & & \\
\hline Endrin- & .0005 & & \\
\hline Heptachlor & .0001 & & \\
\hline Heptachlor Epoxide- & .0001 & & \\
\hline Lindane- & .005 & & \\
\hline Methoxychlor-_-_- & 1.0 & & \\
\hline Toxaphene- & .005 & & \\
\hline Selenium & .01 & & \\
\hline
\end{tabular}

From October 1974 to March 1976, about 200 water-quality samples were collected from wells perforated in the alluvium (tables 1 and 5 at back of report). A review of these data indicates that dissolved solids, sodium, chloride, and nitrate are better indicators of ground-water-quality degradation in the area than are the other determined constituents. Although concentrations of phenols and trace metals are known to be high in some of the waste, these constituents were not consistently detected in elevated concentrations near disposal areas. The high clay content of the sediments in the study area may be largely responsible for this, for these dissolved constituents are readily bound to clay minerals and their movement is thus inhibited.

Chloride and dissolved solids, by contrast, are highly mobile in the ground-water environment and are commonly excellent indicators of groundwater-quality degradation (Hughes and Robson, 1973; Palmquist and Sendlein, 
1975; and Zanon, 1971). Because the temporal change in the concentration of dissolved constituents was not large (with the exception of we11 SC 5-65$6 \mathrm{CDA})$, mean concentrations calculated for each wel1 over the 18-month sampling period are representative of the water quality commonly found in each well. By plotting the mean chloride and dissolved-solids concentrations for each well during the sampling period, a pattern of points is produced which has significance when the location of wells containing degraded and nondegraded ground water is known (fig. 6). It can be seen in figure 6 that the four wells representing background water quality in the alluvium (we11s SC 4-6530BDD, SC 4-65-31DDC, SC 5-65- 3ABB, and SC 5-65- 9DDA) plot in the lower part of the graph while the well with degraded water in the landfil1 (we11 SC 5-656CDA) plots in the extreme upper part of the graph. If the pattern of points is divided into three arbitrary regions, it can be seen in table 5 (at back of report) that wells in region 1 have water-quality characteristics similar to the background water quality in the area and are, therefore, least likely to have been affected by degradation from waste-disposal activities. We11s in region 3 have water-quality characteristics more similar to that of degraded ground water ( $t a b l e ~ 5$ at back of report) and are likely to have been affected by a source of degradation. Wells in region 2 yield water of intermediate quality with no clear indication of degradation.

There are readily apparent sources of ground-water degradation near each of the five wells in region 3. Well SC 5-65- 6CDA is located in the 1andfill refuse. Water from this well exceeds the U.S. Environmental Protection Agency's recommended drinking-water standards (1973) (tab1e 6) for chloride, su1fate, iron, manganese, and lead. Wells SC 5-65- 4DBC and SC 5-65-9ACD are located immediately downslope of two large areas used to bury sewage sludge (p1. 1). Leachate from the sludge could be affecting the ground-water quality near the two wells. The water quality is similar in both we11s with nit rate, chloride, ammonia, and magnesium concentrations much higher than background concentrations, and sulfate and manganese concentrations exceeding U.S. Environmental Protection Agency's recommended drinking-water standards (1973). We11s SC 565 4CAC and SC 46533CBC are equipped with windmills and are used to supply water for cattle. The overflow from the stock tanks forms stagnant manure-contaminated ponds which are thought to be the source of degradation in the nearby shallow wells. The water quality in these two wells is similar, both having much higher concentrations of nitrate, chloride, sulfate, and zinc than found in background samples, and sulfate in excess of the U.S. Environmental Protection Agency's recommended drinking-water standards (1973). A11 but two of the wells used to monitor water quality in the alluvium, near areas where sludge is plowed into the soil, plot in region 1 of figure 6 , which suggests that none of the wells are strongly affected by the movement of water of degraded quality.

We11s in which the average nitrate concentration exceeded $0.3 \mathrm{mg} / \mathrm{L}$ (as $\mathrm{NO}_{3}$ ) during the sampling period are shown in table 7. The highest nitrate concentrations occur in wells downgradient from the two sludge-burial areas, near the landfill and near the stock-watering tanks, further indicating that ground-water-quality degradation is occurring in these areas. Nitrate concentrations ranging from 0.4 to $13 \mathrm{mg} / \mathrm{L}$ (as $\mathrm{NO}_{3}$ ) also occur in wells located downgradient from areas where sludge is plowed into the soil. These concen- 


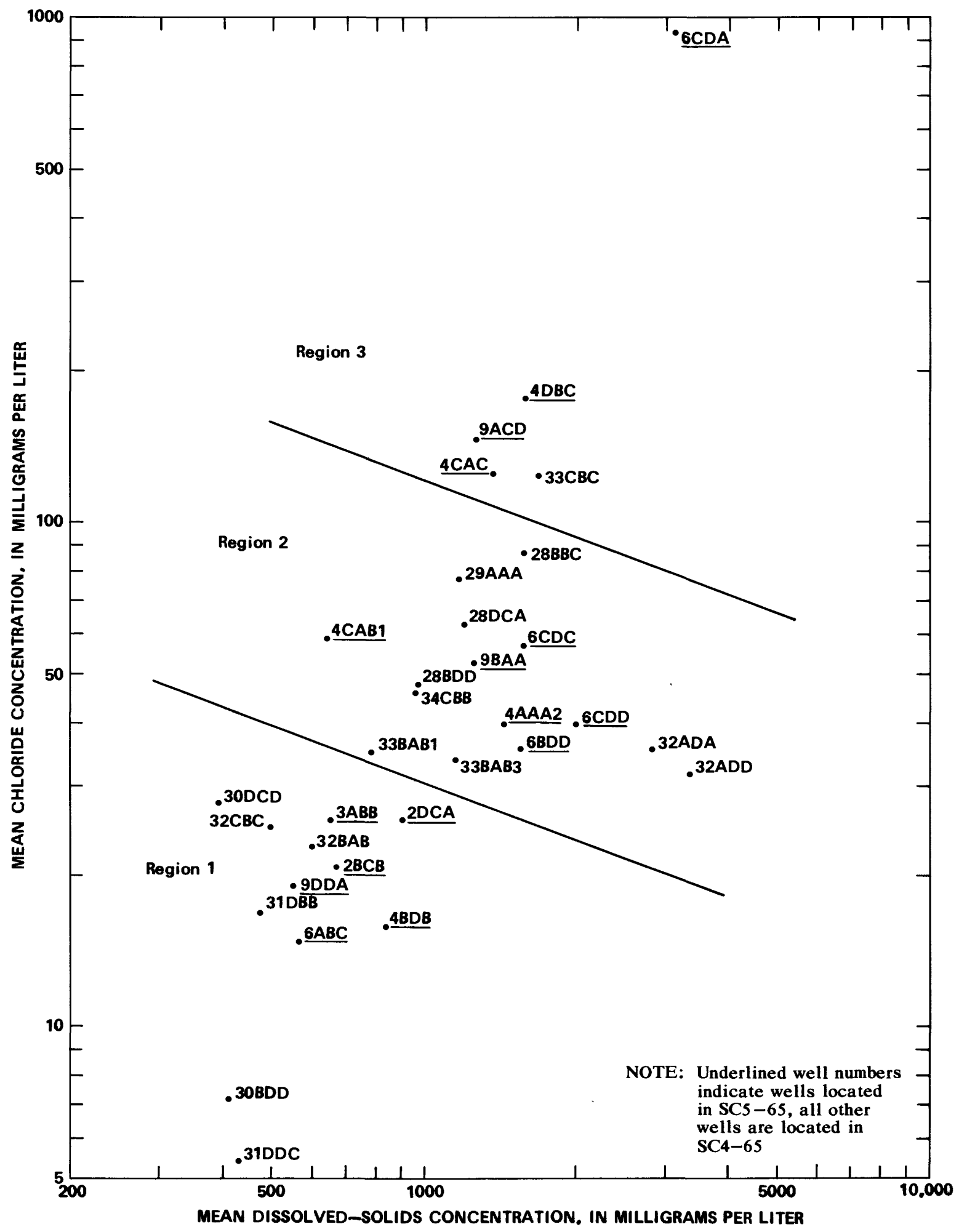

Figure 6.--Distribution of mean chloride and dissolved-solids concentrations in wells perforated in the alluvium. 
Table 7.--Average nitrate concentration in selected wells from October 1974 to March 1976

We11 number

Average nitrate concentration, as $\mathrm{NO}_{3}$, in milligrams per liter

\begin{tabular}{|c|c|}
\hline 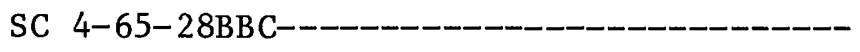 & 4.8 \\
\hline 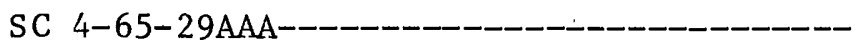 & 2.1 \\
\hline 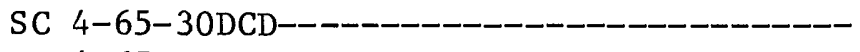 & 2.4 \\
\hline 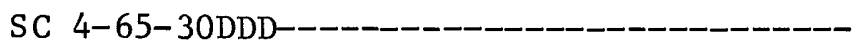 & 6.2 \\
\hline 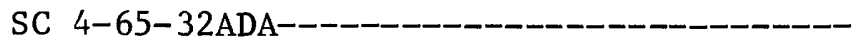 & 13 \\
\hline 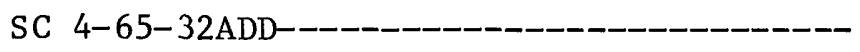 & 3.4 \\
\hline 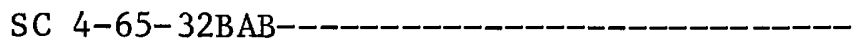 & 9.6 \\
\hline 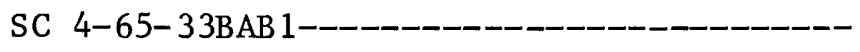 & 4.0 \\
\hline 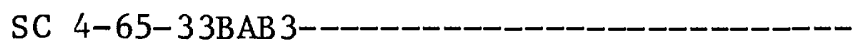 & 1.5 \\
\hline 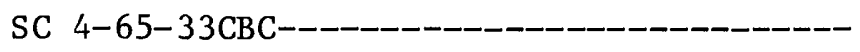 & 21 \\
\hline 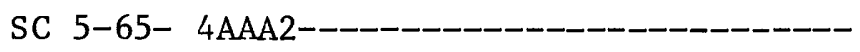 & .42 \\
\hline SC 5-65- 4CAC----ー--ー-ー-ー- & 11 \\
\hline 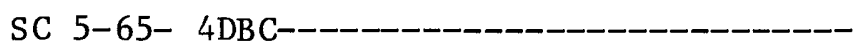 & 30 \\
\hline 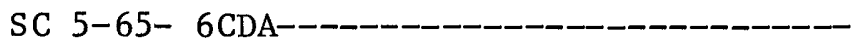 & .50 \\
\hline SC $5-65-6 \mathrm{CDC}--$ & .49 \\
\hline 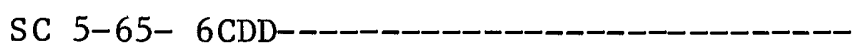 & 15 \\
\hline 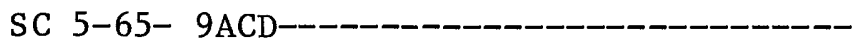 & 24 \\
\hline
\end{tabular}

trations are above the $0.1-\mathrm{mg} / \mathrm{L}$ background concentration in the alluvium and are probably the result of the sludge-disposal practices in the area. The higher-than-background nitrate concentrations in water from we11s SC 4-65$28 \mathrm{BBC}$ and SC 4-65-29AAA are thought to be derived from agricultural sources near the wells.

Ground-water samples taken from 24 wells (table 5 at back of report) in February 1975 were analyzed for fecal coliform and fecal-streptococci bacteria. None of the sampled wells had significant fecal coliform concentrations; however, fecal-streptococci concentrations ranging from 3 to 177 colonies per 100 milliliters of sample were found in 11 widely scattered wells. When these wells were resampled in April 1976, the analysis indicated negligible fecalstreptococci concentrations. In addition to samples for bacterial determinations, eight wells were sampled for insecticides and industrial compounds and five wells were sampled for phenolic compounds. Polychlorinated-biphenols (PCB) were detected in wells SC 5-65- 4AAA and SC 5-65- 4DBC; the concentration was $0.2 \mu \mathrm{g} / \mathrm{L}$. Phenolic compounds were detected in we1l SC 5-65- 6BDD; the concentration was $0.022 \mathrm{mg} / \mathrm{L}$.

Between June 1975 and March 1976, about 60 water-quality samples were taken from 12 wells perforated in the upper part of the bedrock formation 
(table 5 at back of report). During construction of all but three of these we11s (SC44-65-30BDA, SC 5-65- 6BDD2, and SC 5-65- 9BCB), concrete grout used to seal the casing in the well bore inadvertently invaded the water-bearing materials and has had a pronounced effect on the chemical composition of water pumped from the wells. As a result, the $\mathrm{pH}$, dissolved-solids, hardness, calcium, carbonate, bicarbonate, hydroxide, and sulfate determinations for the affected wells are not representative of the water quality in the ground-water system. When the analyses for the wells unaffected by grout invasion are examined in conjunction with the unaffected parts of the analyses from groutinvaded wells, no clear pattern of water-quality degradation can be discerned. Although chloride concentrations are higher than background values in wells SC 4-65-32DBB2 and SC 4-65-32DBB3, as are tota1-Kjeldah1 nitrogen concentrations in wells SC 5-65- 4CAB2 and SC 5-65- 6CAC2 (table 5 at back of report), other dissolved constituents in these wells do not have markedly high concentrations. In addition, no insecticides or industrial compounds were detected in samples from wells SC 4-65-30BDA and SC 5-65- 6CAC1. If ground-water qua1ity in the upper part of the bedrock is being affected by the waste-disposal activities in the area, the resulting changes in water quality are not discernible at this time (1976).

Water-quality analyses are available for three wells (SC 4-65-28AAB, SC 4-65-30AAB, and SC 5-65- 5BDA) perforated in the lower part of the bedrock formations. The water quality in wells SC 4-65-28AAB and $S C$ 4-65-30AAB is similar to the background quality as represented by analysis from wel1 SC 565- 5BDA. The data indicate that the wells in the lower part of bedrock formations show no effect of the waste-disposal activities in the area.

\section{NEED FOR FURTHER WATER-QUALITY MONITORING}

The basic purpose of a water-quality monitoring program in this area would be to record the effect of the waste-disposal activities on the water quality in order to help assure that any adverse effects do not exceed acceptable limits. As of 1976, both the landfill and the sludge-burial areas are sources of only minor ground-water-quality degradation. However, the volume of leachate produced by these sources will likely increase in the future, as more material is deposited and the surface areas of the sites expand. Increased volumes of leachate could produce more rapid and widespread deterioration in ground-water quality and increase the need for water-quality monitoring. Because of the slow rates of ground-water movement to be expected in this area, monitoring would require only infrequent sampling of ground-water quality.

The landfill and the associated liquid-disposal trenches are of firstorder concern in a water-quality-monitoring program because of the large volume of material handled and the lack of control over the type of materials that may be dumped. It is suggested that ground-water-quality monitoring near the landfill consist of sampling all the wells in sec. 6, T. 5 S., R. 65 W., twice a year and analyzing the samples for dissolved solids, sodium, chloride, nitrate, trace metals, and phenolic compounds. Although surface runoff from part of the landfill is presently intercepted by holding ponds, no means exist 
to control the runoff from the entire landfill. Under present conditions, a spill resulting from the failure of a liquid-disposal trench could result in a slug of contaminated liquid which would enter the surface-drainage network and could contaminate riparian land and ground water for many miles downstream. If an earthen dam were constructed near well SC 5-65- 6ABC, surface runoff or accidental spills originating anywhere in the landfill could be contained and the resulting pool and local ground water could be monitored, if a potential for ground-water degradation was found to exist.

Less frequent water-quality monitoring would be needed near sludge-disposal areas because of the lesser potential for ground-water degradation from these areas. Sampling the wells on an annual basis in the spring of each year is suggested to monitor the movement of degraded water. The suggested monitoring program could be achieved by sampling all the wells near the sludgeburial areas and wells SC 4-65-30DCD, SC 4-65-31DBB, SC 4-65-31CBA, SC 4-6532ADA, SC 4-65-32BAB, SC 4-65-33BAB1, SC 4-65-33CBC, SC 5-65- 4AAA2, and SC 565- 6ABC near the plowed disposal fields. Analysis would include ammonia, chloride, zinc, magnesium, sodium, and dissolved solids. Monitoring near the sludge-disposal areas and the landfill would be needed for an indefinite period of time beyond the end of the disposal activities to assure that further leaching of the buried material would not contribute to the degradation of the ground water.

\section{SUMMARY}

As a result of this study it has been determined that the direction of ground-water movement in the alluvium and upper part of the bedrock formations generally is to the north while movement in the lower part of the bedrock formations generally is to the west. Calculated rates of ground-water movement, using assumed values for hydraulic conductivity and porosity and the hydraulic gradients in the study area, indicate that the mean-lateral velocity in the alluvium is 380 feet per year $(120 \mathrm{~m} / \mathrm{yr})$ and is 27 feet per year $(8.2 \mathrm{~m} / \mathrm{yr})$ in the upper part of the bedrock formations. The calculated rate of vertical movement was 0.58 feet per year $(0.18 \mathrm{~m} / \mathrm{yr})$ in the upper part of the bedrock formations. Data on both the direction and the rate of ground-water movement indicate that lateral movement is predominant with minimal vertical groundwater movement.

Sampling of wells perforated in the alluvium, the upper part of the bedrock, and lower part of the bedrock indicated that water of better general quality is found in the deeper formations in the area. This suggests that the primary source of the water at depth in the area is not the downward movement of water from the alluvium but lateral movement from areas to the south and east.

Five wells perforated in alluvial materials were found to have water of markedly degraded quality. One well is perforated at the base of buried refuse in the landfill. Two other wells are located immediately downslope of two sludge-burial areas. The final two wells are used to water stock along Senac Creek. The degraded water in these wells may be due to the stock-tank 
overflow which forms nearby stagnant manure-contaminated ponds. Samples from most of the wells below the plowed disposal fields indicated that the water in the alluvium in these areas had nitrate concentrations (as $\mathrm{NO}_{3}$ ) ranging from 0.4 to $13 \mathrm{mg} / \mathrm{L}$. The concentrations are higher than the background nitrate concentration $(0.1 \mathrm{mg} / \mathrm{L})$ and are probably due to the sludge-disposal activities in the adjacent fields. Other wells in the alluvium and wells perforated in the upper part of the bedrock formations show no discernible effects of waterquality degradation produced by the waste-disposal activities in the area. Wells perforated in the lower part of the bedrock formations show no effects of the waste-disposal activities.

Because of continuing waste-disposal activities, continued water-quality monitoring is needed to determine the effects of the waste disposal on the quality of ground water, the only source of usable water in the area. It is suggested that all wells near the landfill be sampled twice a year with analysis for dissolved solids, sodium chloride, nitrate, trace metals, and phenolic compounds, and wells near the sludge-disposal areas be sampled on an annual basis with analysis for nitrate, ammonia, chloride, zinc, magnesium, sodium, and dissolved solids.

\section{REFERENCES}

Hughes, J. L., and Robson, S. G., 1973, Effects of waste percolation on groundwater in alluvium near Barstow, California, in Underground waste management and artificial recharge--A symposium: Am. Assoc. Petroleum Geologists, v. 1, p. 91-129.

McConaghy, J. A., Chase, G. H., Boettcher, A. J., and Major, T. J., 1964, Hydrogeologic data of the Denver Basin, Colorado: Colorado Water Conserv. Board Basic-Data Rept. 15, 224 p.

Palmquist, R., and Sedlein, V. A., 1975, The configuration of contamination enclaves from refuse disposal sites on floodplains: Ground Water, v. 13, no. 2 , p. 167-181.

Romero, J. C., 1976, Ground-water resource of the bedrock aquifers of the Denver Basin, Colorado: Colorado Dept. Nat. Resources, Div. Water Resources, Office of the State Engineer, 109 p.

Todd, D. K., 1967, Ground water hydrology: New York, John Wiley \& Sons, Inc., $336 \mathrm{p}$.

U.S. Environmental Protection Agency, 1973, Water quality criteria, 1972: Washington, D.C., U.S. Govt. Printing Office, EPA-R3-73-003, 594 p.

Zanon, A. E., 1971, Ground-water pollution from sanitary landfills and refuse dump grounds--A critical review: Wisconsin Dept. Nat. Resources, Research Report 69, 43 p. 
SUPPLEMENTAL INFORMATION 
Table 1.--Description

[Type of lift: J, jet; $N$, none; $P$, piston; $S$, submersible.

$\mathrm{U}$, unused. Aquifer: A, all

\begin{tabular}{|c|c|c|c|c|c|}
\hline \multicolumn{2}{|c|}{$\begin{array}{l}\text { LOCAL WELL } \\
\text { NUMBER }\end{array}$} & OWNER OR USER & $\begin{array}{l}\text { OEPTH } \\
\text { OF } \\
\text { WELL } \\
\text { (FEET) }\end{array}$ & $\begin{array}{c}\text { DIAMETER } \\
\text { OF } \\
\text { CASING } \\
\text { (INCHES) }\end{array}$ & $\begin{array}{l}\text { YEAR } \\
\text { COM- } \\
\text { PLETED }\end{array}$ \\
\hline $\begin{array}{l}\text { SC- } \\
\text { SC- } \\
\text { SC- } \\
\text { SC- }\end{array}$ & $\begin{array}{l}4-65-19 C C C \\
4-65-2 B A A B \\
4-65-28 B B C \\
4-65-28 B D D\end{array}$ & $\begin{array}{l}\text { SEAWRIGHT } \\
M \text { ARMATO } \\
\text { E SMITH } \\
U \text { S GOVERNMENT }\end{array}$ & $\begin{array}{r}--- \\
635 \\
21 \\
20\end{array}$ & $\begin{array}{r}-- \\
4 \\
8 \\
2\end{array}$ & $\begin{array}{l}1971 \\
1954 \\
1974\end{array}$ \\
\hline $\begin{array}{l}\text { SC- } \\
\text { SC- } \\
\text { SC- } \\
\text { SC- } \\
\text { SC- }\end{array}$ & $\begin{array}{l}4-65-28 D C A \\
4-65-29 A A A \\
4-65-30 A A B \\
4-65-30 B D A \\
4-65-30 B D D\end{array}$ & $\begin{array}{l}\text { U S GOVERNMENT } \\
\text { SMITH } \\
M \text { SMITH } \\
\text { E RIPPE } \\
U \text { S GOVERNMENT }\end{array}$ & $\begin{array}{r}20 \\
33 \\
573 \\
300 \\
23\end{array}$ & $\begin{array}{l}2 \\
6 \\
4 \\
7 \\
2\end{array}$ & $\begin{array}{l}1974 \\
1971 \\
1974\end{array}$ \\
\hline $\begin{array}{l}\text { SC- } \\
\text { SC- } \\
\text { SC- } \\
\text { SC- } \\
\text { SC- }\end{array}$ & $\begin{array}{l}4-65-30 D C A \\
4-65-300 C D \\
4-65-300 D D \\
4-65-31 A A A \\
4-65-31 A C C\end{array}$ & $\begin{array}{lll}U & S & \text { GOVERNMENT } \\
U & S & \text { GOVERNMENT } \\
U & S & \text { GOVERNMENT } \\
U & S & \text { GOVERNMENT } \\
U & S & \text { GOVERNMENT }\end{array}$ & $\begin{array}{r}4 \\
18 \\
16 \\
11 \\
102\end{array}$ & $\begin{array}{l}2 \\
2 \\
2 \\
2 \\
2\end{array}$ & $\begin{array}{l}1974 \\
1974 \\
1974 \\
1974 \\
1975\end{array}$ \\
\hline $\begin{array}{l}\text { SC- } \\
\text { SC- } \\
\text { SC- } \\
\text { SC- } \\
\text { SC- }\end{array}$ & $\begin{array}{l}4-65-31 C B A \\
4-65-31 D B B \\
4-65-31 D D C \\
4-65-32 A D A \\
4-65-32 A D D\end{array}$ & $\begin{array}{l}\text { CITY OF DENVER } \\
U \text { S GOVERNMENT } \\
U \text { S GOVERNMENT } \\
U \text { S GOVERNMENT } \\
U \text { S GOVERNMENT }\end{array}$ & $\begin{array}{r}902 \\
23 \\
28 \\
27 \\
21\end{array}$ & $\begin{array}{l}7 \\
2 \\
2 \\
2 \\
2\end{array}$ & $\begin{array}{l}1975 \\
1974 \\
1974 \\
1974 \\
1974\end{array}$ \\
\hline $\begin{array}{l}\text { SC- } \\
\text { SC- } \\
\text { SC- } \\
\text { SC- } \\
\text { SC- }\end{array}$ & $\begin{array}{l}4-65-32 B A B \\
4-65-32 C B C \\
4-65-32 D B B 1 \\
4-65-32 D B B 2 \\
4-65-320 B B 3\end{array}$ & 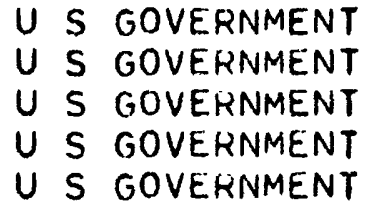 & $\begin{array}{r}11 \\
16 \\
41 \\
151 \\
248\end{array}$ & $\begin{array}{l}2 \\
2 \\
2 \\
2 \\
2\end{array}$ & $\begin{array}{l}1974 \\
1974 \\
1975 \\
1975 \\
1975\end{array}$ \\
\hline $\begin{array}{l}\text { SC- } \\
\text { SC- } \\
\text { SC- } \\
\text { SC- } \\
\text { SC- }\end{array}$ & $\begin{array}{l}4-65-33 B A B 1 \\
4-65-33 B A B 2 \\
4-65-33 B A B 3 \\
4-65-33 C B C \\
4-65-34 C B B\end{array}$ & $\begin{array}{l}U \text { S GOVERNMENT } \\
U \text { S GOVERNMENT } \\
U S \text { GOVERNMENT } \\
\text { STATE OF COLO } \\
U \text { S GOVERNMENT }\end{array}$ & $\begin{array}{l}28 \\
82 \\
23 \\
21 \\
16\end{array}$ & $\begin{array}{l}2 \\
2 \\
2 \\
6 \\
2\end{array}$ & $\begin{array}{l}1975 \\
1975 \\
1974\end{array}$ \\
\hline
\end{tabular}


of welzs

Use of water: $C$, commercial; $\mathrm{H}$, domestic; S, stock; uvium; B, bedrock]

\begin{tabular}{|c|c|c|c|c|c|}
\hline $\begin{array}{l}\text { ALTITUDE } \\
\text { OF LAND } \\
\text { SURFACE } \\
\text { (IN FEET } \\
\text { ABOVE M.S.L.) }\end{array}$ & $\begin{array}{l}\text { DEPTH } \\
\text { TO } \\
\text { WATER } \\
\text { (FEET) }\end{array}$ & $\begin{array}{l}\text { DATE } \\
\text { MEASURED }\end{array}$ & $\begin{array}{c}\text { TYPE } \\
\text { OF } \\
\text { LIFT }\end{array}$ & $\begin{array}{l}\text { USE } \\
\text { OF } \\
\text { WATER }\end{array}$ & AQUIFER \\
\hline
\end{tabular}

5628

5700

5628

5635

5642

5624

5670

5655

5640

5660

5660

5680

5683

5690

5710

5680

5710

5696

5694

5720

5720

5800

5800

5800

5670

5670

5665

5704

5665

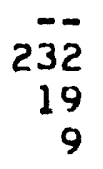

1974
1974
1975

7
18
217
149
10

1975

1974

1974

1974

1975

$\begin{array}{rl}-- & 1975 \\ 8 & 1975 \\ 12 & 1975 \\ 8 & 1975 \\ 14 & 1975\end{array}$

185

4

8

16

16

10

13

$12 \overline{2}$

122

16

14

10

10
1975

1975

1975

1975

1975

1975

1975

1975

1975

1975

1975

1975

1975

1974

1975

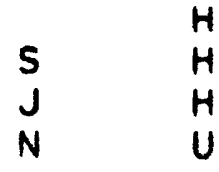

B

A

A

$\begin{array}{ll}N & U \\ S & H \\ S & H \\ P & S \\ N & U\end{array}$

A

A

B

B

A

$\begin{array}{ccc}N & U & A \\ N & U & A \\ N & U & A \\ N & U & A \\ N & U & B\end{array}$

S

N

C

U

U

U

U

A

A

A

B

B

A

A

A

A

$\begin{array}{ll}N & U \\ N & U \\ N & U \\ N & U \\ N & U\end{array}$

A
$A$
$B$
$B$
$B$

$\begin{array}{ll}N & U \\ N & U \\ N & U \\ P & S \\ N & U\end{array}$

A
$B$
$A$
$A$
$A$ 
Table 1.--Description

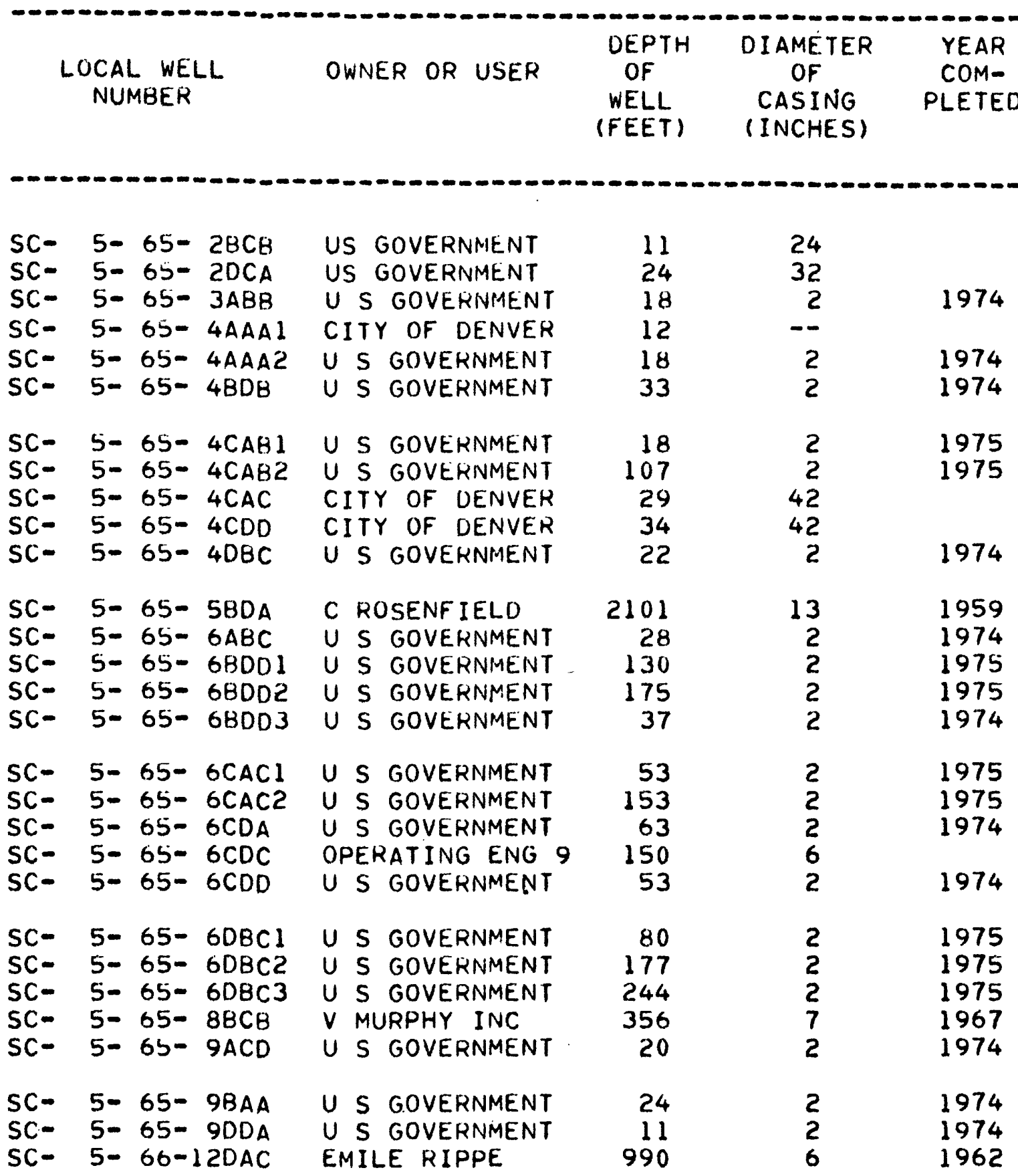


of werls--Continued

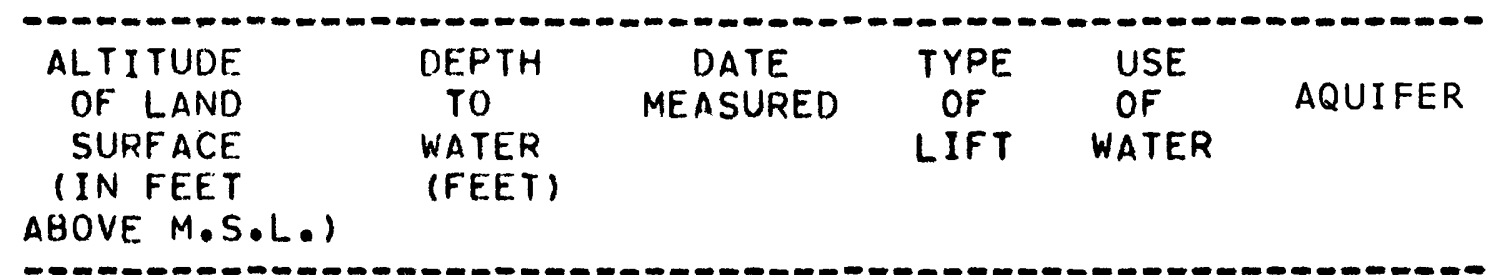

\begin{tabular}{|c|c|c|c|c|}
\hline $\begin{array}{l}5715 \\
5748 \\
5690 \\
5730 \\
5720 \\
5725\end{array}$ & $\begin{array}{r}9 \\
12 \\
6 \\
11 \\
10 \\
12\end{array}$ & $\begin{array}{l}1974 \\
1974 \\
1975 \\
1975 \\
1975 \\
1975\end{array}$ & $\begin{array}{l}P \\
P \\
N \\
N \\
N \\
N\end{array}$ & $\begin{array}{l}S \\
S \\
U \\
U \\
U \\
U\end{array}$ \\
\hline $\begin{array}{l}5745 \\
5745 \\
5748 \\
5760 \\
5750\end{array}$ & $\begin{array}{l}13 \\
26 \\
18 \\
20 \\
12\end{array}$ & $\begin{array}{l}1975 \\
1975 \\
1974 \\
1974 \\
1975\end{array}$ & $\begin{array}{l}N \\
N \\
P \\
N \\
N\end{array}$ & $\begin{array}{l}u \\
u \\
S \\
u \\
u\end{array}$ \\
\hline $\begin{array}{l}5812 \\
5725 \\
5757 \\
5757 \\
5755\end{array}$ & $\begin{array}{r}205 \\
9 \\
44 \\
47 \\
9\end{array}$ & $\begin{array}{l}1959 \\
1975 \\
1975 \\
1975 \\
1975\end{array}$ & $\begin{array}{l}S \\
N \\
N \\
N \\
N\end{array}$ & $\begin{array}{l}H \\
U \\
U \\
U \\
U\end{array}$ \\
\hline $\begin{array}{l}58.03 \\
5803 \\
5833 \\
5838 \\
5835\end{array}$ & $\begin{array}{l}32 \\
79 \\
51 \\
67 \\
31\end{array}$ & $\begin{array}{l}1975 \\
1975 \\
1975 \\
1974 \\
1975\end{array}$ & $\begin{array}{l}N \\
N \\
N \\
S \\
N\end{array}$ & $\begin{array}{l}U \\
U \\
U \\
H \\
U\end{array}$ \\
\hline $\begin{array}{l}5817 \\
5817 \\
5817 \\
5800 \\
5770\end{array}$ & $\begin{array}{l}77 \\
99 \\
83 \\
37 \\
17\end{array}$ & $\begin{array}{l}1975 \\
1975 \\
1975 \\
1974 \\
1975\end{array}$ & $\begin{array}{l}N \\
N \\
N \\
P \\
N\end{array}$ & $\begin{array}{l}u \\
u \\
u \\
S \\
u\end{array}$ \\
\hline $\begin{array}{l}5740 \\
5810 \\
5900\end{array}$ & $\begin{array}{r}8 \\
3 \\
384\end{array}$ & $\begin{array}{l}1975 \\
1975 \\
1962\end{array}$ & $\begin{array}{l}N \\
N\end{array}$ & $\begin{array}{l}U \\
U \\
H\end{array}$ \\
\hline
\end{tabular}




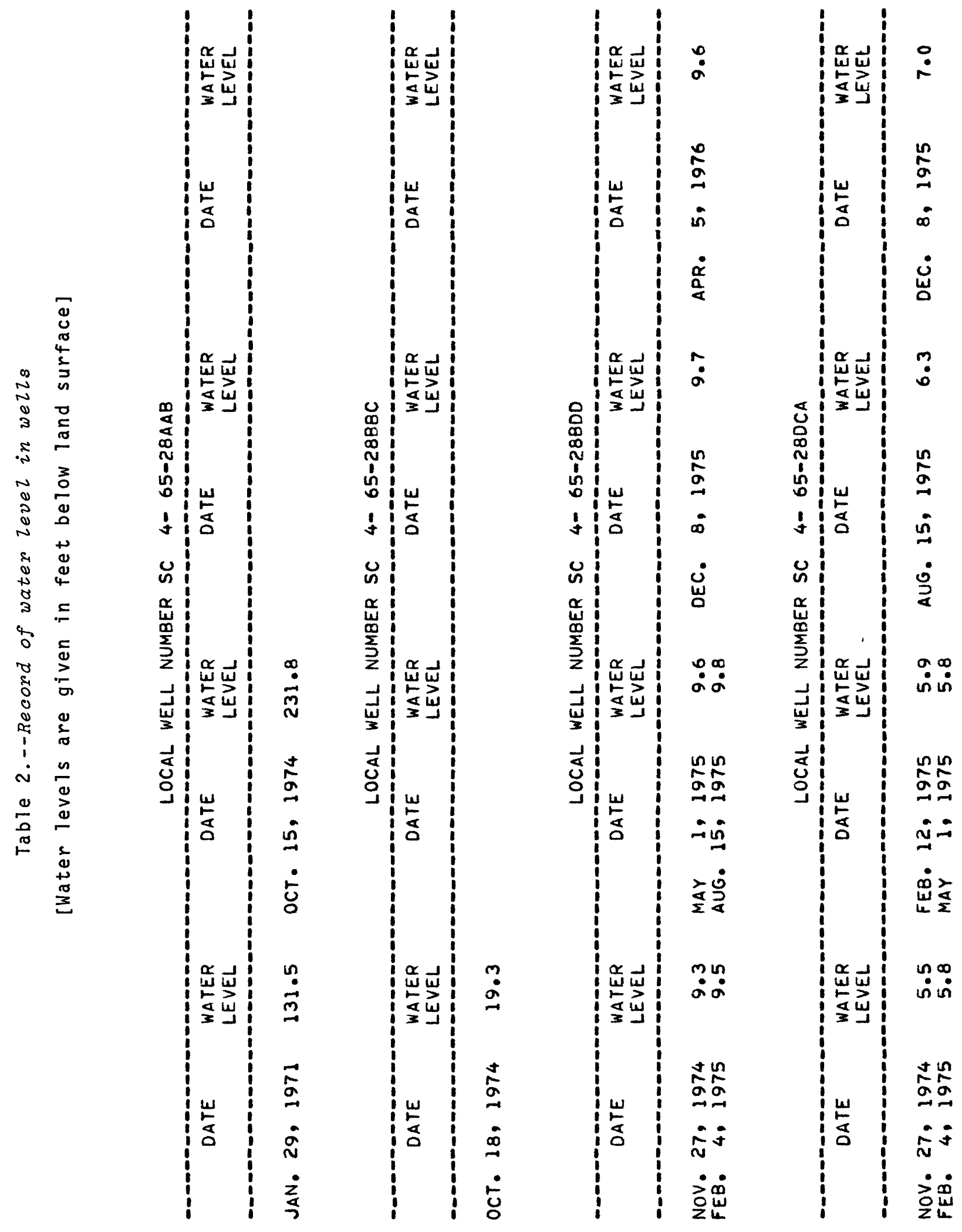




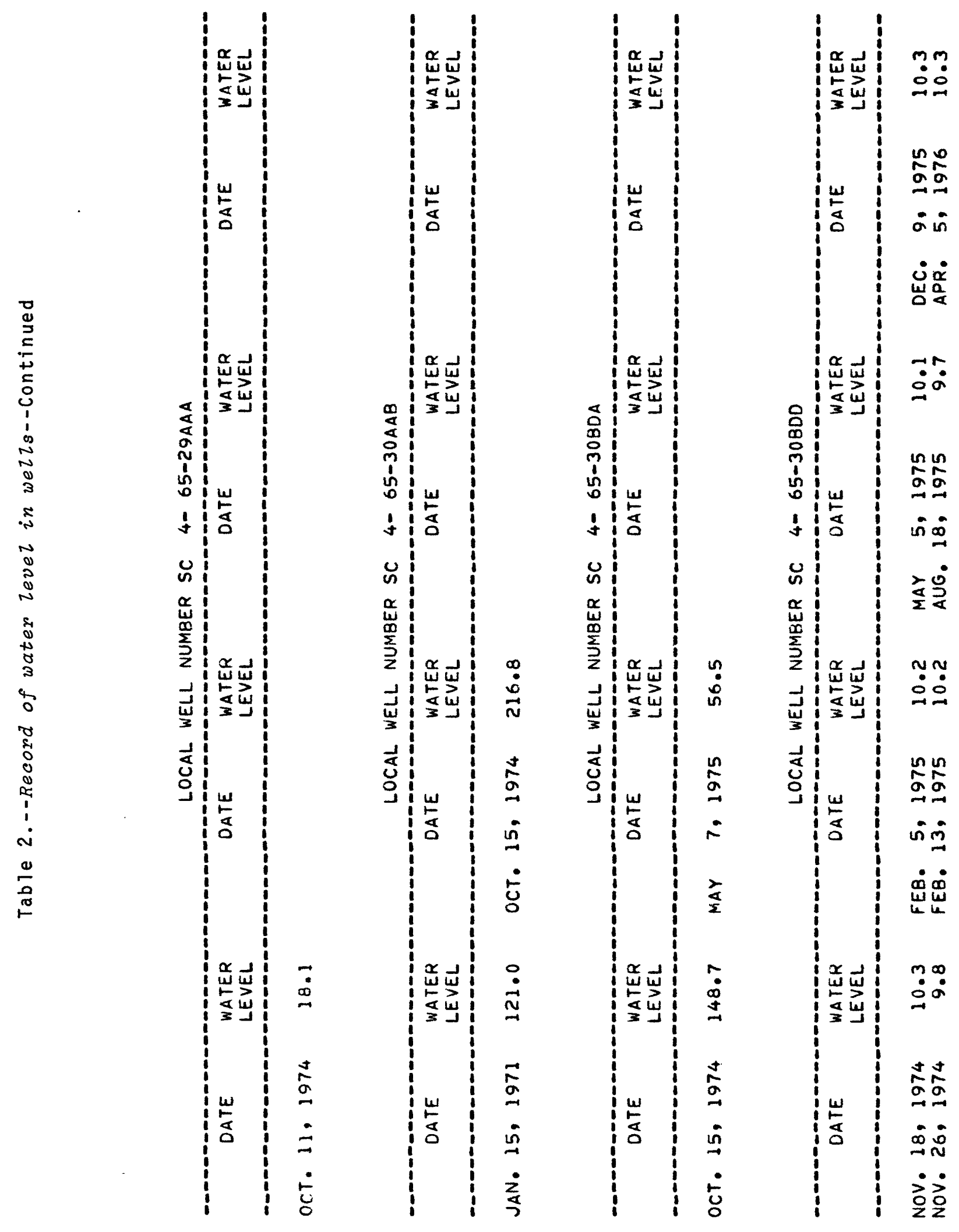




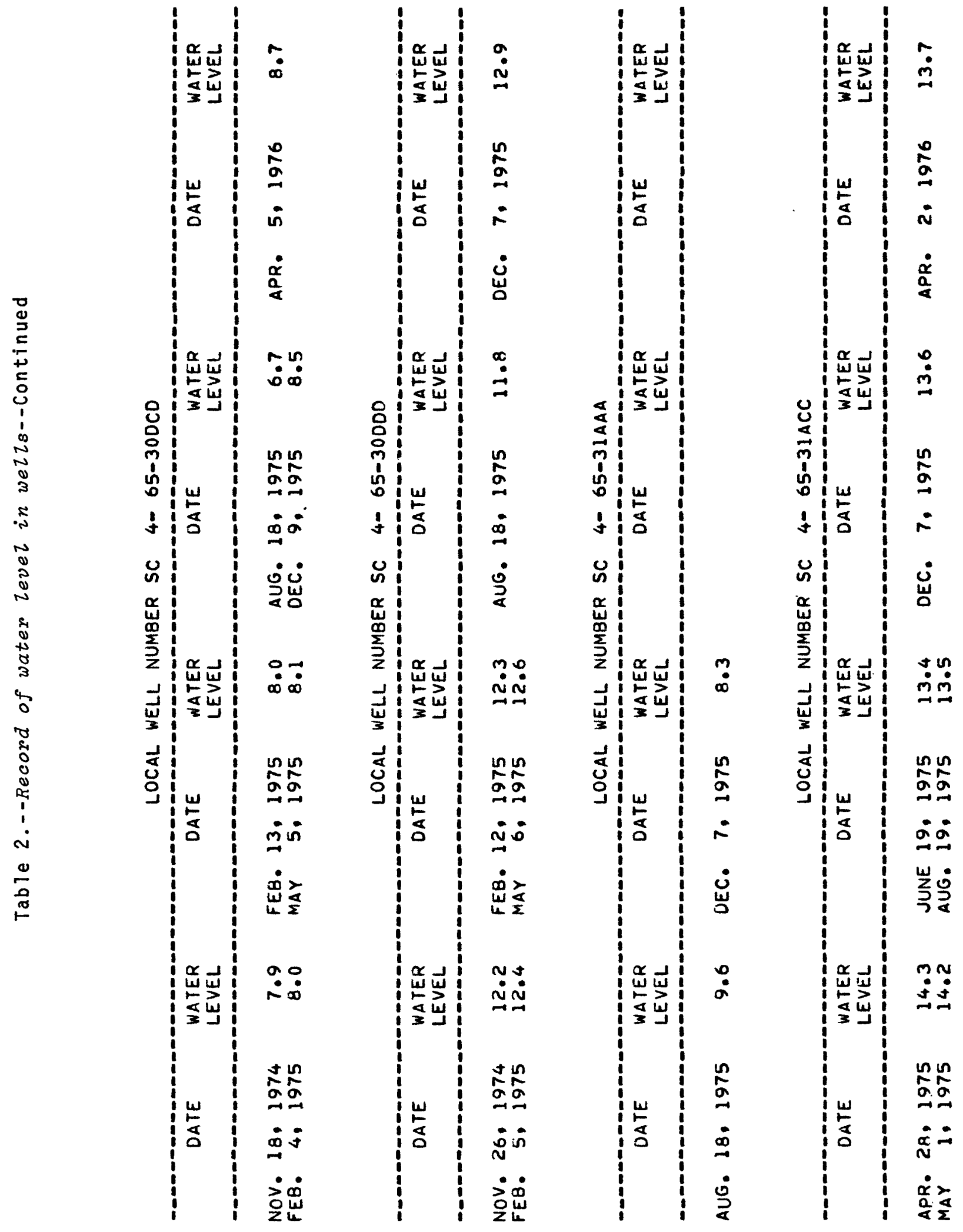




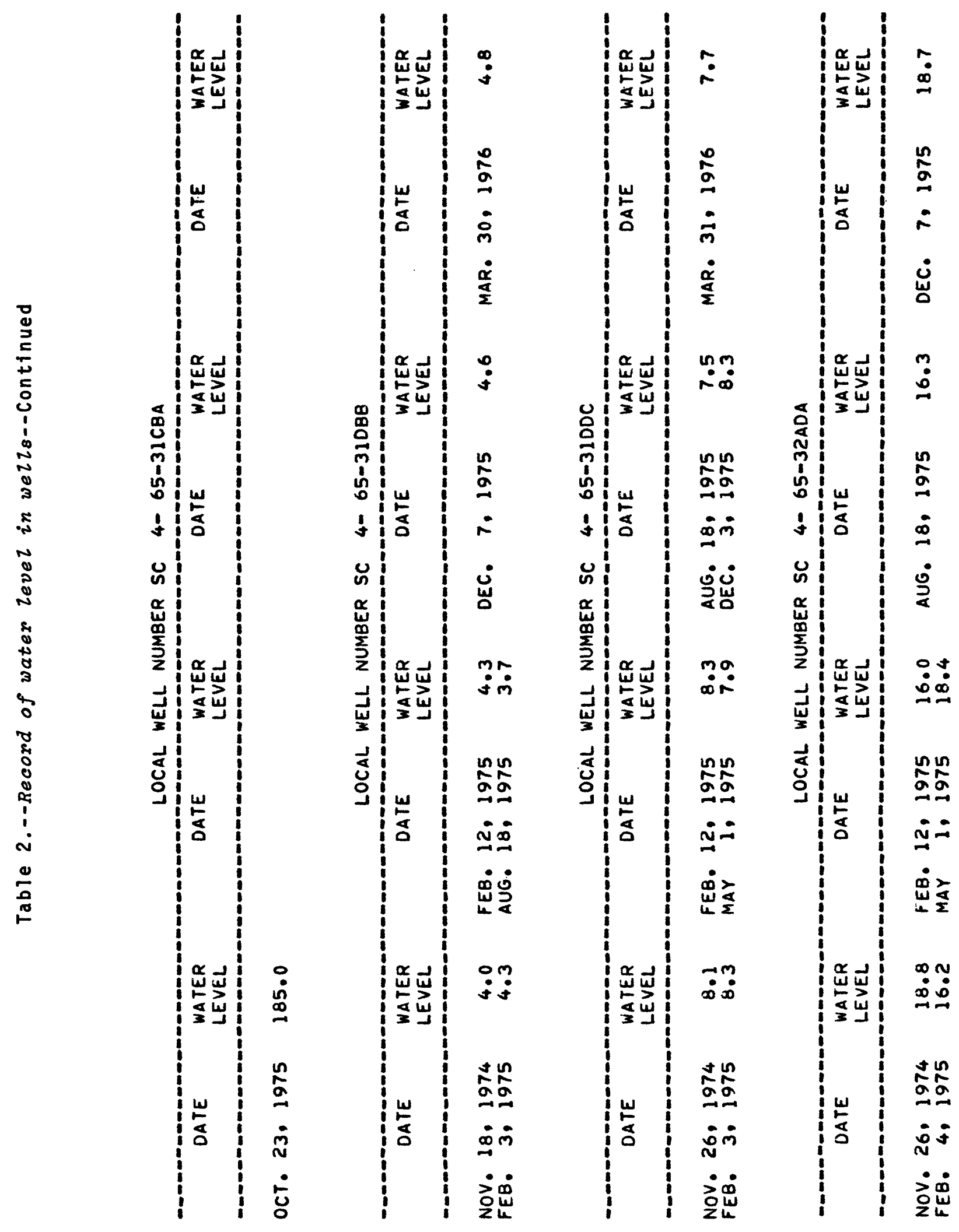



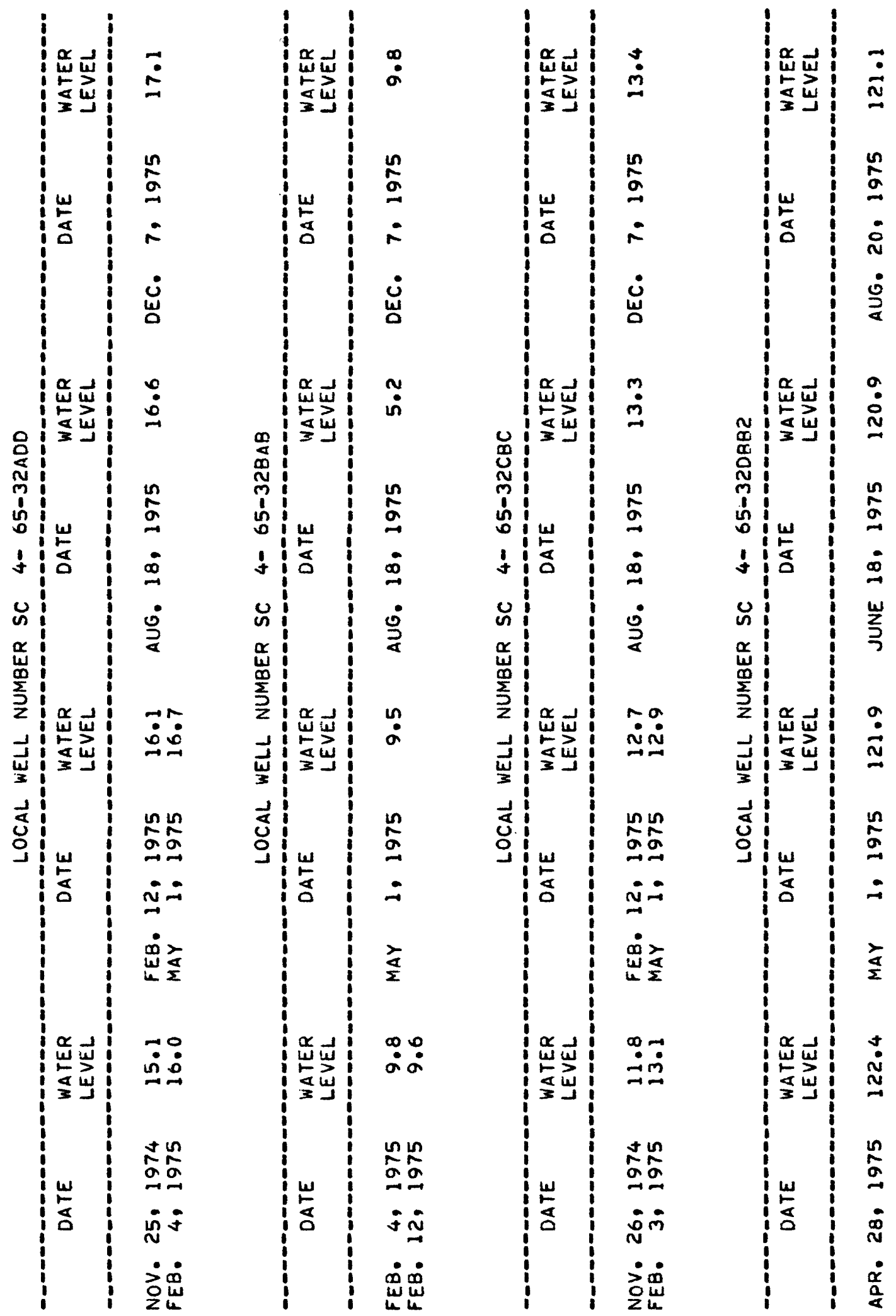


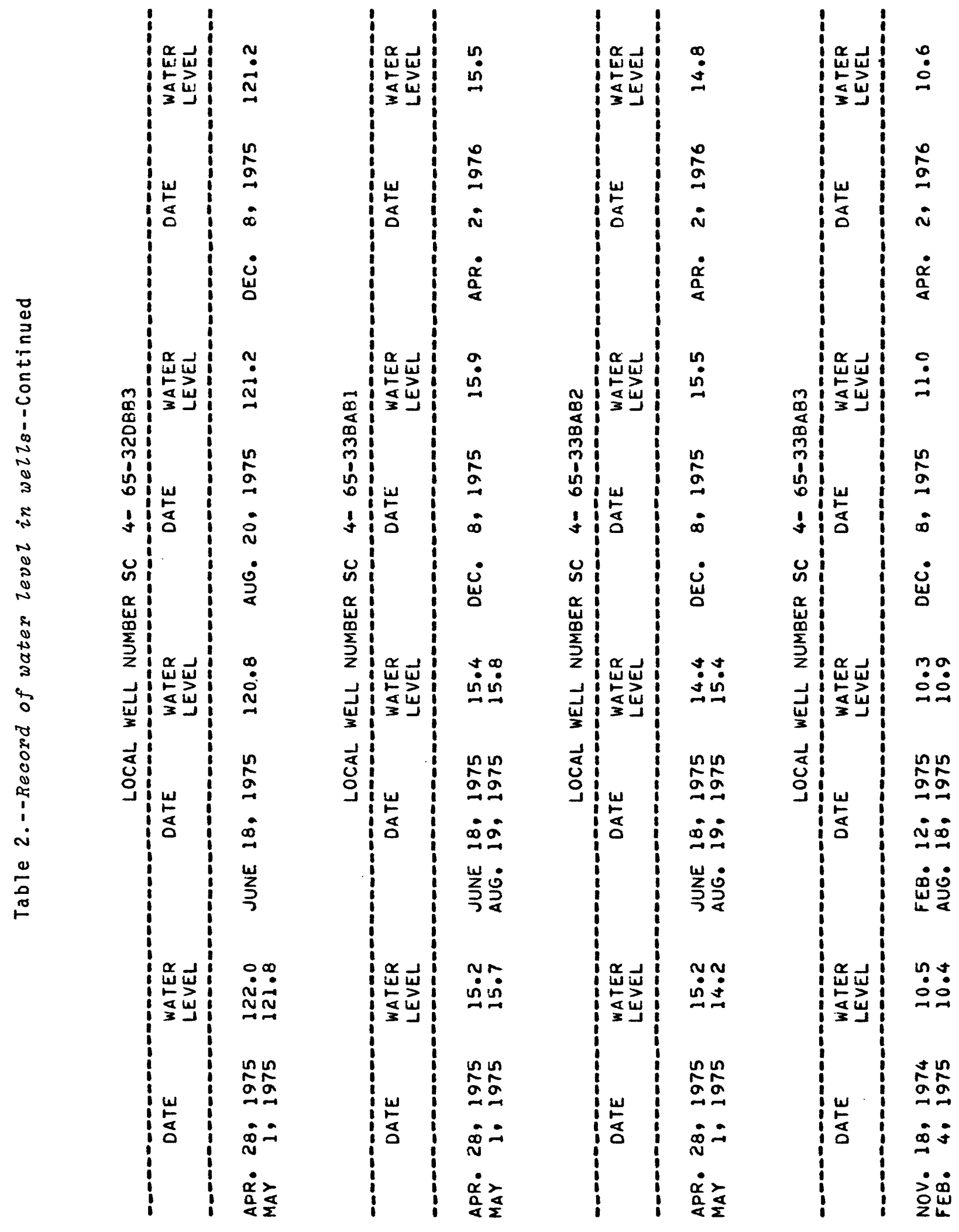




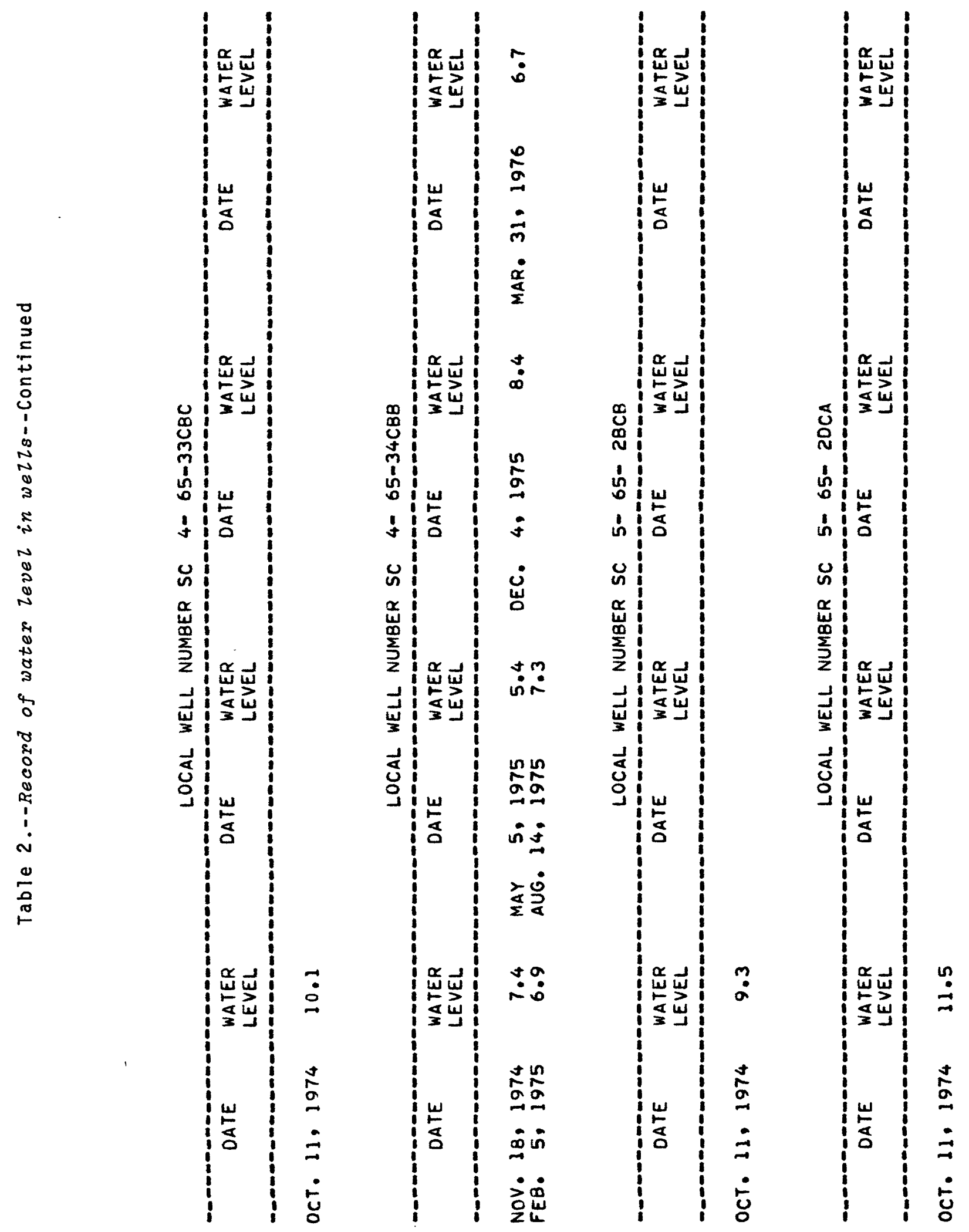




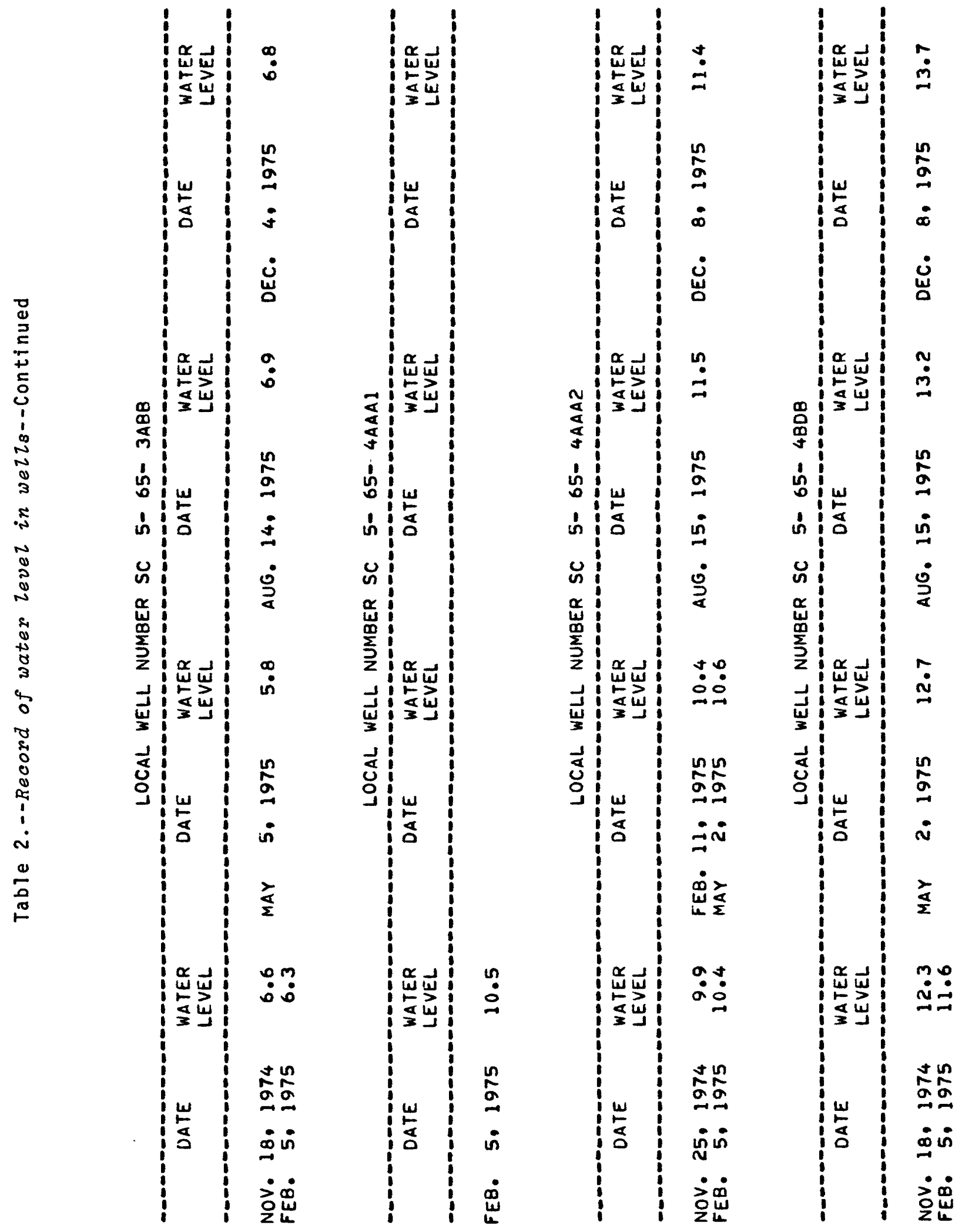




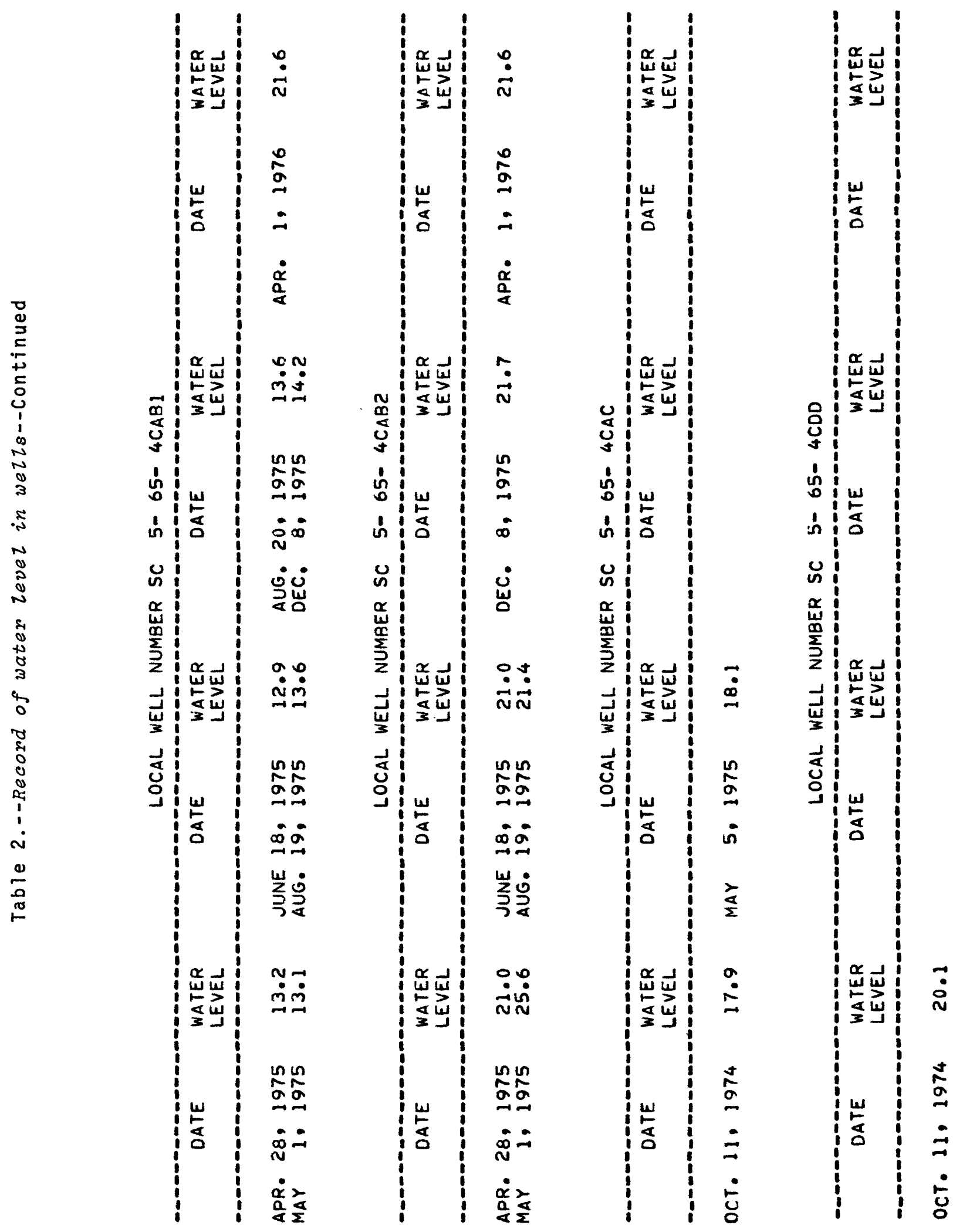




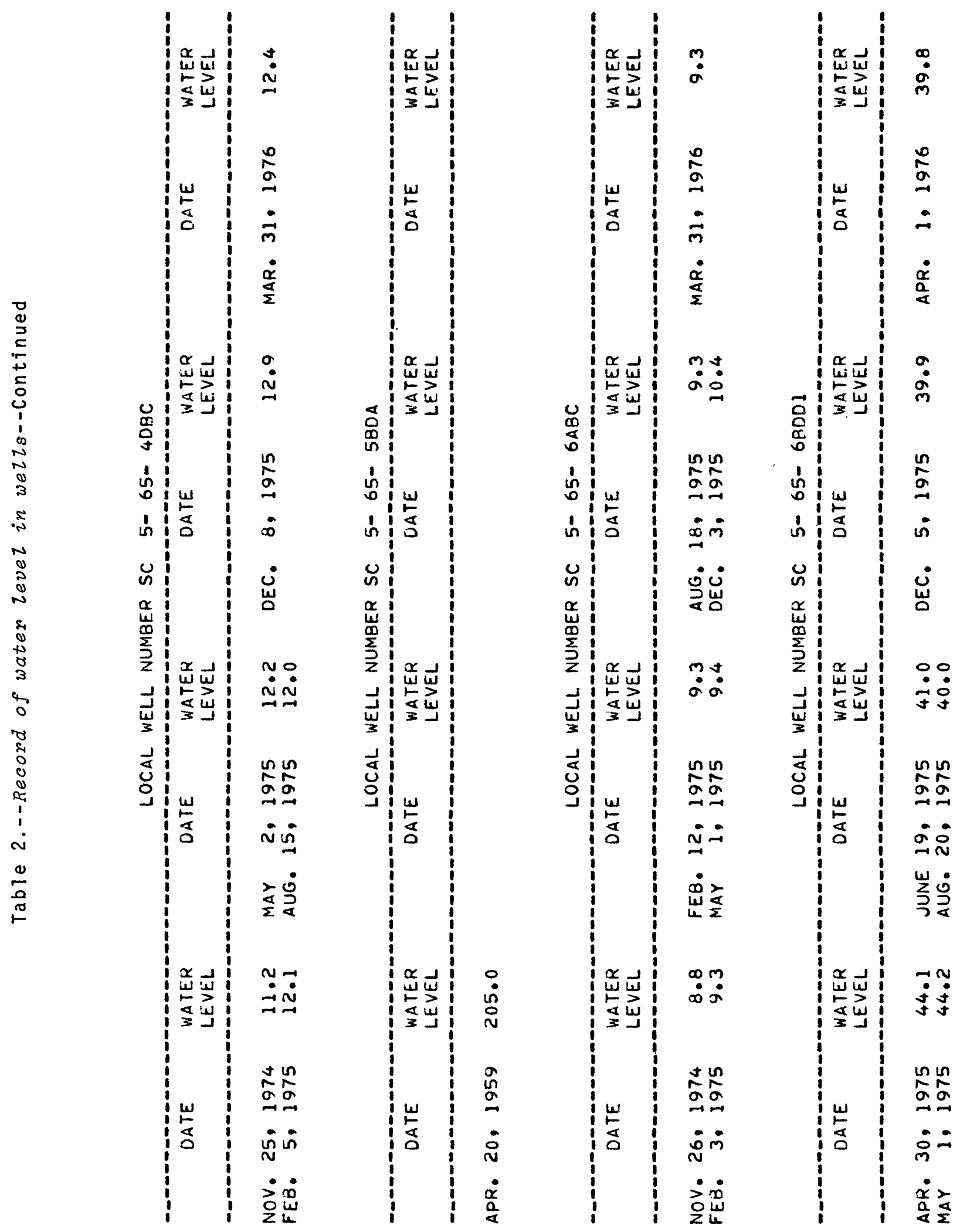




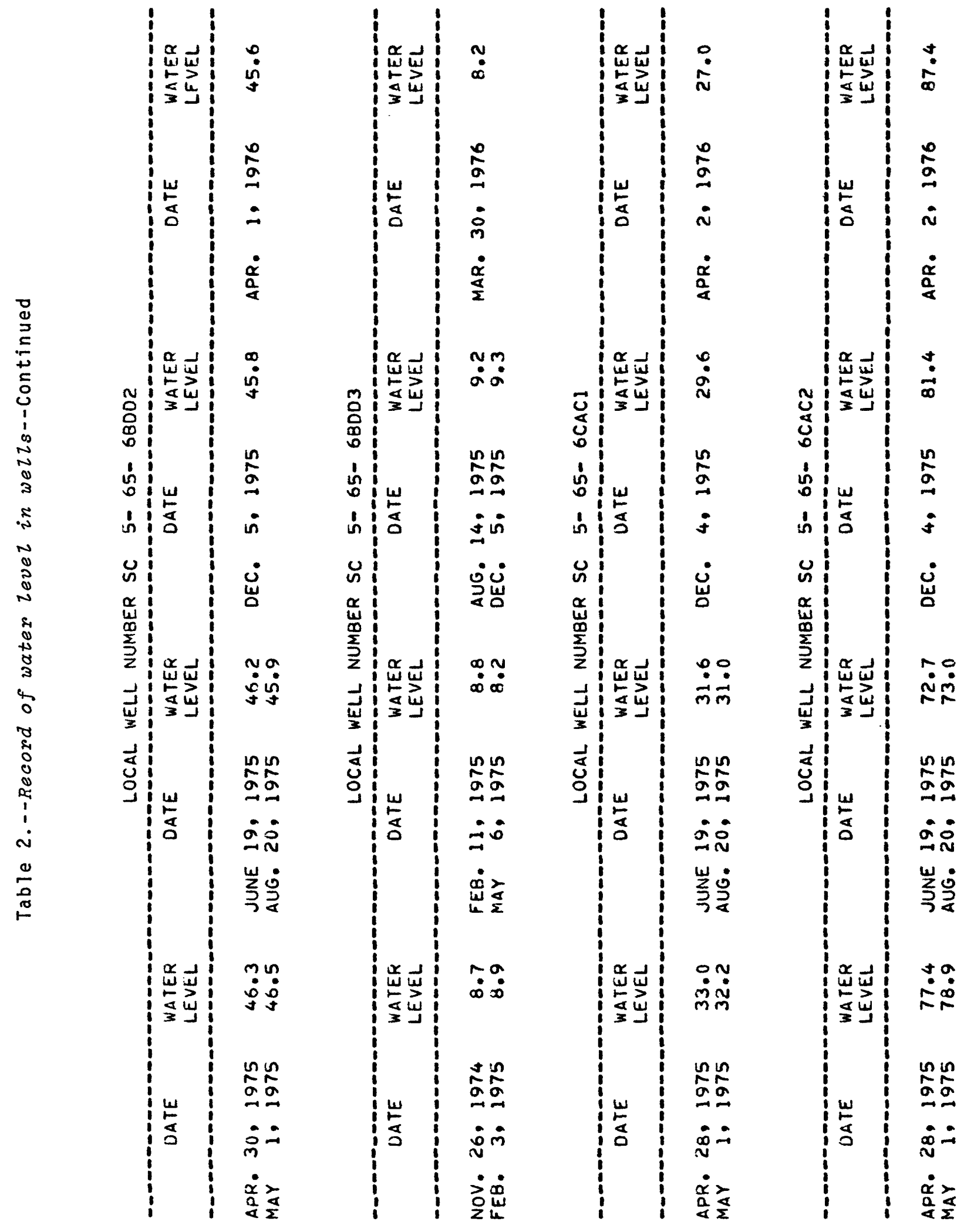




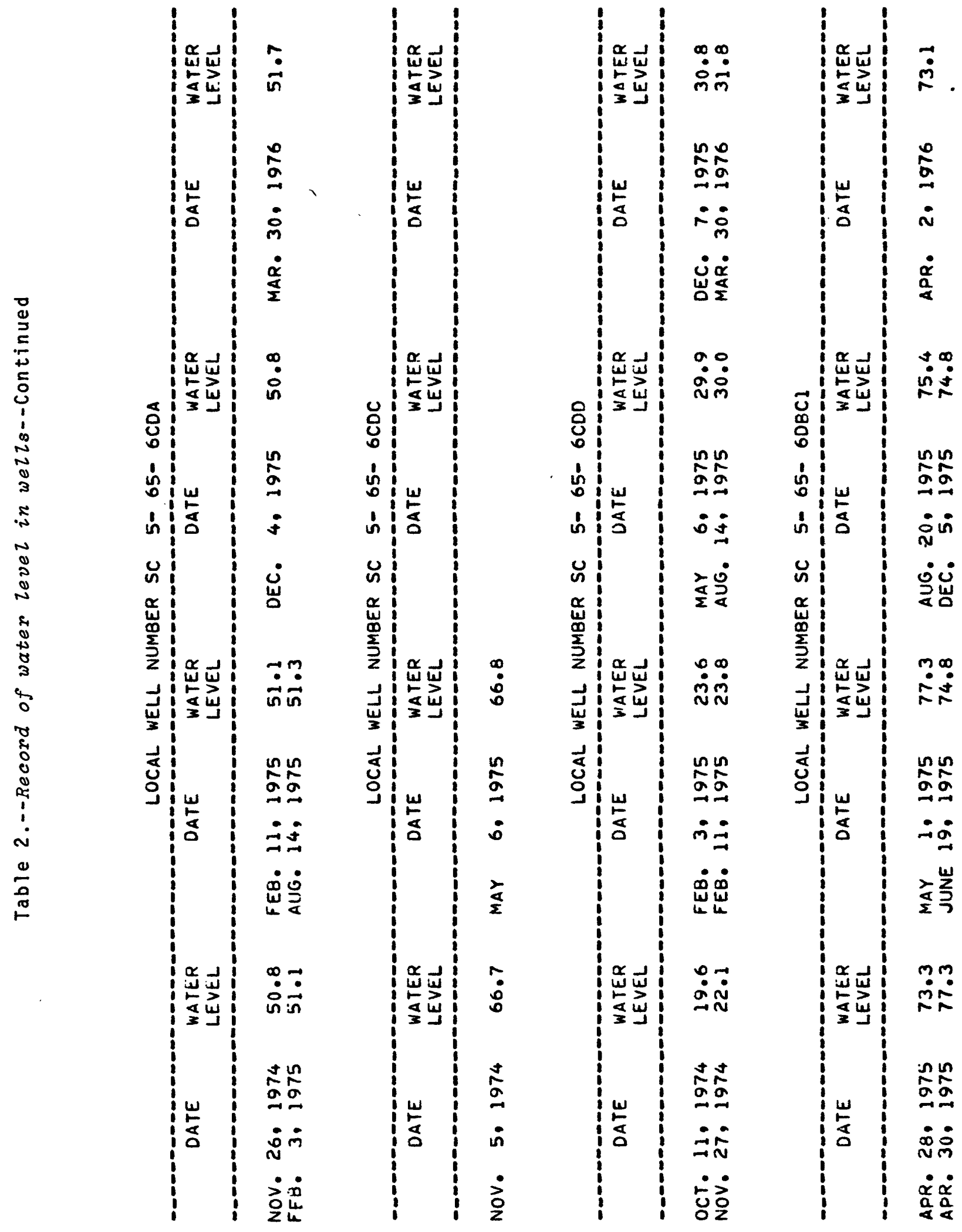




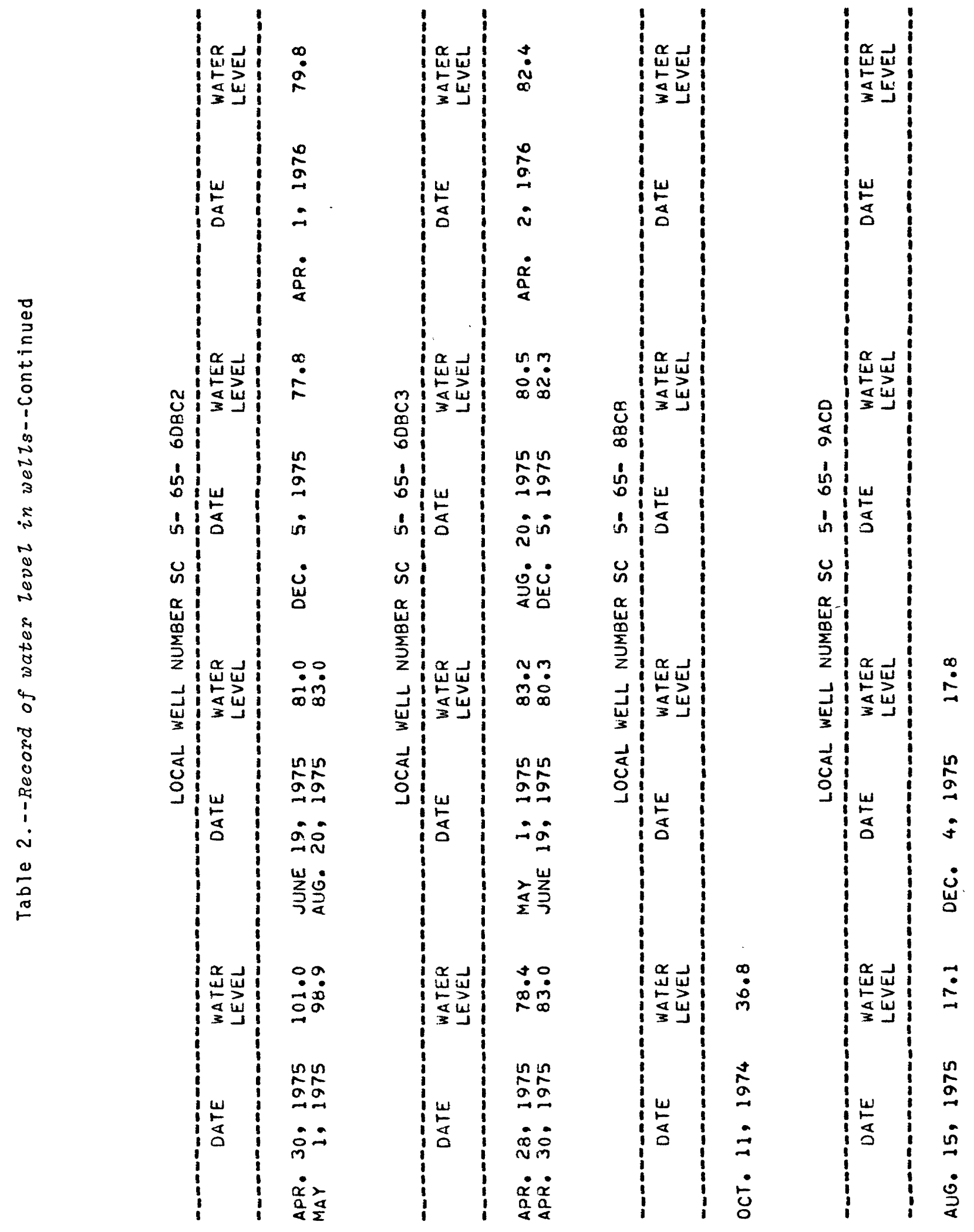




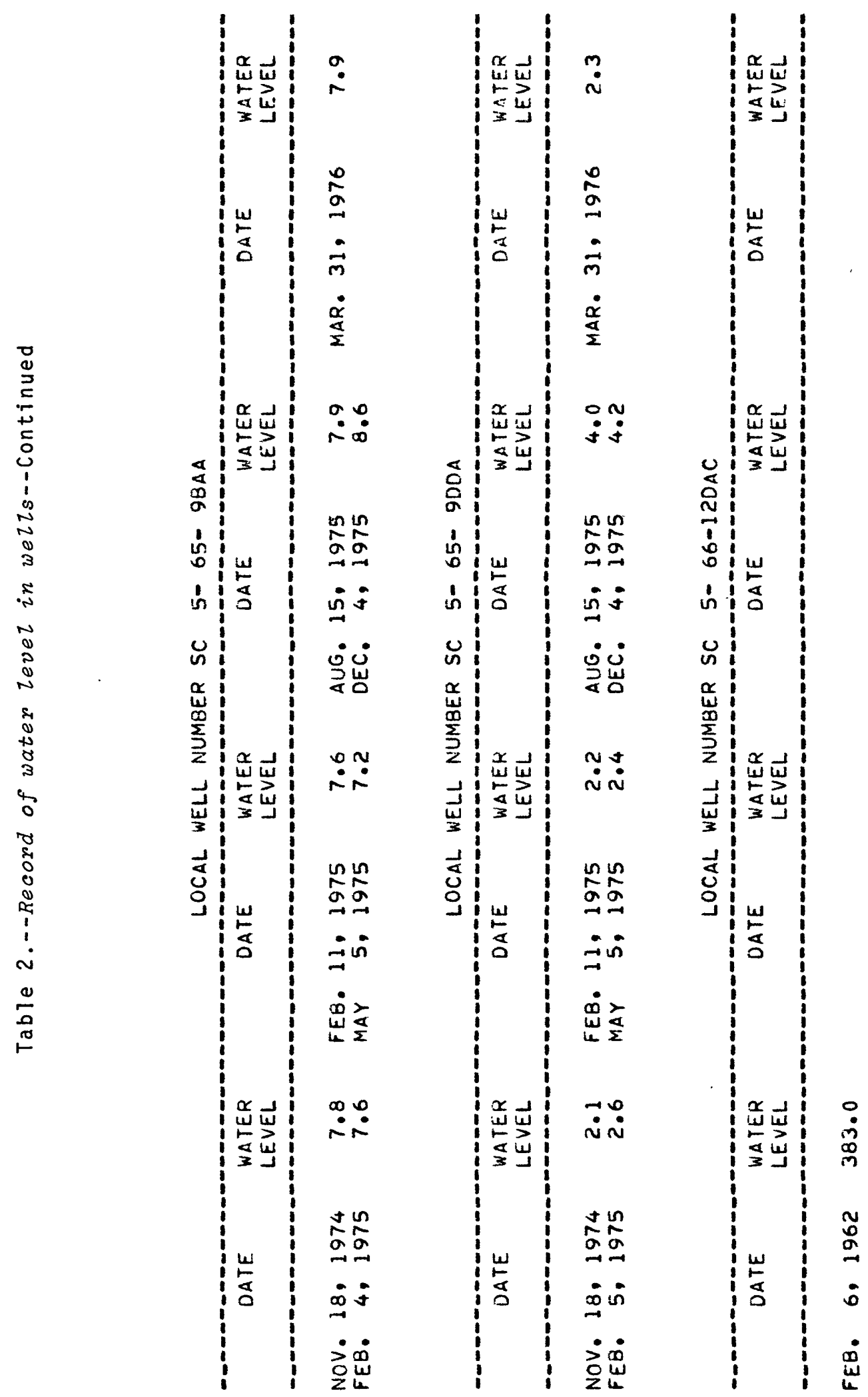


Table 3.--Logs of wells drilled by the the U.S. Geological Survey

\begin{tabular}{ccc}
\hline & $\begin{array}{c}\text { Thick- } \\
\text { ness } \\
\text { (feet) }\end{array}$ & $\begin{array}{c}\text { Depth } \\
\text { (feet) }\end{array}$ \\
\hline
\end{tabular}

We11 SC 4-65-28BDD. Altitude, 5,635 feet

Sand, fine, with a little silt, brown-_-_-_-_-_--

Sand grading from medium to coarse, well-sorted--------

Sand, very coarse, well-sorted--_--

Sand, very coarse, with silt-_-

Clay, silty, blue-gray

We11 SC 4-65-28DCA. A1titude, 5,642 feet

Sand, fine, well-sorted-

Sand, fine to coarse, well-sorted

Sand, coarse, well-sorted--_-_--

Clay, silty, blue-gray

We11 SC 4-65-30BDD. A1titude, 5,640 feet

Sand, poorly sorted, brown--

Sand and clay, brown

We11 SC 4-65-30DCA. Altitude, 5,660 feet

Sand, fine--_-

Sandstone, we11-consolidated

We11 SC 4-65-30DCD. A1titude, 5,660 feet

Sand, poorly sorted, brown--_-_-

Grave1, small, and sand, poorly sorted--_-----

Sand, poorly sorted, tougher drilling

5

We11 SC 4-65-30DDD. Altitude, 5,680 feet

Sand, fine, and silt, brown--

Sandstone and siltstone; color grades from yellowbrown to brown--

Clay, silty-_-

Sandstone, wel1-consolidated, blue-gray-_-_-_-_-_-_-_ 
Table 3.--Logs of wells drilled by the U.S. Geological Survey--Continued

Thick-

ness

(feet)

We11 SC 4-65-31AAA. Altitude, 5,683 feet

Sand, very fine, silty-_._-

Grave1, coarse, with silty sand-_-_.-.

Clay, with silt and fine sand

We11 SC 4-65-31ACC. Altitude, 5,690 feet

Silt, dark-brown--

Siltstone; alternating layers are either gray or tan---

Sand and gravel, with thin layers of light-tan clay----

Siltstone grading from yellow-brown to blue-gray-------

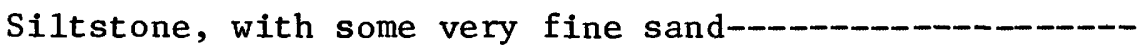

Siltstone, blue-gray

$12 \quad 18$

$7 \quad 25$

$10 \quad 35$

$3 \quad 38$

$62 \quad 100$

Siltstone with a few small seams of coal-_-_-_- $\quad 20 \quad 120$

Siltstone--_-_-_- $\quad 31$

Coal and siltstone layers-_-_-_- $\quad 2 \quad 153$

Siltst one--_-_-_-_-

Coal and siltstone layers-_-

Siltstone, gray grading to blue-gray-_-_-_-_-_-_-_ 10

Coal-_-_--_-_-

Siltstone, with a few thin seams of coal; hole yields about $2 \mathrm{gal} / \mathrm{min}-\ldots$

Siltstone, blue-gray-_-_-_-_-_ 20

200

$20 \quad 220$

We11 SC 4-65-31DBB. Altitude, 5,680 feet

Sand, medium, with some silt, dark-brown-_.......-..--

Sand, very coarse, poorly sorted-_-_-

Silt and sand-

Silt and sand in alternating layers; silt contains pebbles of hard blue-gray siltstone- 
Table 3.--Logs of wells drilZed by the U.S. Geological Survey--Continued

Thickness

(feet)

We11 SC 4-65-31DDC. Altitude, 5,710 feet

Sand, poorly sorted, brown- 5

Sand and silt-

Clay, silty, yellow-brown-

Clay, silty, grading to blue-gray

We11 SC 4-65-32ADA. Altitude, 5,696 feet

Silt with fine sand, brown--

Clay, silty; color grading from brown to yellow-brown--

Clay, silty, blue-gray

We11 SC 4-65-32ADD. Altitude, 5,694 feet

Clay, silty, brown; hard drilling near bottom of hole--

We11 SC 4-65-32BAB. Altitude, 5,720 feet

Sand, very fine, grading to silty clay, light-brown----

We11 SC 4-65-32CBC. Altitude, 5,720 feet

Clay, silty, with fine sand, dark-brown-

Clay, silty, yellow-brown

We11 SC 4-65-32DBB1,2,3. Altitude, 5,800 feet

Silt and clay; color grading from brown to blue-gray--4

Sandstone, coarse, poorly consolidated, with a few

layers of buff siltstone; hole is dry--

Siltstone, yellow-brown--_-

21

328

Sandstone, fine, with thin layers of silt, yellow-brown 8

Conglomerate, coarse, well-consolidated; hole is dry---

Siltstone and fine sand, yellow-brown--

Claystone, dense, gray-brown; hole is dry------------Siltstone, dense, with layers of silt or very fine sand 
Table 3.--Logs of wells drilled by the U.S. Geological Survey--Continued

Thickness

(feet)

We11 SC 4-65-32DBB1,2,3--Continued

Sandstone, fine to medium, moderately consolidated-----

Sandstone, medium, very well consolidated--_----_------

Sandstone, fine to medium, poorly consolidated---------

Claystone, alternating layers are either blue-gray or brown; hole yields 2 to $4 \mathrm{gal} / \mathrm{min}-$

Siltstone, poorly consolidated, blue-gray, with a few thin seams of coal-

Shale, brown, with thin seams of coal---_--_------

Claystone, blue-green, with a few thin seams of coal--Coa1

$\begin{array}{rr}6 & 200 \\ 5 & 205 \\ 41 & 246 \\ 8 & 254\end{array}$

Claystone, brown, with coal seams; hole yields about

2 to $4 \mathrm{gal} / \mathrm{min}-$

We11 SC 4-65-33BAB1,2. Altitude, 5,670 feet

Sand, coarse, with some silt and clay layers--------20 20

Sand and gravel, medium, with some silt and clay layers Siltstone, blue-gray--_--_-

Siltstone, brown, with thin layers of coal------------

Siltstone, blue-gray, with thin layers of well-consolidated sandstone--

Sandstone, with thin seams of coa1--

Siltstone-C-

Sand, gray; hole yields $30 \mathrm{gal} / \mathrm{min}$

Sand, coarse, with thin layers of silt---------------

Siltstone, blue-gray; hole yields about $30 \mathrm{gal} / \mathrm{min}-----$

Claystone, gray-----

Siltstone, brown, with thin seams of coal-----

Coa1------

Siltstone, blue-gray-----

$\begin{array}{rr}12 & 32 \\ 11 & 43 \\ 9 & 52 \\ 13 & 65 \\ 5 & 70 \\ 7 & 77 \\ 3 & 80 \\ 12 & 92 \\ 8 & 100 \\ 5 & 105 \\ 3 & 108 \\ 12 & 120 \\ 10 & 130\end{array}$


Table 3.--Logs of wells drilled by the U.S. Geological Survey--Continued

Thickness

(feet)

We11 SC 4-65-33BAB3. Altitude, 5,665 feet

Sand, very fine to coarse, brown- 5

Sand grading into silt, with sand and gravel--

Clay, silty, yellow-brown, with layers of very coarse grave1-

Clay, silty; color grading from brown to blue-gray----

We11 SC 4-65-34CBB. Altitude, 5,665 feet

Sand, fine, with some silt, light-brown--

Sand, fine to coarse-

Sand and silt, gray-brown-

Clay, silty, gray-green

We11 SC 5-65- 3ABB. 5,690 feet

Sand, fine, wel1-sorted, medium-brown-

Sand, fine, and gray silt-_

Sand and silt, gray

clay, silty, yellow-brown

We11 SC 5-65- 4AAA2. Altitude, 5,720 feet

Sand, coarse, wel1-sorted, light-brown-

Clay, silty, dark-brown-

Clay, silty, blue-gray

We11 SC 5-65-4BDB. Altitude, 5,725 feet

Sand, very fine, wel1-sorted$\begin{array}{ll}5 & 5 \\ 3 & 8\end{array}$

Clay, silty, dark-brown, with some medium sand-- - -

Sand, coarse, with alternating layers of gray-brown silty clay-_ 
Table 3.--Logs of wells drilled by the U.S. Geological Survey--Continued

Thick-

ness

(feet)

We11 SC 5-65- 4CAB1,2. Altitude, 5, 745 feet

Silt and clay, with some fine sand, medium-brown------

22

22

Siltstone, rust-color

3

25

Siltstone, blue-gray

6

31

Sandstone

1

32

Siltstone, blue-gray grading to brown, with a few smal1 coal seams--

8 40

Siltstone; alternating layers are blue gray or brown; hole yields about $10 \mathrm{gal} / \mathrm{min-}$

Siltstone; alternating blue-gray or brown layers------

Siltstone, with a few layers of very fine, poorly consolidated sand---

Siltstone, blue-green---

Sandstone, medium, gray-brown, with some coal seams----

Siltstone, with seams of coal and sandstone---------

Siltstone, blue-gray, with alternating layers of brown siltst one--

Coal

$\begin{array}{rr}48 & 183 \\ 13 & 196 \\ 4 & 200\end{array}$

Siltstone, gray-brown-

20

60

44

104

10

114

10 124

5 129

6 135

We11 SC 5-65- 4DBC. Altitude, 5,750 feet

Sand and silt, medium-brown

Clay, silty, ranging from gray-brown to red-brown to yellow-brown--------

We11 SC 5-65- 6ABC. Altitude, 5,725 feet

Clay, silty, grading from yellow-brown to red-brown---- 
Table 3.--Logs of wells drilled by the U.S. Geological Survey--Continued

Thick-

ness

(feet)

We11 SC 5-65-6BDD3. Altitude, 5,755 feet

Clay, sandy, medium brown-- 5

Clay, silty, grading from gray-tan to yellow-tan------

We11 SC 5-65- 6BDD1,2. Altitude, 5,757 feet

Mudstone, medium-brown, with some coarse sand--------

Clay; alternating layers are red brown or brown-------

Claystone, ye11ow-brown-

Siltstone, blue-gray

23

Siltstone and fine sandstone, poorly consolidated------

Siltstone, dark-brown, with traces of coal-

Siltstone, blue-gray

Shale, brown, with seams of sandstone-C-

Siltstone, blue-gray

Sandstone, fine, well-consolidated; hole yields about

Siltstone; alternating layers are blue gray or brown---

Sandstone, blue-gray grading to dark-brown; hole yields about $5 \mathrm{gal} / \mathrm{min}-$

Siltstone; alternating layers are blue gray or brown---

Sandstone, medium, wel1-consolidated-C-

6

Siltstone, gray, with small layers of sandstone and coa 1

Siltstone, blue-green; hole yields about $10 \mathrm{gal} / \mathrm{min----}$

We11 SC 5-65-6CAC1,2. Altitude, 5,803 feet

\section{Claystone, grading from buff to rust--}

Clay, sand, and small gravel-_-

Mudstone, blue-gray, with some sand---- 
Table 3.--Logs of wells drilled by the U.S. Geological Survey--Continued

$\begin{array}{cr}\begin{array}{c}\text { Thick- } \\ \text { ness }\end{array} & \text { Depth } \\ \text { (feet) } & \text { (feet) }\end{array}$

We11 SC 5-65- 6CAC1,2--Continued

Claystone, blue-green, with small amount of fine sand-7

Claystone, blue-gray, with some fine to medium sand and small coal seams; hole yields about $20 \mathrm{gal} / \mathrm{min-------}$

Shale, red-brown, with some medium sandstone--_------

Sandstone, moderately consolidated, blue-gray--.---.---

Sandstone, well-consolidated, with small coal seams;

hole yields about $30 \mathrm{gal} / \mathrm{min}$

Sandstone, with layers of shale, blue-gray-

Mudstone with sandstone layers-_-

Shale, brown, with layers of blue-gray mudstone; hole yields about $30 \mathrm{gal} / \mathrm{min}-$

We11 SC 5-65-6CDA. A1titude, 5,833 feet

Landfill refuse--_-

Smooth drilling; no return up hole; strong $\mathrm{H}_{2} \mathrm{~S}$ odor----

Clay, dense, light-brown; tough drilling-_-_-_-_-_---

We11 SC 5-65- 6CDD. A1titude, 5,835 feet

Reworked earth fill-_- 10

Sand, fine, and silt, yellow-brown-

Clay, silty, alternating layers are yellow brown or

blue gray

15

Clay, silty, red-brown-

Sandstone

Sand and silt, blue-gray-

We11 SC 5-65-6DBC1,2,3. Altitude, 5,817 feet

Mudstone, grading from buff to lighter brown--------

Claystone, with very little sand, light-brown--..--..--

Mud, red-brown- 
Table 3.--Logs of wells drilled by the U.S. Geological Survey--Continued

Thick-

ness

(feet)

We11 SC 5-65-6DBC1,2,3--Continued

Siltstone, with very little sand, gray-

Coa1

Siltstone and claystone, gray-green

Coal with layers of poorly consolidated sandstone;

hole yields about 2 gal/min-_-

Siltstone, with small seams of coal-

Coa 1

18

Claystone, gray; hole yields about $0.5 \mathrm{gal} / \mathrm{min}$

Siltstone, with layers of very fine, poorly consolidated sandstone, blue-gray-

Siltstone; color grading from green to blue gray-------

Sandstone and siltstone, brown-

Siltstone, blue-gray

Sandstone, well-consolidated-

Coal

Sandstone, coarser than above, wel1-consolidated-------

Siltstone, gray-green; hole yields about $2 \mathrm{gal} / \mathrm{min----}$

Siltstone with layers of fine, poorly consolidated sandst one--

Siltstone, blue-gray; hole yields about $5 \mathrm{gal} / \mathrm{min}----$

We11 SC 5-65- 9ACD. Altitude, 5,770 feet

Clay, silty, soft, dark-brown

Clay, silty, brown; tougher drilling

Clay, silty, yellow-brown; tough drilling

We11 SC 5-65- 9BAA. Altitude, 5,740 feet

Sand, coarse, poorly sorted, light-brown--

Clay, silty; color grading from dark brown to red brown 
Table 3.--Logs of wells drilled by the U.S. Geological Survey--Continued

$\begin{array}{cc}\text { Thick- } & \text { Depth } \\ \text { ness } & \text { (feet) }\end{array}$

We11 SC 5-65- 9DDA. Altitude, 5,810 feet

Sand, coarse, with a little silt--_-_-_-

6

6

Clay, silty, with coarse sand; color grading from

light brown to blue gray--

5

11 
Table 4.--Chemical analyses of sewage sludge and

[Analyses by Metropolitan

\begin{tabular}{|c|c|c|c|c|c|c|c|}
\hline \multirow[b]{3}{*}{ Type } & \multirow{3}{*}{$\begin{array}{l}\text { Specific } \\
\text { conduct- } \\
\text { ance }(\mathrm{mi}- \\
\text { cromhos } / \mathrm{cm})\end{array}$} & \multirow[b]{3}{*}{$\mathrm{pH}$} & \multicolumn{5}{|c|}{ Chemical analyses of sewage } \\
\hline & & & \multicolumn{5}{|c|}{ Concentration, in } \\
\hline & & & $\begin{array}{l}\text { Iron } \\
(\mathrm{Fe})\end{array}$ & $\begin{array}{c}\text { Manga- } \\
\text { nese } \\
(\mathrm{Mn})\end{array}$ & $\begin{array}{l}\text { Ca1- } \\
\text { cium } \\
\text { (Ca) }\end{array}$ & $\begin{array}{l}\text { Ammo- } \\
\text { nia } \\
\left(\mathrm{NH}_{4}\right)\end{array}$ & $\begin{array}{l}\text { Total } \\
\text { Kjeldah1 } \\
\text { nitrogen } \\
\text { (TKN) }\end{array}$ \\
\hline 1 & 5,800 & 7.5 & 10,000 & 200 & 26,000 & 19,000 & 45,000 \\
\hline 2 & 4,800 & 6.3 & 7,300 & 150 & 16,000 & 6,600 & 62,000 \\
\hline 3 & 10,000 & 11.5 & 21,000 & 260 & 67,000 & 1,400 & 38,000 \\
\hline
\end{tabular}

Chemical analyses of water from

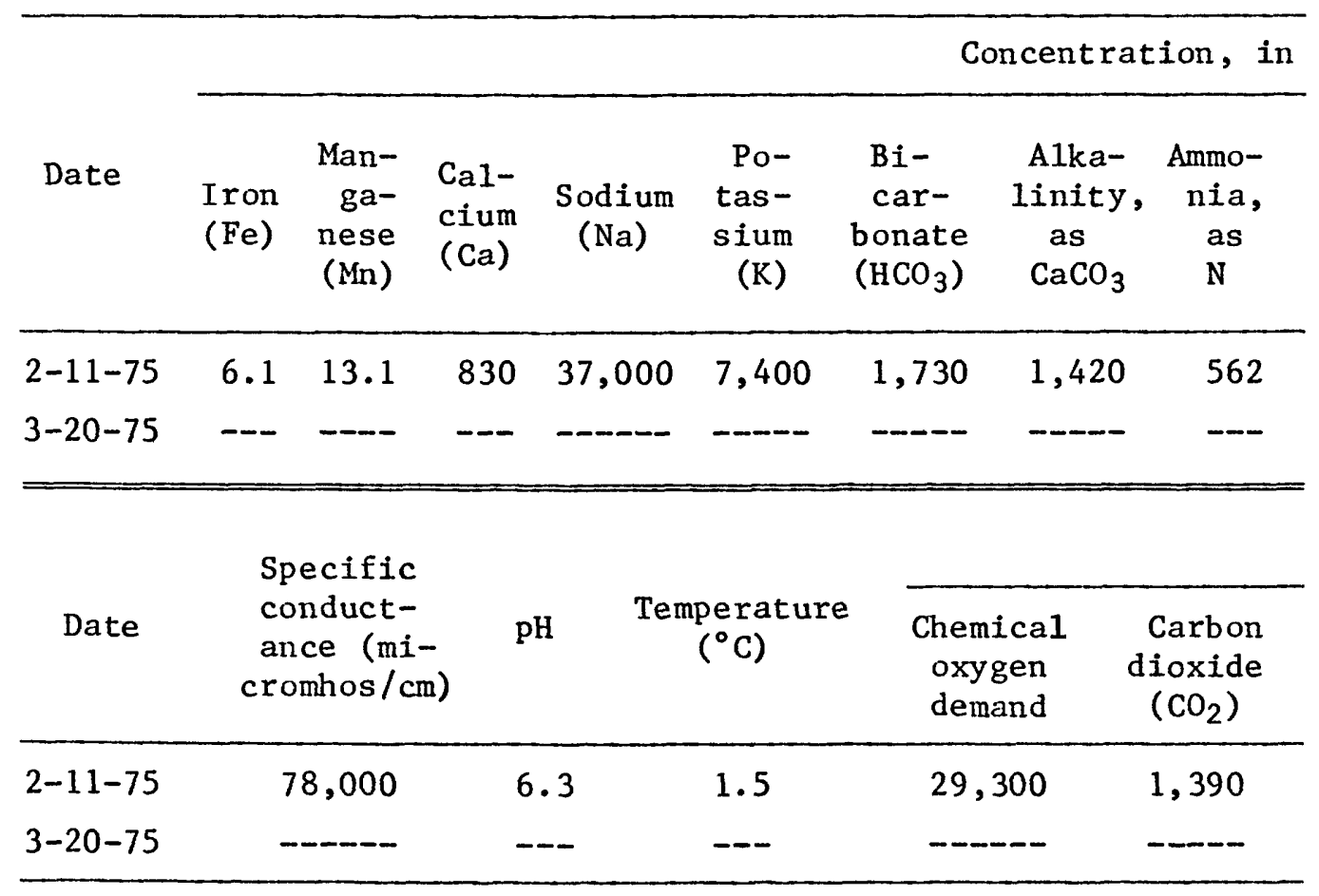


water from the landfill liquid-waste-disposal trench

Denver Sewage Disposal District]

sludge for August and September 1974

parts per million dry weight

\begin{tabular}{cccccccccc}
\hline $\begin{array}{l}\text { Total } \\
\text { phos- } \\
\text { phorus } \\
(\mathrm{P})\end{array}$ & $\begin{array}{c}\text { Cad- } \\
\text { mium }\end{array}$ & $\begin{array}{c}\text { Chro- } \\
\text { mium } \\
(\mathrm{Cr})\end{array}$ & $\begin{array}{c}\text { Copper } \\
\text { (Cu) }\end{array}$ & $\begin{array}{l}\text { Lead } \\
(\mathrm{Pb})\end{array}$ & $\begin{array}{c}\text { Nickel } \\
\text { (Ni) }\end{array}$ & $\begin{array}{l}\text { Zinc } \\
(\mathrm{Zn})\end{array}$ & $\begin{array}{c}\text { Fecal } \\
\text { coliform } \\
\text { (colonies } \\
\text { per gram) }\end{array}$ & $\begin{array}{c}\text { Strep- } \\
\text { tococci } \\
\text { (colonies } \\
\text { per gram) }\end{array}$ \\
\hline 13,000 & 12 & 800 & 810 & 1,100 & 220 & 1,900 & $1.8 \times 10^{4}$ & $5.7 \times 10^{3}$ \\
20,000 & 13 & 990 & 1,100 & 660 & 430 & 3,200 & $5.7 \times 10^{5}$ & $6.8 \times 10^{5}$ \\
20,000 & 9.7 & 630 & 780 & 460 & 320 & 1,200 & $<10$ & $8.9 \times 10^{3}$
\end{tabular}

landfill liquid-waste-disposal trench

milligrams per liter

\begin{tabular}{cccccccc}
\hline $\begin{array}{c}\text { Ammo- } \\
\text { nia, } \\
\text { as } \\
\mathrm{NH}_{4}\end{array}$ & $\begin{array}{c}\text { Dissolved } \\
\text { organic } \\
\text { nitrogen } \\
(\mathrm{N})\end{array}$ & $\begin{array}{c}\text { Kje1- } \\
\text { dah1 } \\
\text { nitro- } \\
\text { gen } \\
(\mathrm{N})\end{array}$ & $\begin{array}{c}\text { Dissolved } \\
\text { orthophos- } \\
\text { phorous } \\
(\mathrm{P})\end{array}$ & $\begin{array}{c}\text { Dissolved } \\
\text { ortho- } \\
\text { phosphate } \\
\left(\mathrm{PO}_{4}\right)\end{array}$ & $\begin{array}{c}\text { Dis- } \\
\text { solved } \\
\text { solids } \\
(\text { resi- } \\
\text { due at } \\
\left.105^{\circ} \mathrm{C}\right)\end{array}$ & $\begin{array}{c}\text { Hard- } \\
\text { ness } \\
\text { (Ca, }\end{array}$ & $\begin{array}{c}\text { Noncar- } \\
\text { bonate } \\
\text { hard- } \\
\text { ness }\end{array}$ \\
\hline 720 & 81 & 643 & 55 & 170 & 20,200 & 2,300 & 880 \\
\hline- & -- & - & -- & - & - & - & - \\
\hline
\end{tabular}

Concentration, in milligrams per liter

\begin{tabular}{ccccccccc}
\hline $\begin{array}{c}\text { Dissolved } \\
\text { organic } \\
\text { carbon }\end{array}$ & Phenols & $\begin{array}{c}\text { Cadmium } \\
(\mathrm{Cd})\end{array}$ & $\begin{array}{c}\text { Chromium } \\
(\mathrm{Cr})\end{array}$ & $\begin{array}{c}\text { Copper } \\
(\mathrm{Cu})\end{array}$ & $\begin{array}{c}\text { Lead } \\
(\mathrm{Pb})\end{array}$ & $\begin{array}{c}\text { Nickel } \\
(\mathrm{Ni})\end{array}$ & $\begin{array}{c}\text { Zinc } \\
(\mathrm{Zn})\end{array}$ \\
\hline 8,100 & 4,660 & 0.032 & 0.25 & 0.030 & 0.19 & 1.28 & 0.25 \\
\hline & 392 & - & - & -- & - & - & - \\
\hline
\end{tabular}


The following table consists of records for 53 we11s. The table format consists of six pages of data for each group of six to nine we1ls. The first page for each group contains the local well number for each we11 in the group. On each of the five subsequent pages for each group, the date of sample is used to identify the wells.

Appropriate headnotes for the table are: Analytical results in milligrams per liter (MG/L) or in micrograms per liter (UG/L), as indicated. Dashes (-) indicate constituent for which no analysis was made. Constituents with concentrations below the detection limit of the analytical procedure are indicated by ND. Code for agency analyzing sample: 9999, Metropolitan Denver Sewage Disposal District; --, U.S. Geological Survey. 
Table 5.--Chemical analyses of

LOCAL IOENT I-

FIER

SC00406519CCC

SC00406528AAB

$5 C 0040652888 C$

SC00406528BDD

$$
\begin{gathered}
\text { DATE } \\
\text { OF } \\
\text { SAMPLE }
\end{gathered}
$$

TOTAL

DEPTH

OF

WELL

(FT)

DIS-
SOLVED
SILICA
(SIOZ)
(MG/L)

OISSOLVED IRON (FE) (UG/L)
SC00406528DCA

SC00406529AAA

SC00406530AAB

SC00406530BDD

sc00406528800

SC00406528DCA

\begin{tabular}{|c|c|c|c|}
\hline $\begin{array}{l}75-05-07 \\
75-08-14 \\
74-10-15\end{array}$ & 635 & $=$ & $\begin{array}{r}280 \\
210 \\
--\end{array}$ \\
\hline $\begin{array}{l}74-11-27 \\
75-05-05 \\
75-08-15 \\
74-11-27 \\
75-08-14\end{array}$ & $\begin{array}{r}635 \\
635 \\
635 \\
21 \\
21\end{array}$ & $\begin{array}{l}-- \\
-- \\
--\end{array}$ & $\begin{array}{l}10 \\
\text { NO } \\
20 \\
10 \\
30\end{array}$ \\
\hline $\begin{array}{l}74-05-01 \\
74-11-27 \\
75-02-11 \\
75-08-15 \\
75-12-08\end{array}$ & $\begin{array}{l}19 \\
19 \\
19 \\
19 \\
19\end{array}$ & $\begin{array}{l}- \\
\therefore \\
\therefore \\
-\end{array}$ & $\begin{array}{l}\text { ND } \\
40 \\
60 \\
20 \\
30\end{array}$ \\
\hline $\begin{array}{l}75-12-08 \\
76-04-05 \\
76-04-16 \\
74-11-27 \\
75-02-12\end{array}$ & $\begin{array}{l}19 \\
19 \\
19 \\
20 \\
20\end{array}$ & $\begin{array}{l}-- \\
-- \\
-- \\
--\end{array}$ & $\begin{array}{l}30 \\
20 \\
40\end{array}$ \\
\hline $\begin{array}{l}75-02-12 \\
75-05-01 \\
75-08-15 \\
75-12-08 \\
75-12-08\end{array}$ & $\begin{array}{l}20 \\
20 \\
20 \\
20 \\
20\end{array}$ & $\begin{array}{r}25 \\
=- \\
=- \\
=-\end{array}$ & $\begin{array}{l}30 \\
\text { ND } \\
30 \\
70 \\
--\end{array}$ \\
\hline $\begin{array}{l}74-10-11 \\
75-05-01 \\
75-08-15 \\
74-10-15 \\
74-11-27\end{array}$ & $\begin{array}{r}33 \\
33 \\
33 \\
573 \\
573\end{array}$ & $\begin{array}{l}-- \\
-- \\
-- \\
--\end{array}$ & $\begin{array}{r}- \\
30 \\
20 \\
-- \\
220\end{array}$ \\
\hline $\begin{array}{l}75-05-05 \\
75-08-15 \\
74-10-15 \\
74-11-27 \\
75-02-13\end{array}$ & $\begin{array}{l}573 \\
573 \\
300 \\
300 \\
300\end{array}$ & $\begin{array}{l}-- \\
-- \\
--\end{array}$ & $\begin{array}{r}40 \\
130 \\
-- \\
\text { ND } \\
30\end{array}$ \\
\hline $\begin{array}{l}75-05-05 \\
75-08-18 \\
75-12-09 \\
75-12-09 \\
74-11-26\end{array}$ & $\begin{array}{r}300 \\
300 \\
300 \\
300 \\
23\end{array}$ & $\begin{array}{l}-- \\
-- \\
-- \\
--\end{array}$ & $\begin{array}{r}360 \\
70 \\
50 \\
-- \\
30\end{array}$ \\
\hline
\end{tabular}

SC00406530BDA

800 
water from wells--Continued

\begin{tabular}{cccccc} 
& \multicolumn{3}{c}{ DIS- } & & DIS- \\
DIS- & DIS- & SOLVED & & SOLVED \\
SOLVED & SOLVED & MAG- & OIS- & PO- & \\
MAN- & CAL- & NE- & SOLVED & TAS- & BICAR- \\
GANESE & CIUM & SIUM & SODIUM & SIUM & BONATE \\
$(M N)$ & (CA) & $(M G)$ & (NA) & $(K)$ & $($ HCO3) \\
$(U G / L)$ & $(M G / L)$ & $(M G / L)$ & $(M G / L)$ & $(M G / L)$ & $(M G / L)$
\end{tabular}

\begin{tabular}{|c|c|c|c|c|c|}
\hline-- & $\begin{array}{l}13 \\
12\end{array}$ & $\because$ & $\begin{array}{l}95 \\
70\end{array}$ & 8.0 & 210 \\
\hline-- & -- & -- & -- & - & - \\
\hline $\begin{array}{l}\text { ND } \\
-- \\
\text { ND } \\
\text { ND } \\
40\end{array}$ & $\begin{array}{c}50 \\
4.8 \\
3.4 \\
195 \\
120\end{array}$ & $\begin{array}{l}-\infty \\
-- \\
-- \\
-\infty\end{array}$ & $\begin{array}{r}70 \\
61 \\
55 \\
145 \\
145\end{array}$ & $\begin{array}{l}3.4 \\
14^{-0} \\
11 \\
18\end{array}$ & $\begin{array}{r}16 n \\
-0 \\
145 \\
336 \\
296\end{array}$ \\
\hline $\begin{array}{l}1400 \\
400 \\
250 \\
280\end{array}$ & $\begin{array}{r}128 \\
115 \\
130 \\
99 \\
200\end{array}$ & $\begin{array}{l}-- \\
-- \\
-- \\
--\end{array}$ & $\begin{array}{r}104 \\
90 \\
88 \\
70 \\
83\end{array}$ & $\begin{array}{l}10^{--} \\
7.4 \\
6.0 \\
8.5\end{array}$ & $\begin{array}{l}-- \\
372 \\
223 \\
263 \\
287\end{array}$ \\
\hline 200 & $137^{--}$ & $\begin{array}{l}26 \\
27\end{array}$ & $78^{--}$ & $10^{-\infty}$ & 304 \\
\hline $\begin{array}{l}210 \\
620\end{array}$ & $217^{-\infty}$ & 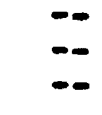 & $\begin{array}{l}142^{-\infty} \\
100\end{array}$ & $11_{4.7}^{--}$ & $\begin{array}{l}439 \\
400\end{array}$ \\
\hline $\begin{array}{r}810 \\
-- \\
290 \\
320\end{array}$ & $\begin{array}{l}230 \\
164 \\
120 \\
205\end{array}$ & 35 & $\begin{array}{r}120 \\
94 \\
110 \\
127\end{array}$ & $\begin{array}{l}5.6 \\
12^{-} \\
10^{-0}\end{array}$ & $\begin{array}{r}501 \\
384 \\
414\end{array}$ \\
\hline-- & - & 35 & - & $\cdots$ & $-\infty$ \\
\hline 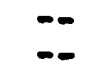 & $202^{--}$ & -- & $124^{--}$ & $-\infty$ & $-\infty$ \\
\hline ND & 130 & -- & 130 & 14 & 307 \\
\hline ND & $130^{-\infty}$ & - & $127^{--}$ & 4.4 & $2 \overrightarrow{3 n}$ \\
\hline 20 & $\begin{array}{l}12 \\
12\end{array}$ & 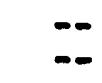 & $\begin{array}{l}80 \\
70\end{array}$ & $13^{-\infty}$ & 228 \\
\hline $\begin{array}{l}-\overline{N D} \\
30\end{array}$ & $128^{--}$ & $=$ & $\begin{array}{l}102^{--} \\
118\end{array}$ & $\begin{array}{l}-- \\
4.7 \\
3.6\end{array}$ & $\begin{array}{l}175 \\
167\end{array}$ \\
\hline $\begin{array}{l}-- \\
50 \\
20 \\
--\end{array}$ & $\begin{array}{l}9.8 \\
28 \\
16\end{array}$ & 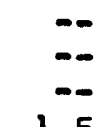 & $\begin{array}{r}95 \\
110 \\
97\end{array}$ & $\begin{array}{l}-\overline{-} \\
8.0 \\
4.3\end{array}$ & $\begin{array}{l}176 \\
174\end{array}$ \\
\hline 500 & $55^{--}$ & 1.5 & $70^{-\infty}$ & 5.0 & $25 \overline{4}$ \\
\hline
\end{tabular}


Table 5.--Chemical analyses of

\begin{tabular}{|c|c|c|c|c|c|}
\hline $\begin{array}{c}\text { OATE } \\
\text { OF } \\
\text { SAMPLE }\end{array}$ & $\begin{array}{l}\text { CAR- } \\
\text { BONATE } \\
\text { (CO3) } \\
\text { (MG/L) }\end{array}$ & $\begin{array}{l}\text { HY- } \\
\text { DROX- } \\
\text { IDE } \\
\text { (OH) } \\
(M G / L)\end{array}$ & $\begin{array}{l}\text { ALKA- } \\
\text { LINITY } \\
\text { AS } \\
\text { CACO3 } \\
\text { (MG/L) }\end{array}$ & $\begin{array}{l}\text { DIS- } \\
\text { SOLVED } \\
\text { SULFATE } \\
\text { (SO4) } \\
\text { (MG/L) }\end{array}$ & $\begin{array}{l}\text { DIS- } \\
\text { SOLVED } \\
\text { CHLO- } \\
\text { RIUE } \\
\text { (CL) } \\
(M G / L)\end{array}$ \\
\hline
\end{tabular}

75-05-07

$75-08-14$

$74-10-15$

$-$

NO

172

--

ND

8.0

$74-11-27$

75-05-05

$75-08-15$

$74-11-27$

$75-08-14$

--

131

ND

$-$

$-$

ND

119

ND

ND

$--$

276

NO

243

74-05-01

$74-11-27$

$75-02-11$

$75-08-15$

$75-12-08$

$-\infty$

$-\infty$

305

183

--

ND

--

ND

216

ND

235

--

7.0

$75-12-08$

$76-04-05$

76-04-16

$74-11-27$

75-02-12

75-02-12

75-0s-01

$75-08-15$

$75-12-08$

$75-12-08$

74-10-11

75-05-01

75-08-15

74-10-15

$74-11-27$

\section{ND}

NO

NO

ND

249

$\infty$

--

360

328

$-$

411

ND

ND

-

--

315

340

ND

--

$-$

ND

$--$

No

ND

252

75-05-05

75-08-15

74-10-15

$74-11-27$

75-02-13

ND

NO

187

-

NO

144

137

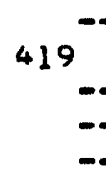

$-$

1.6

ND

820

$-$

4.0

4.0

76

\begin{tabular}{ll}
$\cdots$ & 64 \\
375 & 40 \\
420 & 34 \\
\hline- & 45
\end{tabular}

75-05-05

75-08-18

75-12-09

$75-12-09$

74-11-26

-

$-$

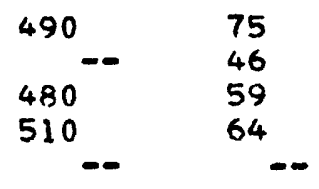

ND

ND

--
NO
ND
--

144
143
208

$\begin{array}{cc}\ldots & 84 \\ 480 & 98 \\ \ldots & 49 \\ \ldots & 4.2 \\ \ldots .2 \\ \ldots & 7.0 \\ \ldots & 7.0 \\ \ldots & 63 \\ \ldots & 62 \\ \ldots & 66 \\ 32 & 75 \\ 23 & 64 \\ \ldots & 68 \\ \ldots & 4.8\end{array}$


water from wells--Continued

OIS-

SOLVED

FLUO-

RIDE

DIS-

SOLVED

DIS_ DIS-

SOLVEO

NITRATE

TOTAL SOLVED

NITRITE

(F)

(N)

NITRATE NITRATE

$\begin{array}{lllll}(N 03) & \text { (NO3) (N) } & \text { (NO2) } & \text { (NO2) }\end{array}$

$\begin{array}{lllll}(N 03) & \text { (NO3) } & \text { (N) } & \text { (NO2) } & \text { (NO2) }\end{array}$

$(M G / L) \quad(M G / L) \quad(M G / L) \quad(M G / L) \quad(M G / L) \quad(M G / L) \quad(M G / L)$

DIS

TOTAL SOLVEO

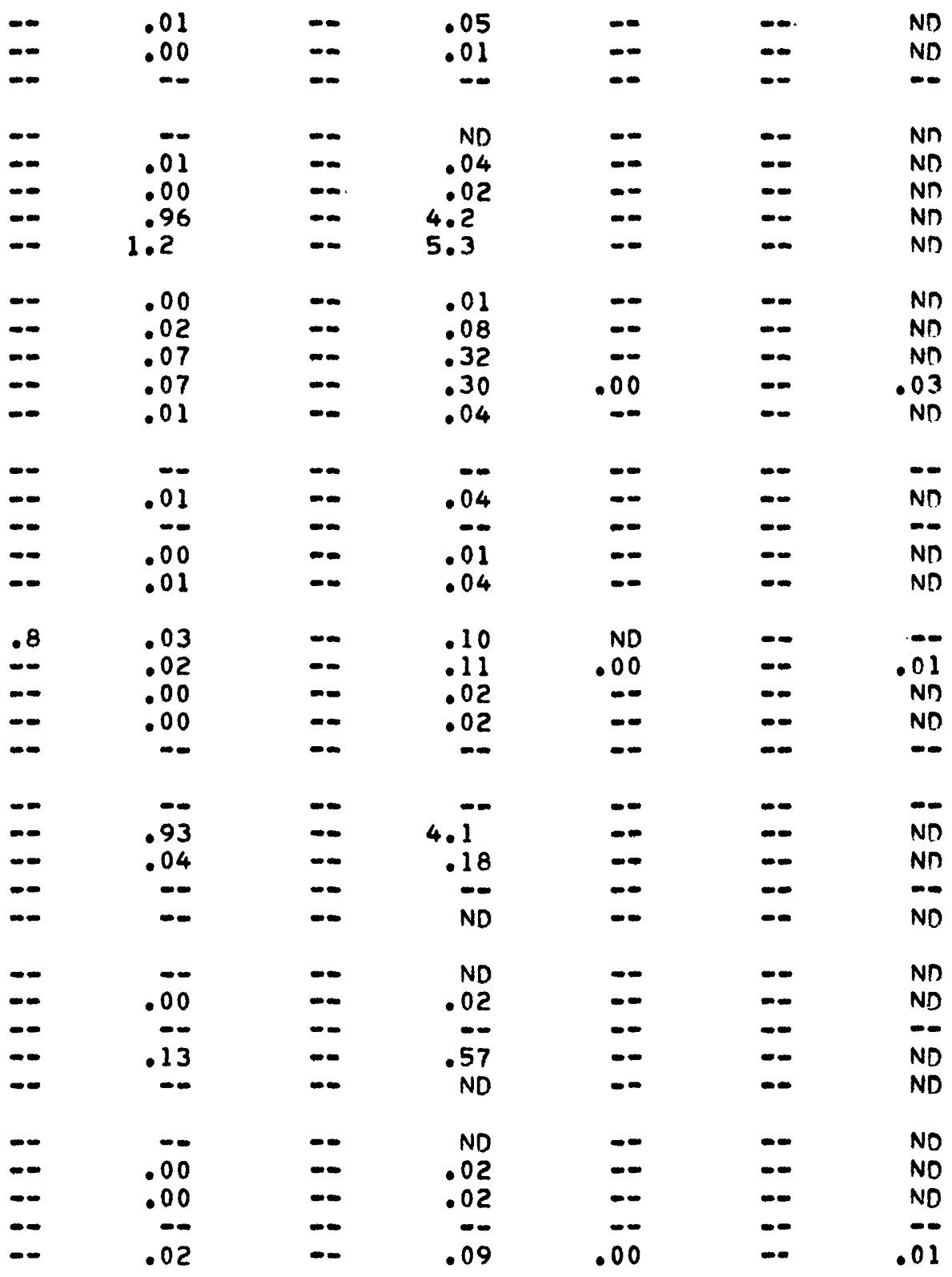




\begin{tabular}{|c|c|c|c|c|c|}
\hline & $\begin{array}{l}\text { DIS- } \\
\text { SOLVED } \\
\text { AMMONIA }\end{array}$ & & DIS & $\begin{array}{l}\text { DIS- } \\
\text { SOLVED } \\
\text { ORGANIC }\end{array}$ & $\begin{array}{l}\text { DIS- } \\
\text { SOLVED } \\
\text { KJEL. }\end{array}$ \\
\hline $\begin{array}{c}\text { DATE } \\
\text { OF } \\
\text { SAMPLE }\end{array}$ & $\begin{array}{l}\text { NITRO- } \\
\text { GEN } \\
\text { (N) } \\
(M G / L)\end{array}$ & $\begin{array}{l}\text { TOTAL } \\
\text { AMMONIA } \\
\text { (NH4) } \\
\text { (MG/L) }\end{array}$ & $\begin{array}{l}\text { SOLVED } \\
\text { AMMONIA } \\
\text { (NH4) } \\
\text { (MG/L) }\end{array}$ & $\begin{array}{c}\text { NITRO- } \\
\text { GEN } \\
\text { (N) } \\
(M G / L)\end{array}$ & $\begin{array}{c}\text { NI TRO- } \\
\text { GEN } \\
\text { (N) } \\
(M G / L)\end{array}$ \\
\hline
\end{tabular}

\begin{tabular}{|c|c|c|c|c|c|}
\hline $\begin{array}{l}75-05-07 \\
75-08-14 \\
74-10-15\end{array}$ & $\begin{array}{r}.20 \\
.20 \\
--\end{array}$ & $=$ & $\begin{array}{r}.26 \\
.26 \\
--\end{array}$ & $\begin{array}{l}-- \\
--\end{array}$ & $\begin{array}{l}\text { NO } \\
\text { ND } \\
--\end{array}$ \\
\hline $\begin{array}{l}74-11-27 \\
75-05-05 \\
75-08-15 \\
74-11-27 \\
75-08-14\end{array}$ & $\begin{array}{l}\text { ND } \\
.20 \\
.20 \\
\text { ND } \\
\text { ND }\end{array}$ & $\begin{array}{l}-- \\
-- \\
--\end{array}$ & $\begin{array}{l}-- \\
.26 \\
.26 \\
-- \\
--\end{array}$ & $\begin{array}{l}= \\
-- \\
-\infty\end{array}$ & $\begin{array}{r}.40 \\
\text { ND } \\
.10 \\
.20 \\
.30\end{array}$ \\
\hline $\begin{array}{l}74-05-01 \\
74-11-27 \\
75-02-11 \\
75-08-15 \\
75-12-08\end{array}$ & $\begin{array}{l}\text { ND } \\
\text { ND } \\
\text { ND } \\
.10 \\
\text { ND }\end{array}$ & $\begin{array}{l}-\infty \\
=- \\
=- \\
=\end{array}$ & 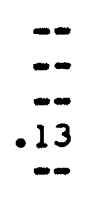 & $\because=$ & $\begin{array}{r}.30 \\
4.2 \\
.80 \\
.20 \\
.10\end{array}$ \\
\hline $\begin{array}{l}75-12-08 \\
76-04-05 \\
76-04-16 \\
74-11-27 \\
75-02-12\end{array}$ & $\begin{array}{l}-- \\
\text { ND } \\
-- \\
\text { ND } \\
\text { ND }\end{array}$ & $\begin{array}{l}-- \\
-- \\
-- \\
--\end{array}$ & $\begin{array}{l}-- \\
-- \\
-- \\
--\end{array}$ & $\begin{array}{l}= \\
=- \\
=- \\
--\end{array}$ & $\begin{array}{r}.40 \\
.40 \\
3.0 \\
.80\end{array}$ \\
\hline $\begin{array}{l}75-02-12 \\
75-05-01 \\
75-08-15 \\
75-12-08 \\
75-12-08\end{array}$ & $\begin{array}{r}.01 \\
\text { ND } \\
.40 \\
\text { ND } \\
--\end{array}$ & $\begin{array}{l}-- \\
=- \\
-- \\
--\end{array}$ & $\begin{array}{r}.01 \\
.52 \\
-. \\
--\end{array}$ & $\begin{array}{l}.34 \\
-- \\
-- \\
--\end{array}$ & $\begin{array}{r}.35 \\
.30 \\
.30 \\
.20 \\
--\end{array}$ \\
\hline $\begin{array}{l}74-10-11 \\
75-05-01 \\
75-08-15 \\
74-10-15 \\
74-11-27\end{array}$ & $\begin{array}{r}-- \\
\text { NO } \\
.20 \\
-- \\
\text { NO }\end{array}$ & $\begin{array}{l}-- \\
-- \\
--\end{array}$ & $\begin{array}{l}=- \\
-26 \\
-- \\
--\end{array}$ & $\begin{array}{l}-- \\
10 \\
--\end{array}$ & $\begin{array}{r}-- \\
.30 \\
.06 \\
-0\end{array}$ \\
\hline $\begin{array}{l}75-05-05 \\
75-08-15 \\
74-10-15 \\
74-11-27 \\
75-02-13\end{array}$ & $\begin{array}{l}.20 \\
.30 \\
-- \\
\text { ND } \\
\text { ND }\end{array}$ & $\begin{array}{l}-- \\
-- \\
-- \\
--\end{array}$ & $\begin{array}{l}.26 \\
.39 \\
-- \\
-- \\
--\end{array}$ & $\begin{array}{l}.0 \\
10 \\
-- \\
--\end{array}$ & $\begin{array}{r}.10 \\
.40 \\
-. \\
.90 \\
.30\end{array}$ \\
\hline $\begin{array}{l}75-05-05 \\
75-08-18 \\
75-12-09 \\
75-12-09 \\
74-11-26\end{array}$ & $\begin{array}{l}.30 \\
\text { ND } \\
\text { ND } \\
-- \\
\text { ND }\end{array}$ & $\begin{array}{l}-- \\
-- \\
--\end{array}$ & $\begin{array}{l}.39 \\
-- \\
-- \\
--\end{array}$ & $\begin{array}{l}-- \\
-- \\
-- \\
-\end{array}$ & $\begin{array}{r}\text { ND } \\
.30 \\
.20 \\
.30\end{array}$ \\
\hline
\end{tabular}


water from welzs--Continued

$\begin{array}{lllllll}\text { DIS- } & \text { OIS- } & \text { DIS- } & \text { DIS- } & \text { DIS- } & & \\ \text { SOLVED } & \text { SOLVED } & \text { SOLVED } & \text { SOLVED } & \text { SOLVED } & & \text { NON- } \\ \text { ORTHO. } & \text { ORTHO } & \text { SOLIDS } & \text { SOLIDS } & \text { SOLIDS } & & \text { CAR- } \\ \text { PHOS- } & \text { PHOS- } & \text { (RESI- } & \text { (RESI- } & \text { (SUM OF } & \text { HARO- } & \text { BONATF } \\ \text { PHORUS } & \text { PHATE } & \text { DUE AT } & \text { DUE AT } & \text { CONSTI- } & \text { NESS } & \text { HARD- } \\ (P) & (P O 4) & \left.180^{\circ} C\right) & \left.105^{\circ} C\right) & \text { TUENTS) } & \text { (CA,MG) } & \text { NESS } \\ (M G / L) & (M G / L) & (M G / L) & (M G / L) & (M G / L) & (M G / L) & \text { (MG/L) }\end{array}$

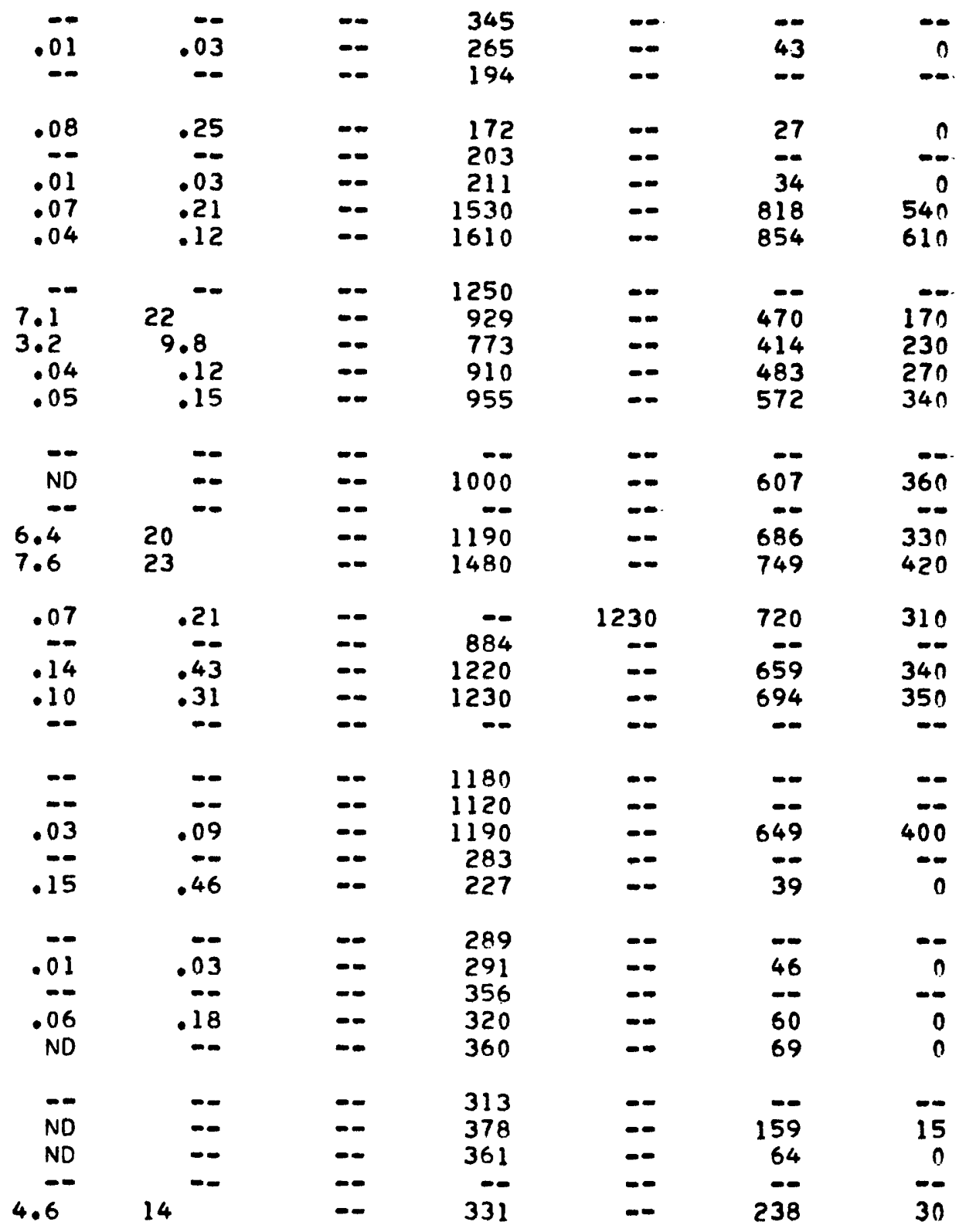




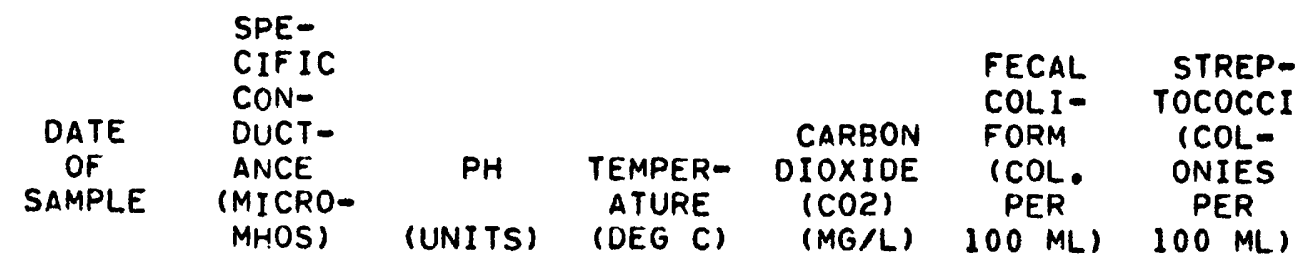

\begin{tabular}{|c|c|c|c|c|c|c|}
\hline $\begin{array}{l}75-05-07 \\
75-08-14 \\
74-10-15\end{array}$ & $\begin{array}{r}460 \\
480 \\
--\end{array}$ & $\begin{array}{c}8.3 \\
8.1 \\
--\end{array}$ & $\begin{array}{r}16.5 \\
17.5 \\
--\end{array}$ & 2.7 & $-\infty$ & $\begin{array}{l}-\infty \\
-\infty\end{array}$ \\
\hline $\begin{array}{l}74-11-27 \\
75-05-05 \\
75-08-15 \\
74-11-27 \\
75-08-14\end{array}$ & $\begin{array}{r}310 \\
300 \\
340 \\
2000 \\
2400\end{array}$ & $\begin{array}{l}8.2 \\
8.4 \\
8.7 \\
7.0 \\
7.2\end{array}$ & $\begin{array}{r}14.5 \\
18.0 \\
16.5 \\
9.0 \\
14.5\end{array}$ & $\begin{array}{l}1.4 \\
54 \\
30 \\
.5\end{array}$ & $\begin{array}{l}-\infty \\
-\infty \\
-\infty\end{array}$ & $\begin{array}{l}-\infty \\
-\infty \\
-\infty \\
-\infty\end{array}$ \\
\hline $\begin{array}{l}74-05-01 \\
74-11-27 \\
75-02-11 \\
75-08-15 \\
75-12-08\end{array}$ & $\begin{array}{l}1300 \\
1350 \\
1100 \\
1400 \\
1550\end{array}$ & $\begin{array}{l}7.3 \\
7.0 \\
7.2 \\
7.6 \\
8.3\end{array}$ & $\begin{array}{l}10.0 \\
12.5 \\
10.5 \\
13.5 \\
11.0\end{array}$ & $\begin{array}{l}60^{--} \\
23 \\
11 \\
2.3\end{array}$ & $\begin{array}{l}-- \\
-\infty \\
-\infty\end{array}$ & $\begin{array}{r}-\infty \\
-- \\
-\infty\end{array}$ \\
\hline $\begin{array}{l}75-12-08 \\
76-04-05 \\
76-04-16 \\
74-11-27 \\
75-02-12\end{array}$ & $\begin{array}{r}1550 \\
1300 \\
1700 \\
1900\end{array}$ & $\begin{array}{r}8.3 \\
7.5 \\
-.0 \\
6.9 \\
6.9\end{array}$ & $\begin{array}{r}11.0 \\
11.5 \\
-0 \\
13.5 \\
14.0\end{array}$ & $\begin{array}{l}15^{--} \\
88^{--} \\
81^{--}\end{array}$ & $\begin{array}{l}-- \\
-- \\
--\end{array}$ & $\begin{array}{r}-\infty \\
-\infty \\
-\infty\end{array}$ \\
\hline $\begin{array}{l}75-02-12 \\
75-05-01 \\
75-08-15 \\
75-12-08 \\
75-12-08\end{array}$ & $\begin{array}{l}1900 \\
1950 \\
1950 \\
1950 \\
1950\end{array}$ & $\begin{array}{l}6.9 \\
6.8 \\
7.7 \\
8.1 \\
8.1\end{array}$ & $\begin{array}{l}14.0 \\
10.0 \\
12.5 \\
13.0 \\
13.0\end{array}$ & $\begin{array}{c}101^{12} \\
5.3 \\
-\infty\end{array}$ & $\begin{array}{l}-- \\
\ddot{-} \\
-\end{array}$ & $\begin{array}{l}-\infty \\
-\infty \\
-\infty\end{array}$ \\
\hline $\begin{array}{l}74-10-11 \\
75-05-01 \\
75-08-15 \\
74-10-15 \\
74-11-27\end{array}$ & $\begin{array}{r}-- \\
1950 \\
1800 \\
-- \\
440\end{array}$ & $\begin{array}{r}-- \\
6.8 \\
7.5 \\
-- \\
7.9\end{array}$ & $\begin{array}{r}-0 \\
10.0 \\
16.0 \\
14.0\end{array}$ & $16^{--}$ & $\begin{array}{l}-- \\
-- \\
--\end{array}$ & $\begin{array}{l}-\infty \\
-\infty \\
-\infty \\
-\infty\end{array}$ \\
\hline $\begin{array}{l}75-05-05 \\
75-08-15 \\
74-10-15 \\
74-11-27 \\
75-02-13\end{array}$ & $\begin{array}{l}480 \\
500 \\
-- \\
600 \\
640\end{array}$ & $\begin{array}{r}8.1 \\
8.4 \\
-1.9 \\
7.9 \\
7.9\end{array}$ & $\begin{array}{r}17.5 \\
19.0 \\
-0 \\
11.5 \\
13.0\end{array}$ & $\begin{array}{r}-.-5 \\
1.5 \\
--5 \\
3.5 \\
3.4\end{array}$ & $\begin{array}{l}-- \\
-- \\
0\end{array}$ & $\begin{array}{l}-\infty \\
-- \\
-- \\
0\end{array}$ \\
\hline $\begin{array}{l}75-05-05 \\
75-08-18 \\
75-12-09 \\
75-12-09 \\
74-11-26\end{array}$ & $\begin{array}{l}590 \\
675 \\
725 \\
725 \\
600\end{array}$ & $\begin{array}{l}8.5 \\
8.3 \\
8.4 \\
8.4 \\
7.4\end{array}$ & $\begin{array}{l}12.0 \\
14.5 \\
13.0 \\
13.0 \\
11.5\end{array}$ & $\begin{array}{l}-- \\
1.4 \\
1.1 \\
14^{--}\end{array}$ & $\begin{array}{l}-- \\
-- \\
--\end{array}$ & $\begin{array}{l}= \\
-\infty \\
-\infty\end{array}$ \\
\hline
\end{tabular}


water from weIzs--Continued

$\begin{array}{cccccc}\text { CYANIDE } & \text { PHENOLS } & \text { TOTAL } & \text { TOTAL } \\ (C N) & \text { CHLOR- } & \text { TOTAL } & \text { TOTAL } \\ (M G / L) & (U G / L) & \text { ALDRIN } & \text { DANE } & \text { DDO } & \text { DDE } \\ (U G / L) & (U G / L) & (U G / L) & (U G / L)\end{array}$

\begin{tabular}{|c|c|c|c|c|c|}
\hline$-\infty$ & $-\infty$ & -- & $-\infty$ & $-\infty$ & - \\
\hline$-\infty$ & - & $-\infty$ & $-\infty$ & $-\infty$ & $-\infty$ \\
\hline-- & $-\infty$ & $-\infty$ & $-\infty$ & - & $-\infty$ \\
\hline$-\infty$ & - & - & - & - & - \\
\hline - & $-\infty$ & -- & -- & - & $\infty$ \\
\hline-- & 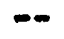 & $=$ & $-\infty$ & $\cdots$ & - \\
\hline - & 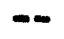 & $=-$ & 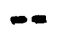 & $\rightarrow$ & - \\
\hline- & $-\infty$ & ND & ND & ND & ND \\
\hline- & $-\infty$ & - & -- & - & $\infty$ \\
\hline- & $-\infty$ & -- & $-\infty$ & - & - \\
\hline & - & $\cdots$ & -- & $\cdots$ & $-\infty$ \\
\hline & & - & -- & $\cdots$ & \\
\hline - & - & $-\infty$ & -- & $\cdots$ & \\
\hline- & $-\infty$ & $-\infty$ & 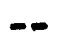 & $-\infty$ & 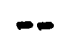 \\
\hline & $-\infty$ & -- & $-\infty$ & - & \\
\hline & $=$ & -- & $\rightarrow$ & -- & \\
\hline & -- & -- & $\rightarrow$ & $-\infty$ & \\
\hline 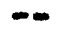 & $-\infty$ & $\cdots$ & $-\infty$ & $-\infty$ & \\
\hline 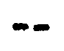 & $\infty$ & - & $-\infty$ & $\rightarrow$ & \\
\hline & -- & $-\infty$ & $m$ & -- & \\
\hline & $-\infty$ & $-\infty$ & -- & $-\infty$ & \\
\hline & -- & $\infty$ & -- & $\ldots$ & \\
\hline & - & $-\infty$ & -- & - & \\
\hline & - & $-\infty$ & $-\infty$ & $m$ & \\
\hline & - & -- & $-\infty$ & $\cdots$ & \\
\hline & -- & $\cdots$ & $-\infty$ & $\cdots$ & \\
\hline & $-\infty$ & - & - & - & \\
\hline & $\cdots$ & $-\infty$ & -- & $\cdots$ & \\
\hline & $-\infty$ & $-\infty$ & -- & $-\infty$ & \\
\hline & $\cdots$ & -- & -- & $-\infty$ & \\
\hline & $-\infty$ & $=$ & - & 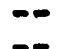 & \\
\hline & $\ldots$ & $-\infty$ & - & $\ldots$ & \\
\hline - & $\ldots$ & -- & $-\infty$ & -- & \\
\hline & $\ldots$ & ND & ND & ND & $r v$ \\
\hline & $\cdots$ & - & -- & -- & \\
\hline & $-\infty$ & -- & $-\infty$ & -- & \\
\hline & $\cdots$ & -- & $-\infty$ & $\cdots$ & \\
\hline
\end{tabular}


Table 5.--Chemical analyses of

\begin{tabular}{|c|c|c|c|c|c|c|}
\hline $\begin{array}{c}\text { DATE } \\
\text { OF } \\
\text { SAMPLE }\end{array}$ & $\begin{array}{l}\text { TOTAL } \\
\text { DDT } \\
\text { (UG/L) }\end{array}$ & $\begin{array}{l}\text { TOTAL } \\
\text { DI- } \\
\text { AZINON } \\
\text { (UG/L) }\end{array}$ & $\begin{array}{l}\text { TOTAL } \\
\text { OI- } \\
\text { ELDRIN } \\
\text { (UG/L) }\end{array}$ & $\begin{array}{l}\text { TOTAL } \\
\text { ENDRIN } \\
\text { (UG/L) }\end{array}$ & $\begin{array}{l}\text { TOTAL } \\
\text { HEPTA- } \\
\text { CHLOR } \\
\text { (UG/L) }\end{array}$ & $\begin{array}{l}\text { HEPTA- } \\
\text { CHLOR } \\
\text { EPOXIDE } \\
\text { (UG/L) }\end{array}$ \\
\hline
\end{tabular}

\begin{tabular}{|c|c|c|c|c|c|c|}
\hline $\begin{array}{l}75-05-07 \\
75-08-14 \\
74-10-15\end{array}$ & $=$ & $=$ & $=$ & $=$ & $=$ & $=$ \\
\hline $\begin{array}{l}74-11-27 \\
75-05-05 \\
75-08-15 \\
74-11-27 \\
75-08-14\end{array}$ & $\begin{array}{l}-- \\
=- \\
=- \\
\text { NO }\end{array}$ & $\begin{array}{l}-- \\
-- \\
\text { ND }\end{array}$ & $\begin{array}{l}-\infty \\
-\infty \\
-\infty \\
\text { ND }\end{array}$ & $\begin{array}{l}= \\
=- \\
=- \\
\text { NO }\end{array}$ & $\begin{array}{l}-\infty \\
-\infty \\
-\infty \\
\text { NO }\end{array}$ & $\begin{array}{l}-- \\
-- \\
-\infty \\
\text { ND }\end{array}$ \\
\hline $\begin{array}{l}74-05-01 \\
74 \div 11-27 \\
75-02-11 \\
75-08-15 \\
75-12-08\end{array}$ & $\begin{array}{l}-- \\
-- \\
-- \\
--\end{array}$ & $\begin{array}{l}-\infty \\
-\infty \\
-\infty \\
-\infty\end{array}$ & $\begin{array}{l}-- \\
-- \\
-- \\
--\end{array}$ & $\begin{array}{l}-- \\
-- \\
-- \\
--\end{array}$ & $\begin{array}{l}-\infty \\
-- \\
-- \\
--\end{array}$ & $\begin{array}{l}- \\
-\infty \\
-\infty\end{array}$ \\
\hline $\begin{array}{l}75-12-08 \\
76-04-05 \\
76-04-16 \\
74-11-27 \\
75-02-12\end{array}$ & $\begin{array}{l}-- \\
-- \\
-- \\
--\end{array}$ & $\begin{array}{l}-- \\
-- \\
--\end{array}$ & $\begin{array}{l}-\infty \\
-\infty \\
-\infty \\
-\infty\end{array}$ & $\begin{array}{l}=- \\
=- \\
=- \\
=-\end{array}$ & $\begin{array}{l}=- \\
-- \\
-- \\
--\end{array}$ & $\begin{array}{l}-\infty \\
-\infty \\
-\infty\end{array}$ \\
\hline $\begin{array}{l}75-02-12 \\
75-05-01 \\
75-08-15 \\
75-12-08 \\
75-12-08\end{array}$ & $\begin{array}{l}-- \\
-- \\
-- \\
--\end{array}$ & $\begin{array}{l}-- \\
-\infty \\
-\infty\end{array}$ & $\begin{array}{l}-- \\
-- \\
-- \\
--\end{array}$ & $\begin{array}{l}-- \\
-- \\
--\end{array}$ & $\begin{array}{l}=- \\
-- \\
-\end{array}$ & 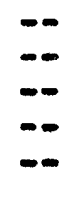 \\
\hline $\begin{array}{l}74-10-11 \\
75-05-01 \\
75-08-15 \\
74-10-15 \\
74-11-27\end{array}$ & $\begin{array}{l}-- \\
-- \\
-- \\
--\end{array}$ & $\begin{array}{l}=- \\
-- \\
-- \\
--\end{array}$ & $\begin{array}{l}-\infty \\
-\infty \\
-\infty \\
-\infty\end{array}$ & $\begin{array}{l}=- \\
=- \\
=- \\
--\end{array}$ & $\begin{array}{l}-\infty \\
-\infty \\
-\infty \\
-\infty\end{array}$ & 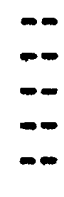 \\
\hline $\begin{array}{l}75-05-05 \\
75-08-15 \\
74-10-15 \\
74-11-27 \\
75-02-13\end{array}$ & $\begin{array}{l}-- \\
-- \\
-- \\
--\end{array}$ & $\begin{array}{l}=- \\
-- \\
-- \\
--\end{array}$ & $\begin{array}{l}-- \\
-- \\
-- \\
--\end{array}$ & $\begin{array}{l}-- \\
-- \\
-\infty \\
--\end{array}$ & $\begin{array}{l}-\infty \\
-- \\
-- \\
--\end{array}$ & - \\
\hline $\begin{array}{l}75-05-05 \\
75-08-18 \\
75-12-09 \\
75-12-09 \\
74-11-26\end{array}$ & $\begin{array}{l}-- \\
\text { NO } \\
-- \\
--\end{array}$ & $\begin{array}{l}-- \\
\text { NO } \\
-- \\
--\end{array}$ & $\begin{array}{l}-- \\
\text { NO } \\
--\end{array}$ & $\begin{array}{l}-- \\
-- \\
--\end{array}$ & $\begin{array}{l}-- \\
N D \\
-- \\
--\end{array}$ & $\begin{array}{l}-- \\
-- \\
--\end{array}$ \\
\hline
\end{tabular}


water from wezls--Continued

\begin{tabular}{|c|c|c|c|c|}
\hline $\begin{array}{l}\text { TOTAL } \\
\text { LINDANE } \\
\text { (UG/L) }\end{array}$ & $\begin{array}{l}\text { TOTAL } \\
\text { MALA- } \\
\text { THION } \\
\text { (UG/L) }\end{array}$ & $\begin{array}{l}\text { TOTAL } \\
\text { METHYL } \\
\text { PARA- } \\
\text { THION } \\
\text { (UG/L) }\end{array}$ & $\begin{array}{l}\text { TOTAL } \\
\text { PARA- } \\
\text { THION } \\
\text { (UG/L) }\end{array}$ & $\begin{array}{l}\text { TOTAL } \\
\text { PCB } \\
\text { (UG/L) }\end{array}$ \\
\hline
\end{tabular}

\begin{tabular}{|c|c|c|c|c|c|}
\hline$-\infty$ & $-\infty$ & $\infty$ & $-\infty$ & $-\infty$ & $-\infty$ \\
\hline$-\infty$ & $\infty$ & $\infty$ & $-\infty$ & $\infty$ & $-\infty$ \\
\hline$-\infty$ & $\cdots$ & -- & $\infty$ & $\cdots$ & $\infty$ \\
\hline$-\infty$ & $-\infty$ & $\infty$ & $-\infty$ & $-\infty$ & - \\
\hline$\infty$ & $-\infty$ & $-\infty$ & $\infty$ & $\infty$ & - \\
\hline$\infty$ & $\infty$ & $\cdots$ & $\Rightarrow$ & $-\infty$ & - \\
\hline-- & $-\infty$ & $-\infty$ & $-\infty$ & $\cdots$ & 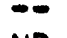 \\
\hline ND & ND & ND & ND & ND & \\
\hline$-\infty$ & $\infty$ & $-\infty$ & $\cdots$ & -- & -- \\
\hline-- & $\infty$ & $-\infty$ & $-\infty$ & $-\infty$ & " \\
\hline$-\infty$ & $\infty$ & $\infty$ & $-\infty$ & $-\infty$ & " \\
\hline$-\infty$ & $-\infty$ & $=$ & $-\infty$ & 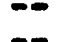 & 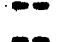 \\
\hline- & $-\infty$ & - & $-\infty$ & - & $\infty$ \\
\hline$-\infty$ & $-\infty$ & $\infty$ & $-\infty$ & $-\infty$ & - \\
\hline$\infty$ & $-\infty$ & $-\infty$ & $-\infty$ & $-\infty$ & \\
\hline$\infty$ & $\infty$ & $-\infty$ & $\cdots$ & $-\infty$ & \\
\hline$\infty$ & $-\infty$ & $\infty$ & $\infty$ & $\infty$ & \\
\hline$-\infty$ & $-\infty$ & $-\infty$ & $-\infty$ & $-\infty$ & \\
\hline$-\infty$ & -- & - & - & $\infty$ & \\
\hline$-\infty$ & $\infty$ & $-\infty$ & $-\infty$ & $-\infty$ & \\
\hline$-\infty$ & $-\infty$ & -- & - & $-\infty$ & \\
\hline$-\infty$ & -- & $-\infty$ & $-\infty$ & $\cdots$ & \\
\hline 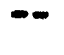 & $-\infty$ & - & $-\infty$ & $-\infty$ & \\
\hline$-\infty$ & -- & $\infty$ & $\infty$ & $\cdots$ & \\
\hline 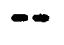 & $-\infty$ & $-\infty$ & $\infty$ & $-\infty$ & \\
\hline • & $\infty$ & $-\infty$ & $-\infty$ & $\infty$ & \\
\hline- & $\infty$ & $\infty$ & $\infty$ & $\infty$ & \\
\hline$-\infty$ & $\infty$ & $\infty$ & $-\infty$ & -- & \\
\hline • & $\infty$ & - & $\infty$ & $-\infty$ & \\
\hline & $\infty$ & $-\infty$ & -- & $\infty$ & \\
\hline - & $-\infty$ & $\infty$ & $-\infty$ & $-\infty$ & \\
\hline & $\cdots$ & $\infty$ & $\cdots$ & $\infty$ & \\
\hline$-\infty$ & $\infty$ & $-\infty$ & $\infty$ & $\infty$ & \\
\hline 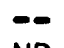 & -- & -- & -- & -- & - \\
\hline ND & ND & ND & ND & ND & dD \\
\hline- & $-\infty$ & $-\infty$ & $\infty$ & $-\infty$ & \\
\hline- & $\infty$ & $=$ & $-\infty$ & $=$ & \\
\hline
\end{tabular}


Table 5.--Chemical analyses of

\begin{tabular}{ccccc}
\multicolumn{7}{c}{} & DIS- & DIS- & & \\
& SOLVED & SOLVED & DIS- & DIS- \\
DATE & CAD- & CHRO- & SOLVED & SOLVED \\
OF & MIUM & MIUM & COPPER & LEAD \\
SAMPLE & $(C D)$ & $(C R)$ & $(C U)$ & $(P B)$ \\
& $(U G / L)$ & $(U G / L)$ & $(U G / L)$ & $(U G / L)$
\end{tabular}

\begin{tabular}{|c|c|c|c|c|}
\hline $\begin{array}{l}75-05-07 \\
75-08-14 \\
74-10-15\end{array}$ & NO & $\overline{\text { ND }}$ & 20 & 20 \\
\hline $\begin{array}{l}74-11-27 \\
75-05-05 \\
75-08-15 \\
74-11-27 \\
75-08-14\end{array}$ & $\begin{array}{r}\text { NO } \\
\text { NO } \\
1 \\
\text { NO }\end{array}$ & $\begin{array}{l}\text { ND } \\
-- \\
\text { ND } \\
30 \\
40\end{array}$ & $\begin{array}{l}\text { ND } \\
-- \\
50 \\
10 \\
20\end{array}$ & $\begin{array}{l}30 \\
-- \\
N D \\
30 \\
10\end{array}$ \\
\hline $\begin{array}{l}74-05-01 \\
74-11-27 \\
75-02-11 \\
75-08-15 \\
75-12-08\end{array}$ & $\begin{array}{r}-- \\
\text { ND } \\
2 \\
\text { ND } \\
5\end{array}$ & $\begin{array}{l}-- \\
\text { ND } \\
20 \\
40 \\
\text { ND }\end{array}$ & $\begin{array}{r}-- \\
\text { ND } \\
10 \\
620 \\
\text { ND }\end{array}$ & $\begin{array}{l}-- \\
10 \\
20 \\
40 \\
40\end{array}$ \\
\hline $\begin{array}{l}75-12-08 \\
76-04-05 \\
76-04-16 \\
74-11-27 \\
75-02-12\end{array}$ & $\begin{array}{l}-- \\
-\infty \\
\text { NO } \\
\text { NO }\end{array}$ & $\begin{array}{l}-- \\
\text { ND } \\
-- \\
10 \\
\text { ND }\end{array}$ & $\begin{array}{l}-- \\
10 \\
-- \\
10\end{array}$ & $\begin{array}{l}-0 \\
20 \\
\text { ND } \\
30\end{array}$ \\
\hline $\begin{array}{l}75-02-12 \\
75-05-01 \\
75-08-15 \\
75-12-08 \\
75-12-08\end{array}$ & $\begin{array}{r}-- \\
-- \\
\text { ND } \\
--\end{array}$ & $\begin{array}{l}-- \\
-- \\
40 \\
10 \\
--\end{array}$ & $\begin{array}{l}-- \\
-- \\
20 \\
\text { ND } \\
--\end{array}$ & $\begin{array}{l}-- \\
-- \\
30 \\
60 \\
--\end{array}$ \\
\hline $\begin{array}{l}74-10-11 \\
75-05-01 \\
75-08-15 \\
74-10-15 \\
74-11-27\end{array}$ & $\begin{array}{l}-- \\
-- \\
\text { NO } \\
\text { NO }\end{array}$ & $\begin{array}{l}-- \\
-- \\
-- \\
20\end{array}$ & $\begin{array}{l}-- \\
10 \\
-- \\
10\end{array}$ & $\begin{array}{l}-- \\
-- \\
40 \\
\text { ND }\end{array}$ \\
\hline $\begin{array}{l}75-05-05 \\
75-08-15 \\
74-10-15 \\
74-11-27 \\
75-02-13\end{array}$ & $\begin{array}{c}-- \\
\text { NO } \\
-- \\
1\end{array}$ & $\begin{array}{l}-- \\
\text { ND } \\
-- \\
10\end{array}$ & $\begin{array}{l}-- \\
20 \\
-- \\
\text { ND }\end{array}$ & $\begin{array}{l}=- \\
30 \\
-- \\
30 \\
10\end{array}$ \\
\hline $\begin{array}{l}75-05-05 \\
75-08-18 \\
75-12-09 \\
75-12-09 \\
74-11-26\end{array}$ & $\begin{array}{l}-- \\
\text { ND } \\
-- \\
\text { ND }\end{array}$ & $\begin{array}{l}-- \\
10 \\
\text { ND } \\
10\end{array}$ & $\begin{array}{l}-- \\
10 \\
10 \\
--\end{array}$ & $\begin{array}{l}-- \\
20 \\
20 \\
-- \\
\text { NO }\end{array}$ \\
\hline
\end{tabular}


water from wezzs--Continued

\begin{tabular}{|c|c|c|c|c|}
\hline $\begin{array}{c}\text { OIS- } \\
\text { SOLVED } \\
\text { MERCURY } \\
\text { (HG) } \\
\text { (UG/L) }\end{array}$ & $\begin{array}{l}\text { DIS- } \\
\text { SOLVEO } \\
\text { NICKEL } \\
\text { (NI) } \\
(U G / L)\end{array}$ & $\begin{array}{l}\text { DIS- } \\
\text { SOLVED } \\
\text { ZINC } \\
\text { (ZN) } \\
(U G / L)\end{array}$ & $\begin{array}{l}\text { CODE } \\
\text { FOR } \\
\text { AGENCY } \\
\text { ANA- } \\
\text { LYZING } \\
\text { SAMPLE }\end{array}$ & $\begin{array}{c}\text { TOTAL } \\
\text { DEPTH } \\
\text { OF } \\
\text { WELL } \\
\text { (FT) }\end{array}$ \\
\hline $\begin{array}{l}\ldots \\
\therefore\end{array}$ & $\ddot{\text { ND }}$ & $\begin{array}{l}-\infty \\
-\infty\end{array}$ & $\begin{array}{l}9999 \\
9999 \\
9999\end{array}$ & $635^{--}$ \\
\hline $\begin{array}{l}= \\
=- \\
=- \\
=\end{array}$ & $\begin{array}{l}\text { ND } \\
-- \\
10 \\
10 \\
10\end{array}$ & $\begin{array}{l}\text { ND } \\
-0 \\
\text { ND } \\
100 \\
100\end{array}$ & $\begin{array}{l}9999 \\
9999 \\
9999 \\
9999 \\
9999\end{array}$ & $\begin{array}{r}635 \\
635 \\
635 \\
21 \\
21\end{array}$ \\
\hline $\begin{array}{l}-\infty \\
-\infty \\
-\infty \\
-\infty\end{array}$ & $\begin{array}{l}20 \\
N D \\
40 \\
20\end{array}$ & $\begin{array}{r}-- \\
60 \\
50 \\
100 \\
\text { ND }\end{array}$ & $\begin{array}{l}9999 \\
9999 \\
9999 \\
9999 \\
9999\end{array}$ & $\begin{array}{l}19 \\
19 \\
19 \\
19 \\
19\end{array}$ \\
\hline $\begin{array}{l}-\infty \\
-\infty \\
-\infty \\
-\infty\end{array}$ & $\begin{array}{l}-- \\
\text { ND } \\
-\infty \\
20 \\
10\end{array}$ & $\begin{array}{l}-2 \\
80 \\
10 \\
10 \\
10\end{array}$ & $\begin{array}{r}9999 \\
9999 \\
9999\end{array}$ & $\begin{array}{l}19 \\
19 \\
19 \\
20 \\
20\end{array}$ \\
\hline $\begin{array}{l}-\infty \\
-\infty \\
-\infty \\
-\infty\end{array}$ & $\begin{array}{l}-\infty \\
50 \\
\text { NO } \\
-\infty\end{array}$ & $\begin{array}{l}-\infty \\
-\infty \\
20 \\
\text { ND } \\
-\infty\end{array}$ & $\begin{array}{r}9999 \\
9999 \\
9999 \\
-\end{array}$ & $\begin{array}{l}20 \\
20 \\
20 \\
20 \\
20\end{array}$ \\
\hline $\begin{array}{l}=- \\
=- \\
=- \\
=-\end{array}$ & $\begin{array}{l}-- \\
20 \\
\cdots \\
\text { NO }\end{array}$ & $\begin{array}{r}=- \\
300 \\
=-\infty \\
110\end{array}$ & $\begin{array}{l}9999 \\
9999 \\
9999 \\
9999 \\
9999\end{array}$ & $\begin{array}{r}33 \\
33 \\
33 \\
573 \\
573\end{array}$ \\
\hline $\begin{array}{l}= \\
=- \\
-\infty \\
-\infty\end{array}$ & $\begin{array}{l}-- \\
\text { ND } \\
-- \\
\text { ND } \\
\text { ND }\end{array}$ & $\begin{array}{l}-\infty \\
60 \\
-0 \\
500 \\
480\end{array}$ & $\begin{array}{l}9999 \\
9999 \\
9999 \\
9999 \\
9999\end{array}$ & $\begin{array}{l}573 \\
573 \\
300 \\
300 \\
300\end{array}$ \\
\hline $\begin{array}{l}-- \\
\therefore \\
\therefore \\
\therefore\end{array}$ & $\begin{array}{l}-- \\
40 \\
\text { ND } \\
-- \\
\text { ND }\end{array}$ & $\begin{array}{r}100 \\
40 \\
-- \\
30\end{array}$ & $\begin{array}{r}9999 \\
9999 \\
9999 \\
9999 \\
999\end{array}$ & $\begin{array}{r}300 \\
300 \\
300 \\
300 \\
23\end{array}$ \\
\hline
\end{tabular}


Table 5.--Chemical analyses of

LOCAL
IDENT-
I-
FIER

SC00406530BDD

SC004065300CD

SC004065300DD

SC00406531AAA

SC00406531ACC

SC00406531DBB

SC0040653100C

SC00406532ADA

$$
\begin{gathered}
\text { OATE } \\
\text { OF } \\
\text { SAMPLE }
\end{gathered}
$$

TOTAL
DEPTH
OF

WELL

(FT)

DIS-
SOLVED
SILICA
(SIOZ)
(MG/L)

OIS-

SOLVED IRON (FE) (UG/L)

$$
\begin{array}{ll}
75-02-13 & 23 \\
75-05-07 & 23 \\
75-08-18 & 23 \\
75-12-09 & 23 \\
75-12-09 & 23 \\
76-04-05 & 23 \\
76-04-16 & 23 \\
74-11-26 & 18 \\
75-02-13 & 18
\end{array}
$$$$
\begin{array}{ll}
75-02-13 & 18 \\
75-05-07 & 18
\end{array}
$$$$
\text { 75-08-18 } \quad 18
$$$$
75-12-09 \quad 18
$$$$
75-12-09 \quad 18
$$$$
\text { 76-04-05 } 18
$$$$
74-11-26 \quad 16
$$$$
\text { 75-08-18 } \quad 16
$$$$
75-12-07 \quad 16
$$$$
\text { 75-12-07 } 11
$$$$
75-06-19 \quad 102
$$$$
75-08-19 \quad 102
$$$$
\text { 75-12-08 } 102
$$$$
\text { 75-12-08 } \quad 102
$$$$
\text { 76-04-02 } 102
$$$$
\text { 74-11-26 } 23
$$$$
\text { 75-02-12 } 23
$$$$
\text { 75-08-18 } 23
$$$$
\text { 75-12-07 } 23
$$$$
\text { 75-12-08 }
$$$$
76-03-30
$$$$
74-11-26
$$$$
23
$$$$
23
$$$$
\text { 75-02-12 }
$$$$
\text { 75-05-01 }
$$$$
75-08-18
$$$$
\text { 75-12-09 }
$$$$
\text { 75-12-09 }
$$$$
28
$$$$
28
$$$$
28
$$$$
28
$$$$
28
$$$$
28
$$$$
\text { 76-03-31 }
$$$$
\text { 76-04-16 }
$$$$
74-11-26
$$$$
75-02-12
$$$$
\text { 75-05-02 }
$$

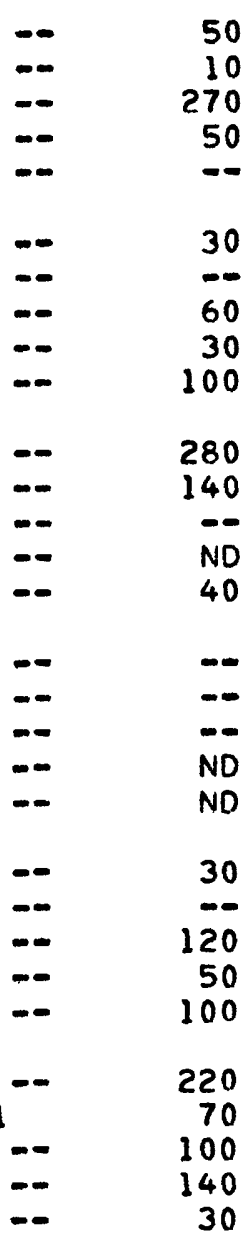

$=\quad 30$

$\begin{array}{rr}- & 1870 \\ - & 1480 \\ - & 2500 \\ -- & 270 \\ & -- \\ - & 300 \\ - & -0 \\ - & 600 \\ - & 2300\end{array}$


water from wells--Continued

$\begin{array}{cccccc} & & \text { OIS- } & & \text { DIS- } \\ \text { DIS- } & \text { OIS- } & \text { SOLVED } & & \text { SOLVED } \\ \text { SOLVED } & \text { SOLVED } & \text { MAG- } & \text { OIS- } & \text { PO- } & \\ \text { MAN- } & \text { CAL- } & \text { NE- } & \text { SOLVED } & \text { TAS- } & \text { BICAR- } \\ \text { GANESE } & \text { CIUM } & \text { SIUM } & \text { SODIUM } & \text { SIUM } & \text { BONATE } \\ \text { (MN) } & \text { (CA) } & \text { (MG) } & \text { (NA) } & \text { (K) } & \text { (HCOS) } \\ (U G / L) & (M G / L) & (M G / L) & \text { (MG/L) } & (M G / L) & \text { (MG/L) }\end{array}$

\begin{tabular}{|c|c|c|c|c|c|}
\hline $\begin{array}{r}880 \\
-- \\
670 \\
830\end{array}$ & $\begin{array}{r}91 \\
94 \\
103 \\
88\end{array}$ & $\begin{array}{l}=- \\
-\infty \\
=\end{array}$ & $\begin{array}{l}39 \\
47 \\
38 \\
35\end{array}$ & $\begin{array}{r}3.5 \\
-0 \\
9.0 \\
5.2\end{array}$ & $\begin{array}{r}259 \\
-2 \\
256 \\
368\end{array}$ \\
\hline-- & $-\infty$ & 9.5 & - & - & $-\infty$ \\
\hline 70 & & 9.5 & 37 & $\begin{array}{r}4.0 \\
--\end{array}$ & 246 \\
\hline $\begin{array}{l}1350 \\
2590\end{array}$ & $\begin{array}{l}58 \\
90\end{array}$ & $=$ & $\begin{array}{l}73 \\
27 \\
29\end{array}$ & $\begin{array}{l}4.9 \\
2.9\end{array}$ & $\begin{array}{l}287 \\
176\end{array}$ \\
\hline- & 10 & -- & & 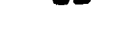 & $-\infty$ \\
\hline $\begin{array}{l}1650 \\
1700\end{array}$ & $\begin{array}{l}45 \\
82\end{array}$ & $=$ & $\begin{array}{l}26 \\
28\end{array}$ & $\begin{array}{l}7.0 \\
4.9\end{array}$ & $\begin{array}{l}192 \\
216\end{array}$ \\
\hline $\begin{array}{l}110 \\
640\end{array}$ & $\begin{array}{l}82^{--} \\
73^{-}\end{array}$ & $\begin{array}{r}9.0 \\
=-\end{array}$ & $\begin{array}{l}28 \\
55\end{array}$ & $\begin{array}{l}-. \\
4.0 \\
7.5\end{array}$ & $\begin{array}{l}192 \\
280\end{array}$ \\
\hline$=$ & $-\infty$ & -- & $-\infty$ & $\therefore$ & $\begin{array}{l}345 \\
476\end{array}$ \\
\hline$\because$ & $-\infty$ & 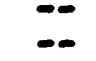 & $-\infty$ & $\ldots$ & 168 \\
\hline 10 & $\begin{array}{r}78 \\
134\end{array}$ & $-\infty$ & 67 & $\begin{array}{l}15 \\
13\end{array}$ & ND \\
\hline nn & & - & & 6.4 & 36 \\
\hline $\begin{array}{l}\text { ND } \\
--\end{array}$ & $\begin{array}{r}3.7 \\
\therefore-\end{array}$ & .1 & $51 \ldots$ & $\begin{array}{r}6.4 \\
-.-\end{array}$ & 50 \\
\hline 10 & 67 & .2 & 57 & 8.0 & ND \\
\hline $\begin{array}{l}540 \\
410\end{array}$ & $\begin{array}{l}60 \\
83\end{array}$ & $-\infty$ & $\begin{array}{l}85 \\
60\end{array}$ & $\begin{array}{l}6.6 \\
3.6\end{array}$ & $\begin{array}{l}170 \\
157\end{array}$ \\
\hline 830 & 97 & - & 36 & 6.0 & 173 \\
\hline $\begin{array}{l}750 \\
640\end{array}$ & 78 & 7.1 & $\begin{array}{l}48 \\
43\end{array}$ & $\begin{array}{l}3.1 \\
4.3\end{array}$ & $\begin{array}{l}212 \\
170\end{array}$ \\
\hline 410 & $\begin{array}{l}66 \\
70\end{array}$ & 7.1 & 48 & 5.0 & 166 \\
\hline 800 & 52 & $\infty$ & 55 & 21 & 263 \\
\hline 2880 & 96 & $-\infty$ & 35 & 3.6 & 244 \\
\hline & 75 & $-\infty$ & 61 & $-\infty$ & -- \\
\hline $\begin{array}{l}2200 \\
1080\end{array}$ & $\begin{array}{l}81 \\
76\end{array}$ & & $\begin{array}{l}35 \\
28\end{array}$ & $\begin{array}{l}12 \\
5.0\end{array}$ & $\begin{array}{l}217 \\
198\end{array}$ \\
\hline- & $=$ & 8.3 & - & - & $-\infty$ \\
\hline 910 & 74 & 8.0 & 32 & 6.0 & 192 \\
\hline-- & - & $-\infty$ & & & 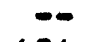 \\
\hline $\begin{array}{r}300 \\
3000\end{array}$ & $\begin{array}{l}400 \\
461\end{array}$ & $-\infty$ & $\begin{array}{l}190 \\
320\end{array}$ & $\begin{array}{l}25 \\
9.5\end{array}$ & $\begin{array}{l}434 \\
495\end{array}$ \\
\hline$-\infty$ & 532 & -- & 330 & - & $-\infty$ \\
\hline
\end{tabular}


Table 5.--Chemical analyses of

\begin{tabular}{|c|c|c|c|c|c|}
\hline $\begin{array}{c}\text { DATE } \\
\text { OF } \\
\text { SAMPLE }\end{array}$ & $\begin{array}{l}\text { CAR- } \\
\text { BONATE } \\
\text { (CO3) } \\
\text { (MG/L) }\end{array}$ & $\begin{array}{l}\text { HY- } \\
\text { DROX- } \\
\text { IDE } \\
(O H) \\
(M G / L)\end{array}$ & $\begin{array}{l}\text { ALKA- } \\
\text { LINITY } \\
\text { AS } \\
\text { CACO3 } \\
\text { (MG/L) }\end{array}$ & $\begin{array}{l}\text { DIS- } \\
\text { SOLVED } \\
\text { SULFATE } \\
(S 04) \\
(M G / L)\end{array}$ & $\begin{array}{l}\text { OIS- } \\
\text { SOLVED } \\
\text { CHLO- } \\
\text { RIDE } \\
\text { (CL) } \\
\text { (MG/L) }\end{array}$ \\
\hline $\begin{array}{l}75-02-13 \\
75-05-07 \\
75-08-18 \\
75-12-09 \\
75-12-09\end{array}$ & $\begin{array}{l}-- \\
\text { ND } \\
\text { ND } \\
--\end{array}$ & $\begin{array}{l}-- \\
-- \\
\text { ND } \\
\text { ND } \\
--\end{array}$ & $\begin{array}{r}212 \\
210 \\
302 \\
--\end{array}$ & $\begin{array}{l}77^{--} \\
84^{--}\end{array}$ & $\begin{array}{l}6.0 \\
9.0 \\
9.0 \\
7.0 \\
\ldots\end{array}$ \\
\hline $\begin{array}{l}76-04-05 \\
76-04-16 \\
74-11-26 \\
75-02-13 \\
75-05-07\end{array}$ & $\begin{array}{l}\text { ND } \\
\text { ND } \\
-- \\
--\end{array}$ & $\begin{array}{l}\text { NO } \\
=- \\
--\end{array}$ & $\begin{array}{r}202 \\
-0 \\
235 \\
144 \\
--\end{array}$ & $\begin{array}{l}65 \\
- \\
-\infty\end{array}$ & $\begin{array}{l}8.0 \\
34 \\
29 \\
32\end{array}$ \\
\hline $\begin{array}{l}75-08-18 \\
75-12-09 \\
75-12-09 \\
76-04-05 \\
74-11-26\end{array}$ & $\begin{array}{l}\text { ND } \\
\text { ND } \\
-- \\
\text { ND } \\
\text { ND }\end{array}$ & $\begin{array}{l}\text { ND } \\
\text { ND } \\
-- \\
\text { ND } \\
--\end{array}$ & $\begin{array}{l}157 \\
177 \\
-- \\
157 \\
230\end{array}$ & $\begin{array}{l}74 \\
94 \\
74^{-} \\
\ldots\end{array}$ & $\begin{array}{l}30 \\
24 \\
22^{-}\end{array}$ \\
\hline $\begin{array}{l}75-08-18 \\
75-12-07 \\
75-12-07 \\
75-06-19 \\
75-08-19\end{array}$ & $\begin{array}{r}\text { ND } \\
\text { ND } \\
\text { ND } \\
8 \\
40\end{array}$ & $\begin{array}{l}\text { ND } \\
\text { ND } \\
\text { ND } \\
444 \\
221\end{array}$ & $\begin{array}{r}283 \\
349 \\
138 \\
\ldots- \\
\ldots-\end{array}$ & $2150^{-\infty}$ & $\begin{array}{l}2.0 \\
21 \\
20\end{array}$ \\
\hline $\begin{array}{l}75-12-08 \\
75-12-08 \\
76-04-02 \\
74-11-26 \\
75-02-12\end{array}$ & $\begin{array}{l}\text { ND } \\
-- \\
36 \\
\text { NO } \\
--\end{array}$ & $\begin{array}{r}8 \\
-- \\
88 \\
--\end{array}$ & $\begin{array}{l}30 \\
=- \\
-- \\
139 \\
129\end{array}$ & $210^{-\infty}$ & $\begin{array}{l}16 \\
20^{-} \\
12 \\
21\end{array}$ \\
\hline $\begin{array}{l}75-08-18 \\
75-12-07 \\
75-12-08 \\
76-03-30 \\
74-11-26\end{array}$ & $\begin{array}{l}\text { ND } \\
\text { ND } \\
\text { ND } \\
\text { ND } \\
\text { ND }\end{array}$ & $\begin{array}{l}\text { ND } \\
-- \\
\text { NO } \\
\text { NO } \\
--\end{array}$ & $\begin{array}{l}142 \\
174 \\
139 \\
136 \\
216\end{array}$ & $\begin{array}{l}230 \\
140 \\
200 \\
129\end{array}$ & $\begin{array}{l}18 \\
15 \\
16 \\
21 \\
5.2\end{array}$ \\
\hline $\begin{array}{l}75-02-12 \\
75-05-01 \\
75-08-18 \\
75-12-09 \\
75-12-09\end{array}$ & $\begin{array}{l}-- \\
-- \\
\text { ND } \\
\text { ND } \\
--\end{array}$ & $\begin{array}{l}-- \\
-- \\
\text { ND } \\
--\end{array}$ & $\begin{array}{r}200 \\
-- \\
178 \\
162 \\
--\end{array}$ & $\begin{array}{c}86^{--} \\
104^{--}\end{array}$ & $\begin{array}{r}10 \\
5.0 \\
7.0 \\
4.0 \\
=-\end{array}$ \\
\hline $\begin{array}{l}76-03-31 \\
76-04-16 \\
74-11-26 \\
75-02-12 \\
75-05-02\end{array}$ & $\begin{array}{l}\text { ND } \\
-- \\
\text { ND } \\
--\end{array}$ & $\begin{array}{l}\text { ND } \\
-- \\
-- \\
-\infty\end{array}$ & $\begin{array}{r}157 \\
-- \\
356 \\
406 \\
--\end{array}$ & $\begin{array}{r}101 \\
\ldots \\
=- \\
=\end{array}$ & $\begin{array}{l}2.0 \\
25 \\
35 \\
29\end{array}$ \\
\hline
\end{tabular}


DIS-

SOLVED

FLUO-

RIDE

(F) \begin{tabular}{cccccc} 
DIS- & \multicolumn{1}{c}{ DIS } & DIS- & & DIS \\
SOLVED & TOTAL & SOLVED & SOLVED & TOTALI & SOLVED \\
NITRATE & NITRATE & NITRATE & NITRITE & NITRITE & NITRITE \\
$(N)$ & $(N O 3)$ & $(N O 3)$ & $(N)$ & $(N O 2)$ & $(N O 2)$ \\
$(M G / L)$ & $(M G / L)$ & $(M G / L)$ & $(M G / L)$ & $(M G / L)$ & $(M G / L)$
\end{tabular}

(MG/L)
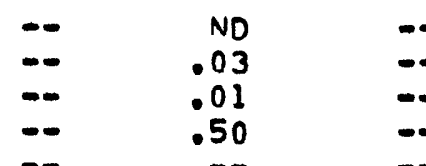

$-$

$-\infty$

$\begin{array}{ll}-.00 \\ \therefore & .11\end{array}$

$-\infty$

$-0$

.50

$-$

$=03$

-00
$-0 \quad 00$

-0.60
$-0 \quad .97$

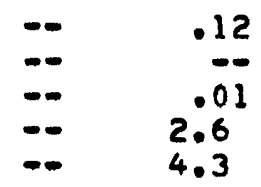

$-\infty$

$-$

.00

.00

.00

\section{$-0.70$}

$-.50$

$--\quad 3.1$
$--\quad 2.2$

.01

.06

$\begin{array}{lll}-\infty & -1 \\ -\infty & -\infty\end{array}$

$\ldots .51$

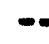

2.2

-

$-$

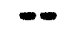

$\ddot{z}$

$=1.4$

$=0.08$

$-. \quad .05$

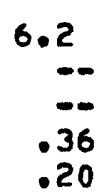

.14

$-$

.09

.00

$-\infty \quad$ NN

ND

Nก

NO

0.00

.01

.01

$--$

.00

.00

$\because$

$-$

$\therefore \quad .02$

$=00$

.08

ND

$--$

$-\infty$

Nก

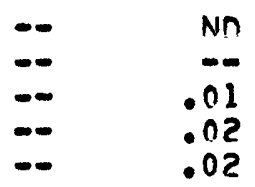

$\ldots .00$

$-$

$-$

- $\quad .02$

.01

$-\infty$

$-$

$-0.06$

$-20$

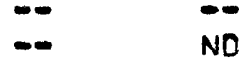

-. $\quad .01$

$\ldots \quad .03$

$\therefore \quad .05$

$-0.03$

$-$

$\ldots$

$\ldots .01$

$\because$

-.03
-.02

.15

$-$

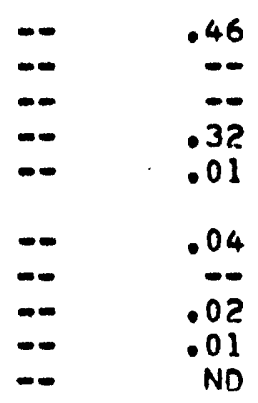

-. $\quad .01$

- $\quad .02$

-- $\quad .07$

-0.05

- .11

.00

$-$

$-\infty$

$-\infty \quad$ ND

$\because .03$

$-\infty \quad$ ND

- ND

ND

$-\infty \quad \ldots$

$-\infty$

$-$

$-\quad$ NN

Nn
.01

Nก

ND

$\begin{array}{lr}-0 & .01 \\ -- & 00 \\ -\infty & .00\end{array}$

$\because=$

.06
.02
.02
.05

$\because$
$\because-$

$\because \quad$ ND

ND

ND

ND 
Table 5.--Chemical analyses of

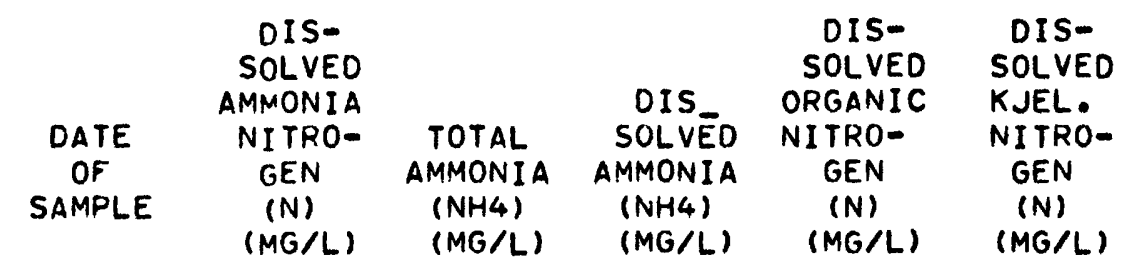

$75-02-13$
$75-05-07$
$75-08-18$
$75-12-09$
$75-12-09$
$76-04-05$
$76-04-16$
$74-11-26$
$75-02-13$
$75-05-07$

NO

ND

ND

ND

-

ND

-

ND

ND

.20

$75-08-18$

75-12-09

75-12-09

76-04-05

$74-11-26$

NO

ND

$-$

ND

ND

75-08-18

$75-12-07$

75-12-07

75-06-19

75-08-19

.90

$-\infty$

.10

.40

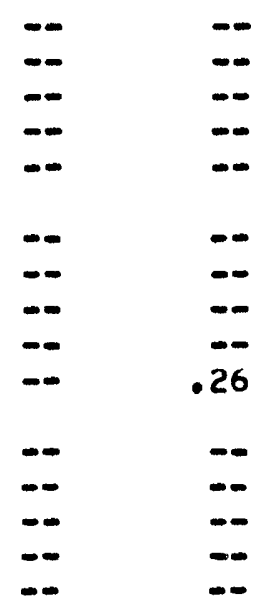

$-\infty$
$-\infty$
$-\infty$
$-\infty$

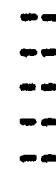

1.0

9.5

.20

D

$-$

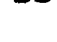

75-12-08

$75-12-08$

76-04-02

$74-11-26$

75-02-12

ND

$-$

.10

ND

ND

$-$

$-$

.00

.20
.0
5.6
1.1
.20

75-08-18

$75-12-07$

$75-12-08$

$76-03-30$

$74-11-26$

ND

.07

ND

ND

NO

75-02-12

.30

75-05-01

.30

.50

$75-08-18$
$75-12-09$

75-12-09

ND

$-$

.40

$-$

$-$

$-\infty$

.20

.60

5.8

$76-03-31$

$76-04-16$

$74-11-26$

$75-02-12$

75-05-02

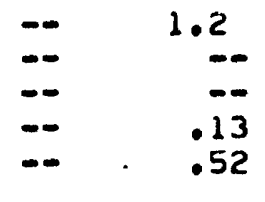

$-$

$-$

.20

$-\infty$

.80

.30

1.2

$-$

.20

$\begin{array}{lll}-\infty & -\infty\end{array}$

$\begin{array}{ll}-0 & 13 \\ -- & --\end{array}$

$\begin{array}{ll}-0 & .13 \\ -- & --\end{array}$

.50

$-\infty$

--

.60

ND

$\Rightarrow \quad 1.8$

$\begin{array}{ll}-\infty & -0 \\ -\infty & 09 \\ -\infty & -\infty\end{array}$

.22

.30

.29

$-$

.20

$\ldots$

ND

9.7

-- $\quad .39$

.70

.40

1.0

.39

.40

.70

.- .64

$=$

$-\infty$

.90

ND

NO

$-\infty$

2.4

.40
2.3
2.3
.60 
water from wells--Continued

$\begin{array}{clccccc}\text { DIS- } & \text { DIS- } & \text { DIS- } & \text { DIS- } & \text { DIS- } & & \\ \text { SOLVED } & \text { SOLVED } & \text { SOLVED } & \text { SOLVED } & \text { SOLVED } & & \text { NON- } \\ \text { ORTHO. } & \text { ORTHO } & \text { SOLIDS } & \text { SOLIDS } & \text { SOLIDS } & & \text { CAR- } \\ \text { PHOS- } & \text { PHOS- } & \text { (RESI- } & \text { (RESI- } & \text { (SUM OF } & \text { HARD- } & \text { BONATE } \\ \text { PHORUS } & \text { PHATE } & \text { DUE AT } & \text { DUE AT } & \text { CONSTI- } & \text { NESS } & \text { HARD- } \\ (P) & (P 04) & \left.180^{\circ} C\right) & \left.105^{\circ} C\right) & \text { TUENTS) } & \text { (CA,MG) } & \text { NESS } \\ (M G / L) & (M G / L) & (M G / L) & (M G / L) & \text { (MG/L) } & \text { (MG/L) } & \text { (MG/L) }\end{array}$

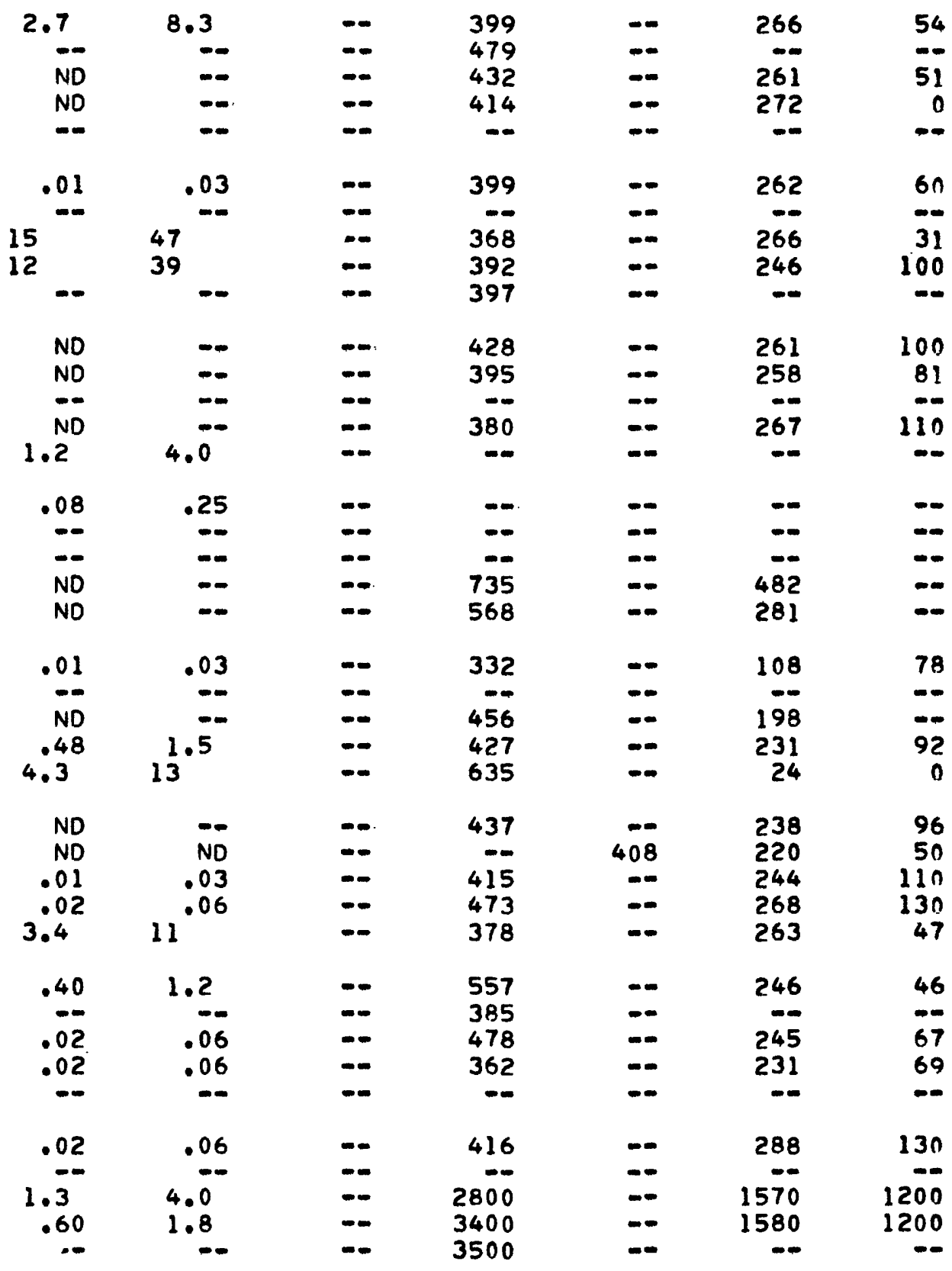


Table 5.--Chemical analyses of

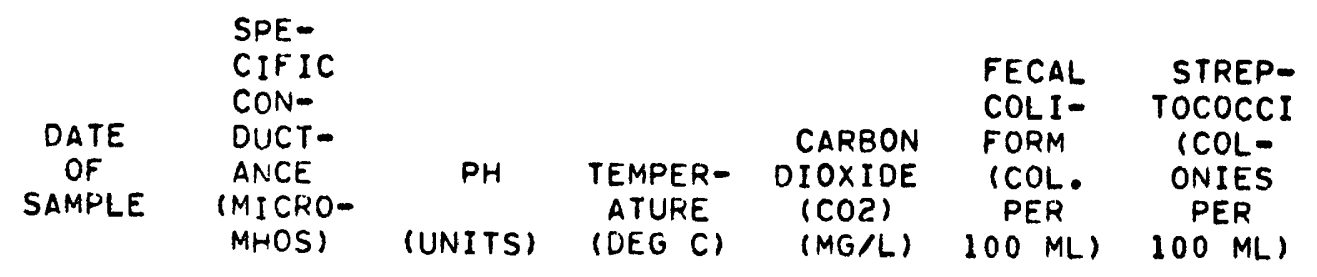

\begin{tabular}{|c|c|c|c|c|c|c|}
\hline $\begin{array}{l}75-02-13 \\
75-05-07 \\
75-08-18 \\
75-12-09 \\
75-12-09\end{array}$ & $\begin{array}{l}675 \\
700 \\
710 \\
710 \\
710\end{array}$ & $\begin{array}{l}7.2 \\
7.5 \\
7.3 \\
7.6 \\
7.6\end{array}$ & $\begin{array}{r}12.0 \\
8.5 \\
15.5 \\
12.5 \\
12.5\end{array}$ & $\begin{array}{l}26 \\
21 \\
15 \\
-\end{array}$ & $\begin{array}{l}0 \\
-- \\
-- \\
-\infty\end{array}$ & $\begin{array}{r}177 \\
-- \\
--\end{array}$ \\
\hline $\begin{array}{l}76-04-05 \\
76-04-16 \\
74-11-26 \\
75-02-13 \\
75-05-07\end{array}$ & $\begin{array}{l}650 \\
-5 \\
660 \\
650 \\
675\end{array}$ & $\begin{array}{r}7.5 \\
-0 \\
7.5 \\
7.5 \\
7.5\end{array}$ & $\begin{array}{r}13.0 \\
-0.0 \\
12.0 \\
10.5 \\
8.5\end{array}$ & $\begin{array}{c}12 \\
15 \\
8.9 \\
--\end{array}$ & $\begin{array}{l}-\infty \\
-\infty \\
-\infty\end{array}$ & $\begin{array}{r}-\infty \\
-\infty \\
-\infty\end{array}$ \\
\hline $\begin{array}{l}75-08-18 \\
75-12-09 \\
75-12-09 \\
76-04-05 \\
74-11-26\end{array}$ & $\begin{array}{l}710 \\
700 \\
700 \\
580 \\
800\end{array}$ & $\begin{array}{l}7.6 \\
7.8 \\
7.8 \\
7.8 \\
7.0\end{array}$ & $\begin{array}{r}14.5 \\
13.5 \\
13.5 \\
11.0 \\
9.5\end{array}$ & $\begin{array}{l}7.7 \\
5.5 \\
-.5 \\
45\end{array}$ & $\begin{array}{l}m \\
=- \\
=- \\
-\infty\end{array}$ & $\begin{array}{l}-\infty \\
=- \\
--\end{array}$ \\
\hline $\begin{array}{l}75-08-18 \\
75-12-07 \\
75-12-07 \\
75-06-19 \\
75-08-19\end{array}$ & $\begin{array}{r}925 \\
875 \\
1750 \\
2800 \\
1600\end{array}$ & $\begin{array}{r}7.5 \\
7.4 \\
7.5 \\
11.7 \\
11.5\end{array}$ & $\begin{array}{l}15.5 \\
12.0 \\
10.5 \\
14.5 \\
14.0\end{array}$ & $\begin{array}{r}17 \\
27 \\
8.5 \\
-.-\end{array}$ & $\begin{array}{l}= \\
-\infty \\
-\infty \\
-\infty\end{array}$ & $\begin{array}{l}-\infty \\
-\infty \\
-\infty \\
-\infty\end{array}$ \\
\hline $\begin{array}{l}75-12-08 \\
75-12-08 \\
76-04-02 \\
74-11-26 \\
75-02-12\end{array}$ & $\begin{array}{l}560 \\
560 \\
800 \\
650 \\
700\end{array}$ & $\begin{array}{l}10.1 \\
10.1 \\
11.1 \\
7.3 \\
7.5\end{array}$ & $\begin{array}{l}11.0 \\
11.0 \\
13.5 \\
10.5 \\
10.5\end{array}$ & $\begin{array}{r}.0 \\
14^{-0} \\
7.9\end{array}$ & $\begin{array}{l}= \\
=- \\
= \\
0\end{array}$ & $\begin{array}{l}-\infty \\
=- \\
-\infty \\
0\end{array}$ \\
\hline $\begin{array}{l}75-08-18 \\
75-12-07 \\
75-12-08 \\
76-03-30 \\
74-11-26\end{array}$ & $\begin{array}{r}740 \\
1750 \\
740 \\
625 \\
675\end{array}$ & $\begin{array}{l}7.7 \\
7.5 \\
7.0 \\
7.9 \\
7.0\end{array}$ & $\begin{array}{r}12.5 \\
10.5 \\
10.5 \\
9.0 \\
11.0\end{array}$ & $\begin{array}{l}5.5 \\
11 \\
27 \\
3.3 \\
42\end{array}$ & $\begin{array}{l}-\infty \\
-\infty \\
-\infty \\
--\end{array}$ & $\begin{array}{l}-- \\
-- \\
--\end{array}$ \\
\hline $\begin{array}{l}75-02-12 \\
75-05-01 \\
75-08-18 \\
75-12-09 \\
75-12-09\end{array}$ & $\begin{array}{l}660 \\
650 \\
675 \\
640 \\
640\end{array}$ & $\begin{array}{l}7.0 \\
7.6 \\
7.7 \\
7.9 \\
7.9\end{array}$ & $\begin{array}{l}11.5 \\
10.0 \\
13.5 \\
12.0 \\
12.0\end{array}$ & $\begin{array}{r}39 \\
-.0 \\
6.9 \\
4.0 \\
--\end{array}$ & $\begin{array}{l}0 \\
-\infty \\
-\infty \\
-\infty\end{array}$ & $\begin{array}{l}49 \\
-- \\
-- \\
-\infty\end{array}$ \\
\hline $\begin{array}{l}76-03-31 \\
76-04-16 \\
74-11-26 \\
75-02-12 \\
75-05-02\end{array}$ & $\begin{array}{r}560 \\
-- \\
3400 \\
4000 \\
5500\end{array}$ & $\begin{array}{r}7.6 \\
--0 \\
7.0 \\
6.6 \\
7.0\end{array}$ & $\begin{array}{r}11.0 \\
-0 \\
11.0 \\
12.0 \\
13.5\end{array}$ & $\begin{array}{r}7.7 \\
69 \\
199 \\
-\end{array}$ & $\begin{array}{l}-\infty \\
-\infty \\
-\infty \\
-\infty\end{array}$ & $\begin{array}{r}-\infty \\
-\infty \\
-\infty\end{array}$ \\
\hline
\end{tabular}


water from wells--Continued

\begin{tabular}{|c|c|c|c|c|c|}
\hline $\begin{array}{c}\text { CYANIDE } \\
\text { (CN) } \\
\text { (MG/L) }\end{array}$ & $\begin{array}{l}\text { PHENOLS } \\
\text { (UG/L) }\end{array}$ & $\begin{array}{l}\text { TOTAL } \\
\text { ALDRIN } \\
\text { (UG/L) }\end{array}$ & $\begin{array}{l}\text { TOTAL } \\
\text { CHLOR- } \\
\text { DANE } \\
\text { (UG/L) }\end{array}$ & $\begin{array}{l}\text { TOTAL } \\
\text { DDD } \\
\text { (UG/L) }\end{array}$ & $\begin{array}{l}\text { TOTAL } \\
\text { DDE } \\
\text { (UG/L) }\end{array}$ \\
\hline-- & - & $-\infty$ & - & - & \\
\hline$=-$ & - & -- & $\ldots$ & - & - \\
\hline$-\infty$ & $-\infty$ & NO & ND & ND & ND \\
\hline- & - & $\ldots$ & $-\infty$ & $=-$ & $=$ \\
\hline- & $-\infty$ & $-\infty$ & $-\infty$ & $-\infty$ & - \\
\hline- & -- & -- & -- & - & - \\
\hline - & $=$ & $-\infty$ & $=$ & $=$ & $=-$ \\
\hline-- & -- & $-\infty$ & -- & - & 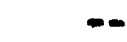 \\
\hline$=$ & $-\infty$ & $-\infty$ & -- & $-\infty$ & $-\infty$ \\
\hline-- & -- & $\infty$ & $-\infty$ & $-\infty$ & - \\
\hline$-\infty$ & $-\infty$ & $-\infty$ & $-\infty$ & - & $=$ \\
\hline$-\infty$ & -- & - & $-\infty$ & - & - \\
\hline- & -- & - & $=-$ & - & - \\
\hline$\cdots$ & $\cdots$ & -- & - & - & $-\infty$ \\
\hline$-\infty$ & $-\infty$ & -- & $-\infty$ & -- & $=$ \\
\hline- & $-\infty$ & $\ldots$ & $\ldots$ & - & 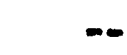 \\
\hline$\ldots$ & $-\infty$ & $\ldots$ & $\ldots$ & - & - \\
\hline$\cdots$ & $-\infty$ & -- & -- & - & - \\
\hline$-\infty$ & $-\infty$ & -- & -- & -- & - \\
\hline$-\infty$ & -- & -- & -- & -- & \\
\hline$-\infty$ & -- & $-\infty$ & 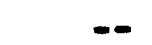 & $-\infty$ & - \\
\hline 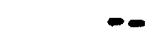 & $-\infty$ & -- & $\ldots$ & -- & - \\
\hline-- & -- & -- & -- & -- & - \\
\hline-- & $-\infty$ & - & $-\infty$ & - & - \\
\hline-- & $-\infty$ & -- & $-\infty$ & -- & \\
\hline$-\infty$ & $-\infty$ & No & ND & ND & ND \\
\hline 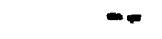 & $\cdots$ & 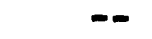 & 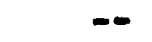 & - & - \\
\hline$m$ & $\cdots$ & $-\infty$ & $\cdots$ & $\cdots$ & \\
\hline- & -- & $-\infty$ & $-\infty$ & $-\infty$ & - \\
\hline- & -- & $-\infty$ & -- & $\cdots$ & \\
\hline -. & 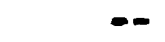 & -- & 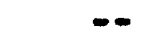 & -- & \\
\hline 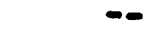 & $-\infty$ & $-\infty$ & $-\infty$ & - & \\
\hline$-\infty$ & $\infty$ & -- & $-\infty$ & - & \\
\hline$-\infty$ & $\infty$ & $-\infty$ & $\cdots$ & - & \\
\hline$-\infty$ & $\cdots$ & - & - & $\cdots$ & \\
\hline- & -- & - & - & - & \\
\hline$-\infty$ & - & - & $-\infty$ & -- & \\
\hline$\ldots$ & -- & -- & $-\infty$ & - & \\
\hline$=$ & - & $-\infty$ & $-\infty$ & $-\overline{-}$ & \\
\hline & & & & $=$ & \\
\hline
\end{tabular}


Table 5.--Chemical analyses of

\section{DATE \\ OF}

SAMPLE

$$
\begin{aligned}
& \text { TOTAL } \\
& \text { DOT }
\end{aligned}
$$

(UG/L)
TOTAL TOTAL

DI AZINON

(UG/L)
DIELDRIN (UG/L)
TOTAL

ENDRIN (UG/L)
TOTAL

HEPTA-

CHLOR

(UG/L)
TOTAL

HEPTA-

CHLOR

EPOXIDE

(UG/L)
75-02-13

75-05-07

75-08-18

$75-12-09$

75-12-09

76-04-05

76-04-16

$74-11-26$

75-02-13

75-05-07

$75-08-18$

75-12-09

75-12-09

76-04-05

74-11-26

$75-08-18$

75-12-07

75-12-07

75-06-19

75-08-19

$75-12-08$

$75-12-08$

$76-04-02$

$74-11-26$

75-02-12

$75-08-18$

75-12-07

$75-12-08$

76-03-30

$74-11-26$

$75-02-12$

75-05-01

$75-08-18$

75-12-09

75-12-09

$76-03-31$

76-04-16

$74-11-26$

$75-02-12$

75-05-02
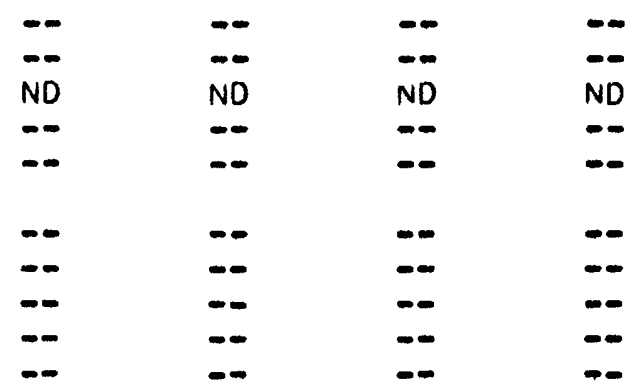

$\begin{array}{ll}-- & - \\ -- & = \\ -- & -\end{array}$

--

$-$

$-\infty$

$-\infty$

$-\infty \quad-$

-- $\quad-$

$-\infty$

$\overline{-\infty}$

$\ldots$

ND

--

$-$

$-$

$-$

$-\infty$

$=$

$-\infty$

$-$

$-\infty$

$-\infty$

$-\infty$

$-$

$-$

$-$

$-\infty$

$-\infty$

$-$

$-$

$-$

$-$

$-$

$-$

$-$

$-$

$-\infty$

$-$

$-$

$-$

$-$

$-$

$-\infty$

$-\infty$

$-$

$-$

$-$

$-$

$-\infty$

$-$

$-$

$-$

$-$

ND

ND

-

$-$

$-$

$-$

$-$

$-$

$-$

$-$

$-$

$-$

$-$

$-$

$-\infty$

$-\infty$

--
--
--

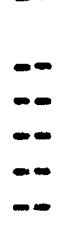

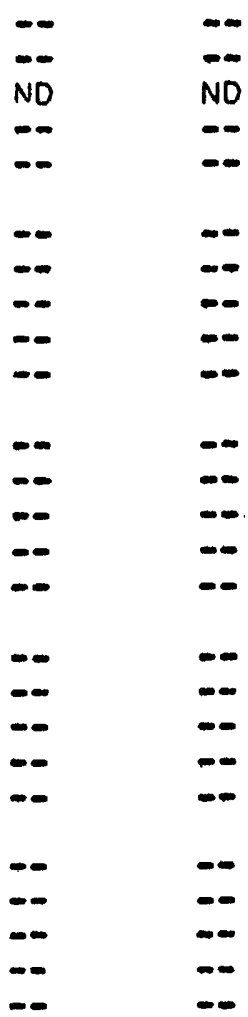

NO

$-$

$-$

$-$

$-$

--

$-\infty$

$-$

$-$

$-$

$=$

$-$ 
water from wells--Continued

\begin{tabular}{|c|c|c|c|c|c|}
\hline $\begin{array}{l}\text { TOTAL } \\
\text { LINDANE } \\
\text { (UG/L) }\end{array}$ & $\begin{array}{l}\text { TOTAL } \\
\text { MALA- } \\
\text { THION } \\
\text { (UG/L) }\end{array}$ & $\begin{array}{l}\text { TOTAL } \\
\text { METHYL } \\
\text { PARA- } \\
\text { THION } \\
\text { (UG/L) }\end{array}$ & $\begin{array}{l}\text { TOTAL } \\
\text { PARA- } \\
\text { THION } \\
\text { (UG/L) }\end{array}$ & $\begin{array}{l}\text { TOTAL } \\
\text { PCB } \\
\text { (UG/L) }\end{array}$ & $\begin{array}{l}\text { TOTAL } \\
\text { TOX- } \\
\text { APHENE } \\
\text { (UG/L) }\end{array}$ \\
\hline- & $-\infty$ & $-\infty$ & $-\infty$ & - & - \\
\hline- & $-\infty$ & $-\infty$ & $-\infty$ & - & - \\
\hline ND & ND & ND & NO & ND & ND \\
\hline- & -- & $-\infty$ & - & - & $=-$ \\
\hline- & $-\infty$ & $-\infty$ & $-\infty$ & - & $-\infty$ \\
\hline$-\infty$ & $-\infty$ & $\infty$ & - & - & - \\
\hline- & $-\infty$ & -- & -- & - & $=$ \\
\hline$-\infty$ & $=-$ & - & $=$ & - & 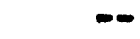 \\
\hline$m$ & $-\infty$ & - & - & - & 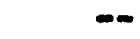 \\
\hline$-\infty$ & $-\infty$ & $-\infty$ & $-\infty$ & -- & $=$ \\
\hline- & - & $-\infty$ & - & - & $-\infty$ \\
\hline- & $-\infty$ & $-\infty$ & - & $-\infty$ & -- \\
\hline$-\infty$ & $=-$ & -- & $-\infty$ & $-\infty$ & $-\infty$ \\
\hline-- & $-\infty$ & $-\infty$ & $-\infty$ & $-\infty$ & - \\
\hline$-\infty$ & -- & $-\infty$ & - & - & $-\infty$ \\
\hline$-\infty$ & $-\infty$ & $\infty$ & $-\infty$ & - & $-\infty$ \\
\hline$m$ & $-\infty$ & $-\infty$ & $-\infty$ & 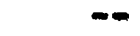 & - \\
\hline- & $-\infty$ & $-\infty$ & $-\infty$ & $=$ & - \\
\hline$\cdots$ & $-\infty$ & $-\infty$ & 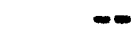 & - & - \\
\hline$-\infty$ & $-\infty$ & $\infty$ & $-\infty$ & - & $-\infty$ \\
\hline$-\infty$ & $-\infty$ & $-\infty$ & - & - & $\infty$ \\
\hline 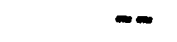 & -- & $-\infty$ & - & $-\infty$ & 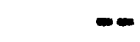 \\
\hline$-\infty$ & $-\infty$ & $-\infty$ & 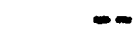 & $\cdots$ & - \\
\hline- & $-\infty$ & $-\infty$ & $-\infty$ & $-\infty$ & - \\
\hline-- & $-\infty$ & $\infty$ & - & $-\infty$ & - \\
\hline ND & ND & ND & ND & No & No \\
\hline$-\infty$ & $-\infty$ & $-\infty$ & $-\infty$ & $-\infty$ & - \\
\hline$-\infty$ & $-\infty$ & $-\infty$ & $\infty$ & $-\infty$ & - \\
\hline$=$ & - & $-\infty$ & 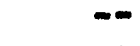 & $-\infty$ & $-\infty$ \\
\hline 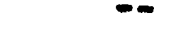 & $\infty$ & $-\infty$ & $-\infty$ & $-\infty$ & - \\
\hline$\infty$ & - & $-\infty$ & - & $-\infty$ & - \\
\hline$\infty$ & $-\infty$ & $-\infty$ & $m$ & - & $=$ \\
\hline$-\infty$ & - & $-\infty$ & $-\infty$ & $\because$ & - \\
\hline$=$ & - & $=-$ & 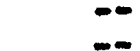 & 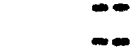 & 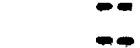 \\
\hline$-\infty$ & $-\infty$ & $-\infty$ & - & 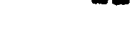 & \\
\hline$\sim$ & - & $-\infty$ & $-\infty$ & - & - \\
\hline$-\infty$ & -- & $-\infty$ & $-\infty$ & - & E \\
\hline- & - & $-\infty$ & $-\infty$ & $=-$ & - \\
\hline$-\infty$ & - & - & $-\infty$ & - & 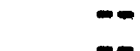 \\
\hline$-\infty$ & - & - & $\cdots$ & - & \\
\hline
\end{tabular}


Table 5.--Chemical analyses of

\begin{tabular}{|c|c|c|c|c|}
\hline $\begin{array}{c}\text { UATE } \\
\text { OF } \\
\text { SAMPLE }\end{array}$ & $\begin{array}{l}\text { DIS- } \\
\text { SOLVED } \\
\text { CAD- } \\
\text { MIUM } \\
\text { (CD) } \\
\text { (UG/L) }\end{array}$ & $\begin{array}{l}\text { DIS- } \\
\text { SOLVED } \\
\text { CHRO- } \\
\text { MIUM } \\
\text { (CR) } \\
\text { (UG/L) }\end{array}$ & $\begin{array}{l}\text { DIS- } \\
\text { SOLVED } \\
\text { COPPER } \\
\text { (CU) } \\
(U G / L)\end{array}$ & $\begin{array}{l}\text { DIS- } \\
\text { SOLVED } \\
\text { LEAD } \\
\text { (PA) } \\
\text { (UG/L) }\end{array}$ \\
\hline $\begin{array}{l}75-02-13 \\
75-05-07 \\
75-08-18 \\
75-12-09 \\
75-12-09\end{array}$ & $\begin{array}{r}\text { ND } \\
-- \\
5 \\
\text { ND } \\
--\end{array}$ & $\begin{array}{l}10 \\
-\infty \\
20 \\
10 \\
-\infty\end{array}$ & $\begin{array}{r}\text { ND } \\
-- \\
390 \\
\text { ND } \\
--\end{array}$ & $\begin{array}{l}\text { ND } \\
-- \\
30 \\
40 \\
--\end{array}$ \\
\hline $\begin{array}{l}76-04-05 \\
76-04-16 \\
74-11-26 \\
75-02-13 \\
75-05-07\end{array}$ & $\begin{array}{l}\text { ND } \\
-- \\
\text { ND } \\
\text { ND } \\
--\end{array}$ & $\begin{array}{l}\text { ND } \\
-- \\
\text { NO } \\
\text { ND } \\
--\end{array}$ & $\begin{array}{l}10 \\
-\infty \\
\text { ND } \\
\text { ND } \\
-\infty\end{array}$ & $\begin{array}{l}\text { ND } \\
\text { ND } \\
\text { ND } \\
--\end{array}$ \\
\hline $\begin{array}{l}75-08-18 \\
75-12-09 \\
75-12-09 \\
76-04-05 \\
74-11-26\end{array}$ & $\begin{array}{r}4 \\
6 \\
-- \\
\text { ND } \\
2\end{array}$ & $\begin{array}{l}20 \\
\text { ND } \\
-- \\
10 \\
\text { ND }\end{array}$ & $\begin{array}{r}170 \\
10 \\
-0 \\
10 \\
\text { NO }\end{array}$ & $\begin{array}{l}\text { ND } \\
40 \\
-\infty \\
\text { ND } \\
\text { ND }\end{array}$ \\
\hline $\begin{array}{l}75-08-18 \\
75-12-07 \\
75-12-07 \\
75-06-19 \\
75-08-19\end{array}$ & $\begin{array}{l}-- \\
-- \\
-- \\
\text { ND }\end{array}$ & $\begin{array}{l}-- \\
-- \\
\text { ND } \\
\text { ND }\end{array}$ & $\begin{array}{l}-\infty \\
-- \\
10 \\
50\end{array}$ & $\begin{array}{l}-- \\
-- \\
\text { ND } \\
50\end{array}$ \\
\hline $\begin{array}{l}75-12-08 \\
75-12-08 \\
76-04-02 \\
74-11-26 \\
75-02-12\end{array}$ & $\begin{array}{r}4 \\
-- \\
\text { ND } \\
2 \\
\text { ND }\end{array}$ & $\begin{array}{l}\text { ND } \\
-- \\
\text { ND } \\
10 \\
\text { ND }\end{array}$ & $\begin{array}{l}\text { ND } \\
-- \\
20 \\
\text { ND } \\
\text { ND }\end{array}$ & $\begin{array}{l}20 \\
-- \\
60 \\
\text { ND } \\
\text { ND }\end{array}$ \\
\hline $\begin{array}{l}75-08-18 \\
75-12-07 \\
75-12-08 \\
76-03-30 \\
74-11-26\end{array}$ & $\begin{array}{r}4 \\
-- \\
5 \\
\text { ND } \\
\text { ND }\end{array}$ & $\begin{array}{l}10 \\
=- \\
\text { ND } \\
10 \\
\text { ND }\end{array}$ & $\begin{array}{l}30 \\
-\infty \\
N D \\
10 \\
\text { ND }\end{array}$ & $\begin{array}{r}\text { ND } \\
-5 \\
40 \\
110 \\
30\end{array}$ \\
\hline $\begin{array}{l}75-02-12 \\
75-05-01 \\
75-08-18 \\
75-12-09 \\
75-12-09\end{array}$ & $\begin{array}{r}1 \\
-- \\
3 \\
--\end{array}$ & $\begin{array}{l}10 \\
-- \\
10 \\
\text { ND } \\
-\end{array}$ & $\begin{array}{l}10 \\
-- \\
10 \\
\text { ND } \\
--\end{array}$ & $\begin{array}{l}40 \\
-- \\
20 \\
\text { ND } \\
--\end{array}$ \\
\hline $\begin{array}{l}76-03-31 \\
76-04-16 \\
74-11-26 \\
75-02-12 \\
75-05-02\end{array}$ & $\begin{array}{c}\text { ND } \\
-- \\
1 \\
6 \\
--\end{array}$ & $\begin{array}{l}\text { NO } \\
-- \\
40 \\
10 \\
--\end{array}$ & $\begin{array}{l}\text { ND } \\
-- \\
\text { ND } \\
10 \\
--\end{array}$ & $\begin{array}{l}20 \\
-\infty \\
50 \\
50 \\
--\end{array}$ \\
\hline
\end{tabular}


water from wells--Continued

\begin{tabular}{|c|c|c|c|c|}
\hline $\begin{array}{l}\text { DIS- } \\
\text { SOLVEO } \\
\text { MERCURY } \\
\text { (HG) } \\
\text { (UG/L) }\end{array}$ & $\begin{array}{l}\text { DIS- } \\
\text { SOLVED } \\
\text { NICKEL } \\
\text { (NI) } \\
\text { (UG/L) }\end{array}$ & $\begin{array}{l}\text { DIS- } \\
\text { SOLVED } \\
\text { ZINC } \\
\text { (ZN) } \\
\text { (UG/L) }\end{array}$ & $\begin{array}{l}\text { CODE } \\
\text { FOR } \\
\text { AGENCY } \\
\text { ANA- } \\
\text { LYZING } \\
\text { SAMPLE }\end{array}$ & $\begin{array}{c}\text { TOTAL } \\
\text { DEPTI } \\
\text { OF } \\
\text { WELL } \\
\text { IFT }\end{array}$ \\
\hline $\begin{array}{l}-\infty \\
-\infty \\
=- \\
-\infty\end{array}$ & $\begin{array}{l}\text { ND } \\
-\infty \\
10 \\
10 \\
-\infty\end{array}$ & $\begin{array}{r}50 \\
-0 \\
160 \\
40 \\
-\end{array}$ & $\begin{array}{r}9999 \\
9999 \\
9999 \\
9999 \\
-\end{array}$ & $\begin{array}{l}23 \\
23 \\
23 \\
23 \\
23\end{array}$ \\
\hline $\begin{array}{l}= \\
= \\
= \\
=\end{array}$ & $\begin{array}{l}\text { ND } \\
-0 \\
20 \\
10 \\
-\infty\end{array}$ & $\begin{array}{l}30 \\
-0 \\
50 \\
10 \\
-\infty\end{array}$ & $\begin{array}{r}9999 \\
-0 \\
9999 \\
9999 \\
9999\end{array}$ & $\begin{array}{l}23 \\
23 \\
18 \\
18 \\
18\end{array}$ \\
\hline $\begin{array}{l}-\infty \\
-\infty \\
= \\
-\infty\end{array}$ & $\begin{array}{l}\text { ND } \\
\text { ND } \\
- \\
\text { ND } \\
\text { ND }\end{array}$ & $\begin{array}{l}40 \\
50 \\
-0 \\
20 \\
100\end{array}$ & $\begin{array}{r}9999 \\
9999 \\
-- \\
9999 \\
9999\end{array}$ & $\begin{array}{l}18 \\
18 \\
18 \\
18 \\
16\end{array}$ \\
\hline $\begin{array}{l}-\infty \\
-\infty \\
-\infty \\
-\infty\end{array}$ & $\begin{array}{l}-\infty \\
-\infty \\
30 \\
30\end{array}$ & $\begin{array}{l}-\infty \\
-\infty \\
20 \\
40\end{array}$ & $\begin{array}{l}9999 \\
9999 \\
9999 \\
9999 \\
9999\end{array}$ & $\begin{array}{l}16 \\
16 \\
11 \\
102 \\
102\end{array}$ \\
\hline $\begin{array}{l}-\infty \\
-\infty \\
-\infty \\
-\infty\end{array}$ & $\begin{array}{l}20 \\
-0 \\
\text { ND } \\
\text { NO } \\
10\end{array}$ & $\begin{array}{l}\text { NO } \\
-1 \\
20 \\
40 \\
\text { ND }\end{array}$ & $\begin{array}{r}9999 \\
-0 \\
9999 \\
9999 \\
9999\end{array}$ & $\begin{array}{r}102 \\
102 \\
102 \\
23 \\
23\end{array}$ \\
\hline $\begin{array}{l}- \\
-\infty \\
-\infty \\
-\infty\end{array}$ & $\begin{array}{l}20 \\
-0 \\
\text { ND } \\
10 \\
10\end{array}$ & $\begin{array}{l}20 \\
-0 \\
40 \\
30 \\
30\end{array}$ & $\begin{array}{r}9999 \\
-0 \\
9999 \\
9999 \\
9999\end{array}$ & $\begin{array}{l}23 \\
23 \\
23 \\
23 \\
28\end{array}$ \\
\hline $\begin{array}{l}-\infty \\
-\infty \\
-\infty \\
-\infty\end{array}$ & $\begin{array}{r}10 \\
00 \\
20 \\
800 \\
-\infty\end{array}$ & $\begin{array}{l}\text { ND } \\
30 \\
80 \\
-\infty\end{array}$ & $\begin{array}{r}9999 \\
9999 \\
9999 \\
9999 \\
-\end{array}$ & $\begin{array}{l}28 \\
28 \\
28 \\
28 \\
28\end{array}$ \\
\hline $\begin{array}{l}-\infty \\
\text { ND } \\
=- \\
=-\end{array}$ & $\begin{array}{l}\text { ND } \\
-- \\
\text { ND } \\
30 \\
--\end{array}$ & $\begin{array}{r}100 \\
160 \\
\text { ND } \\
\ldots\end{array}$ & $\begin{array}{r}9999 \\
=0 \\
9999 \\
9999 \\
9999\end{array}$ & $\begin{array}{l}28 \\
28 \\
26 \\
26 \\
26\end{array}$ \\
\hline
\end{tabular}


Table 5.--Chemical analyses of

LOCAL

IDENT-

I-

FIER

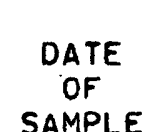

TOTAL

DEPTH

DIS-

DIS-

WELL

SOLVED

SILICA

SOLVEO

SAMPLE

(FT)

(SIO2)

IRON

(FE)

$(M G / L) \quad(U G / L)$

SC00406532ADA
SC00406532ADD

$S C 00406532 B A B$

SC00406532CBC

SC00406532DBB2

SC0040653208B3

SC00406533BAB I

SC00406533BAB2

SC00406533BAB3

SC00406533CBC

$\begin{array}{rr}75-08-18 & 26 \\ 75-12-07 & 26 \\ 75-12-09 & 26 \\ 74-11-25 & 20 \\ 75-08-18 & 20 \\ & \\ 75-12-07 & 20 \\ 75-08-18 & 11 \\ 74-11-26 & 16 \\ 75-12-07 & 16 \\ 75-06-18 & 151 \\ & \\ 75-06-18 & 248 \\ 75-08-20 & 248 \\ 75-12-08 & 248 \\ 75-12-08 & 248 \\ 75-06-18 & 28\end{array}$

75-08-19

$75-12-08$

$75-12-08$

76-04-02

$75-06-18$

75-08-19

75-12-08

75-12-08

76-04-02

$74-11-26$

75-02-12

75-02-12

75-08-18

$75-12-08$

$75-12-08$

76-04-02

$74-10-11$

$74-11-25$

$74-11-27$

75-02-12

75-05-05

$75-08-18$

75-12-09

75-12-09

76-04-05
26

26

26

20

20

20

11

16

151

248

248

248

28

28

28

28

28

82

82

82

82

82

22

22

22

22

22

22

22

20

20

20

20

20

20

20

20

20
$\Longrightarrow \quad 650$

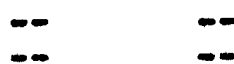

-
-80

$--\quad 340$

- $\quad-$

$=90$

- 30

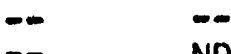

ND

-- ND

$-\infty$

14

30

-. ND

-- 60

-- 40

-- $\quad$--

-- NO

$-\quad$ ND

- 20

- 50

$-\infty \quad-$

-- 20

$--\quad 40$

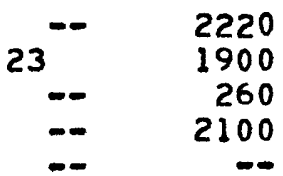

--

$-0910$

$\therefore \quad 30$

--

-- 150

-- 60

-- 40

-- 550

-- $\quad 0$

-. 110 
water from welzs--Continued

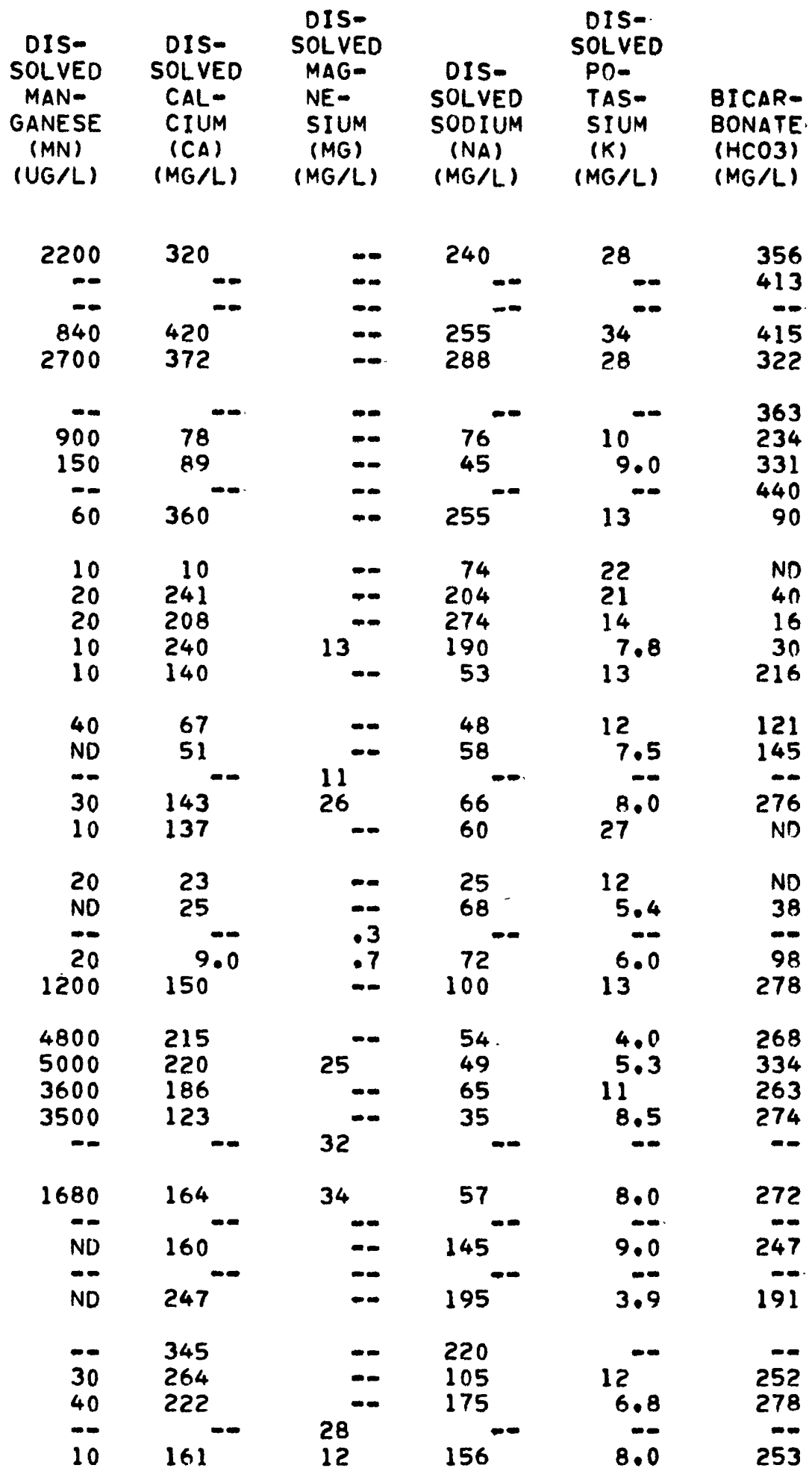




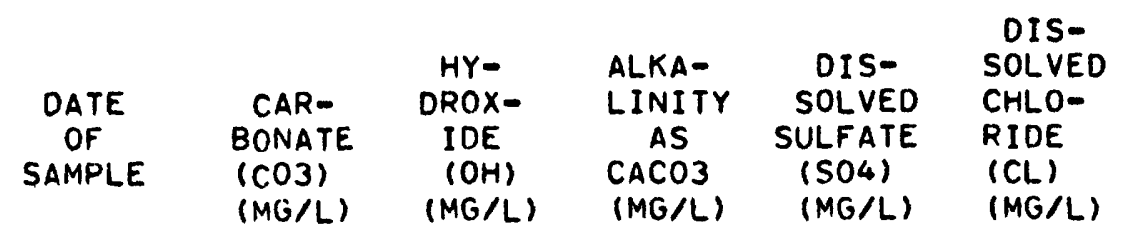

75-08-18

75-12-07

NO

ND

75-12-09

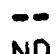

$75-08-18$

ND

75-12-07

$75-08-18$

$74-11-26$

$75-12-07$

$75-06-18$

ND

ND

ND

ND

ND

NO

$75-06-18$

$75-08-20$

28

$75-12-08$

$75-12-08$

75-06-18

NO

24

4

NO

75-08-19

75-12-08

$75-12-08$

76-04-02

75-06-18

160

16

--

ND

75-08-19

$75-12-08$

$75-12-08$

76-04-02

$74-11-26$

78

ND

$\begin{array}{lll}292 & 1220 & 50 \\ 339 & & \\ -- & 1320^{--} & 42 \\ 340 & 2^{--} & 29 \\ 264 & 1900^{--} & 35\end{array}$

75-02-12

$75-02-12$

$75-08-18$

$75-12-08$

75-12-08

80

114

$--$

78

NO

298

ND

ND

ND

ND

192

271

361

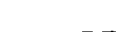

35

76-04-02

$74-10-11$

$74-11-25$

$74-11-27$

75-02-12

150

74

$720^{-0}$

$23^{-}$

25

D

$-$

790

$33 \quad 790$

$\begin{array}{ll}53 & 840\end{array}$

ND

31

177

440

$418^{-}$

ND

366

ND

146

$\begin{array}{ll}360^{-\infty} & 36 \\ 876^{-0} & 32 \\ \text { ND } & 21\end{array}$

226

645

--

141

221

ND

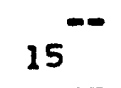

-

ND

210

228

ND

189

180

180

180

$-$

220

274

216

225

-

27

ND

ND

ND

$420^{-}$

555

660

$-$

ND

223

628

203

628

$\begin{array}{r}38 \\ -\quad 134 \\ \hline\end{array}$

ND

-

203

$-$

157

$-$

132

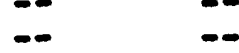

75-05-05

75-08-18

75-12-09

75-12-09

76-04-05

NO

207
228
208

$840^{--}$
$840^{--}$
$857^{--}$

116

--
ND
ND
--

23

25

26

32

39

36

37

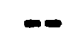


DIS-

SOLVED

FLUO-

RIDE

(F)

DIS-

DIS.

SOLVED TOTAL SOLVED SOLVED NITRATE NITRATE

SOLVED SOLVED
NITRATE NITRITE

(N)

(NO3)

(NO3)

(N)

$(M G / L) \quad(M G / L)$

$\begin{array}{cc} & \text { OIS } \\ \text { TOTAL' } & \text { SOLVED } \\ \text { NITRITE } & \text { NITRITE } \\ \text { (NOL) } & \text { (NOL) } \\ \text { (MG/L) } & (M G / L)\end{array}$

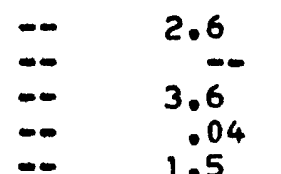

$=$

$11 \ldots$

15

$\ldots \quad 1.5$

6.18

.01

.02

$\begin{array}{lr}-0 & .05 \\ = & .08 \\ = & .08 \\ -0 & .16\end{array}$

$\therefore \quad 2.2$

$-0.02$

$-\infty$

$-$

. .6
.10

.04

$\therefore \quad .01$

$\ldots$

.04
$\ldots$
$-\infty$

$\begin{array}{ll}\therefore & \square \\ \therefore & \text { ND } \\ \therefore & -5 \\ -\therefore & \text { ND }\end{array}$

$\begin{array}{ll}-\therefore & 15 \\ \therefore & \text { ND } \\ \therefore & \text { ND }\end{array}$

.03

$-\infty$

$=01$

.00

.01

.00

ND

.06

$\begin{array}{ll}\therefore & \quad 15 \\ \therefore & \text { ND } \\ \therefore & -\infty\end{array}$

$=\quad .07$

$\infty$

3.8

.10

$\begin{array}{ll}-\infty & 3.2 \\ -\infty & 3.3\end{array}$

$-\infty$

5.5

.03

.00

$\ldots .01$

$-\infty$

$-0.00$

-01
-0

.00

$\begin{array}{ll}-\infty & \text { ND } \\ -\infty & .01\end{array}$

$\begin{array}{ll}-0 & 01 \\ -\infty & .01\end{array}$

$-$

$-$

.22

-0.00
$-0 \quad .00$

.02

.08

ND

$-$

$-$

.33

$\ldots .02$

- NO

$\ldots .02$

$-\infty$

$+$

.- .03

$-$

ND

$-\infty$

ND

$-$

$-$

-0.79
-0.39

ND

ND

.0 .18

$-\infty$

5.3

.16

$-\infty$

.71

Nn

$-\infty$

ND

$-.14$

$-$

.64

$-$

ND

$\begin{array}{ll}-0 & .06 \\ -0 & -0\end{array}$

$\begin{array}{ll}-0 & .28 \\ -0 & -0\end{array}$

$-$

$-$

$-\infty$

-- 21

$-\infty \quad 22$

$-\infty \quad 31$

$-\infty$

NO

$\begin{array}{ll}-0 & 4.9 \\ - & 5.2\end{array}$

$\ldots \quad 7.0$

-- 21

$\begin{array}{ll}-0 & 4.7 \\ - & 4.3\end{array}$

.00

.00

.06

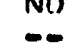

ND

ND

Nก

$=\quad 3.6$

$-\quad 15$

$=15$

$-$

$\begin{array}{ll}-0 & .01 \\ - & .01\end{array}$

$-.20$

$=\quad 3.6$

$\because$

Nn 
Table 5.--Chemical analyses of

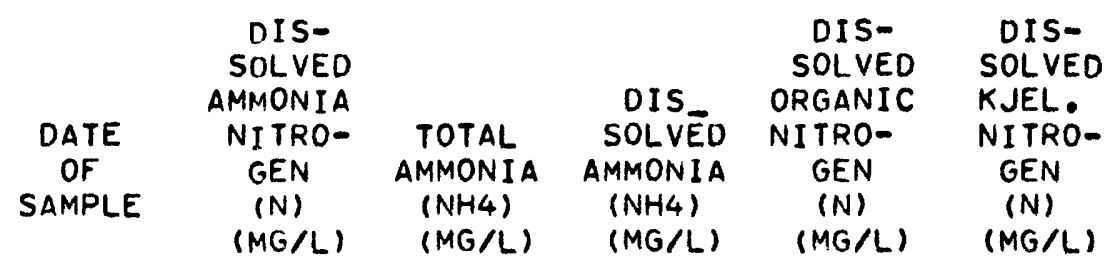

\begin{tabular}{|c|c|c|c|c|c|}
\hline $\begin{array}{l}75-08-18 \\
75-12-07 \\
75-12-09 \\
74-11-25 \\
75-08-18\end{array}$ & $\begin{array}{r}.50 \\
-- \\
\text { ND } \\
\text { ND } \\
.40\end{array}$ & $\begin{array}{l}-\infty \\
-\infty \\
-\infty \\
-\infty\end{array}$ & $\begin{array}{c}.64 \\
-- \\
-- \\
.5 \\
.52\end{array}$ & $\begin{array}{c}3.1 \\
= \\
= \\
1.0\end{array}$ & $\begin{array}{c}3.6 \\
-.0 \\
1.8 \\
5.9 \\
1.4\end{array}$ \\
\hline $\begin{array}{l}75-12-07 \\
75-08-18 \\
74-11-26 \\
75-12-07 \\
75-06-18\end{array}$ & $\begin{array}{r}-- \\
\text { ND } \\
\text { ND } \\
-- \\
.60\end{array}$ & $\begin{array}{l}-- \\
-- \\
-- \\
--\end{array}$ & $\begin{array}{l}-\infty \\
-0 \\
-77\end{array}$ & $\begin{array}{l}= \\
= \\
-\infty \\
-\infty\end{array}$ & $\begin{array}{r}-.5 \\
1.5 \\
1.6 \\
-5 \\
.50\end{array}$ \\
\hline $\begin{array}{l}75-06-18 \\
75-08-20 \\
75=12-08 \\
75-12-08 \\
75-06-18\end{array}$ & $\begin{array}{l}.60 \\
.30 \\
.20 \\
.27 \\
.40\end{array}$ & $\begin{array}{l}-\infty \\
- \\
-\infty \\
-\infty\end{array}$ & $\begin{array}{l}.77 \\
.39 \\
.26 \\
.35 \\
.52\end{array}$ & $\begin{array}{r}.0 \\
.40 \\
.40 \\
.31 \\
.00\end{array}$ & $\begin{array}{l}.20 \\
.70 \\
.60 \\
.58 \\
.40\end{array}$ \\
\hline $\begin{array}{l}75-08-19 \\
75-12-08 \\
75-12-08 \\
76-04-02 \\
75-06-18\end{array}$ & $\begin{array}{r}.20 \\
.10 \\
-0 \\
\text { ND } \\
.60\end{array}$ & $\begin{array}{l}= \\
=- \\
=- \\
--\end{array}$ & $\begin{array}{r}.26 \\
.13 \\
-- \\
-- \\
.77\end{array}$ & $\begin{array}{l}-0 \\
-00 \\
-\infty \\
-\infty\end{array}$ & $\begin{array}{r}.0 \\
.10 \\
.60 \\
.60\end{array}$ \\
\hline $\begin{array}{l}75-08-19 \\
75-12-08 \\
75-12-08 \\
76-04-02 \\
74-11-26\end{array}$ & $\begin{array}{r}.10 \\
110 \\
-10 \\
10 \\
\text { ND }\end{array}$ & $\begin{array}{l}-- \\
-- \\
-\infty\end{array}$ & $\begin{array}{r}.13 \\
.13 \\
-13 \\
.13 \\
--\end{array}$ & $\begin{array}{r}.40 \\
.90 \\
-0\end{array}$ & $\begin{array}{r}.50 \\
0 \\
1.0 \\
.80\end{array}$ \\
\hline $\begin{array}{l}75-02-12 \\
75-02-12 \\
75-08-18 \\
75-12-08 \\
75-12-08\end{array}$ & $\begin{array}{l}.20 \\
.25 \\
.40 \\
.20 \\
.-\end{array}$ & $\begin{array}{l}-- \\
- \\
-\end{array}$ & $\begin{array}{r}.26 \\
.32 \\
.52 \\
.26 \\
.--\end{array}$ & $\begin{array}{r}.70 \\
.36 \\
.20 \\
.20 \\
--\end{array}$ & $\begin{array}{l}.90 \\
.61 \\
.60 \\
.40 \\
.0\end{array}$ \\
\hline $\begin{array}{l}76-04-02 \\
74-10-11 \\
74-11-25 \\
74-11-27 \\
75-02-12\end{array}$ & $\begin{array}{l}\text { NO } \\
-- \\
\text { NO } \\
-\infty \\
\text { NO }\end{array}$ & $\begin{array}{l}-\infty \\
-\infty \\
-\infty \\
-\infty\end{array}$ & $\begin{array}{l}-- \\
=- \\
=- \\
=-\end{array}$ & $\begin{array}{l}- \\
- \\
- \\
-\end{array}$ & $\begin{array}{r}.10 \\
-20 \\
.20 \\
-30\end{array}$ \\
\hline $\begin{array}{l}75-05-05 \\
75-08-18 \\
75-12-09 \\
75-12-09 \\
76-04-05\end{array}$ & $\begin{array}{l}.20 \\
\text { ND } \\
\text { ND } \\
-- \\
\text { NO }\end{array}$ & $=$ & $\begin{array}{l}.26 \\
-- \\
=- \\
=-\end{array}$ & $\begin{array}{r}7.4 \\
=- \\
=- \\
=-\end{array}$ & $\begin{array}{c}7.6 \\
.20 \\
.30 \\
.30\end{array}$ \\
\hline
\end{tabular}




\begin{tabular}{|c|c|c|c|c|c|c|}
\hline $\begin{array}{l}\text { DIS- } \\
\text { SOLVED } \\
\text { ORTHO. } \\
\text { PHOS- } \\
\text { PHORUS } \\
\text { (P) } \\
\text { (MG/L) }\end{array}$ & $\begin{array}{l}\text { DIS- } \\
\text { SOLVED } \\
\text { ORTHO } \\
\text { PHOS- } \\
\text { PHATE } \\
\text { (PO4) } \\
\text { (MG/L) }\end{array}$ & $\begin{array}{l}\text { DIS- } \\
\text { SOLVED } \\
\text { SOLIDS } \\
\text { (RESI- } \\
\text { DUE AT } \\
\left.180^{\circ} \mathrm{C}\right) \\
(\mathrm{MG} / \mathrm{L})\end{array}$ & $\begin{array}{l}\text { DIS- } \\
\text { SOLVED } \\
\text { SOLIDS } \\
\text { (RESI- } \\
\text { DUE AT } \\
\left.105^{\circ} \mathrm{C}\right) \\
\text { (MG/L) }\end{array}$ & $\begin{array}{l}\text { DIS- } \\
\text { SOLVED } \\
\text { SOLIDS } \\
\text { (SUM OF } \\
\text { CONSTI- } \\
\text { TUENTS) } \\
\text { (MG/L) }\end{array}$ & $\begin{array}{l}\text { HARD- } \\
\text { NESS } \\
(C A, M G) \\
(M G / L)\end{array}$ & $\begin{array}{l}\text { NON- } \\
\text { CAR- } \\
\text { BONATE } \\
\text { HARD- } \\
\text { NESS } \\
\text { (MG/L) }\end{array}$ \\
\hline ND & $-\infty$ & $-\infty$ & 2170 & $-\infty$ & 1020 & 730 \\
\hline$=$ & $-\infty$ & - & -- & $-\infty$ & $-\infty$ & $\ldots$ \\
\hline $3.1_{\text {ND }}^{\text {ND }}$ & $9.7^{--}$ & $=$ & $\begin{array}{l}2290 \\
3480 \\
3170\end{array}$ & $\ddot{m}$ & $\begin{array}{l}1070 \\
1690 \\
1380\end{array}$ & $\begin{array}{l}1400 \\
1100\end{array}$ \\
\hline$=-$ & - & $=$ & $-\infty$ & $-\infty$ & $-\infty$ & $-\infty$ \\
\hline $\begin{array}{l}.09 \\
.78\end{array}$ & $\begin{array}{l}.28 \\
2.4\end{array}$ & $=$ & $\begin{array}{l}598 \\
497\end{array}$ & $=$ & $\begin{array}{l}305 \\
400\end{array}$ & $\begin{array}{l}110 \\
130\end{array}$ \\
\hline.$\overline{11}$ & .34 & $=$ & $15 \overline{30}$ & $=$ & $6 \overline{3}$ & 610 \\
\hline $\begin{array}{l}\text { ND } \\
.01 \\
.02 \\
.01 \\
.17\end{array}$ & $\begin{array}{l}.0 \\
.03 \\
.06 \\
.03 \\
.52\end{array}$ & $\begin{array}{l}= \\
=- \\
=\end{array}$ & $\begin{array}{r}1660 \\
1510 \\
1550 \\
-0 \\
992\end{array}$ & $\begin{array}{r}=0 \\
=\infty \\
1400 \\
=-\infty\end{array}$ & $\begin{array}{l}794 \\
690 \\
674 \\
650 \\
546\end{array}$ & $\begin{array}{l}600 \\
620 \\
620 \\
370\end{array}$ \\
\hline $\begin{array}{r}\text { ND } \\
.02\end{array}$ & .06 & $=$ & $\begin{array}{l}456 \\
638\end{array}$ & $-\infty$ & $\begin{array}{l}218 \\
322\end{array}$ & $\begin{array}{r}0 \\
180\end{array}$ \\
\hline$-\infty$ & $-\infty$ & - & - & - & -- & - \\
\hline $\begin{array}{r}\text { ND } \\
.01\end{array}$ & .03 & $\therefore$ & $\begin{array}{r}1060 \\
742\end{array}$ & $=$ & $\begin{array}{l}622 \\
484\end{array}$ & 400 \\
\hline $\begin{array}{r}\text { ND } \\
.04\end{array}$ &.$\overline{12}$ & $=$ & $\begin{array}{l}285 \\
239\end{array}$ & $=$ & $\begin{array}{l}50 \\
37\end{array}$ & -- \\
\hline$-\infty$ & -- & $-\infty$ & -- & $\infty$ & $-\infty$ & $-\infty$ \\
\hline $\begin{array}{l}.02 \\
.48\end{array}$ & 1.06 & 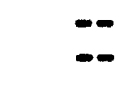 & $\begin{array}{l}263 \\
923\end{array}$ & 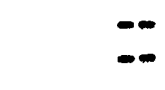 & $\begin{array}{r}32 \\
627\end{array}$ & $\begin{array}{r}0 \\
400\end{array}$ \\
\hline $\begin{array}{l}1.3 \\
.04 \\
.02 \\
.04\end{array}$ & $\begin{array}{l}4.1 \\
.12 \\
.06 \\
.12\end{array}$ & $\begin{array}{l}= \\
-\infty \\
-\infty\end{array}$ & $\begin{array}{r}1080 \\
=- \\
1260 \\
1250\end{array}$ & $\begin{array}{r}959 \\
-0 \\
=-\end{array}$ & $\begin{array}{l}650 \\
650 \\
761 \\
797\end{array}$ & $\begin{array}{l}430 \\
380 \\
550 \\
570\end{array}$ \\
\hline-- & -- & - & $-\infty$ & - & $-\infty$ & $\infty$ \\
\hline ND & $-\infty$ & - & 1270 & $-\infty$ & 789 & 570 \\
\hline-- & $=-$ & & 1500 & - & $-\infty$ & - \\
\hline .18 & .55 & $-\infty$ & 1610 & -- & 846 & 640 \\
\hline .60 & $1.8^{--}$ & $=$ & 1700 & $=$ & 729 & $5 \overline{70}$ \\
\hline $\begin{array}{r}.05 \\
.02\end{array}$ & $\begin{array}{l}.15 \\
.15 \\
.06\end{array}$ & $\ddot{-\infty}$ & $\begin{array}{l}1930 \\
1790 \\
1610\end{array}$ & $=$ & $\begin{array}{l}871 \\
749\end{array}$ & $\begin{array}{l}-0 \\
660 \\
520\end{array}$ \\
\hline$\overline{\text { ND }}$ & $-\infty$ & 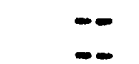 & $16 \overline{70}$ & $=$ & 803 & 600 \\
\hline ND & $-\infty$ & -- & 1670 & -- & 803 & 600 \\
\hline
\end{tabular}




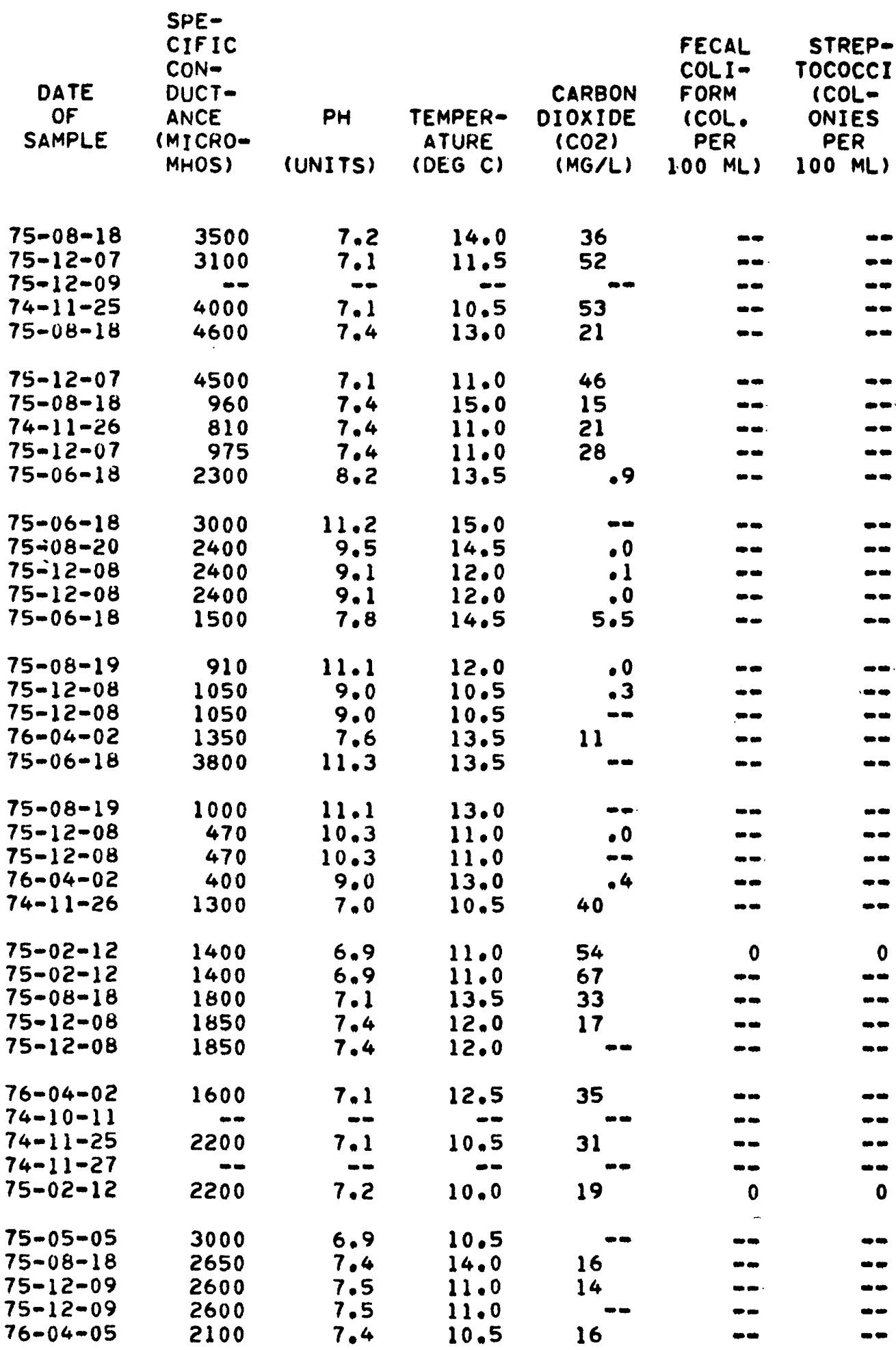


water from wells--Continued

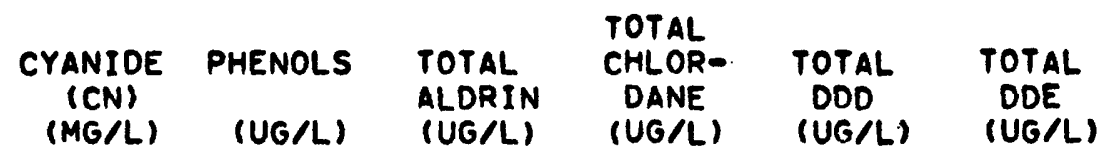

\begin{tabular}{|c|c|c|c|c|c|}
\hline$\infty$ & $-\infty$ & $-\infty$ & $\infty$ & $\infty$ & $-\infty$ \\
\hline$\infty$ & $\infty$ & $\infty$ & $\infty$ & $\infty$ & $\infty$ \\
\hline$\infty$ & $\infty$ & $\infty$ & $-\infty$ & $-\infty$ & $-\infty$ \\
\hline$-\infty$ & $-\infty$ & $\infty$ & $\infty$ & $\infty$ & $\infty$ \\
\hline$-\infty$ & $-\infty$ & $\infty$ & $\infty$ & $\cdots$ & $\infty$ \\
\hline$\infty$ & $\infty$ & $-\infty$ & $\infty$ & $-\infty$ & $-\infty$ \\
\hline- & $\infty$ & $-\infty$ & $\infty$ & $\infty$ & $-\infty$ \\
\hline & $-\infty$ & $-\infty$ & - & $-\infty$ & $\infty$ \\
\hline & $-\infty$ & $-\infty$ & $-\infty$ & $-\infty$ & $\infty$ \\
\hline 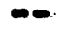 & $\infty$ & $-\infty$ & $\infty$ & $\infty$ & $\infty$ \\
\hline- & $\infty$ & $\infty$ & $\infty$ & $\rightarrow$ & \\
\hline & $\infty$ & -- & $-\infty$ & $-\infty$ & - \\
\hline & $-\infty$ & $-\infty$ & $\infty$ & $-\infty$ & $\infty$ \\
\hline- & $\infty$ & $\infty$ & $\infty$ & $\infty$ & $\infty$ \\
\hline 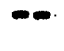 & - & $-\infty$ & $\infty$ & $\infty$ & $\infty$ \\
\hline & $-\infty$ & $\cdots$ & $\infty$ & $\infty$ & \\
\hline & $\infty$ & $\infty$ & $-\infty$ & - & \\
\hline & $-\infty$ & $\infty$ & $\infty$ & $\infty$ & 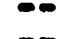 \\
\hline & $-\infty$ & - & $-\infty$ & $-\infty$ & $-\infty$ \\
\hline & $\cdots$ & - & $-\infty$ & $\infty$ & \\
\hline$\infty$ & $\infty$ & $\ldots$ & $\infty$ & 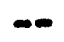 & \\
\hline & $-\infty$ & $\rightarrow$ & $-\infty$ & $\infty$ & \\
\hline & $-\infty$ & $-\infty$ & $-\infty$ & $\infty$ & \\
\hline & $\infty$ & $-\infty$ & $\infty$ & $\infty$ & \\
\hline & - & - & $\infty$ & - & \\
\hline & $-\infty$ & $\infty$ & - & $\infty$ & - \\
\hline & $-\infty$ & $\infty$ & $-\infty$ & $\cdots$ & \\
\hline & $\infty$ & $\infty$ & $-\infty$ & $\infty$ & \\
\hline & $\infty$ & $-\infty$ & $-\infty$ & $-\infty$ & \\
\hline & $-\infty$ & $-\infty$ & $-\infty$ & $-\infty$ & \\
\hline & $\infty$ & $-\infty$ & - & $-\infty$ & \\
\hline & $\infty$ & $-\infty$ & $\infty$ & $\infty$ & \\
\hline & $\infty$ & $-\infty$ & $\infty$ & $\infty$ & \\
\hline & $-\infty$ & $\infty$ & $-\infty$ & $\infty$ & \\
\hline & - & $\infty$ & - & $-\infty$ & \\
\hline & $\infty$ & $\infty$ & $\infty$ & $\infty$ & $\infty$ \\
\hline & $-\infty$ & No & NO & No & ND \\
\hline & -- & $-\infty$ & $-\infty$ & - & \\
\hline & 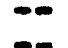 & - & $\infty$ & $\infty$ & \\
\hline & - & $-\infty$ & $\infty$ & $-\infty$ & \\
\hline
\end{tabular}


Table 5.--Chemical analyses of

\begin{tabular}{|c|c|c|c|c|c|c|}
\hline $\begin{array}{c}\text { DATE } \\
\text { OF } \\
\text { SAMPLE }\end{array}$ & $\begin{array}{l}\text { TOTAL } \\
\text { DDT } \\
\text { (UG/L) }\end{array}$ & $\begin{array}{l}\text { TOTAL } \\
\text { DI- } \\
\text { AZINON } \\
\text { (UG/L) }\end{array}$ & $\begin{array}{l}\text { TOTAL } \\
\text { DI- } \\
\text { ELDRIN } \\
\text { (UG/L) }\end{array}$ & $\begin{array}{l}\text { TOTAL } \\
\text { ENDRIN } \\
\text { (UG/L) }\end{array}$ & $\begin{array}{l}\text { TOTAL } \\
\text { HEPTA- } \\
\text { CHLOR } \\
\text { (UG/L) }\end{array}$ & $\begin{array}{l}\text { HEPTA- } \\
\text { CHLOR } \\
\text { EPOXIDE } \\
\text { (UG/L) }\end{array}$ \\
\hline
\end{tabular}

$75-08-18$

$75-12-07$

75-12-09

$74-11-25$

$75-08-18$

$75-12-07$

$75-08-18$

$74-11-26$

$75-12-07$

$75-06-18$

$75-06-18$

$75 \div 08-20$

$75 \div 12-08$

$75-12-08$

$75-06-18$

$75-08-19$

$75-12-08$

$75-12-08$

$76-04-02$

75-06-18

75-08-19

$75-12-08$

$75-12-08$

76-04-02

74-11-26

$75-02-12$

$75-02-12$

$75-08-18$

$75-12-08$

$75-12-08$

76-04-02

$74-10-11$

$74-11-25$

$74-11-27$

75-02-12

$75-05-05$

$75-08-18$

75-12-09

75-12-09

76-04-05

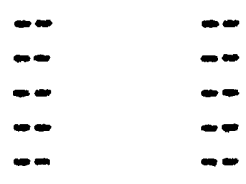

$-\infty$

$-$

$-$

$-$

$-$

$-$

$-$

--

$-$

$-$

$-$

$-\infty$

$-\infty$

$-\infty$

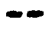

$-$

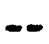

$\because$

$-$

--

$\because$

$\because$

$\because$

$-$

$-$

$-$

$\because$

$-$

$-$

ND

$\overline{--}$

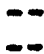

$-\infty$
$-\infty$
$-\infty$

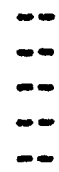

$-\infty$

$-$

$-$

$-$

$-$

$-0$

$-\infty$

$-\infty$

$-\infty$

$-$

$-$

$-$

$-\infty$

$-\infty$

$-\infty$

$-\infty$

$=$

$-$

$\ddot{-}$

$\because$

$\because$

$\because$

$-$

$\because$

--

-

$-$

$-$

ND

$-$

$-$

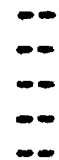

$-\infty$
$-\infty$
$-\infty$
$-\infty$

$-\infty$
$-\infty$
$-\infty$
$-\infty$

$\because$

$\because$

$\because$

$-$

$-$

$-\infty$

$-\infty$

$-\infty$

$-$

$-\infty$

$-$

$--$

--

$-$

$-$

$\because$

$-\infty$

$-$

$-\infty$

$-\infty$

$-\infty$

$-$

$-\infty$

$-\infty$

$-$

$-$

$-\infty$

ND

ND

$=$

$-\infty$

$-$

$-$ $-\infty$
$-\infty$
$-\infty$
$-\infty$
$-\infty$
$=\infty$
$-\infty$
$-\infty$

$-$

$-\infty$

-

$-$

$-\infty$

$-$

$=$

$-\infty$

$-$

$-$

$-$

$-$

$-\infty$

$-$

-.

$-$

$-\infty$

$-$

$-$

$=$

$-$

$=$

$-$

$-$

$-$

$-$

ND

$-$

$-$ 
water from wells--Continued

$\begin{array}{llllll} & \text { TOTAL } & \text { TOTAL } & & & \\ \text { METHYL } & \text { TOTAL } & & \text { TOTAL } \\ \text { TOTAL } & \text { MALA- } & \text { PARA- } & \text { PARA- } & \text { TOTAL } & \text { TOX- } \\ \text { LINDANE } & \text { THION } & \text { THION } & \text { THION } & \text { PCB } & \text { APHENE } \\ (U G / L) & (U G / L) & (U G / L) & (U G / L) & (U G / L) & (U G / L)\end{array}$

\begin{tabular}{|c|c|c|c|c|c|}
\hline- & $-\infty$ & $\infty$ & $m$ & $\infty$ & 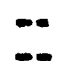 \\
\hline$=$ & $-\infty$ & $-\infty$ & - & $-\infty$ & $=$ \\
\hline$=$ & 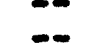 & $=-$ & $-\infty$ & $-\infty$ & $=$ \\
\hline- & $=$ & $-\infty$ & $\ldots$ & -2 & - \\
\hline & & & & & \\
\hline$-\infty$ & $\cdots$ & $-\infty$ & $-\infty$ & $-\infty$ & - \\
\hline$=$ & $-\infty$ & $-\infty$ & $\infty$ & $-\infty$ & \\
\hline$-\infty$ & $-\infty$ & $-\infty$ & $\infty$ & $-\infty$ & \\
\hline$-\infty$ & $\infty$ & $-\infty$ & $-\infty$ & $\infty$ & 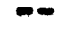 \\
\hline- & $\cdots$ & $\infty$ & $-\infty$ & $\infty$ & 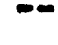 \\
\hline- & - & $\infty$ & $-\infty$ & $-\infty$ & " \\
\hline$\infty$ & $-\infty$ & -- & $\infty$ & $-\infty$ & 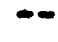 \\
\hline$=$ & $\infty$ & $\infty$ & $\infty$ & $-\infty$ & \\
\hline$-\infty$ & $\cdots$ & $\cdots$ & $-\infty$ & $\infty$ & \\
\hline$-\infty$ & -- & $-\infty$ & -- & $-\infty$ & * \\
\hline- & $-\infty$ & $\infty$ & $-\infty$ & $-\infty$ & \\
\hline$-\infty$ & $\cdots$ & $\cdots$ & $\infty$ & -- & \\
\hline$-\infty$ & $\cdots$ & $\cdots$ & $\infty$ & $\cdots$ & \\
\hline$=$ & $-\infty$ & - & $\infty$ & - & \\
\hline$-\infty$ & $-\infty$ & - & $\infty$ & $-\infty$ & - \\
\hline$-\infty$ & $\infty$ & -- & -- & $-\infty$ & \\
\hline- & $\cdots$ & - & $\infty$ & $\cdots$ & \\
\hline- & $\cdots$ & - & $-\infty$ & $-\infty$ & \\
\hline$-\infty$ & - & $-\infty$ & $=$ & $=$ & \\
\hline$-\infty$ & - & $\infty$ & - & $\infty$ & \\
\hline-- & $-\infty$ & $-\infty$ & $-\infty$ & $-\infty$ & \\
\hline- & -- & -- & $-\infty$ & $-\infty$ & \\
\hline$-\infty$ & $\cdots$ & $-\infty$ & $-\infty$ & $-\infty$ & \\
\hline$=-$ & $-\infty$ & $-\infty$ & $-\infty$ & $-\infty$ & \\
\hline- & $-\infty$ & $\cdots$ & $\cdots$ & $\cdots$ & \\
\hline- & $-\infty$ & $-\infty$ & -- & $-\infty$ & \\
\hline-- & $-\infty$ & - & $-\infty$ & $-\infty$ & \\
\hline 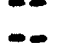 & $=$ & $-\infty$ & $=$ & $-\infty$ & \\
\hline$\cdots$ & $-\infty$ & $=$ & $=-$ & $=$ & \\
\hline & & & & 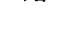 & \\
\hline ND & $-\infty$ & $-\overline{0}$ & $\ddot{-\infty}$ & $-\infty$ & \\
\hline- & 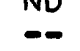 & $\begin{array}{l}\text { ND } \\
--\end{array}$ & ND & NO & \\
\hline & - & - & $-\infty$ & $-\infty$ & \\
\hline & - & - & $-\infty$ & $-\infty$ & \\
\hline
\end{tabular}




\begin{tabular}{|c|c|c|c|c|}
\hline $\begin{array}{l}\text { DATE } \\
\text { OF } \\
\text { SAMPLE }\end{array}$ & $\begin{array}{l}\text { DIS- } \\
\text { SOLVED } \\
\text { CAD- } \\
\text { MIUM } \\
\text { (CD) } \\
\text { (UG/L) }\end{array}$ & $\begin{array}{l}\text { DIS- } \\
\text { SOLVED } \\
\text { CHRO- } \\
\text { MIUM } \\
\text { (CR) } \\
\text { (UG/L) }\end{array}$ & $\begin{array}{l}\text { DIS- } \\
\text { SOLVED } \\
\text { COPPER } \\
\text { (CU) } \\
\text { (UG/L) }\end{array}$ & $\begin{array}{l}\text { DIS- } \\
\text { SOLVED } \\
\text { LEAD } \\
\text { (PB) } \\
(U G / L)\end{array}$ \\
\hline $\begin{array}{l}75-08-18 \\
75-12-07 \\
75-12-09 \\
74-11-25 \\
75-08-18\end{array}$ & $\begin{array}{r}2 \\
-- \\
-- \\
1 \\
5\end{array}$ & $\begin{array}{l}10 \\
-- \\
-- \\
70 \\
30\end{array}$ & $\begin{array}{l}20 \\
-\infty \\
N D \\
60\end{array}$ & $\begin{array}{l}10 \\
8- \\
80 \\
10\end{array}$ \\
\hline $\begin{array}{l}75-12-07 \\
75-08-18 \\
74-11-26 \\
75-12-07 \\
75-06-18\end{array}$ & $\begin{array}{r}-- \\
-1 \\
-\frac{1}{4}\end{array}$ & $\begin{array}{l}-- \\
\text { NO } \\
10 \\
--\end{array}$ & $\begin{array}{l}-\infty \\
40 \\
\text { ND } \\
-\infty \\
20\end{array}$ & $\begin{array}{l}-- \\
10 \\
20 \\
10\end{array}$ \\
\hline $\begin{array}{l}75-06-18 \\
75-08-20 \\
75-12-08 \\
75-12-08 \\
75-06-18\end{array}$ & $\begin{array}{r}\text { No } \\
1 \\
7 \\
-- \\
9\end{array}$ & $\begin{array}{l}10 \\
10 \\
\text { ND } \\
- \\
\text { ND }\end{array}$ & $\begin{array}{l}20 \\
40 \\
\text { ND } \\
-- \\
1\end{array}$ & $\begin{array}{r}30 \\
20 \\
100 \\
-- \\
10\end{array}$ \\
\hline $\begin{array}{l}75-08-19 \\
75-12-08 \\
75-12-08 \\
76-04-02 \\
75-06-18\end{array}$ & $\begin{array}{r}\text { ND } \\
2 \\
-- \\
3 \\
\text { ND }\end{array}$ & $\begin{array}{l}10 \\
\text { ND } \\
-- \\
10 \\
\text { ND }\end{array}$ & $\begin{array}{l}40 \\
\text { ND } \\
-0 \\
80 \\
20\end{array}$ & $\begin{array}{l}30 \\
20 \\
-- \\
50 \\
20\end{array}$ \\
\hline $\begin{array}{l}75-08-19 \\
75-12-08 \\
75-12-08 \\
76-04-02 \\
74-11-26\end{array}$ & $\begin{array}{r}\text { ND } \\
4 \\
-- \\
\text { ND } \\
\text { ND }\end{array}$ & $\begin{array}{l}\text { ND } \\
\text { NO } \\
-- \\
\text { ND } \\
10\end{array}$ & $\begin{array}{l}20 \\
30 \\
=- \\
20 \\
\text { ND }\end{array}$ & $\begin{array}{l}\text { ND } \\
50 \\
-- \\
\text { ND } \\
20\end{array}$ \\
\hline $\begin{array}{l}75-02-12 \\
75-02-12 \\
75-08-18 \\
75-12-08 \\
75-12-08\end{array}$ & $\begin{array}{r}2 \\
-- \\
2 \\
5 \\
--\end{array}$ & $\begin{array}{l}20 \\
10 \\
\text { ND } \\
-\infty\end{array}$ & $\begin{array}{l}10 \\
-\infty \\
30 \\
\text { ND } \\
-\infty\end{array}$ & $\begin{array}{l}40 \\
-- \\
20 \\
30 \\
--\end{array}$ \\
\hline $\begin{array}{l}76-04-02 \\
74-10-11 \\
74-11-25 \\
74-11-27 \\
75-02-12\end{array}$ & $\frac{-3}{3}$ & $\begin{array}{l}10 \\
50 \\
10\end{array}$ & $\begin{array}{l}20 \\
-2 \\
\text { ND } \\
-\infty \\
10\end{array}$ & $\begin{array}{l}30 \\
-- \\
\text { ND } \\
--\end{array}$ \\
\hline $\begin{array}{l}75-05-05 \\
75-08-18 \\
75-12-09 \\
75-12-09 \\
76-04-05\end{array}$ & $\begin{array}{r}-- \\
2 \\
5 \\
-\infty\end{array}$ & $\begin{array}{l}-\infty \\
\text { NO } \\
\text { NO }\end{array}$ & $\begin{array}{r}280 \\
20 \\
- \\
10\end{array}$ & $\begin{array}{l}-- \\
10 \\
70 \\
-- \\
\text { ND }\end{array}$ \\
\hline
\end{tabular}


water from wells--Continued

\begin{tabular}{|c|c|c|c|c|}
\hline $\begin{array}{c}\text { DIS- } \\
\text { SOLVED } \\
\text { MERCURY } \\
\text { (HG) } \\
\text { (UG/L) }\end{array}$ & $\begin{array}{l}\text { DIS- } \\
\text { SOLVED } \\
\text { NICKEL } \\
\text { (NI) } \\
(U G / L)\end{array}$ & $\begin{array}{l}\text { DIS- } \\
\text { SOLVED } \\
\text { ZINC } \\
\text { (ZN) } \\
\text { (UG/L) }\end{array}$ & $\begin{array}{l}\text { CODE } \\
\text { FOR } \\
\text { AGENCY } \\
\text { ANA- } \\
\text { LYZING } \\
\text { SAMPLE }\end{array}$ & $\begin{array}{c}\text { TOTAL } \\
\text { DEPTH } \\
\text { OF } \\
\text { WELL } \\
\text { (FT) }\end{array}$ \\
\hline $\begin{array}{l}-\infty \\
-\infty \\
-\infty\end{array}$ & $\begin{array}{l}70 \\
-\infty \\
30 \\
30\end{array}$ & $\begin{array}{r}210 \\
-0 \\
110 \\
320\end{array}$ & $\begin{array}{l}9999 \\
9999 \\
9999 \\
9999 \\
9999\end{array}$ & $\begin{array}{l}26 \\
26 \\
26 \\
20 \\
20\end{array}$ \\
\hline $\begin{array}{l}-\infty \\
-\infty \\
-\infty\end{array}$ & $\begin{array}{l}-\infty \\
30 \\
10 \\
-- \\
20\end{array}$ & $\begin{array}{l}-10 \\
70 \\
-- \\
10\end{array}$ & $\begin{array}{l}9999 \\
9999 \\
9999 \\
9999 \\
9999\end{array}$ & $\begin{array}{l}20 \\
11 \\
16 \\
16 \\
151\end{array}$ \\
\hline $\begin{array}{l}-\infty \\
-\infty \\
-\infty \\
-\infty\end{array}$ & $\begin{array}{l}20 \\
50 \\
60 \\
-- \\
30\end{array}$ & $\begin{array}{r}30 \\
500 \\
\text { ND } \\
20 \\
20\end{array}$ & $\begin{array}{r}9999 \\
9999 \\
9999 \\
999\end{array}$ & $\begin{array}{r}248 \\
248 \\
248 \\
248 \\
28\end{array}$ \\
\hline $\begin{array}{l}-\infty \\
-\infty \\
-\infty \\
-\infty\end{array}$ & $\begin{array}{l}20 \\
\text { ND } \\
-- \\
\text { ND } \\
10\end{array}$ & $\begin{array}{r}20 \\
\text { ND } \\
-0 \\
100 \\
20\end{array}$ & $\begin{array}{r}9999 \\
9999 \\
99 \\
9999 \\
9999\end{array}$ & $\begin{array}{l}28 \\
28 \\
28 \\
28 \\
82\end{array}$ \\
\hline $\begin{array}{l}-\infty \\
-\infty \\
-\infty \\
\text { ND }\end{array}$ & $\begin{array}{l}30 \\
10 \\
-- \\
\text { ND } \\
\text { ND }\end{array}$ & $\begin{array}{l}20 \\
\text { ND } \\
-- \\
\text { ND } \\
80\end{array}$ & $\begin{array}{r}9999 \\
9999 \\
-- \\
9999 \\
9999\end{array}$ & $\begin{array}{l}82 \\
82 \\
82 \\
82 \\
22\end{array}$ \\
\hline $\begin{array}{l}-\infty \\
=- \\
-\infty \\
-\infty\end{array}$ & $\begin{array}{l}10 \\
-- \\
40 \\
N D \\
--\end{array}$ & $\begin{array}{l}\text { ND } \\
-\infty \\
40 \\
60 \\
-\infty\end{array}$ & $\begin{array}{r}9999 \\
-- \\
9999 \\
9999 \\
--\end{array}$ & $\begin{array}{l}22 \\
22 \\
22 \\
22 \\
22\end{array}$ \\
\hline$=$ & $\begin{array}{l}\text { ND } \\
-\infty \\
10 \\
-\infty\end{array}$ & $\begin{array}{r}110 \\
80 \\
260\end{array}$ & $\begin{array}{l}9999 \\
9999 \\
9999 \\
9999 \\
9999\end{array}$ & $\begin{array}{l}22 \\
20 \\
20 \\
20 \\
20\end{array}$ \\
\hline $\begin{array}{l}-\infty \\
-\infty \\
-\infty \\
-\infty\end{array}$ & $\begin{array}{l}-10 \\
\text { ND } \\
10\end{array}$ & $\begin{array}{r}130 \\
1800 \\
220\end{array}$ & $\begin{array}{r}9999 \\
9999 \\
9999 \\
-- \\
9999\end{array}$ & $\begin{array}{l}20 \\
20 \\
20 \\
20 \\
20\end{array}$ \\
\hline
\end{tabular}


Table 5.--Chemical analyses of

LOCAL
IDENT-
I-
FIER

$S C 00406534 C B B$

SC00506502BCB

SC00506502DCA

$S C 00506503 A B B$

SC00506504AAAZ

$S C 0050650480 B$

SC00506504CAB1

SC00506504CAB2

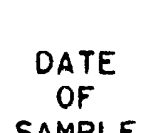

TOTAL DEPTH

OF

WELL

(FT)
DIS-

SOLVED

SILICA

(SI02)

(MG/L)
DIS-

SOLVED

IRON

(FE)

(UG/L)

$\begin{array}{ll}74-11-25 & 16 \\ 75-02-11 & 16 \\ 75-05-05 & 16 \\ 75-08-14 & 16 \\ 75-12-04 & 16\end{array}$

75-12-04 16

76-03-31 16

74-10-11 11

74-10-11 : 24

74-11-25 17

$74-11-25 \quad 17$

$75-02-10$

75-05-05

$75-08-14$

75-12-04

17

17

17

17

75-12-04

76-04-16

$74-11-25$

$75-02-11$

75-05-02

17

17

17

17

17

75-08-15

$75-08-15$

75-12-09

75-12-09

76-04-16

17

17

17

17

17

$74-11-25$

$75-02-10$

33

75-02-11

33

$75-05-02$

$75-08-15$

33

33

33

75-12-09

33

$75-12-09$

33

75-08-20

18

75-12-09

18

18

75-12-09 18

$75-06-19$

107

$75-08-20$

107

75-12-09

107

75-12-09

$\begin{array}{rr}- & 50 \\ - & 170 \\ - & 170 \\ - & 160 \\ - & 90\end{array}$

16

16

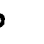

$-\infty$

.

..

$-$

$-\infty$

30

$-$

.

$\because$

$-$

20

$-$

$-$

60

520

590

420

710

$\begin{array}{rr}-- & - \\ -- & - \\ -- & 50 \\ -- & 1530 \\ - & 2900\end{array}$

- 2600

$\begin{array}{ll}-\infty & -\infty\end{array}$

$-1600$

$-$

$-$

--

3

33

3

$-\infty \quad 530$

$\because$

--

$\because$

30

$\cdots$

10

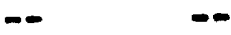

- 10

- 20

- 60

$-$ 
water from wells--Continued

\begin{tabular}{cccccc} 
DIS- & DIS- & DIS- & \multicolumn{3}{c}{ DIS- } \\
SOLVED & SOLVEO & \\
SOLVED & SOLVED & MAG- & OIS- & PO- & \\
MAN- & CAL- & NE- & SOLVED & TAS- & BICAR- \\
GANESE & CIUM & SIUM & SODIUM & SIUM & BONATE \\
(MN) & (CA) & (MG) & (NA) & (K) & (HCO3) \\
$(U G / L)$ & $(M G / L)$ & $(M G / L)$ & (MG/L) & $(M G / L)$ & $(M G / L)$
\end{tabular}

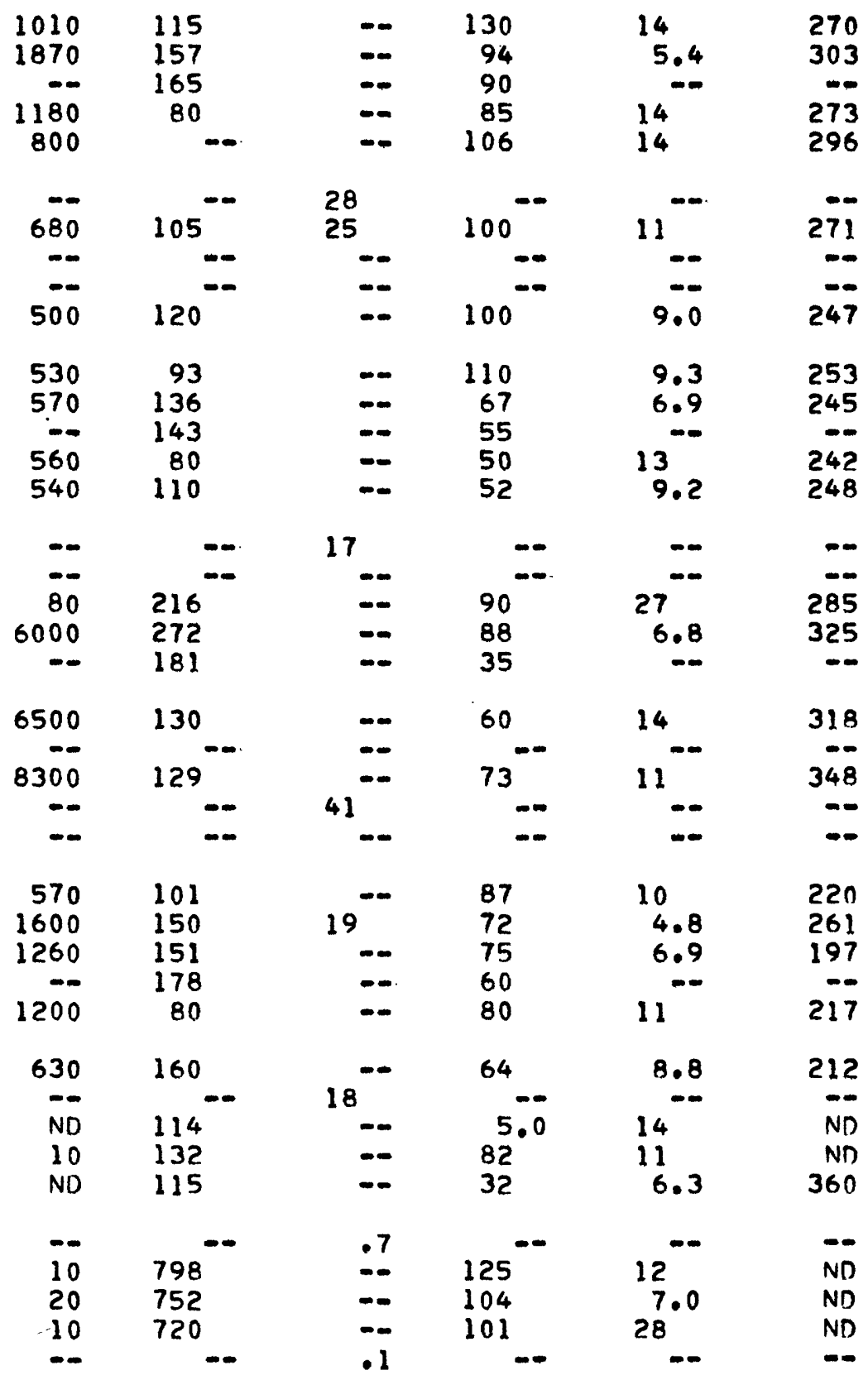




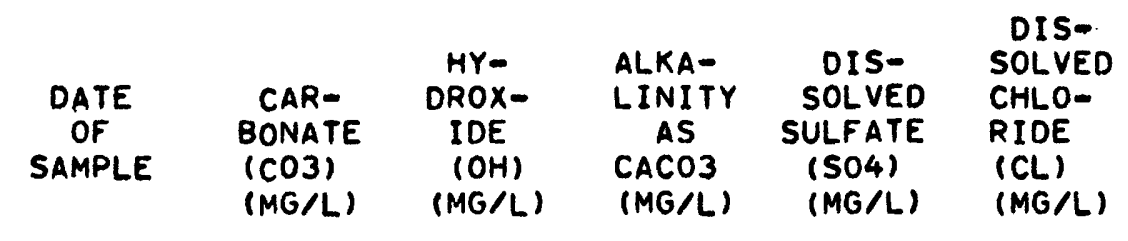

$74-11-25$

75-02-11

75-05-05

75-08-14

75-12-04

75-12-04

76-03-31

$74-10-11$

$74-10-11$

$74-11-25$

$74-11-25$

$75-02-10$

$75-05-05$

$75-08-14$

75-12-04

75-12-04

$76-04-16$

$74-11-25$

$75-02-11$

75-05-02

75-08-15

$75-08-15$

$75-12-09$

$75-12-09$

76-04-16

$74-11-25$

$75-02-10$

75-02-11

75-05-02

$75-08-15$

75-12-09

$75-12-09$

75-06-19

$75-08-20$

75-12-09

75-12-09

$75-06-19$

$75-08-20$

75-12-09

75-12-09

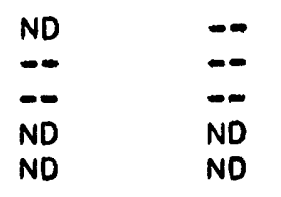

221

249

$-\infty$

224

243

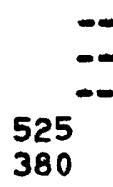

45

53

42

52

NO

--

ND

222
--
203

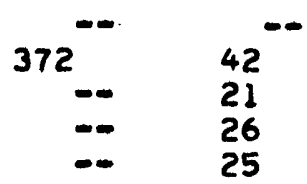

ND

$-\infty$

ND

ND

NO
$-\infty$
$-\infty$

-

$-$

$-\infty$

ND

ND

208

201

198

203

-0
$\therefore-$
-0

-0
234
267
-0

ND

ND

ND

$=$

$-$

ND

$-\infty$

$-$

ND

-

ND

$-\infty$

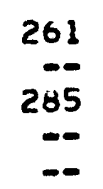

-

$-\infty$

$-9$

ND

180

214
162

178

ND

ND

$-2$

84

1420

330

66

NO

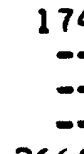

174

2660

$-\infty$

2860

2590

40

160

2220

380

$\begin{array}{rr}-- & 26 \\ -- & 27 \\ -- & 27 \\ 240 & 28 \\ & 26\end{array}$
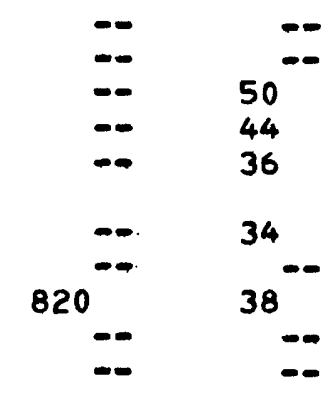

$\begin{array}{cc}370^{--} & 21 \\ -- & 16 \\ -- & 16 \\ 420 & 15 \\ & 17\end{array}$

440

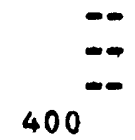

14

52

63

62

$-$

ND

$=\quad \quad N D$

$=\quad \quad N D$

9.0

20

25

--

--

$-\infty$

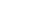


DIS-

$\begin{array}{lcccccc}\text { SOLVED } & \text { OIS- } & & \text { DIS } & \text { DIS- } & & \text { DIS } \\ \text { FLUO- } & \text { SOLVED } & \text { TOTAL } & \text { SOLVED } & \text { SOLVED } & \text { TOTALI } & \text { SOLVED } \\ \text { RIDE } & \text { NITRATE } & \text { NITRATE } & \text { NITRATE } & \text { NITRITE } & \text { NITRITE } & \text { NITRITE } \\ (F) & (N) & (N O 3) & (N O 3) & (N) & (N O 2) & (N O 2) \\ (M G / L) & (M G / L) & (M G / L) & (M G / L) & (M G / L) & (M G / L) & (M G / L)\end{array}$

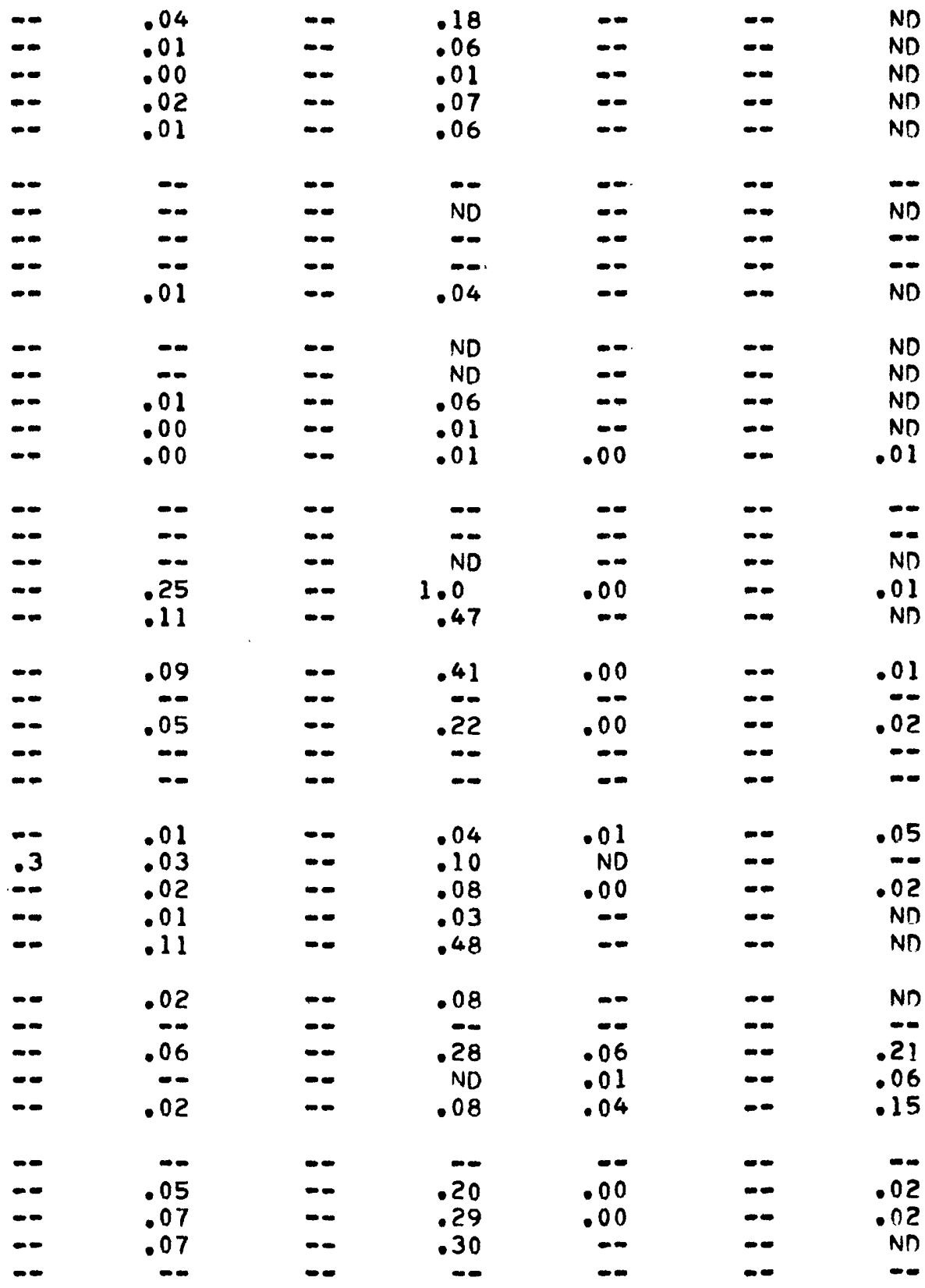




\begin{tabular}{|c|c|c|c|c|c|}
\hline $\begin{array}{l}\text { DATE } \\
\text { OF } \\
\text { SAMPLE }\end{array}$ & $\begin{array}{l}\text { OIS- } \\
\text { SOLVED } \\
\text { AMMONIA } \\
\text { NI TRO- } \\
\text { GEN } \\
\text { (N) } \\
\text { (MG/L) }\end{array}$ & $\begin{array}{l}\text { TOTAL } \\
\text { AMMONIA } \\
\text { (NH4) } \\
\text { (MG/L) }\end{array}$ & $\begin{array}{l}\text { DIS } \\
\text { SOLVED } \\
\text { AMMONIA } \\
\text { (NH4) } \\
\text { (MG/L) }\end{array}$ & $\begin{array}{l}\text { DIS- } \\
\text { SOLVED } \\
\text { ORGANIC } \\
\text { NITRO- } \\
\text { GEN } \\
\text { (N) } \\
\text { (MG/L) }\end{array}$ & $\begin{array}{l}\text { DIS- } \\
\text { SOLVED } \\
\text { KJEL. } \\
\text { NITRO- } \\
\text { GEN } \\
\text { (N) } \\
\text { (MG/L) }\end{array}$ \\
\hline $\begin{array}{l}74-11-25 \\
75-02-11 \\
75-05-05 \\
75-08-14 \\
75-12-04\end{array}$ & $\begin{array}{l}\text { ND } \\
\text { ND } \\
10 \\
\text { ND } \\
\text { ND }\end{array}$ & $\begin{array}{l}-- \\
-- \\
--\end{array}$ & -1 & $\begin{array}{l}-\infty \\
-0 \\
-0 \\
-\infty\end{array}$ & $\begin{array}{l}.80 \\
.70 \\
.10 \\
.30 \\
.30\end{array}$ \\
\hline $\begin{array}{l}75-12-04 \\
76-03-31 \\
74-10-11 \\
74-10-11 \\
74-11-25\end{array}$ & $\begin{array}{l}-10 \\
-10 \\
-\infty\end{array}$ & $\begin{array}{l}=- \\
-\infty \\
-\infty\end{array}$ & $\begin{array}{l}-13 \\
-- \\
--\end{array}$ & $\begin{array}{l}-0 \\
-\infty \\
-- \\
--\end{array}$ & $\begin{array}{r}.- \\
.40 \\
-- \\
.- \\
.30\end{array}$ \\
\hline $\begin{array}{l}74-11-25 \\
75=02-10 \\
75-05-05 \\
75-08-14 \\
75-12-04\end{array}$ & $\begin{array}{r}\text { ND } \\
.10 \\
\text { ND } \\
10 \\
\text { ND }\end{array}$ & $\begin{array}{l}-\infty \\
-\infty \\
-\infty\end{array}$ & $\begin{array}{r}.13 \\
.13 \\
0 . \\
-13\end{array}$ & $\begin{array}{c}2.4 \\
-\infty \\
=-\end{array}$ & $\begin{array}{r}1.3 \\
2.5 \\
.20 \\
.05 \\
.30\end{array}$ \\
\hline $\begin{array}{l}75-12-04 \\
76-04-16 \\
74-11-25 \\
75-02-11 \\
75-05-02\end{array}$ & $\begin{array}{r}-\infty \\
. \infty \\
\text { NO } \\
.80 \\
.50\end{array}$ & $\begin{array}{l}-- \\
-\infty \\
-\infty\end{array}$ & $\begin{array}{c}- \\
- \\
- \\
1.0 \\
.64\end{array}$ & $\begin{array}{c}-= \\
-- \\
-2 \\
3.2 \\
.30\end{array}$ & $\begin{array}{l}-. \\
4.5 \\
4.0 \\
.80\end{array}$ \\
\hline $\begin{array}{l}75-08-15 \\
75-08-15 \\
75-12-09 \\
75-12-09 \\
76-04-16\end{array}$ & $\begin{array}{r}.80 \\
.50 \\
.- \\
.-\end{array}$ & $\begin{array}{l}-\infty \\
-- \\
-\infty\end{array}$ & $\begin{array}{c}1.0 \\
.6 \\
.64 \\
-0 \\
\ldots\end{array}$ & $\begin{array}{r}.20 \\
.50 \\
.5 \\
-0\end{array}$ & $\begin{array}{c}1.0 \\
1.0 \\
-\infty \\
-\infty\end{array}$ \\
\hline $\begin{array}{l}74-11-25 \\
75-02-10 \\
75-02-11 \\
75-05-02 \\
75-08-15\end{array}$ & $\begin{array}{l}\text { ND } \\
.06 \\
\text { ND } \\
\text { ND } \\
\text { ND }\end{array}$ & $\begin{array}{l}-\infty \\
-\infty \\
-\infty \\
-\infty\end{array}$ & $\begin{array}{l}-\infty \\
.08 \\
-\infty \\
-\infty\end{array}$ & $\begin{array}{r}-\infty \\
.23 \\
-- \\
--\end{array}$ & $\begin{array}{r}\text { NO } \\
.29 \\
.80 \\
\text { NO } \\
.20\end{array}$ \\
\hline $\begin{array}{l}75-12-09 \\
75-12-09 \\
75-06-19 \\
75-08-20 \\
75-12-09\end{array}$ & $\begin{array}{l}\text { ND } \\
-- \\
.30 \\
.20 \\
\text { ND }\end{array}$ & $\begin{array}{l}-- \\
-\infty \\
-\infty \\
-\infty\end{array}$ & $\begin{array}{l}-- \\
-- \\
.39 \\
.26 \\
--\end{array}$ & $\begin{array}{c}-\infty \\
1.1 \\
-\infty\end{array}$ & $\begin{array}{c}.20 \\
. .4 \\
1.4 \\
.70\end{array}$ \\
\hline $\begin{array}{l}75-12-09 \\
75-06-19 \\
75-08-20 \\
75-12-09 \\
75-12-09\end{array}$ & $\begin{array}{r}-.- \\
2.9 \\
1.8 \\
.10 \\
--\end{array}$ & $\begin{array}{l}-- \\
-- \\
--\end{array}$ & $\begin{array}{l}-. \\
3.7 \\
2.3 \\
.13 \\
--\end{array}$ & $\begin{array}{c}-\infty \\
.50 \\
-\infty \\
1.8 \\
-\infty\end{array}$ & $\begin{array}{c}3.4 \\
1.9 \\
--\end{array}$ \\
\hline
\end{tabular}




$\begin{array}{lllllll}\text { DIS- } & \text { DIS- } & \text { DIS- } & \text { DIS- } & \text { DIS- } & & \\ \text { SOLVEO } & \text { SOLVED } & \text { SOLVED } & \text { SOLVED } & \text { SOLVED } & & \text { NON- } \\ \text { ORTHO. } & \text { ORTHO } & \text { SOLIDS } & \text { SOLIDS } & \text { SOLIDS } & & \text { CAR- } \\ \text { PHOS- } & \text { PHOS- } & \text { (RESI- } & \text { (RESI- } & \text { (SUM OF } & \text { HARD- } & \text { BONATE } \\ \text { PHORUS } & \text { PHATE } & \text { DUE AT } & \text { DUE AT } & \text { CONSTI- } & \text { NESS } & \text { HARD- } \\ (P) & (P O 4) & \left.180^{\circ} C\right) & \left.105^{\circ} C\right) & \text { TUENTS) } & \text { (CA MG) } & \text { NESS } \\ (M G / L) & (M G / L) & (M G / L) & (M G / L) & \text { (MG/L) } & \text { (MG/L) } & \text { (MG/L) }\end{array}$

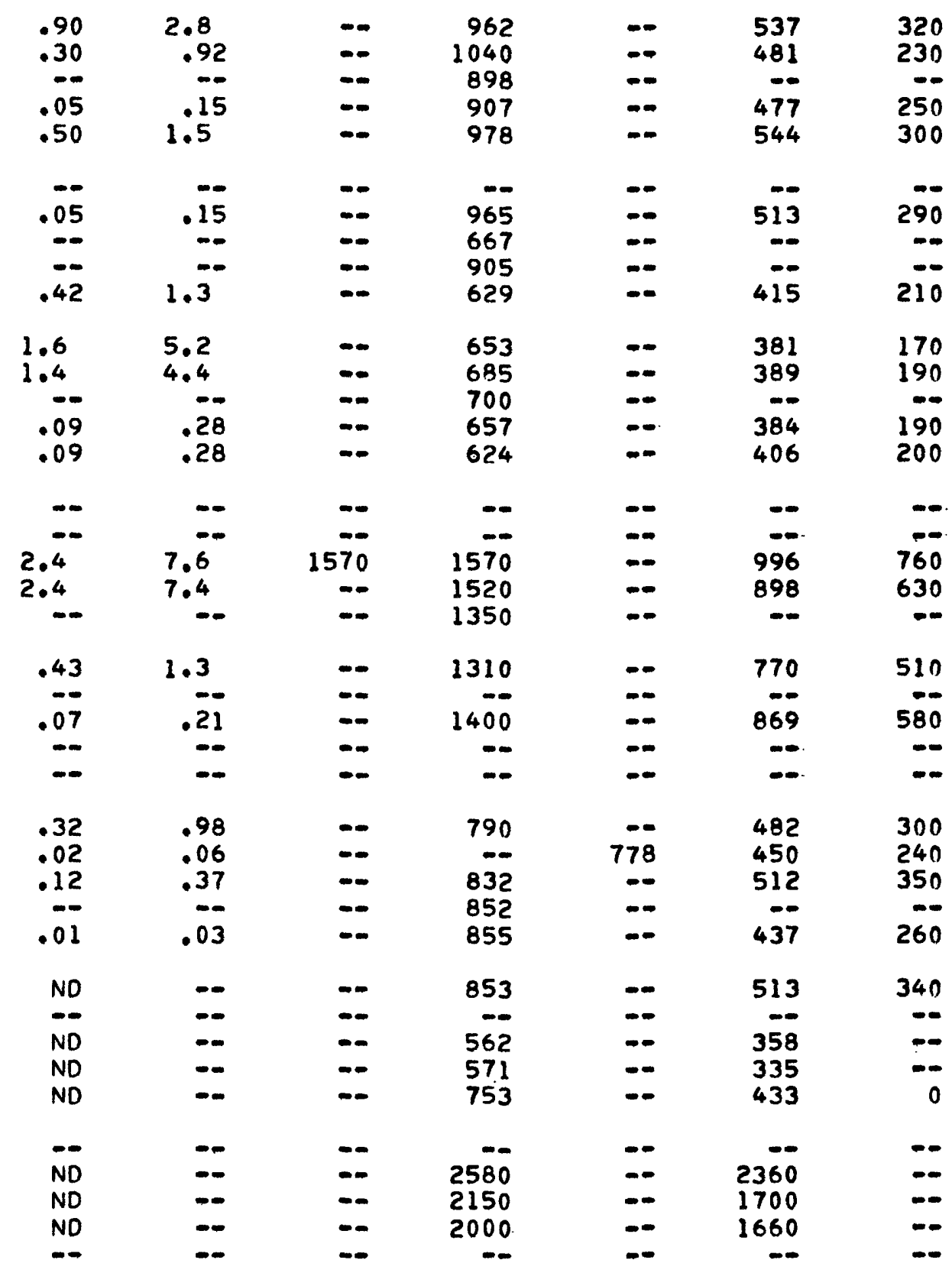


Table 5.--Chemical analyses of

\begin{tabular}{|c|c|c|c|c|c|c|}
\hline $\begin{array}{c}\text { DATE } \\
\text { OF } \\
\text { SAMPLE }\end{array}$ & $\begin{array}{l}\text { SPE- } \\
\text { CIFIC } \\
\text { CON- } \\
\text { DUCT- } \\
\text { ANCE } \\
\text { IMICRO- }\end{array}$ & $\mathrm{PH}$ & $\begin{array}{l}\text { TEMPER- } \\
\text { ATURE }\end{array}$ & $\begin{array}{l}\text { CARBON } \\
\text { OIOXIDE } \\
\text { (CO2) }\end{array}$ & $\begin{array}{l}\text { FECAL } \\
\text { COLI- } \\
\text { FORM } \\
\text { ICOL. } \\
\text { PER }\end{array}$ & $\begin{array}{l}\text { STREP- } \\
\text { TOCOCCI } \\
\text { ICOL- } \\
\text { ONIES } \\
\text { PER }\end{array}$ \\
\hline & MHOSI & (UNI TS) & & & $100 \mathrm{ML})$ & $100 \mathrm{ML})$ \\
\hline $\begin{array}{l}74-11-25 \\
75-02-11 \\
75-05=05 \\
75-08-14 \\
75-12-04\end{array}$ & $\begin{array}{l}1400 \\
1450 \\
1400 \\
1550 \\
1450\end{array}$ & $\begin{array}{l}7.5 \\
7.2 \\
7.4 \\
7.5 \\
7.6\end{array}$ & $\begin{array}{r}12.5 \\
6.0 \\
7.5 \\
14.0 \\
12.0\end{array}$ & $\begin{array}{l}14 \\
31 \\
14 \\
12\end{array}$ & $\begin{array}{r}-- \\
1 \\
-- \\
--\end{array}$ & $\begin{array}{r}-- \\
-- \\
--\end{array}$ \\
\hline $\begin{array}{l}75-12-04 \\
76-03-31 \\
74-10-11 \\
74-10-11 \\
74-11-25\end{array}$ & $\begin{array}{r}1450 \\
1225 \\
-- \\
=- \\
950\end{array}$ & $\begin{array}{r}7.6 \\
7.4 \\
.0 \\
. .0\end{array}$ & $\begin{array}{r}12.0 \\
7.5 \\
-- \\
--\end{array}$ & $17^{-\infty}$ & $\begin{array}{l}-\infty \\
-- \\
--\end{array}$ & $\begin{array}{l}-\infty \\
-\infty \\
-\infty \\
-\infty\end{array}$ \\
\hline $\begin{array}{l}74-11-25 \\
75-02-10 \\
75-05-05 \\
75-08-14 \\
75-12-04\end{array}$ & $\begin{array}{l}950 \\
1020 \\
1075 \\
1075 \\
1020\end{array}$ & $\begin{array}{l}7.1 \\
7.3 \\
7.1 \\
7.7 \\
7.4\end{array}$ & $\begin{array}{r}12.0 \\
9.0 \\
7.0 \\
12.0 \\
13.0\end{array}$ & $\begin{array}{l}32 \\
20 \\
7.7 \\
16\end{array}$ & $\begin{array}{r}-- \\
1 \\
-- \\
--\end{array}$ & $\begin{array}{r}-- \\
-- \\
-- \\
--\end{array}$ \\
\hline $\begin{array}{l}75-12-04 \\
76-04-16 \\
74-11-25 \\
75-02-11 \\
75-05-02\end{array}$ & $\begin{array}{r}1020 \\
20 \\
2050 \\
1900 \\
1590\end{array}$ & $\begin{array}{r}7.4 \\
-. \\
7.3 \\
6.9 \\
7.3\end{array}$ & $\begin{array}{r}13.0 \\
12.5 \\
12.5 \\
10.0\end{array}$ & $\begin{array}{l}-\infty \\
20 \\
65 \\
\ldots\end{array}$ & $\begin{array}{l}-- \\
-- \\
--\end{array}$ & $\begin{array}{r}-- \\
-- \\
--\end{array}$ \\
\hline $\begin{array}{l}75-08-15 \\
75-08-15 \\
75-12-09 \\
75-12-09 \\
76-04-16\end{array}$ & $\begin{array}{r}1950 \\
=- \\
2100 \\
2100 \\
--\end{array}$ & $\begin{array}{l}7.6 \\
-- \\
7.5 \\
7.5 \\
-.-\end{array}$ & $\begin{array}{r}13.5 \\
-.5 \\
12.0 \\
12.0 \\
.-\end{array}$ & $18^{13}-$ & $\begin{array}{l}= \\
-- \\
= \\
--\end{array}$ & $\begin{array}{l}-\infty \\
-\infty \\
-\infty\end{array}$ \\
\hline $\begin{array}{l}74-11-25 \\
75-02-10 \\
75-02-11 \\
75-05-02 \\
75-08-15\end{array}$ & $\begin{array}{r}1300 \\
1175 \\
1175 \\
900 \\
1325\end{array}$ & $\begin{array}{l}7.0 \\
7.1 \\
7.1 \\
7.6 \\
7.6\end{array}$ & $\begin{array}{r}11.0 \\
9.5 \\
9.5 \\
11.5 \\
11.5\end{array}$ & $\begin{array}{r}35 \\
33 \\
25 \\
8.7\end{array}$ & $\begin{array}{r}-- \\
-- \\
--\end{array}$ & $\begin{array}{c}-- \\
-- \\
--\end{array}$ \\
\hline $\begin{array}{l}75-12-09 \\
75-12-09 \\
75-06-19 \\
75-08-20 \\
75-12-09\end{array}$ & $\begin{array}{l}1350 \\
1350 \\
1650 \\
1025 \\
1150\end{array}$ & $\begin{array}{r}8.1 \\
8.1 \\
11.2 \\
10.8 \\
10.7\end{array}$ & $\begin{array}{l}11.0 \\
11.0 \\
10.0 \\
12.5 \\
12.0\end{array}$ & $\begin{array}{l}2.7 \\
-- \\
-. \\
.1\end{array}$ & $\begin{array}{l}-\infty \\
-- \\
-- \\
-\infty\end{array}$ & $\begin{array}{l}-\infty \\
-\infty \\
-\infty \\
-\infty\end{array}$ \\
\hline $\begin{array}{l}75-12-09 \\
75-06-19 \\
75-08-20 \\
75-12-09 \\
75-12-09\end{array}$ & $\begin{aligned} & 1150 \\
> & 8000 \\
> & 8000 \\
> & 8000 \\
> & 8000\end{aligned}$ & $\begin{array}{l}10.7 \\
11.8 \\
11.7 \\
12.4 \\
12.4\end{array}$ & $\begin{array}{l}12.0 \\
12.5 \\
13.0 \\
12.5 \\
12.5\end{array}$ & $\begin{array}{l}= \\
=- \\
-\infty \\
-\infty\end{array}$ & $\begin{array}{l}-\infty \\
-- \\
-- \\
--\end{array}$ & $\begin{array}{l}-- \\
\therefore \\
-\infty\end{array}$ \\
\hline
\end{tabular}


water from welzs--Continued

\begin{tabular}{|c|c|c|c|c|c|}
\hline $\begin{array}{c}\text { CYANIDE } \\
\text { (CN) } \\
\text { (MG/L) }\end{array}$ & $\begin{array}{l}\text { PHENOLS } \\
\text { (UG/L) }\end{array}$ & $\begin{array}{l}\text { TOTAL } \\
\text { ALDRIN } \\
\text { (UG/L) }\end{array}$ & $\begin{array}{l}\text { TOTAL } \\
\text { CHLOR- } \\
\text { DANE } \\
\text { (UG/L) }\end{array}$ & $\begin{array}{l}\text { TOTAL } \\
\text { DOD } \\
(U G / L)\end{array}$ & $\begin{array}{l}\text { TOTAL } \\
\text { DDE } \\
\text { (UG/L) }\end{array}$ \\
\hline & & & & $-\infty$ & $\infty$ \\
\hline$-\infty$ & $-\infty$ & - & $-\infty$ & $-\infty$ & $\infty$ \\
\hline$-\infty$ & $-\infty$ & $-\infty$ & -- & $m$ & $-\infty$ \\
\hline$-\infty$ & $-\infty$ & $-\infty$ & $-\infty$ & $\infty$ & - \\
\hline$-\infty$ & $-\infty$ & $-\infty$ & - & $-\infty$ & $-\infty$ \\
\hline$-\infty$ & $-\infty$ & $\infty$ & $-\infty$ & $-\infty$ & $-\infty$ \\
\hline$\infty$ & $-\infty$ & $-\infty$ & -- & $-\infty$ & $=-$ \\
\hline$-\infty$ & - & $-\infty$ & $-\infty$ & $-\infty$ & $\cdots$ \\
\hline$\rightarrow$ & $-\infty$ & $-\infty$ & -- & $\infty$ & - \\
\hline$\cdots$ & -- & $\cdots$ & - & $\infty$ & $\cdots$ \\
\hline$-\infty$ & - & $\ldots$ & $\infty$ & $\infty$ & - \\
\hline- & $-\infty$ & - & $\infty$ & $\infty$ & 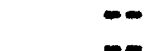 \\
\hline$-\infty$ & $-\infty$ & $\infty$ & $-\infty$ & - & - \\
\hline$-\infty$ & $-\infty$ & $-\infty$ & $-\infty$ & $\infty$ & - \\
\hline$\cdots$ & $-\infty$ & -- & $\cdots$ & $-\infty$ & $\cdots$ \\
\hline$-\infty$ & -- & $\ldots$ & $-\infty$ & -- & $-\infty$ \\
\hline$-\infty$ & - & $-\infty$ & $-\infty$ & $-\infty$ & $=$ \\
\hline$-\infty$ & $-\infty$ & - & $-\infty$ & $-\infty$ & $-\infty$ \\
\hline$-\infty$ & $-\infty$ & 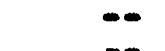 & $\because$ & $-\infty$ & $=$ \\
\hline- & $\infty$ & $\cdots$ & $\infty$ & $-\infty$ & - \\
\hline$\infty$ & $\infty$ & $\infty$ & -- & $\infty$ & $\ddot{-}$ \\
\hline- & - & ND & ND & ND & ND \\
\hline$-\infty$ & $-\infty$ & $\cdots$ & $\cdots$ & $\infty$ & - \\
\hline$-\infty$ & $=$ & $-\infty$ & $-\infty$ & 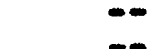 & $\because$ \\
\hline$-\infty$ & $-\infty$ & -- & $\cdots$ & $\cdots$ & - \\
\hline$-\infty$ & $\infty$ & - & $-\infty$ & $-\infty$ & - \\
\hline$-\infty$ & - & -- & -- & $-\infty$ & $=$ \\
\hline$\infty$ & $-\infty$ & $\infty$ & -- & $-\infty$ & - \\
\hline$-\infty$ & $-\infty$ & $\cdots$ & $=$ & $-\infty$ & $=$ \\
\hline 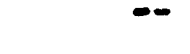 & $\infty$ & $\cdots$ & $-\infty$ & $-\infty$ & - \\
\hline$\infty$ & $-\infty$ & - & $-\infty$ & $-\infty$ & 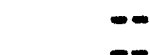 \\
\hline- & - & - & $-\infty$ & $\infty$ & - \\
\hline$-\infty$ & $\infty$ & $=$ & 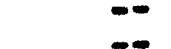 & $-\infty$ & $-\infty$ \\
\hline$\infty$ & $=$ & $=$ & $-\infty$ & $\infty$ & - \\
\hline$\cdots$ & - & $\cdots$ & - & 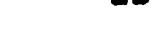 & 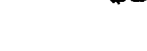 \\
\hline$=$ & -- & - & $\cdots$ & $-\infty$ & $-\infty$ \\
\hline$=$ & $=$ & 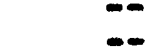 & 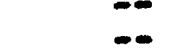 & $-\infty$ & - \\
\hline$\ldots$ & $=$ & $\ldots$ & $-\infty$ & $-\infty$ & $\cdots$ \\
\hline-- & $-\infty$ & $-\infty$ & 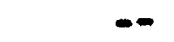 & $\ldots$ & \\
\hline
\end{tabular}


Table 5.--Chemical analyses of

\begin{tabular}{|c|c|c|c|c|c|c|}
\hline $\begin{array}{c}\text { DATE } \\
\text { OF } \\
\text { SAMPLE }\end{array}$ & $\begin{array}{l}\text { TOTAL } \\
\text { OOT } \\
\text { (UG/L) }\end{array}$ & $\begin{array}{l}\text { TOTAL } \\
\text { DI- } \\
\text { AZINON } \\
\text { (UG/L) }\end{array}$ & $\begin{array}{l}\text { TOTAL } \\
\text { DI- } \\
\text { ELDRIN } \\
\text { (UG/L) }\end{array}$ & $\begin{array}{l}\text { TOTAL } \\
\text { ENDRIN } \\
\text { (UG/L) }\end{array}$ & $\begin{array}{l}\text { TOTAL } \\
\text { HEPTA- } \\
\text { CHLOR } \\
\text { (UG/L) }\end{array}$ & $\begin{array}{l}\text { TOTAL } \\
\text { HEPTA- } \\
\text { CHLOR } \\
\text { EPOXIDE } \\
\text { (UG/L) }\end{array}$ \\
\hline
\end{tabular}

\begin{tabular}{|c|c|c|c|c|c|c|}
\hline $\begin{array}{l}74-11-25 \\
75-02-11 \\
75-05-05 \\
75-08-14 \\
75-12-04\end{array}$ & $\begin{array}{l}-\infty \\
-\infty \\
-\infty \\
--\end{array}$ & $\begin{array}{l}-\infty \\
-\infty \\
-\infty\end{array}$ & $=$ & $\begin{array}{l}=- \\
=- \\
=-\end{array}$ & $\begin{array}{l}-\infty \\
-\infty \\
-\infty \\
-\infty\end{array}$ & $\begin{array}{l}-\infty \\
-\infty \\
-\infty \\
-\infty\end{array}$ \\
\hline $\begin{array}{l}75-12-04 \\
76-03-31 \\
74-10-11 \\
74-10-11 \\
74-11-25\end{array}$ & $\begin{array}{l}-- \\
-- \\
-- \\
--\end{array}$ & $\begin{array}{l}= \\
-\infty \\
-\infty\end{array}$ & $\begin{array}{l}-\infty \\
-\infty \\
-\infty\end{array}$ & $\begin{array}{l}=- \\
=- \\
=-\end{array}$ & $\begin{array}{l}-\infty \\
-\infty \\
-\infty \\
-\infty\end{array}$ & $\begin{array}{l}-\infty \\
-\infty \\
-\infty \\
-\infty\end{array}$ \\
\hline $\begin{array}{l}74-11-25 \\
75-02-10 \\
75-05-05 \\
75-08-14 \\
75-12-04\end{array}$ & $\begin{array}{l}=- \\
=- \\
=- \\
--\end{array}$ & $\begin{array}{l}-- \\
-- \\
-\infty\end{array}$ & $\begin{array}{l}-\infty \\
-- \\
-\infty \\
-\infty\end{array}$ & $=$ & $\begin{array}{l}-\infty \\
-\infty \\
-\infty\end{array}$ & $\begin{array}{l}-\infty \\
-\infty \\
-\infty\end{array}$ \\
\hline $\begin{array}{l}75-12-04 \\
76-04-16 \\
74-11-25 \\
75-02-11 \\
75-05-02\end{array}$ & $\begin{array}{l}-- \\
-- \\
--\end{array}$ & $\begin{array}{l}-- \\
-- \\
-- \\
--\end{array}$ & $\begin{array}{l}-\infty \\
-\infty \\
-\infty \\
-\infty\end{array}$ & $\begin{array}{l}=- \\
-\infty \\
-\infty \\
-\infty\end{array}$ & $\begin{array}{l}-\infty \\
-\infty \\
-\infty \\
-\infty\end{array}$ & $\begin{array}{l}-\infty \\
-\infty \\
-\infty\end{array}$ \\
\hline $\begin{array}{l}75-08-15 \\
75-08-15 \\
75-12-09 \\
75-12-09 \\
76-04-16\end{array}$ & $\begin{array}{l}-\infty \\
\text { ND } \\
-- \\
--\end{array}$ & $\begin{array}{l}-- \\
-- \\
--\end{array}$ & $\begin{array}{l}-\infty \\
N D \\
-\infty \\
-\infty\end{array}$ & $\begin{array}{l}-- \\
-- \\
--\end{array}$ & $\begin{array}{l}-\infty \\
\text { NO } \\
-- \\
--\end{array}$ & $\begin{array}{l}-\infty \\
-\infty \\
-\infty \\
-\infty\end{array}$ \\
\hline $\begin{array}{l}74-11-25 \\
75-02-10 \\
75-02-11 \\
75-05-02 \\
75-08-15\end{array}$ & $\begin{array}{l}=- \\
-- \\
--\end{array}$ & $\begin{array}{l}=- \\
=- \\
=- \\
--\end{array}$ & $\begin{array}{l}= \\
= \\
= \\
-\infty\end{array}$ & $\begin{array}{l}-\infty \\
-- \\
--\end{array}$ & $\begin{array}{l}-\infty \\
-\infty \\
-\infty \\
-\infty\end{array}$ & $\begin{array}{l}-\infty \\
-\infty \\
-\infty\end{array}$ \\
\hline $\begin{array}{l}75-12-09 \\
75-12-09 \\
75-06-19 \\
75-08-20 \\
75-12-09\end{array}$ & $\begin{array}{l}=- \\
-- \\
-\infty\end{array}$ & $\begin{array}{l}=- \\
-- \\
-- \\
--\end{array}$ & $\begin{array}{l}-\infty \\
-\infty \\
-\infty \\
-\infty\end{array}$ & $\begin{array}{l}= \\
= \\
-\infty\end{array}$ & $\begin{array}{l}-- \\
-\infty \\
-\infty \\
-\infty\end{array}$ & $\because$ \\
\hline $\begin{array}{l}75-12-09 \\
75-06-19 \\
75-08-20 \\
75-12-09 \\
75-12-09\end{array}$ & $=-$ & $\begin{array}{l}-- \\
-- \\
--\end{array}$ & $\begin{array}{l}-\infty \\
-- \\
--\end{array}$ & $\begin{array}{l}= \\
-- \\
=\end{array}$ & $=-$ & - \\
\hline
\end{tabular}


water from wells--Continued

\begin{tabular}{|c|c|c|c|c|c|}
\hline $\begin{array}{l}\text { TOTAL } \\
\text { LINDANE } \\
\text { (UG/L! }\end{array}$ & $\begin{array}{l}\text { TOTAL } \\
\text { MALAC } \\
\text { THION } \\
\text { (UG/L) }\end{array}$ & $\begin{array}{l}\text { TOTAL } \\
\text { METHYL } \\
\text { PARA- } \\
\text { THION } \\
\text { (UG/L) }\end{array}$ & $\begin{array}{l}\text { TOTAL } \\
\text { PARA- } \\
\text { THION } \\
\text { (UG/L) }\end{array}$ & $\begin{array}{l}\text { TOTAL } \\
\text { PCB } \\
\text { (UG/L) }\end{array}$ & $\begin{array}{l}\text { TOTAL } \\
\text { TOX- } \\
\text { APHENE } \\
\text { (UG/L) }\end{array}$ \\
\hline-- & - & $-\infty$ & $-\infty$ & - & $-\infty$ \\
\hline- & $-\infty$ & $-\infty$ & - & $-\infty$ & $-\infty$ \\
\hline$-\infty$ & $\infty$ & -- & $-\infty$ & $-\infty$ & $-\infty$ \\
\hline$-\infty$ & $\infty$ & $-\infty$ & $-\infty$ & $-\infty$ & $-\infty$ \\
\hline-- & $\infty$ & $-\infty$ & - & - & $-\infty$ \\
\hline- & - & - & - & - & $\infty$ \\
\hline-- & - & - & $-\infty$ & $\cdots$ & \\
\hline$-\infty$ & $-\infty$ & - & - & $=$ & \\
\hline- & $-\infty$ & - & - & - & \\
\hline- & $-\infty$ & -- & - & $\cdots$ & \\
\hline$-\infty$ & 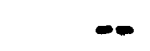 & - & - & - & 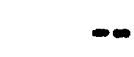 \\
\hline- & $-\infty$ & $\infty$ & $-\infty$ & - & - \\
\hline$-\infty$ & $-\infty$ & - & $-\infty$ & $-\infty$ & \\
\hline$-\infty$ & $-\infty$ & - & - & - & \\
\hline- & $\cdots$ & $\cdots$ & $-\infty$ & - & \\
\hline- & $\infty$ & - & $\infty$ & - & - \\
\hline-- & - & -- & $\cdots$ & $-\infty$ & \\
\hline-- & $-\infty$ & $-\infty$ & - & - & \\
\hline$\infty$ & $-\infty$ & $-\infty$ & $=-$ & - & \\
\hline$-\infty$ & $\infty$ & $\infty$ & $\cdots$ & $\cdots$ & \\
\hline$-\infty$ & $-\infty$ & $-\infty$ & - & - & \\
\hline ND & ND & ND & ND & .2 & ND \\
\hline$-\infty$ & - & $-\infty$ & $\cdots$ & - & \\
\hline- & $-\infty$ & $\infty$ & - & $-\infty$ & \\
\hline- & - & $-\infty$ & $\cdots$ & - & \\
\hline- & $-\infty$ & $-\infty$ & - & - & \\
\hline- & -- & - & $-\infty$ & - & \\
\hline- & $-\infty$ & $\infty$ & $-\infty$ & - & \\
\hline- & - & $-\infty$ & $\rightarrow$ & 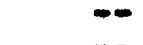 & \\
\hline$\cdots$ & $\cdots$ & $\cdots$ & $\infty$ & $=$ & \\
\hline$-\infty$ & $-\infty$ & - & $-\infty$ & $-\infty$ & \\
\hline$-\infty$ & $-\infty$ & $\infty$ & $\infty$ & $-\infty$ & \\
\hline- & $-\infty$ & - & - & $-\infty$ & \\
\hline$-\infty$ & $-\infty$ & - & $-\infty$ & $-\infty$ & \\
\hline$-\infty$ & $-\infty$ & $\cdots$ & $-\infty$ & $\cdots$ & \\
\hline$-\infty$ & -- & $-\infty$ & $-\infty$ & $\ldots$ & \\
\hline$-\infty$ & $\infty$ & $=$ & 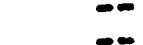 & $=$ & \\
\hline$-\infty$ & - & $\ldots$ & $=$ & $\ldots$ & \\
\hline$-\infty$ & $\cdots$ & - & $-\infty$ & $-\infty$ & \\
\hline
\end{tabular}


Table 5.--Chemical analyses of

\begin{tabular}{|c|c|c|c|c|}
\hline $\begin{array}{c}\text { DATE } \\
\text { OF } \\
\text { SAMPLE }\end{array}$ & $\begin{array}{l}\text { DIS- } \\
\text { SOLVED } \\
\text { CAD- } \\
\text { MIUM } \\
\text { (CD) } \\
(U G / L)\end{array}$ & $\begin{array}{l}\text { DIS- } \\
\text { SOLVED } \\
\text { CHRO- } \\
\text { MIUM } \\
\text { (CR) } \\
\text { (UG/L) }\end{array}$ & $\begin{array}{l}\text { DIS- } \\
\text { SOLVED } \\
\text { COPPER } \\
\text { (CU) } \\
\text { (UG/L) }\end{array}$ & $\begin{array}{l}\text { DIS- } \\
\text { SOLVED } \\
\text { LEAD } \\
\text { (PB) } \\
\text { (UG/L) }\end{array}$ \\
\hline $\begin{array}{l}74-11-25 \\
75-02-11 \\
75-05-05 \\
75-08-14 \\
75-12-04\end{array}$ & $\begin{array}{r}\text { ND } \\
2 \\
-- \\
\text { NO } \\
2\end{array}$ & $\begin{array}{l}30 \\
10 \\
=- \\
10 \\
\text { NO }\end{array}$ & $\begin{array}{l}\text { ND } \\
10 \\
-\infty \\
30 \\
10\end{array}$ & $\begin{array}{l}\text { ND } \\
30 \\
-- \\
\text { ND } \\
\text { ND }\end{array}$ \\
\hline $\begin{array}{l}75-12-04 \\
76-03-31 \\
74-10-11 \\
74-10-11 \\
74-11-25\end{array}$ & $\begin{array}{l}-- \\
-- \\
--\end{array}$ & $\begin{array}{l}-\infty \\
-- \\
--\end{array}$ & $\begin{array}{l}-\infty \\
10 \\
-\infty \\
\text { ND }\end{array}$ & $\begin{array}{l}-0 \\
100 \\
-\infty \\
20\end{array}$ \\
\hline $\begin{array}{l}74-11-25 \\
75-02-10 \\
75-05-05 \\
75-08-14 \\
75-12-04\end{array}$ & $\begin{array}{l}\text { ND } \\
\text { ND } \\
-- \\
\text { ND } \\
6\end{array}$ & $\begin{array}{l}20 \\
\text { ND } \\
-10 \\
10 \\
\text { ND }\end{array}$ & $\begin{array}{l}\text { ND } \\
\text { ND } \\
-- \\
20 \\
10\end{array}$ & $\begin{array}{l}10 \\
30 \\
-- \\
10 \\
30\end{array}$ \\
\hline $\begin{array}{l}75-12-04 \\
76-04-16 \\
74-11-25 \\
75-02-11 \\
75-05-02\end{array}$ & $\begin{array}{r}-- \\
-- \\
--\end{array}$ & $\begin{array}{l}-- \\
30 \\
20 \\
--\end{array}$ & $\begin{array}{l}-- \\
\text { ND } \\
10\end{array}$ & $\begin{array}{l}-- \\
10 \\
30 \\
--\end{array}$ \\
\hline $\begin{array}{l}75-08-15 \\
75-08-15 \\
75-12-09 \\
75-12-09 \\
76-04-16\end{array}$ & $\begin{array}{c}\text { ND } \\
-- \\
-- \\
--\end{array}$ & $\begin{array}{l}30 \\
-- \\
\text { ND } \\
--\end{array}$ & $\begin{array}{l}30 \\
-- \\
20 \\
--\end{array}$ & $\begin{array}{l}\text { ND } \\
=- \\
50 \\
=- \\
=-\end{array}$ \\
\hline $\begin{array}{l}74-11-25 \\
75-02-10 \\
75-02-11 \\
75-05-02 \\
75-08-15\end{array}$ & $\begin{array}{r}\text { ND } \\
-- \\
-2 \\
-\infty \\
\text { ND }\end{array}$ & $\begin{array}{l}40 \\
-\infty \\
\text { ND } \\
-\infty\end{array}$ & $\begin{array}{l}\text { ND } \\
-- \\
10 \\
30\end{array}$ & $\begin{array}{l}10 \\
10 \\
10\end{array}$ \\
\hline $\begin{array}{l}75-12-09 \\
75-12-09 \\
75-06-19 \\
75-08-20 \\
75-12-09\end{array}$ & $\begin{array}{l}\text { NO } \\
-- \\
\text { ND } \\
\text { ND } \\
3\end{array}$ & $\begin{array}{l}\text { ND } \\
-- \\
10 \\
\text { ND } \\
\text { ND }\end{array}$ & $\begin{array}{r}10 \\
-- \\
40 \\
390 \\
20\end{array}$ & $\begin{array}{l}30 \\
-- \\
50 \\
\text { NO } \\
20\end{array}$ \\
\hline $\begin{array}{l}75-12-09 \\
75-06-19 \\
75-08-20 \\
75-12-09 \\
75-12-09\end{array}$ & $\begin{array}{r}-- \\
\text { ND } \\
6 \\
---\end{array}$ & $\begin{array}{l}-- \\
30 \\
30 \\
10 \\
--\end{array}$ & $\begin{array}{l}-- \\
30 \\
40 \\
--\end{array}$ & $\begin{array}{r}-- \\
220 \\
160 \\
--\end{array}$ \\
\hline
\end{tabular}


water from wezzs--Continued

\begin{tabular}{|c|c|c|c|c|}
\hline $\begin{array}{c}\text { DIS- } \\
\text { SOLVED } \\
\text { MERCURY } \\
\text { (HG) } \\
(U G / L)\end{array}$ & $\begin{array}{l}\text { DIS- } \\
\text { SOLVED } \\
\text { NICKEL } \\
\text { (NI) } \\
(U G / L)\end{array}$ & $\begin{array}{l}\text { DIS- } \\
\text { SOLVED } \\
\text { ZINC } \\
(Z N) \\
(U G / L)\end{array}$ & $\begin{array}{l}\text { CODE } \\
\text { FOR } \\
\text { AGENCY } \\
\text { ANA- } \\
\text { LYZING } \\
\text { SAMPLE }\end{array}$ & $\begin{array}{c}\text { TOTAL } \\
\text { DEPTH } \\
\text { OF } \\
\text { WELL } \\
\text { (FT) }\end{array}$ \\
\hline $\begin{array}{l}-- \\
-- \\
-- \\
--\end{array}$ & $\begin{array}{l}\text { ND } \\
\text { ND } \\
-- \\
30 \\
\text { ND }\end{array}$ & $\begin{array}{r}100 \\
\text { ND } \\
-0 \\
20 \\
110\end{array}$ & $\begin{array}{l}9999 \\
9999 \\
9999 \\
9999 \\
9999\end{array}$ & $\begin{array}{l}16 \\
16 \\
16 \\
16 \\
16\end{array}$ \\
\hline $\begin{array}{l}-- \\
-- \\
-\infty\end{array}$ & $\begin{array}{l}-- \\
20 \\
-- \\
-\end{array}$ & $\begin{array}{l}-- \\
60 \\
-- \\
50\end{array}$ & $\begin{array}{r}-0 \\
9999 \\
9999 \\
9999 \\
9999\end{array}$ & $\begin{array}{l}16 \\
16 \\
11 \\
24 \\
17\end{array}$ \\
\hline $\begin{array}{l}-\infty \\
-\infty \\
-\infty \\
-\infty\end{array}$ & $\begin{array}{l}\text { ND } \\
\text { ND } \\
=- \\
30 \\
10\end{array}$ & $\begin{array}{l}10 \\
N D \\
-2 \\
10 \\
40\end{array}$ & $\begin{array}{l}9999 \\
9999 \\
9999 \\
9999 \\
9999\end{array}$ & $\begin{array}{l}17 \\
17 \\
17 \\
17 \\
17\end{array}$ \\
\hline $\begin{array}{l}-\infty \\
-\infty \\
-\infty \\
-\infty\end{array}$ & $\begin{array}{l}-- \\
20 \\
20 \\
--\end{array}$ & $\begin{array}{l}-\infty \\
-\infty \\
20 \\
20 \\
--\end{array}$ & $\begin{array}{r}-- \\
9999 \\
9999 \\
9999\end{array}$ & $\begin{array}{l}17 \\
17 \\
17 \\
17 \\
17\end{array}$ \\
\hline $\begin{array}{l}-\infty \\
-- \\
-- \\
--\end{array}$ & $\begin{array}{l}10 \\
-- \\
\text { ND } \\
-- \\
--\end{array}$ & $\begin{array}{l}70 \\
-0 \\
170 \\
-- \\
--\end{array}$ & $\begin{array}{r}9999 \\
9999 \\
=- \\
=-\end{array}$ & $\begin{array}{l}17 \\
17 \\
17 \\
17 \\
17\end{array}$ \\
\hline $\begin{array}{l}= \\
=- \\
=- \\
=-\end{array}$ & $\begin{array}{l}20 \\
20 \\
20 \\
-\infty\end{array}$ & $\begin{array}{l}50 \\
-- \\
20 \\
=- \\
\text { NO }\end{array}$ & $\begin{array}{r}9999 \\
9999 \\
9999 \\
9999\end{array}$ & $\begin{array}{l}33 \\
33 \\
33 \\
33 \\
33\end{array}$ \\
\hline $\begin{array}{l}-\infty \\
-- \\
-- \\
-\infty\end{array}$ & $\begin{array}{l}\text { ND } \\
-- \\
30 \\
60 \\
10\end{array}$ & $\begin{array}{l}50 \\
-0 \\
10 \\
50 \\
\text { ND }\end{array}$ & $\begin{array}{r}9999 \\
-0 \\
9999 \\
9999 \\
9999\end{array}$ & $\begin{array}{l}33 \\
33 \\
18 \\
18 \\
18\end{array}$ \\
\hline $\begin{array}{l}=- \\
=- \\
=-\end{array}$ & $\begin{array}{l}30 \\
40 \\
\text { ND } \\
--\end{array}$ & $\begin{array}{l}-5 \\
40 \\
80 \\
60 \\
--\end{array}$ & $\begin{array}{r}9999 \\
9999 \\
9999 \\
--\end{array}$ & $\begin{array}{l}18 \\
107 \\
107 \\
107 \\
107\end{array}$ \\
\hline
\end{tabular}


Table 5.--Chemical analyses of

LOCAL
IDENT-
I-
FIER

SC00506504CAB2

SC00506504CAC

SC00506504CDD

SC0050650408C

SC00506505BDA

SC00506506ABC

SC00506506BDDl

SC00506506BD02

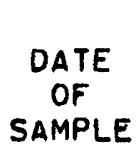

TOTAL
DEPTH
OF
WELL WELL

$76-04-01$
$74-10-11$
$74-11-25$
$75-02-10$
$75-05-05$

107

29

29

29

29

$75-08-19$

$75-12-08$

$75-12-08$

$76-04-01$

$74-10-11$

$74-11-25$

75-02-11

$75-03-20$

75-05-02

$75-08-15$

75-12-09

75-12-09

76-03-31

76-04-01

76-04-16

74-10-11

$74-11-27$

$74-11-26$

75-02-12

75-05-01

29

29

29

29

34

22

22

22

22

22

\section{2}

22

22

22

22

2101

2101

27

27

27

$75-08-18$

75-12-09

75-12-09

$76-03-31$

75-05-06

$75-06-19$

$75-08-20$

75-12-05

75-12-05

76-04-01

75-05-06

$75-06-19$

75-08-20

$75-12-05$

$75-12-05$

27

27

27

27

130

130

130

130

130

130

175

175

175

175

175
DIS-

SOLVEO

SILICA

(SIOZ)

(MG/L)
DIS-

SOLVED

IRON

(FE)

(UG/L)

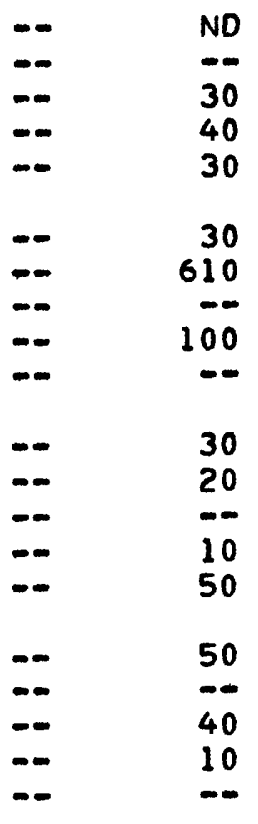

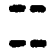

$-$

$-140$

- 100

-- 920

-- 490

-- 720

-- 550

-- $\quad 310$

$--\quad 310$
$--\quad 40$

-- 40

- $\quad 40$

-- 70

--

- 20

-- 20

$\begin{array}{ll}-- & 40\end{array}$

- 60

- 60 
water from wells--Continued

$\begin{array}{cccccc}\text { DIS- } & \text { DIS- } & \text { DIS- } & & \text { DIS- } \\ \text { SOLVED } & & \text { SOLVED } & \\ \text { SOLVED } & \text { SOLVED } & \text { MAG- } & \text { DIS- } & \text { PO- } & \\ \text { MAN- } & \text { CAL- } & \text { NE- } & \text { SOLVED } & \text { TAS- } & \text { BICAR- } \\ \text { GANESE } & \text { CIUM } & \text { SIUM } & \text { SODIUM } & \text { SIUM } & \text { BONATE } \\ \text { (MN) } & \text { (CA) } & \text { (MG) } & \text { (NA) } & \text { (K) } & \text { (HCO3) } \\ (U G / L) & (M G / L) & (M G / L) & (M G / L) & (M G / L) & (M G / L)\end{array}$

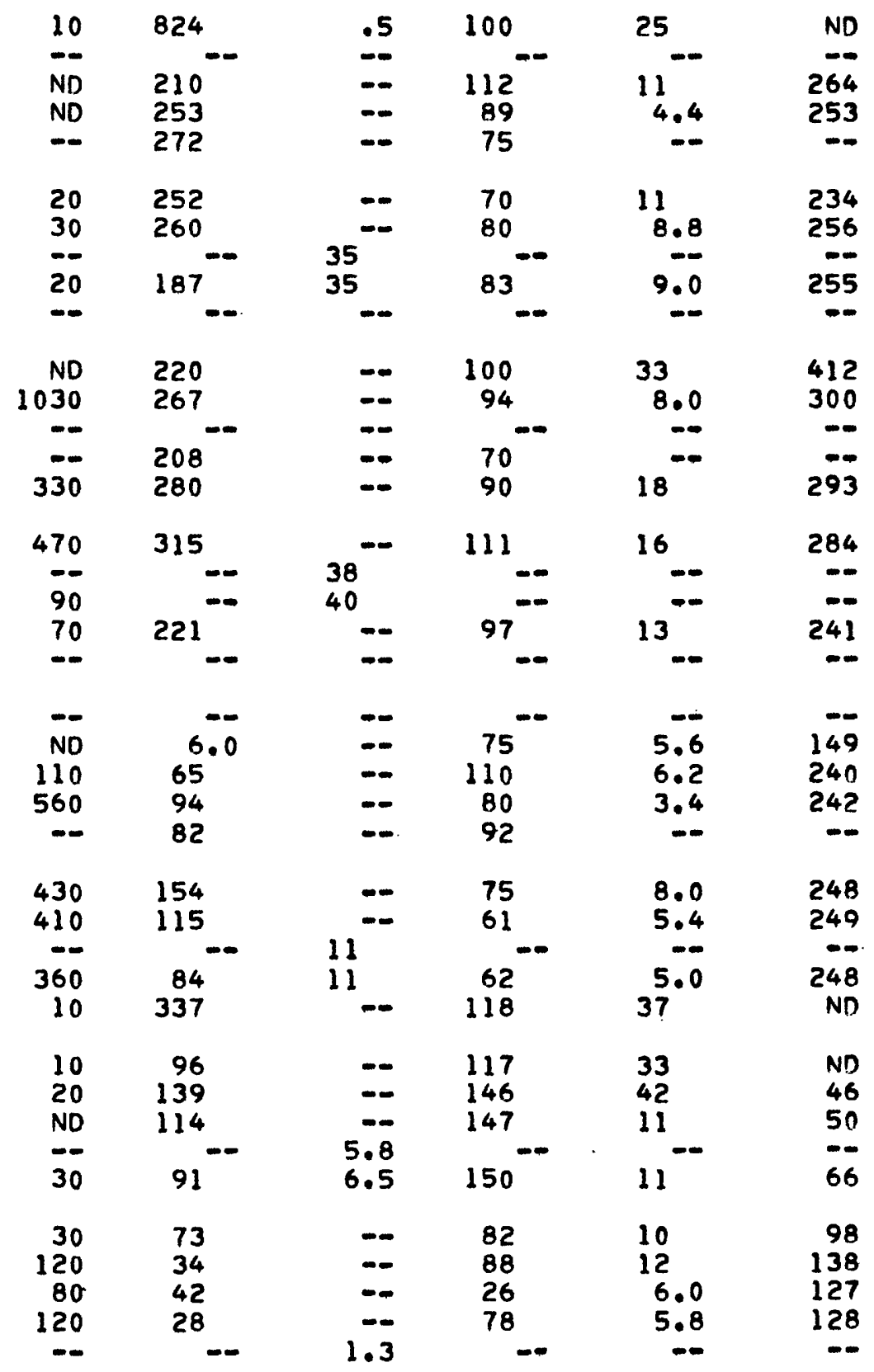




\begin{tabular}{|c|c|c|c|c|c|}
\hline $\begin{array}{c}\text { DATE } \\
\text { OF } \\
\text { SAMPLE }\end{array}$ & $\begin{array}{l}\text { CAR- } \\
\text { BONATE } \\
\text { (CO3) } \\
\text { (MG/L) }\end{array}$ & $\begin{array}{l}H Y- \\
O R O X- \\
I D E \\
(O H) \\
(M G / L)\end{array}$ & $\begin{array}{l}\text { ALKA- } \\
\text { LINITY } \\
\text { AS } \\
\text { CACO3 } \\
\text { (MG/L) }\end{array}$ & $\begin{array}{l}\text { DIS- } \\
\text { SOLVED } \\
\text { SULFATE } \\
\text { (SO4) } \\
\text { (MG/L) }\end{array}$ & $\begin{array}{l}\text { DIS- } \\
\text { SOLVED } \\
\text { CHLO- } \\
\text { RIDE } \\
\text { (CL) } \\
\text { (MG/L) }\end{array}$ \\
\hline $\begin{array}{l}76-04-01 \\
74-10-11 \\
74-11-25 \\
75-02-10 \\
75-05-05\end{array}$ & $\begin{array}{l}40 \\
-- \\
\text { ND } \\
-- \\
--\end{array}$ & $\begin{array}{r}2110 \\
=- \\
=- \\
=-\end{array}$ & $\begin{array}{r}-- \\
217 \\
208 \\
--\end{array}$ & $\begin{array}{l}\text { ND } \\
-- \\
-- \\
-\end{array}$ & $\begin{array}{l}31 \\
106 \\
119 \\
123 \\
125\end{array}$ \\
\hline $\begin{array}{l}75-08-19 \\
75-12-08 \\
75-12-08 \\
76-04-01 \\
74-10-11\end{array}$ & $\begin{array}{l}\text { ND } \\
\text { ND } \\
-- \\
\text { ND } \\
--\end{array}$ & $\begin{array}{l}\text { ND } \\
\text { ND } \\
-- \\
\text { ND } \\
--\end{array}$ & $\begin{array}{r}192 \\
210 \\
=- \\
209 \\
=-\end{array}$ & $\begin{array}{l}545 \\
660 \\
628\end{array}$ & $\begin{array}{l}121 \\
140 \\
140 \\
212\end{array}$ \\
\hline $\begin{array}{l}74-11-25 \\
75-02-11 \\
75-03-20 \\
75-05-02 \\
75-08-15\end{array}$ & $\begin{array}{l}\text { ND } \\
-- \\
-- \\
\text { ND }\end{array}$ & 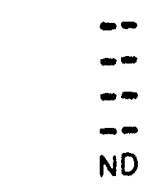 & $\begin{array}{r}338 \\
246 \\
-0 \\
240\end{array}$ & $375^{-\infty}$ & $\begin{array}{r}89 \\
119 \\
162 \\
239\end{array}$ \\
\hline $\begin{array}{l}75-12-09 \\
75-12-09 \\
76-03-31 \\
76-04-01 \\
76-04-16\end{array}$ & $\begin{array}{l}\text { ND } \\
-- \\
-- \\
--\end{array}$ & $\begin{array}{l}\text { ND } \\
-- \\
-- \\
\text { NO } \\
--\end{array}$ & $\begin{array}{r}233 \\
-0 \\
198 \\
--\end{array}$ & $300^{-\infty}$ & 200 \\
\hline $\begin{array}{l}74-10-11 \\
74-11-27 \\
74-11-26 \\
75-02-12 \\
75-05-01\end{array}$ & $\begin{array}{l}-- \\
\text { ND } \\
\text { ND } \\
--\end{array}$ & $\begin{array}{l}= \\
=- \\
=- \\
=-\end{array}$ & $\begin{array}{l}-- \\
122 \\
197 \\
198 \\
--\end{array}$ & $\begin{array}{l}=- \\
=- \\
= \\
=-\end{array}$ & $\begin{array}{l}4 \cdot 8 \\
4 \cdot 3 \\
11 \\
7 \cdot 0 \\
12\end{array}$ \\
\hline $\begin{array}{l}75-08-18 \\
75-12-09 \\
75-12-09 \\
76-03-31 \\
75-05-06\end{array}$ & $\begin{array}{l}\text { NO } \\
\text { ND } \\
-- \\
\text { ND } \\
24\end{array}$ & $\begin{array}{l}\text { ND } \\
\text { ND } \\
-- \\
\text { ND } \\
876\end{array}$ & $\begin{array}{r}203 \\
204 \\
-- \\
203 \\
--\end{array}$ & $\begin{array}{l}205 \\
180^{-0} \\
143 \\
315\end{array}$ & $\begin{array}{r}24 \\
18 \\
21 \\
103\end{array}$ \\
\hline $\begin{array}{l}75-06-19 \\
75-08-20 \\
75-12-05 \\
75-12-05 \\
76-04-01\end{array}$ & $\begin{array}{l}64 \\
\text { ND } \\
\text { ND } \\
-- \\
\text { ND }\end{array}$ & $\begin{array}{r}149 \\
\text { ND } \\
\text { ND } \\
-- \\
\text { ND }\end{array}$ & $\begin{array}{l}-2 \\
38 \\
41 \\
-\infty \\
54\end{array}$ & $\begin{array}{r}430 \\
90 \\
480 \\
466^{--}\end{array}$ & $\begin{array}{l}130 \\
122 \\
126 \\
118\end{array}$ \\
\hline $\begin{array}{l}75-05-06 \\
75-06-19 \\
75-08-20 \\
75-12-05 \\
75-12-05\end{array}$ & $\begin{array}{l}\text { ND } \\
\text { ND } \\
\text { ND } \\
\text { ND } \\
--\end{array}$ & $\begin{array}{l}-- \\
\text { ND } \\
\text { ND } \\
\text { ND } \\
--\end{array}$ & $\begin{array}{r}80 \\
113 \\
104 \\
105 \\
--\end{array}$ & $\begin{array}{r}170 \\
20 \\
100 \\
52\end{array}$ & $\begin{array}{l}58 \\
54 \\
56 \\
54\end{array}$ \\
\hline
\end{tabular}


DIS-

SOLVED

FLUO-

DIS-
SOLVED

DIS
TOTAL DISNITRATE NITRITE (N03) (NO3)

(N)

(N)

(MG/L)

(MG/L)

(MG/L)

DIS.

TOTAL SOLVED

NITRITE NITRITE

(NO2) (NO2)

(MG/L) (MG/L)

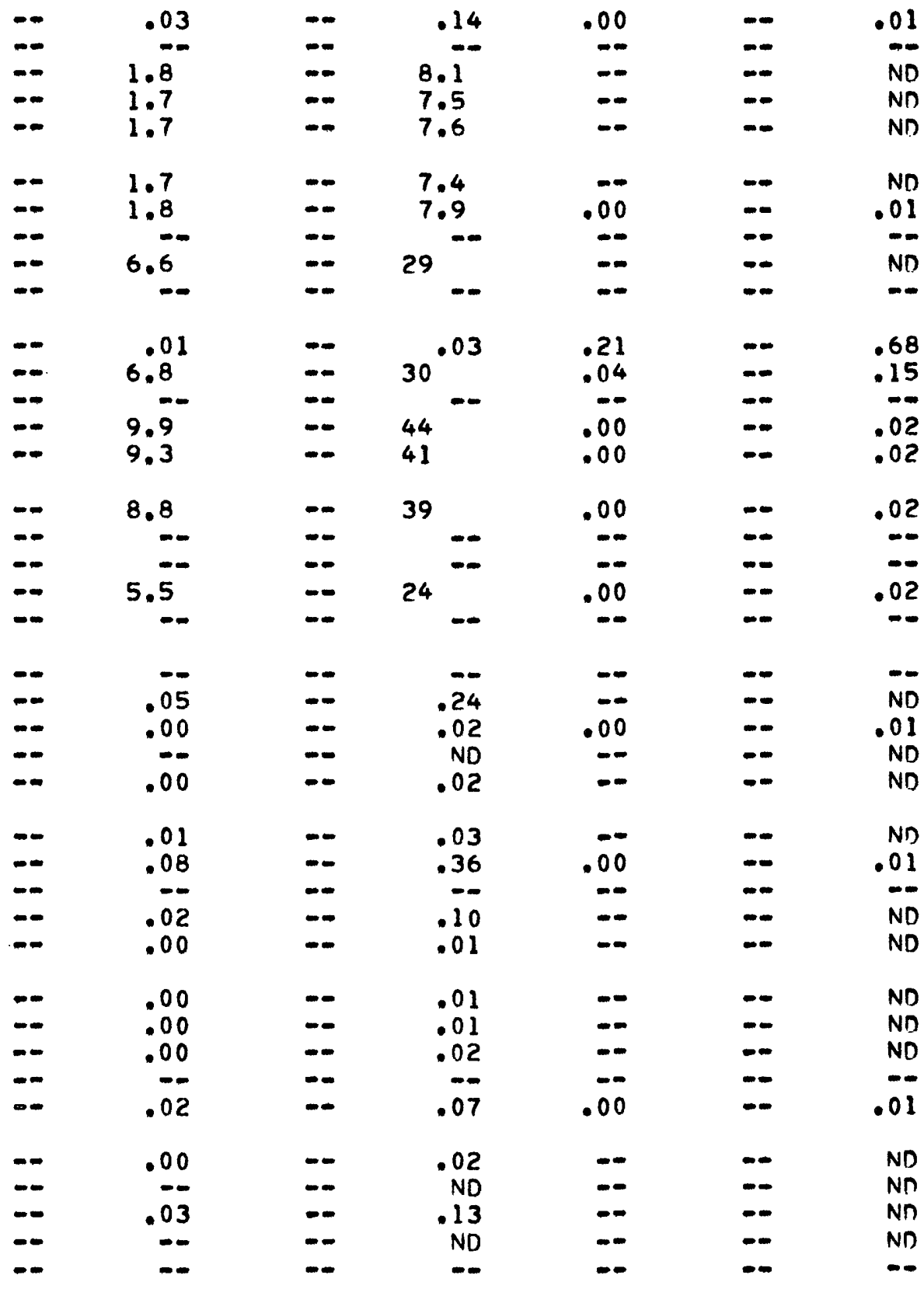


Table 5.--Chemical analyses of

\begin{tabular}{|c|c|c|c|c|c|}
\hline $\begin{array}{c}\text { DATE } \\
\text { OF } \\
\text { SAMPLE }\end{array}$ & $\begin{array}{l}\text { DIS- } \\
\text { SOLVED } \\
\text { AMMONIA } \\
\text { NITRO- } \\
\text { GEN } \\
\text { (N) } \\
\text { (MG/L) }\end{array}$ & $\begin{array}{l}\text { TOTAL } \\
\text { AMMONIA } \\
\text { (NH4) } \\
\text { (MG/L) }\end{array}$ & $\begin{array}{c}\text { DIS } \\
\text { SOLVED } \\
\text { AMMONIA } \\
(N H 4) \\
(M G / L)\end{array}$ & $\begin{array}{l}\text { DIS- } \\
\text { SOLVED } \\
\text { ORGANIC } \\
\text { NITRO- } \\
\text { GEN } \\
\text { (N) } \\
\text { (MG/L) }\end{array}$ & $\begin{array}{l}\text { DIS- } \\
\text { SOLVED } \\
\text { KJEL. } \\
\text { NITRO- } \\
\text { GEN } \\
\text { (N) } \\
(M G / L)\end{array}$ \\
\hline $\begin{array}{l}76-04-01 \\
74-10-11 \\
74-11-25 \\
75-02-10 \\
75-05-05\end{array}$ & $\begin{array}{r}1.4 \\
- \\
\text { ND } \\
\text { ND } \\
\text { ND }\end{array}$ & $\begin{array}{l}=- \\
=- \\
= \\
=-\end{array}$ & $\begin{array}{c}1.8 \\
=- \\
=- \\
=-\end{array}$ & $\begin{array}{c}2.8 \\
=- \\
=- \\
=\end{array}$ & $\begin{array}{r}4.2 \\
- \\
\text { ND } \\
50 \\
\text { ND }\end{array}$ \\
\hline $\begin{array}{l}75-08-19 \\
75-12-08 \\
75-12-08 \\
76-04-01 \\
74-10-11\end{array}$ & $\begin{array}{l}\text { ND } \\
\text { ND } \\
-- \\
\text { ND } \\
--\end{array}$ & $\begin{array}{l}-\infty \\
-\infty \\
-\infty\end{array}$ & $\begin{array}{l}-\infty \\
-\infty \\
-\infty \\
-\infty\end{array}$ & $\begin{array}{l}-\infty \\
-\infty \\
-\infty \\
-\infty\end{array}$ & $\begin{array}{r}.50 \\
.10 \\
.- \\
.40 \\
.-\end{array}$ \\
\hline $\begin{array}{l}74-11-25 \\
75-02-11 \\
75-03-20 \\
75-05-02 \\
75-08-15\end{array}$ & $\begin{array}{r}\text { ND } \\
.70 \\
.0 \\
.40 \\
.90\end{array}$ & $\begin{array}{l}= \\
=- \\
=- \\
-\infty\end{array}$ & $\begin{array}{r}.-50 \\
.90 \\
.52 \\
1.52\end{array}$ & $\begin{array}{c}1.6 \\
.0 \\
.10 \\
.70\end{array}$ & $\begin{array}{r}3.8 \\
2.3 \\
-.50 \\
1.56\end{array}$ \\
\hline $\begin{array}{l}75-12-09 \\
75-12-09 \\
76-03-31 \\
76-04-01 \\
76-04-16\end{array}$ & $\begin{array}{l}\text { ND } \\
-- \\
-- \\
\text { ND } \\
--\end{array}$ & $\begin{array}{l}-\infty \\
-\infty \\
-- \\
-\infty\end{array}$ & $\begin{array}{l}-\infty \\
-\infty \\
-- \\
--\end{array}$ & $\begin{array}{l}= \\
-\infty \\
=- \\
-\infty\end{array}$ & $\begin{array}{c}.60 \\
-. \\
.60 \\
-0\end{array}$ \\
\hline $\begin{array}{l}74-10-11 \\
74-11-27 \\
74-11-26 \\
75-02-12 \\
75-05-01\end{array}$ & $\begin{array}{l}-- \\
\text { ND } \\
\text { ND } \\
\text { ND } \\
\text { ND }\end{array}$ & $\begin{array}{l}=- \\
-- \\
=- \\
--\end{array}$ & $\begin{array}{l}-- \\
-\infty \\
-- \\
--\end{array}$ & $\begin{array}{l}-\infty \\
-\infty \\
-\infty \\
-\infty\end{array}$ & $\begin{array}{r}-- \\
\text { ND } \\
\text { ND } \\
.40 \\
.10\end{array}$ \\
\hline $\begin{array}{l}75-08-18 \\
75-12-09 \\
75-12-09 \\
76-03-31 \\
75-05-06\end{array}$ & $\begin{array}{r}\text { ND } \\
.10 \\
-.0 \\
\text { ND } \\
.10\end{array}$ & $\begin{array}{l}-\infty \\
-- \\
-- \\
--\end{array}$ & $\begin{array}{r}.13 \\
.13 \\
.13\end{array}$ & $\begin{array}{c}.70 \\
- \\
-0 \\
-0\end{array}$ & $\begin{array}{r}.30 \\
.80 \\
.50 \\
.50 \\
1.1\end{array}$ \\
\hline $\begin{array}{l}75-06-19 \\
75-08-20 \\
75-12-05 \\
75-12-05 \\
76-04-01\end{array}$ & $\begin{array}{r}.30 \\
.20 \\
.30 \\
-- \\
\text { ND }\end{array}$ & $\begin{array}{l}-- \\
-- \\
-- \\
--\end{array}$ & $\begin{array}{l}.39 \\
.26 \\
.39 \\
-- \\
--\end{array}$ & $\begin{array}{l}= \\
-30 \\
=- \\
=-\end{array}$ & $\begin{array}{r}.20 \\
.60 \\
.70\end{array}$ \\
\hline $\begin{array}{l}75-05-06 \\
75-06-19 \\
75-08-20 \\
75-12-05 \\
75-12-05\end{array}$ & $\begin{array}{r}\text { NO } \\
.10 \\
\text { ND } \\
.10 \\
--\end{array}$ & $\begin{array}{l}-- \\
-- \\
--\end{array}$ & 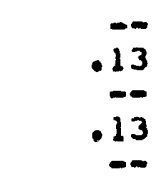 & $\begin{array}{l}.00 \\
.00 \\
.20\end{array}$ & $\begin{array}{l}.20 \\
.10 \\
.70 \\
.30 \\
-.\end{array}$ \\
\hline
\end{tabular}




\begin{tabular}{|c|c|c|c|c|c|c|}
\hline $\begin{array}{l}\text { OIS- } \\
\text { SOLVED } \\
\text { ORTHO. } \\
\text { PHOS- } \\
\text { PHORUS } \\
\text { (P) } \\
\text { (MG/L) }\end{array}$ & $\begin{array}{l}\text { DIS- } \\
\text { SOLVEO } \\
\text { ORTHO } \\
\text { PHOS- } \\
\text { PHATE } \\
\text { (PO4) } \\
\text { (MG/L) }\end{array}$ & $\begin{array}{l}\text { DIS- } \\
\text { SOLVED } \\
\text { SOLIDS } \\
\text { (RESI- } \\
\text { DUE AT } \\
180^{\circ} \mathrm{C} \text { ) } \\
(\mathrm{MG} / \mathrm{L})\end{array}$ & $\begin{array}{l}\text { DIS- } \\
\text { SOLVED } \\
\text { SOLIDS } \\
\text { (RESI- } \\
\text { DUE AT } \\
\left.105^{\circ} \mathrm{C}\right) \\
\text { (MG/L) }\end{array}$ & $\begin{array}{l}\text { DIS- } \\
\text { SOLVED } \\
\text { SOLIDS } \\
\text { (SUM OF } \\
\text { CONSTI- } \\
\text { TUENTS) } \\
\text { (MG/L) }\end{array}$ & $\begin{array}{l}\text { HARD- } \\
\text { NESS } \\
\text { (CA,MG) } \\
\text { (MG/L) }\end{array}$ & $\begin{array}{l}\text { NON- } \\
\text { CAR- } \\
\text { BONATE } \\
\text { HARD- } \\
\text { NESS } \\
\text { (MG/L) }\end{array}$ \\
\hline ND & $-\infty$ & - & 1980 & - & 2380 & - \\
\hline$\infty$ & $-\infty$ & - & 1180 & - & $=$ & - \\
\hline $\begin{array}{r}14 \\
\text { NO }\end{array}$ & .43 & - & $\begin{array}{l}1370 \\
1270\end{array}$ & $\Rightarrow$ & $\begin{array}{l}835 \\
853\end{array}$ & $\begin{array}{l}620 \\
650\end{array}$ \\
\hline$-\infty$ & $-\infty$ & - & 1360 & $-\infty$ & $\ldots$ & - \\
\hline $\begin{array}{r}\text { ND } \\
.01\end{array}$ & .03 & $=$ & $\begin{array}{l}1310 \\
1520\end{array}$ & $=$ & $\begin{array}{l}804 \\
922\end{array}$ & $\begin{array}{l}610 \\
710\end{array}$ \\
\hline - & $-\infty$ & - & $=$ & - & $-\infty$ & $\ldots$ \\
\hline ND & $-\infty$ & $\infty$ & 1450 & $-\infty$ & 917 & 710 \\
\hline$\infty$ & - & $\infty$ & 2620 & $-\infty$ & $-\infty$ & $-\infty$ \\
\hline $\begin{array}{l}1.1 \\
1.0\end{array}$ & $\begin{array}{l}3.5 \\
3.3\end{array}$ & $-\infty$ & $\begin{array}{l}1500 \\
1520\end{array}$ & $=$ & $\begin{array}{l}914 \\
834\end{array}$ & $\begin{array}{l}580 \\
590\end{array}$ \\
\hline- & - & - & - & -- & - & $-\infty$ \\
\hline .41 & $1.3^{--}$ & $=$ & $\begin{array}{l}1490 \\
1840\end{array}$ & $=$ & $10 \overline{20}$ & 780 \\
\hline .40 & 1.2 & - & 1640 & $\therefore$ & 914 & $68 n$ \\
\hline- & $-\infty$ & - & $-\infty$ & $\rightarrow$ & $-\infty$ & $-\infty$ \\
\hline$-\infty$ & $-\infty$ & - & $-\infty$ & - & - & - \\
\hline .34 & 1.0 & $-\infty$ & 1530 & - & 867 & 670 \\
\hline$\infty$ & $-\infty$ & $-\infty$ & $-\infty$ & - & - & $-\infty$ \\
\hline- & $\ldots$ & -- & 254 & - & $-\infty$ & $=$ \\
\hline $\begin{array}{r}.02 \\
.12\end{array}$ & $\begin{array}{l}.06 \\
.37\end{array}$ & 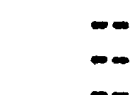 & $\begin{array}{l}215 \\
497\end{array}$ & $=$ & $\begin{array}{r}32 \\
277 \\
256\end{array}$ & $\begin{array}{r}0 \\
80 \\
57\end{array}$ \\
\hline ND & $=$ & $=$ & $\begin{array}{l}684 \\
526\end{array}$ & $=$ & 256 & $\begin{array}{l}57 \\
-\end{array}$ \\
\hline & & & & & & \\
\hline .02 & .06 & $-\infty$ & 619 & - & 329 & 130 \\
\hline NO & $-\infty$ & $-\infty$ & 552 & $-\infty$ & 299 & 95 \\
\hline$-\infty$ & $-\infty$ & - & $-\infty$ & - & $-\infty$ & $-\infty$ \\
\hline ND & - & $-\infty$ & 510 & $\infty$ & 319 & 120 \\
\hline ND & - & $-\infty$ & 1790 & - & 908 & $-\infty$ \\
\hline $\begin{array}{l}\text { ND } \\
.01 \\
.01\end{array}$ & $\begin{array}{l}.03 \\
.03\end{array}$ & $=$ & $\begin{array}{r}1090 \\
964 \\
951\end{array}$ & $=$ & $\begin{array}{l}446 \\
341 \\
342\end{array}$ & $\begin{array}{l}300 \\
300\end{array}$ \\
\hline- & - & $-\infty$ & $\infty$ & - & $-\infty$ & $-\infty$ \\
\hline .01 & .03 & - & 980 & $-\infty$ & 319 & 270 \\
\hline $\begin{array}{l}\text { ND } \\
.04 \\
.01 \\
\text { ND }\end{array}$ & $\begin{array}{l}.12 \\
.12 \\
.03 \\
-\infty\end{array}$ & $=$ & $\begin{array}{l}467 \\
308 \\
312 \\
282\end{array}$ & $\begin{array}{l}-\infty \\
-\infty \\
-\infty\end{array}$ & $\begin{array}{r}144 \\
83 \\
89 \\
98\end{array}$ & $\begin{array}{r}64 \\
0 \\
0 \\
0\end{array}$ \\
\hline- & $-\infty$ & - & 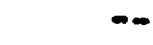 & & - & - \\
\hline
\end{tabular}




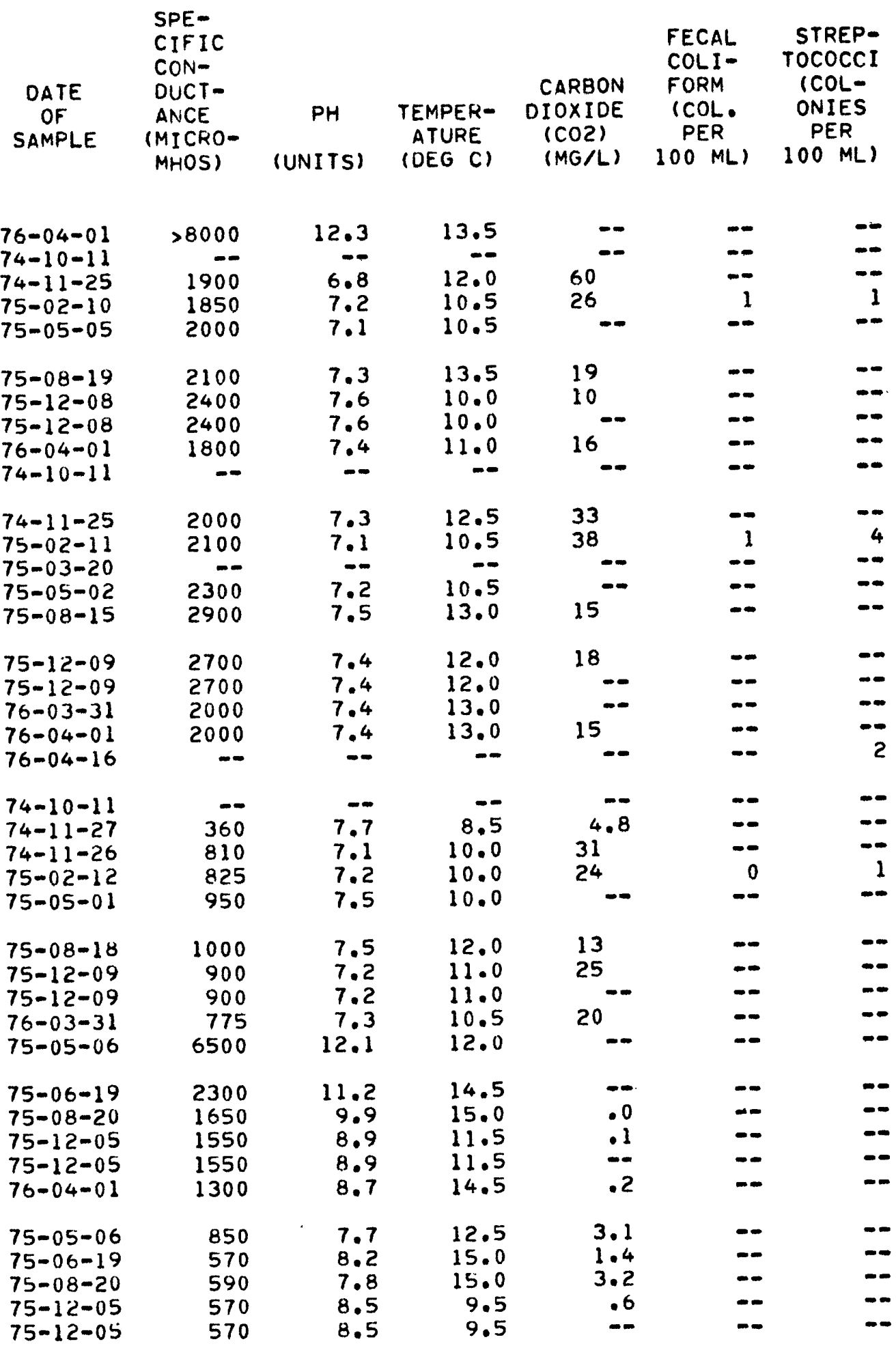


water from welzs--Continued

\begin{tabular}{|c|c|c|c|c|c|}
\hline $\begin{array}{c}\text { CYANIDE } \\
\text { (CN) } \\
(M G / L)\end{array}$ & $\begin{array}{l}\text { PHENOLS } \\
\text { (UG/L) }\end{array}$ & $\begin{array}{l}\text { TOTAL } \\
\text { ALDRIN } \\
\text { (UG/L) }\end{array}$ & $\begin{array}{l}\text { TOTAL } \\
\text { CHLOR- } \\
\text { OANE } \\
\text { (UG/L) }\end{array}$ & $\begin{array}{l}\text { TOTAL } \\
\text { DOD } \\
\text { (UG/L) }\end{array}$ & $\begin{array}{l}\text { TOTAL } \\
\text { DDE } \\
\text { (UG/L) }\end{array}$ \\
\hline$-\infty$ & -- & $-\infty$ & -- & $-\infty$ & -- \\
\hline -. & -- & - & -- & $-\infty$ & $\infty$ \\
\hline-- & $-\infty$ & $-\infty$ & -- & $-\infty$ & $-\infty$ \\
\hline 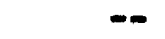 & -- & -- & $-\infty$ & $\cdots$ & -- \\
\hline-- & -- & -- & $-\infty$ & $-\infty$ & -- \\
\hline$-\infty$ & -- & -- & $\infty$ & $-\infty$ & - \\
\hline$\rightarrow$ & -- & $-\infty$ & - & $-\infty$ & 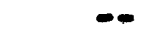 \\
\hline- & $-\infty$ & $-\infty$ & -0 & $-\infty$ & -- \\
\hline 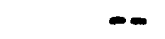 & $\rightarrow$ & -- & $\infty$ & $-\infty$ & $=-$ \\
\hline-- & $-\infty$ & -- & -- & $-\infty$ & - \\
\hline$-\infty$ & $-\infty$ & 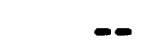 & -- & $-\infty$ & $-\infty$ \\
\hline- & -- & - & - & $-\infty$ & $\infty$ \\
\hline$-\infty$ & ND & -- & $-\infty$ & $-\infty$ & $\infty$ \\
\hline-- & -- & $-\infty$ & -- & -- & $\cdots$ \\
\hline$-\infty$ & -- & ND & NO & No & ND \\
\hline- & - & $\ldots$ & -- & $-\infty$ & -- \\
\hline-- & $\ldots$ & -- & $\cdots$ & $-\infty$ & $-\infty$ \\
\hline$\cdots$ & 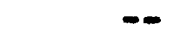 & 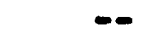 & -- & $-\infty$ & $\cdots$ \\
\hline$-\infty$ & -- & -- & -- & -- & $-\infty$ \\
\hline$-\infty$ & -- & -- & -- & $-\infty$ & -- \\
\hline$-\infty$ & $-\infty$ & $\ldots$ & -- & - & -- \\
\hline$-\infty$ & -- & 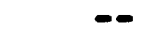 & - & -- & -- \\
\hline$-\infty$ & $-\infty$ & -- & - & $-\infty$ & $\cdots$ \\
\hline ND & -- & $-\infty$ & -- & $-\infty$ & -- \\
\hline$-\infty$ & $\cdots$ & $=$ & $\cdots$ & -- & -- \\
\hline- & -- & -- & $-\infty$ & $-\infty$ & -- \\
\hline-- & -- & $=-$ & - & $-\infty$ & $-\infty$ \\
\hline$\cdots$ & -- & - & -- & $-\infty$ & $-\infty$ \\
\hline$-\infty$ & $=$ & -- & $-\infty$ & - & $-\infty$ \\
\hline$-\infty$ & 15 & $-\infty$ & $\cdots$ & $-\infty$ & - \\
\hline$\ldots$ & -- & $\infty$ & -- & - & $-\infty$ \\
\hline$-\infty$ & $-\infty$ & -- & -- & -- & - \\
\hline$-\infty$ & $-\infty$ & $-\infty$ & $-\infty$ & - & $-\infty$ \\
\hline- & -- & $\infty$ & $-\infty$ & $-\infty$ & - \\
\hline-- & $-\infty$ & $\cdots$ & $-\infty$ & $-\infty$ & $-\infty$ \\
\hline-- & ND & $\cdots$ & $-\infty$ & $-\infty$ & $-\infty$ \\
\hline- & -- & -- & $-\infty$ & - & $=$ \\
\hline$\because$ & $=$ & 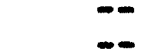 & $=-$ & $=$ & $=$ \\
\hline$=$ & 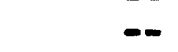 & - & - & $=$ & \\
\hline
\end{tabular}


Table 5.--Chemical analyses of

\begin{tabular}{|c|c|c|c|c|c|c|}
\hline $\begin{array}{c}\text { DATE } \\
\text { OF } \\
\text { SAMPLE }\end{array}$ & $\begin{array}{l}\text { TOTAL } \\
\text { DOT } \\
\text { (UG/L) }\end{array}$ & $\begin{array}{l}\text { TOTAL } \\
\text { DI- } \\
\text { AZINON } \\
\text { (UG/L) }\end{array}$ & $\begin{array}{l}\text { TOTAL } \\
\text { DI- } \\
\text { ELDRIN } \\
\text { (UG/L) }\end{array}$ & $\begin{array}{l}\text { TOTAL } \\
\text { ENDRIN } \\
\text { (UG/L) }\end{array}$ & $\begin{array}{l}\text { TOTAL } \\
\text { HEPTA- } \\
\text { CHLOR } \\
\text { (UG/L) }\end{array}$ & $\begin{array}{l}\text { HEPTA- } \\
\text { CHLOR } \\
\text { EPOXIDE } \\
\text { (UG/L) }\end{array}$ \\
\hline
\end{tabular}

\begin{tabular}{|c|c|c|c|c|c|c|}
\hline $\begin{array}{l}76-04-01 \\
74-10-11 \\
74-11-25 \\
75-02-10 \\
75-05-05\end{array}$ & $\begin{array}{l}-- \\
-\infty \\
-\infty\end{array}$ & $\begin{array}{l}= \\
=- \\
=- \\
-\infty\end{array}$ & $\begin{array}{l}=- \\
=- \\
=- \\
-\infty\end{array}$ & $\begin{array}{l}= \\
=- \\
=- \\
=0\end{array}$ & $\begin{array}{l}= \\
=- \\
=- \\
=\end{array}$ & $\begin{array}{l}-\infty \\
-\infty \\
-\infty \\
-\infty\end{array}$ \\
\hline $\begin{array}{l}75-08-19 \\
75-12-08 \\
75-12-08 \\
76-04-01 \\
74-10-11\end{array}$ & $\begin{array}{l}-- \\
-- \\
--\end{array}$ & $\begin{array}{l}= \\
=- \\
=- \\
=- \\
=-\end{array}$ & $\begin{array}{l}-\infty \\
-\infty \\
-\infty \\
-\infty\end{array}$ & $\begin{array}{l}-\infty \\
-\infty \\
-\infty \\
-\infty\end{array}$ & $\begin{array}{l}= \\
-\infty \\
= \\
-\infty\end{array}$ & $\begin{array}{l}= \\
=- \\
=- \\
-\infty\end{array}$ \\
\hline $\begin{array}{l}74-11-25 \\
75-02-11 \\
75-03-20 \\
75-05-02 \\
75-08-15\end{array}$ & $\begin{array}{l}-- \\
-- \\
-\infty \\
\text { NO }\end{array}$ & $\begin{array}{l}-- \\
-\infty \\
=- \\
\text { ND }\end{array}$ & $\begin{array}{l}-\infty \\
-\infty \\
-\infty \\
-\infty \\
\text { ND }\end{array}$ & $\begin{array}{l}- \\
-- \\
-\infty \\
\text { ND }\end{array}$ & $\begin{array}{l}= \\
-\infty \\
=- \\
=\end{array}$ & $=$ \\
\hline $\begin{array}{l}75-12-09 \\
75-12-09 \\
76-03-31 \\
76-04-01 \\
76-04-16\end{array}$ & $\begin{array}{l}=- \\
=- \\
=-\end{array}$ & $\begin{array}{l}- \\
-\infty \\
-\infty \\
-\infty\end{array}$ & $\begin{array}{l}- \\
- \\
-\infty \\
-\infty\end{array}$ & $\begin{array}{l}-\infty \\
= \\
= \\
=\end{array}$ & $\begin{array}{l}-\infty \\
-\infty \\
-\infty \\
-\infty\end{array}$ & $\begin{array}{l}-\infty \\
-\infty \\
-\infty \\
-\infty\end{array}$ \\
\hline $\begin{array}{l}74-10-11 \\
74-11-27 \\
74-11-26 \\
75-02-12 \\
75-05-01\end{array}$ & $=$ & $\begin{array}{l}-\infty \\
=- \\
-\infty \\
-\infty\end{array}$ & $\begin{array}{l}= \\
=- \\
=- \\
=-\end{array}$ & $\begin{array}{l}-\infty \\
-\infty \\
-\infty\end{array}$ & $\begin{array}{l}= \\
=- \\
= \\
=\end{array}$ & $\begin{array}{l}- \\
- \\
= \\
- \\
-\end{array}$ \\
\hline $\begin{array}{l}75-08-18 \\
75-12-09 \\
75-12-09 \\
76-03-31 \\
75-05-06\end{array}$ & $\begin{array}{l}-- \\
-- \\
--\end{array}$ & $\begin{array}{l}= \\
=- \\
=- \\
=- \\
=-\end{array}$ & $\begin{array}{l}-\infty \\
-\infty \\
-\infty \\
-\infty \\
-\infty\end{array}$ & $\begin{array}{l}-\infty \\
-\infty \\
-\infty \\
-\infty\end{array}$ & $\begin{array}{l}-\infty \\
-\infty \\
-\infty \\
-\infty\end{array}$ & $\begin{array}{l}-\infty \\
-\infty \\
-\infty\end{array}$ \\
\hline $\begin{array}{l}75-06-19 \\
75-08-20 \\
75-12-05 \\
75-12-05 \\
76-04-01\end{array}$ & $=$ & $\begin{array}{l}= \\
-\infty \\
-\infty \\
-\infty\end{array}$ & $\begin{array}{l}= \\
=- \\
=- \\
=-\end{array}$ & $\begin{array}{l}- \\
-\infty \\
- \\
-\end{array}$ & $\begin{array}{l}= \\
-- \\
=- \\
=- \\
-\infty\end{array}$ & $\begin{array}{l}= \\
-\infty \\
= \\
-\infty\end{array}$ \\
\hline $\begin{array}{l}75-05-06 \\
75-06-19 \\
75-08-20 \\
75-12-05 \\
75-12-05\end{array}$ & $=$ & $\begin{array}{l}-\infty \\
-\infty \\
-\infty \\
-\infty\end{array}$ & $\begin{array}{l}= \\
-\infty \\
-\infty \\
-\infty\end{array}$ & $\begin{array}{l}- \\
- \\
-\infty \\
-\infty\end{array}$ & $\begin{array}{l}= \\
-\infty \\
=- \\
-\infty\end{array}$ & $\begin{array}{l}= \\
-\infty \\
-\infty \\
-\infty\end{array}$ \\
\hline
\end{tabular}


water from wells--Continued

\begin{tabular}{llllll}
\multicolumn{1}{c}{ TOTAL } & & & \\
TOTAL & TOTAL & METHYL & TOTAL & & TOTAL \\
MINDAN & PARA- & PARA- & TOTAL & TOX- \\
LINDANE & THION & THION & THION & PCB & APHENE \\
$(U G / L)$ & $(U G / L)$ & $(U G / L)$ & $(U G / L)$ & $(U G / L)$ & $(U G / L)$
\end{tabular}

\begin{tabular}{|c|c|c|c|c|c|}
\hline$-\infty$ & $-\infty$ & - & $-\infty$ & $-\infty$ & $-\infty$ \\
\hline$-\infty$ & $\infty$ & - & $\infty$ & $\infty$ & - \\
\hline$-\infty$ & $-\infty$ & $-\infty$ & $\cdots$ & $-\infty$ & - \\
\hline$-\infty$ & $\infty$ & $-\infty$ & $-\infty$ & $-\infty$ & - \\
\hline$-\infty$ & $-\infty$ & $-\infty$ & $-\infty$ & - & - \\
\hline$-\infty$ & - & - & $\infty$ & $-\infty$ & \\
\hline$-\infty$ & $m$ & - & $-\infty$ & $\infty$ & \\
\hline$\infty$ & $-\infty$ & $-\infty$ & $-\infty$ & 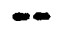 & \\
\hline$-\infty$ & $\infty$ & $\infty$ & $-\infty$ & $-\infty$ & \\
\hline$-\infty$ & $\infty$ & $-\infty$ & - & $\infty$ & - \\
\hline$-\infty$ & - & $\cdots$ & $-\infty$ & 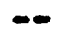 & \\
\hline$-\infty$ & 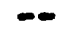 & $-\infty$ & $-\infty$ & $-\infty$ & \\
\hline$-\infty$ & $-\infty$ & $\cdots$ & $-\infty$ & $-\infty$ & \\
\hline$\infty$ & $\infty$ & $-\infty$ & -- & $-\infty$ & \\
\hline ND & ND & ND & ND & .2 & NO \\
\hline$-\infty$ & $\infty$ & $-\infty$ & $-\infty$ & $-\infty$ & \\
\hline$-\infty$ & $-\infty$ & $-\infty$ & $-\infty$ & $-\infty$ & \\
\hline$-\infty$ & $\infty$ & $\infty$ & $\cdots$ & $\infty$ & \\
\hline$-\infty$ & $\infty$ & $-\infty$ & $-\infty$ & $\infty$ & \\
\hline$\infty$ & $\infty$ & $\infty$ & $-\infty$ & $-\infty$ & \\
\hline$\infty$ & $-\infty$ & $-\infty$ & 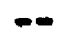 & $\cdots$ & \\
\hline-- & $\infty$ & - & 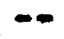 & $\cdots$ & \\
\hline-- & $\cdots$ & $\cdots$ & $\cdots$ & $-\infty$ & \\
\hline$\infty$ & $-\infty$ & 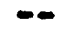 & $\cdots$ & - & \\
\hline$-\infty$ & $-\infty$ & - & $\infty$ & $\infty$ & \\
\hline$-\infty$ & $-\infty$ & - & $-\infty$ & $-\infty$ & \\
\hline$\cdots$ & $\infty$ & - & $-\infty$ & - & \\
\hline$-\infty$ & $\infty$ & $-\infty$ & - & $\cdots$ & \\
\hline$-\infty$ & $-\infty$ & - & - & - & \\
\hline$\cdots$ & $-\infty$ & $-\infty$ & $-\infty$ & $-\infty$ & \\
\hline$-\infty$ & $\infty$ & $-\infty$ & $-\infty$ & $-\infty$ & 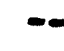 \\
\hline$\infty$ & $-\infty$ & - & $\cdots$ & $-\infty$ & \\
\hline$-\infty$ & $m$ & $-\infty$ & $-\infty$ & $-\infty$ & \\
\hline 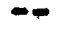 & $-\infty$ & $\cdots$ & $-\infty$ & $\infty$ & \\
\hline$-\infty$ & $-\infty$ & - & - & $\infty$ & \\
\hline$\infty$ & - & $\infty$ & $-\infty$ & $-\infty$ & \\
\hline$-\infty$ & $\infty$ & - & $-\infty$ & $-\infty$ & \\
\hline$-\infty$ & $=$ & $=$ & $=$ & $=$ & \\
\hline 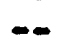 & 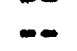 & $\infty$ & $=$ & $\ldots$ & \\
\hline
\end{tabular}


Table 5,--Chemical analyses of

\begin{tabular}{|c|c|c|c|c|}
\hline $\begin{array}{l}\text { DATE } \\
\text { OF } \\
\text { SAMPLE }\end{array}$ & $\begin{array}{l}\text { DIS- } \\
\text { SOLVEO } \\
\text { CAD- } \\
\text { MIUM } \\
\text { (CD) } \\
(U G / L)\end{array}$ & $\begin{array}{l}\text { DIS- } \\
\text { SOLVED } \\
\text { CHRO- } \\
\text { MIUM } \\
\text { (CR) } \\
\text { (UG/L) }\end{array}$ & $\begin{array}{l}\text { OIS- } \\
\text { SOLVED } \\
\text { COPPER } \\
\text { (CU) } \\
\text { (UG/L) }\end{array}$ & $\begin{array}{l}\text { DIS- } \\
\text { SOLVEO } \\
\text { LEAD } \\
\text { (PB) } \\
(U G / L)\end{array}$ \\
\hline $\begin{array}{l}76-04-01 \\
74-10-11 \\
74-11-25 \\
75-02-10 \\
75-05-05\end{array}$ & $\begin{array}{r}5 \\
-- \\
1 \\
--\end{array}$ & $\begin{array}{l}\text { NO } \\
-- \\
30 \\
\text { NO } \\
--\end{array}$ & $\begin{array}{l}60 \\
-0 \\
\text { ND } \\
10 \\
-\infty\end{array}$ & $\begin{array}{r}240 \\
-10 \\
30 \\
-\end{array}$ \\
\hline $\begin{array}{l}75-08-19 \\
75-12-08 \\
75-12-08 \\
76-04-01 \\
74-10-11\end{array}$ & $\begin{array}{r}\text { ND } \\
5 \\
-- \\
\text { ND } \\
--\end{array}$ & $\begin{array}{l}10 \\
20 \\
-- \\
20 \\
--\end{array}$ & $\begin{array}{l}20 \\
10 \\
-- \\
10 \\
--\end{array}$ & $\begin{array}{l}30 \\
10 \\
-- \\
70 \\
--\end{array}$ \\
\hline $\begin{array}{l}74-11-25 \\
75-02-11 \\
75-03-20 \\
75-05-02 \\
75-08-15\end{array}$ & $\begin{array}{l}\text { ND } \\
\text { ND } \\
-- \\
-- \\
\text { ND }\end{array}$ & $\begin{array}{l}60 \\
\text { ND } \\
-- \\
-- \\
30\end{array}$ & $\begin{array}{l}\text { ND } \\
10 \\
-- \\
100\end{array}$ & $\begin{array}{l}\text { ND } \\
30 \\
-- \\
-- \\
\text { ND }\end{array}$ \\
\hline $\begin{array}{l}75-12-09 \\
75-12-09 \\
76-03-31 \\
76-04-01 \\
76-04-16\end{array}$ & $\begin{array}{r}3 \\
-- \\
-- \\
--\end{array}$ & $\begin{array}{l}\text { ND } \\
-- \\
10 \\
--\end{array}$ & $\begin{array}{l}20 \\
-- \\
30 \\
--\end{array}$ & $\begin{array}{l}30 \\
-- \\
120 \\
--\end{array}$ \\
\hline $\begin{array}{l}74-10-11 \\
74-11-27 \\
74-11-26 \\
75-02-12 \\
75-05-01\end{array}$ & $\begin{array}{c}-- \\
1 \\
\text { ND } \\
--\end{array}$ & $\begin{array}{l}-- \\
\text { ND } \\
\text { ND } \\
10 \\
--\end{array}$ & $\begin{array}{l}-- \\
40 \\
\text { ND } \\
\text { ND } \\
--\end{array}$ & $\begin{array}{l}-- \\
\text { ND } \\
70 \\
\text { ND } \\
--\end{array}$ \\
\hline $\begin{array}{l}75-08-18 \\
75-12-09 \\
75-12-09 \\
76-03-31 \\
75-05-06\end{array}$ & $\begin{array}{r}2 \\
\text { ND } \\
-- \\
1 \\
9\end{array}$ & $\begin{array}{l}10 \\
\text { ND } \\
-- \\
10 \\
30\end{array}$ & $\begin{array}{l}40 \\
10 \\
-0 \\
10 \\
10\end{array}$ & $\begin{array}{l}20 \\
20 \\
-- \\
\text { NO } \\
40\end{array}$ \\
\hline $\begin{array}{l}75-06-19 \\
75-08-20 \\
75-12-05 \\
75-12-05 \\
76-04-01\end{array}$ & $\begin{array}{r}\text { ND } \\
\text { ND } \\
3 \\
-- \\
12\end{array}$ & $\begin{array}{l}10 \\
\text { NO } \\
\text { NO } \\
10 \\
10\end{array}$ & $\begin{array}{l}20 \\
10 \\
10 \\
-0 \\
30\end{array}$ & $\begin{array}{r}120 \\
20 \\
50 \\
-- \\
70\end{array}$ \\
\hline $\begin{array}{l}75-05-06 \\
75-06-19 \\
75-08-20 \\
75-12-05 \\
75-12-05\end{array}$ & $\begin{array}{r}\text { NO } \\
1 \\
1 \\
6 \\
--\end{array}$ & $\begin{array}{l}20 \\
\text { ND } \\
10 \\
\text { NO } \\
--\end{array}$ & $\begin{array}{r}10 \\
10 \\
170 \\
\text { ND } \\
--\end{array}$ & $\begin{array}{l}20 \\
\text { ND } \\
10 \\
\text { ND } \\
--\end{array}$ \\
\hline
\end{tabular}


water from wezls--Continued

\begin{tabular}{|c|c|c|c|c|}
\hline $\begin{array}{c}\text { DIS- } \\
\text { SOLVED } \\
\text { MERCURY } \\
\text { (HG) } \\
\text { (UG/L) }\end{array}$ & $\begin{array}{l}\text { DIS- } \\
\text { SOLVED } \\
\text { NICKEL } \\
\text { (NI) } \\
\text { (UG/L) }\end{array}$ & $\begin{array}{l}\text { DIS- } \\
\text { SOLVED } \\
\text { ZINC } \\
\text { (ZN) } \\
\text { (UG/L) }\end{array}$ & $\begin{array}{l}\text { CODE } \\
\text { FOR } \\
\text { AGENCY } \\
\text { ANA- } \\
\text { LYZING } \\
\text { SAMPLE }\end{array}$ & $\begin{array}{c}\text { TOTAL } \\
\text { DEPTH } \\
\text { OF } \\
\text { WELL } \\
\text { (FT) }\end{array}$ \\
\hline $\begin{array}{l}-\infty \\
-\infty \\
-\infty \\
-\infty \\
-\infty\end{array}$ & $\begin{array}{l}10 \\
10 \\
\text { ND } \\
--\end{array}$ & $\begin{array}{r}650 \\
-- \\
40 \\
10 \\
--\end{array}$ & $\begin{array}{l}9999 \\
9999 \\
9999 \\
9999 \\
9999\end{array}$ & $\begin{array}{r}107 \\
29 \\
29 \\
29 \\
29\end{array}$ \\
\hline $\begin{array}{l}-\infty \\
-\infty \\
-\infty \\
-\infty\end{array}$ & $\begin{array}{l}30 \\
50 \\
-- \\
\text { ND } \\
--\end{array}$ & $\begin{array}{r}50 \\
680 \\
-0 \\
80 \\
--\end{array}$ & $\begin{array}{r}9999 \\
9999 \\
9999 \\
9999\end{array}$ & $\begin{array}{l}29 \\
29 \\
29 \\
29 \\
34\end{array}$ \\
\hline $\begin{array}{l}-\infty \\
-\infty \\
-\infty \\
-\infty\end{array}$ & $\begin{array}{l}30 \\
20 \\
-0 \\
-- \\
20\end{array}$ & $\begin{array}{l}60 \\
10 \\
=- \\
-\infty\end{array}$ & $\begin{array}{l}9999 \\
9999 \\
9999 \\
9999 \\
9999\end{array}$ & $\begin{array}{l}22 \\
22 \\
22 \\
22 \\
22\end{array}$ \\
\hline $\begin{array}{l}-\infty \\
-\infty \\
-\infty \\
-\infty \\
-\infty\end{array}$ & $\begin{array}{l}\text { ND } \\
=- \\
10 \\
=-\end{array}$ & $\begin{array}{r}120 \\
210 \\
200 \\
-\infty\end{array}$ & $\begin{array}{r}9999 \\
9999 \\
-\end{array}$ & $\begin{array}{l}22 \\
22 \\
22 \\
22 \\
22\end{array}$ \\
\hline $\begin{array}{l}-\infty \\
-\infty \\
-\infty \\
-\infty\end{array}$ & $\begin{array}{l}-- \\
\text { ND } \\
30 \\
\text { NO } \\
--\end{array}$ & $\begin{array}{r}140 \\
40 \\
\text { ND } \\
-\end{array}$ & $\begin{array}{l}9999 \\
9999 \\
9999 \\
9999 \\
9999\end{array}$ & $\begin{array}{r}2101 \\
2101 \\
27 \\
27 \\
27\end{array}$ \\
\hline $\begin{array}{l}-\infty \\
-\infty \\
-\infty \\
-\infty\end{array}$ & $\begin{array}{l}30 \\
\text { ND } \\
-- \\
\text { ND } \\
40\end{array}$ & $\begin{array}{r}20 \\
60 \\
-- \\
130 \\
N D\end{array}$ & $\begin{array}{r}9999 \\
9999 \\
-- \\
9999 \\
9999\end{array}$ & $\begin{array}{r}27 \\
27 \\
27 \\
27 \\
130\end{array}$ \\
\hline $\begin{array}{l}-\infty \\
-\infty \\
-\infty \\
-\infty\end{array}$ & $\begin{array}{l}10 \\
10 \\
\text { ND } \\
-10\end{array}$ & $\begin{array}{l}10 \\
10 \\
50 \\
-- \\
70\end{array}$ & $\begin{array}{r}9999 \\
9999 \\
9999 \\
-0 \\
9999\end{array}$ & $\begin{array}{l}130 \\
130 \\
130 \\
130 \\
130\end{array}$ \\
\hline $\begin{array}{l}-- \\
-- \\
--\end{array}$ & $\begin{array}{l}10 \\
40 \\
\text { ND } \\
\text { ND } \\
--\end{array}$ & $\begin{array}{r}\text { ND } \\
30 \\
160 \\
30 \\
-\end{array}$ & $\begin{array}{r}9999 \\
9999 \\
9999 \\
9999 \\
\end{array}$ & $\begin{array}{l}175 \\
175 \\
175 \\
175 \\
175\end{array}$ \\
\hline
\end{tabular}


Table 5.--Chemical analyses of

LOCAL

IDENT-

I-

FIER

SC005065068DD2
SC005065068D03

SC00506506CACl

SC00506506CDA

SC00506506CDC

SC00506506CDO

SC00506506CAC2

$\begin{array}{cccc} & \text { TOTAL } & \text { DIS- } & \text { DIS- } \\ \text { DATE } & \text { DEPTH } & \text { SOLVED } & \text { SOLVED } \\ \text { OF } & \text { OF } & \text { SILICA } & \text { IRON } \\ \text { SAMPLE } & \text { WELL } & (\text { SIOZ) } & \text { (FE) } \\ & (F T) & (M G / L) & (U G / L)\end{array}$

76-04-01 175

76-04-01 175

$74-11-26 \quad 37$

75-02-12 37

75-03-20 37

75-05-06 37

$75-08-14 \quad 37$

$75-12-05 \quad 37$

$75-12-05 \quad 37$

76-03-30 37

76-04-16 37

75-06-19 53

75-08-20 53

75-12-05 53

75-12-05

53

75-06-19 153

$75-08-20 \quad 153$

75-12-05 153

$75-12-05 \quad 153$

$74-11-26 \quad 63$

$75-02-12$

$75-03-20$

75-05-07

$75-08-14$

$75-12-04$

75-12-04

76-03-30

$74-11-26$

$75-02-11$

75-03-20

63

63

63

63

63

63

63

150

150

150

75-05-06

$75=08=14$

$75-12=05$

$75-12-05$

76-04-01

150

150

150

150

150

76-04-16 150

$74-11-27$

$75-02-11$

$75-03-20$

75-05-07
53

53

53

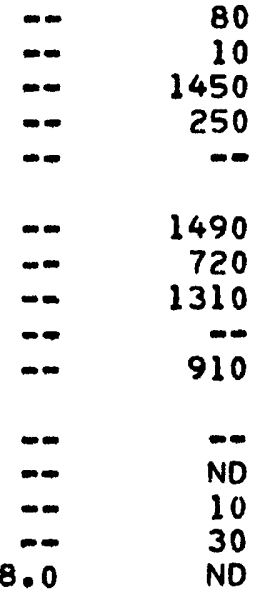

$\ldots \quad$ ND

-- 20

- 40

$-\infty \quad-\infty$

$-\infty \quad 720$

$\ldots \quad 3300$

--

$=1400$

$-\infty \quad 1300$

$=\quad 6900$

- 20

-- 710

$\ldots$

- 3200

-. 320

-- 980

17

950

1030

$\begin{array}{ll}-\infty & 30 \\ -\infty & 50 \\ -\infty & 30\end{array}$ $-\infty \quad 2200$ 
water from wells--Continued

$\begin{array}{cccccc} & & \text { DIS- } & & \text { DIS- } \\ \text { OIS- } & \text { OIS- } & \text { SOLVED } & & \text { SOLVED } & \\ \text { SOLVED } & \text { SOLVED } & \text { MAG- } & \text { DIS- } & \text { PO- } & \\ \text { MAN- } & \text { CAL- } & \text { NE- } & \text { SOLVED } & \text { TAS- } & \text { BICAR- } \\ \text { GANESE } & \text { CIUM } & \text { SIUM } & \text { SODIUM } & \text { SIUM } & \text { BONATE } \\ \text { (MN) } & \text { (CA) } & \text { (MG) } & \text { (NA) } & \text { (K) } & \text { (HCO3) } \\ (U G / L) & (M G / L) & \text { (MG/L) } & \text { (MG/L) } & (M G / L) & \text { (MG/L) }\end{array}$

\begin{tabular}{|c|c|c|c|c|c|}
\hline $\begin{array}{r}250 \\
20 \\
200 \\
2260\end{array}$ & $\begin{array}{l}26^{--} \\
450 \\
212\end{array}$ & $\begin{array}{l}1.3 \\
=- \\
=-\end{array}$ & $\begin{array}{l}70^{-} \\
155 \\
185\end{array}$ & $\begin{array}{c}6.0 \\
22.0 \\
6.5\end{array}$ & $\begin{array}{l}-113 \\
113 \\
366 \\
387\end{array}$ \\
\hline-- & $-\infty$ & $-\infty$ & $-\infty$ & $\infty$ & - \\
\hline $\begin{array}{l}2880 \\
3800\end{array}$ & $\begin{array}{l}227 \\
107 \\
176\end{array}$ & $=$ & $\begin{array}{l}190 \\
230 \\
200\end{array}$ & $19^{--}$ & $\begin{array}{r}-5 \\
357 \\
360\end{array}$ \\
\hline 3900 & $122^{-\infty}$ & $\begin{array}{l}17 \\
20\end{array}$ & $195^{--}$ & $13^{--}$ & 363 \\
\hline $\begin{array}{l}10 \\
10 \\
10 \\
\text { ND } \\
\text { ND }\end{array}$ & $\begin{array}{l}314^{--} \\
442 \\
222 \\
240\end{array}$ & $\begin{array}{l}= \\
-\infty \\
-1\end{array}$ & $\begin{array}{r}97^{--} \\
94 \\
142^{--} \\
140^{-}\end{array}$ & $\begin{array}{l}8.0 \\
16 \\
20 \\
9.8\end{array}$ & $\begin{array}{l}-\ddot{n} \\
\text { ND } \\
\text { ND } \\
\text { ND }\end{array}$ \\
\hline $\begin{array}{l}\text { ND } \\
30 \\
\text { ND }\end{array}$ & $\begin{array}{l}478 \\
683 \\
510\end{array}$ & $-\infty$ & $\begin{array}{r}180 \\
94 \\
168\end{array}$ & $\begin{array}{c}120 \\
8.0 \\
42\end{array}$ & $\begin{array}{l}\text { ND } \\
\text { ND } \\
\text { ND }\end{array}$ \\
\hline 2350 & $360^{-\infty}$ & ND & $280^{--}$ & $19^{-\infty}$ & 1170 \\
\hline 8500 & 324 & - & 260 & 9.5 & 397 \\
\hline $\begin{array}{l}5200 \\
4800\end{array}$ & $\begin{array}{l}404 \\
265 \\
362\end{array}$ & $\begin{array}{l}-\infty \\
-\infty \\
-\infty\end{array}$ & $\begin{array}{l}210^{--} \\
200 \\
260\end{array}$ & $\begin{array}{l}19^{-} \\
23^{-}\end{array}$ & $\begin{array}{l}-0 \\
362 \\
377\end{array}$ \\
\hline $\begin{array}{r}12500 \\
340 \\
310\end{array}$ & $\begin{array}{l}902^{-\infty} \\
188 \\
246\end{array}$ & $\begin{array}{r}63 \\
34 \\
\ldots\end{array}$ & $\begin{array}{l}1220^{-0} \\
150 \\
200\end{array}$ & $\begin{array}{l}34^{--} \\
15 \\
7.4\end{array}$ & $\begin{array}{l}-1 \\
393 \\
316 \\
302\end{array}$ \\
\hline$-\infty$ & $-\infty$ & $-\infty$ & $-\infty$ & $-\infty$ & - \\
\hline $\begin{array}{l}220 \\
390 \\
490 \\
480\end{array}$ & $\begin{array}{l}222 \\
125 \\
140 \\
280 \\
221\end{array}$ & $\begin{array}{l}28^{--} \\
25\end{array}$ & $\begin{array}{l}130 \\
130 \\
163 \\
160 \\
192\end{array}$ & $\begin{array}{l}20^{-0} \\
16 \\
9.2 \\
15^{-2}\end{array}$ & $\begin{array}{l}-0 \\
288 \\
288 \\
371 \\
267\end{array}$ \\
\hline $\begin{array}{r}850 \\
1280\end{array}$ & $\begin{array}{l}270^{-} \\
343\end{array}$ & $=$ & $\begin{array}{l}120^{--} \\
135\end{array}$ & $\begin{array}{l}18^{--} \\
11\end{array}$ & $\begin{array}{l}209 \\
260\end{array}$ \\
\hline$-\infty$ & $408^{--}$ & $=-$ & $130^{-\infty}$ & $-\infty$ & $-\infty$ \\
\hline
\end{tabular}


Table 5.--Chemical analyses of

\begin{tabular}{|c|c|c|c|c|c|}
\hline $\begin{array}{c}\text { DATE } \\
\text { OF } \\
\text { SAMPLE }\end{array}$ & $\begin{array}{l}\text { CAR- } \\
\text { BONATE } \\
(C O 3) \\
(M G / L)\end{array}$ & $\begin{array}{c}H Y- \\
D R O X- \\
I D E \\
(O H) \\
(M G / L)\end{array}$ & $\begin{array}{l}\text { ALKA- } \\
\text { LINITY } \\
\text { AS } \\
\text { CACO3 } \\
\text { (MG/L) }\end{array}$ & $\begin{array}{l}\text { DIS- } \\
\text { SOLVED } \\
\text { SULFATE } \\
\text { (SO4) } \\
\text { (MG/L) }\end{array}$ & $\begin{array}{l}\text { DIS- } \\
\text { SOLVEO } \\
\text { CHLO- } \\
\text { RIDE } \\
\text { (CL) } \\
(M G / L)\end{array}$ \\
\hline $\begin{array}{l}76-04-01 \\
76-04-01 \\
74-11-26 \\
75-02-12 \\
75-03-20\end{array}$ & $\begin{array}{l}-- \\
\text { ND } \\
\text { ND } \\
-- \\
--\end{array}$ & $\begin{array}{l}-- \\
\text { NO } \\
-- \\
--\end{array}$ & $\begin{array}{r}-\infty \\
93 \\
300 \\
317 \\
--\end{array}$ & $24^{-\infty}$ & $\begin{array}{l}54^{--} \\
31 \\
28 \\
\end{array}$ \\
\hline $\begin{array}{l}75-05-06 \\
75-08-14 \\
75-12-05 \\
75-12-05 \\
76-03-30\end{array}$ & $\begin{array}{l}-\infty \\
\text { ND } \\
\text { ND } \\
-- \\
\text { ND }\end{array}$ & $\begin{array}{l}-- \\
\text { ND } \\
\text { ND } \\
-- \\
\text { ND }\end{array}$ & $\begin{array}{r}-- \\
293 \\
295 \\
-- \\
298\end{array}$ & $\begin{array}{l}720^{--} \\
680^{--} \\
562^{--}\end{array}$ & $\begin{array}{l}2.9 \\
42 \\
34 \\
57\end{array}$ \\
\hline $\begin{array}{l}76-04-16 \\
75-06-19 \\
75-08-20 \\
75-12-05 \\
75-12-05\end{array}$ & $\begin{array}{l}-- \\
60 \\
40 \\
80 \\
\text { ND }\end{array}$ & $\begin{array}{r}-0 \\
990 \\
1060 \\
420 \\
130\end{array}$ & $\begin{array}{l}= \\
-0 \\
-0\end{array}$ & $\begin{array}{l}390 \\
275 \\
530 \\
470\end{array}$ & $\begin{array}{l}21 \\
27 \\
26 \\
22\end{array}$ \\
\hline $\begin{array}{l}75-06-19 \\
75-08-20 \\
75-12-05 \\
75-12-05 \\
74-11-26\end{array}$ & $\begin{array}{r}4860 \\
180 \\
2360 \\
-- \\
\text { ND }\end{array}$ & $\begin{array}{r}3930 \\
2010 \\
640 \\
-0 \\
-\end{array}$ & $\begin{array}{l}- \\
-0 \\
-\infty \\
960\end{array}$ & $\begin{array}{l}23^{N D} \\
20 \\
=-\end{array}$ & $\begin{array}{l}13 \\
22 \\
30 \\
69^{--}\end{array}$ \\
\hline $\begin{array}{l}75-02-12 \\
75-03-20 \\
75-05-07 \\
75-08-14 \\
75-12-04\end{array}$ & $\begin{array}{l}-- \\
-\infty \\
\text { ND } \\
\text { ND }\end{array}$ & $\begin{array}{l}-- \\
-- \\
\text { ND } \\
\text { ND }\end{array}$ & $\begin{array}{r}326 \\
-- \\
-- \\
297 \\
309\end{array}$ & $415^{-\infty}$ & $\begin{array}{l}596 \\
600 \\
641 \\
653\end{array}$ \\
\hline $\begin{array}{l}75-12-04 \\
76-03-30 \\
74-11-26 \\
75-02-11 \\
75-03-20\end{array}$ & $\begin{array}{l}-- \\
\text { ND } \\
\text { ND } \\
-- \\
--\end{array}$ & $\begin{array}{l}-- \\
-- \\
--\end{array}$ & $\begin{array}{r}-- \\
322 \\
259 \\
248 \\
--\end{array}$ & $341^{--}$ & $\begin{array}{c}3050 \\
41 \\
70\end{array}$ \\
\hline $\begin{array}{l}75-05-06 \\
75-08-14 \\
75-12-05 \\
75-12-05 \\
76-04-01\end{array}$ & $\begin{array}{l}-- \\
\text { ND } \\
\text { ND } \\
\text { ND } \\
\text { ND }\end{array}$ & $\begin{array}{l}-- \\
\text { NO } \\
\text { NO } \\
-- \\
\text { NO }\end{array}$ & $\begin{array}{l}-- \\
236 \\
236 \\
304 \\
219\end{array}$ & $\begin{array}{l}820^{--} \\
480 \\
730 \\
666\end{array}$ & $\begin{array}{l}38 \\
80 \\
64 \\
61 \\
46\end{array}$ \\
\hline $\begin{array}{l}76-04-16 \\
74-11-27 \\
75-02-11 \\
75-03-20 \\
75-05-07\end{array}$ & $\begin{array}{l}-- \\
\text { ND } \\
-- \\
--\end{array}$ & $\begin{array}{l}\ldots \\
-- \\
--\end{array}$ & $\begin{array}{r}-0 \\
171 \\
213 \\
-\infty \\
-\infty\end{array}$ & $\begin{array}{l}-\infty \\
-\infty \\
-\infty \\
-\infty\end{array}$ & $\begin{array}{l}48^{--} \\
29^{--} \\
46^{--}\end{array}$ \\
\hline
\end{tabular}


water from wells--Continued

DIS-

SOLVED

FLUO-

RIDE

(F)

DISSOLVED NITRATE

(N)

(MG/L)

TOTAL SOLVED SOLVED SOLVED NITRATE NITRATE NITRITE (NO3) (NO3)

(N)

(MG/L) (MG/L)
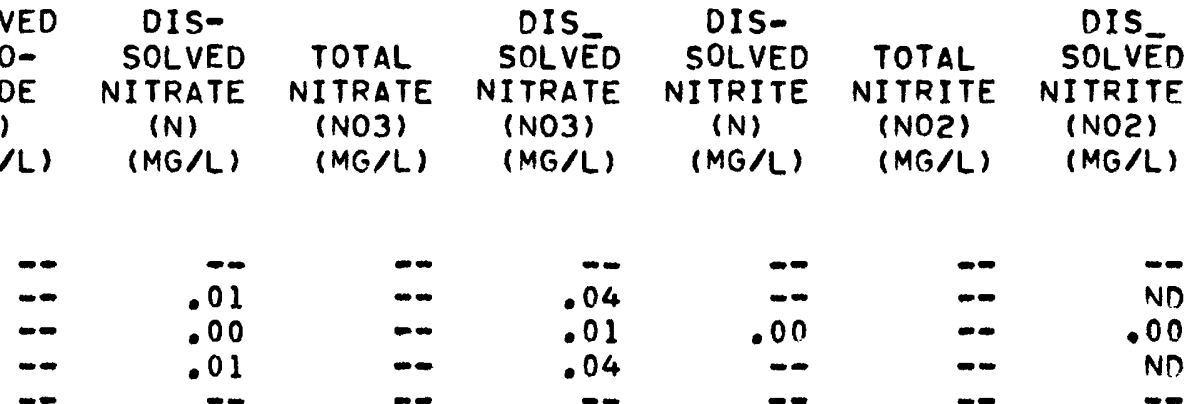

.00

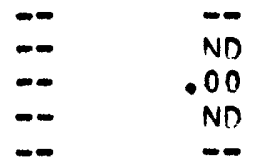

-02
$-\quad .02$

-. $\quad .00$

--

$\ldots .01$

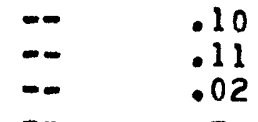

$\because$

$-$

$\because$

.05

.00

$-$

$\begin{array}{ll}\ldots & \text { Nn } \\ \ldots & \text { Nก }\end{array}$

$\because \quad$ ND

$-05$

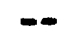

.01

$=\quad$ ND

$-$

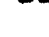

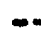

$-\infty .02$

$-\infty \quad .05$

.00

- NN

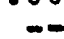

.01

.02

-. 10

$\because$

$-$

ND

$.3 \quad .03$

$\begin{array}{ll}-0 & .03 \\ -\infty & .04\end{array}$

.00

-

.03

$\begin{array}{ll}-0 & 01 \\ - & 01\end{array}$

$-$

$-0.02$

$\because$

.02

.00

-

$-\infty$

$=-.01$

$\infty .00$

-

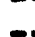

ND

$\ldots .00$

$-$

.00

$\ldots .02$

-. .26

$-$

.01

-- $\quad .16$

$-09$

-

$-.72$

$\ldots \quad .40$

.03

$-.06$

$-\infty$

$\ldots .10$

$-. .12$

-. .52

$=$

ND

$\begin{array}{ll}-\infty & -26\end{array}$

$\ldots \quad .03$

$-$

NO

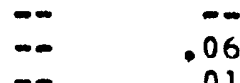

$\ldots \quad .01$

$-\infty .08$

.0

$-$

$\infty$

$\ddot{--}$

Nก

.00

.01

-.

$-$

$-\infty$

$-.02$

$\begin{array}{ll}-0 & 00 \\ -\infty & 0\end{array}$

$\begin{array}{ll}-0 & .01 \\ -0 & .46\end{array}$

-- .11

.2 .50

- $\quad .47$

- 2.2

.05

-. .20

$\because$

-:

ND

$-\quad$ NO

No

-

ND

$\ldots \quad 5.4$

-

- 3.0

$\ldots \quad 3.0$

$\begin{array}{ll}-\infty & 23 \\ -\infty & 13\end{array}$

.14

.02

.00

$-\infty$

$--\quad$ ND

$-\infty 17^{-\infty}$

$\begin{array}{lr}- & -5 \\ -- & .46 \\ -0 & .07 \\ - & -0\end{array}$ 
Table 5.--Chemical analyses of

\begin{tabular}{|c|c|c|c|c|c|}
\hline $\begin{array}{c}\text { DATE } \\
\text { OF } \\
\text { SAMPLE }\end{array}$ & $\begin{array}{l}\text { DIS- } \\
\text { SOLVED } \\
\text { AMMONIA } \\
\text { NITRO- } \\
\text { GEN } \\
\text { (N) } \\
(M G / L)\end{array}$ & $\begin{array}{l}\text { TOTAL } \\
\text { AMMONIA } \\
\text { (NH4) } \\
\text { (MG/L) }\end{array}$ & $\begin{array}{l}\text { DIS } \\
\text { SOLVED } \\
\text { AMMONIA } \\
\text { (NH4) } \\
(M G / L)\end{array}$ & $\begin{array}{l}\text { DIS- } \\
\text { SOLVED } \\
\text { ORGANIC } \\
\text { NITRO- } \\
\text { GEN } \\
\text { (N) } \\
\text { (MG/L) }\end{array}$ & $\begin{array}{l}\text { DIS- } \\
\text { SOLVED } \\
\text { KJEL. } \\
\text { NITRO- } \\
\text { GEN } \\
\text { (N) } \\
\text { (MG/L) }\end{array}$ \\
\hline $\begin{array}{l}76-04-01 \\
76-04-01 \\
74-11-26 \\
75-02-12 \\
75-03-20\end{array}$ & $\begin{array}{l}-- \\
\text { NO } \\
\text { ND } \\
\text { ND } \\
--\end{array}$ & $\begin{array}{l}-\infty \\
=- \\
-\infty \\
-\infty\end{array}$ & $\begin{array}{l}-\infty \\
-\infty \\
-\infty \\
-\infty\end{array}$ & $\begin{array}{l}-\infty \\
-\infty \\
-\infty\end{array}$ & $\begin{array}{l}-- \\
\text { NO } \\
1.6 \\
.90 \\
-\infty\end{array}$ \\
\hline $\begin{array}{l}75-05-06 \\
75-08-14 \\
75-12-05 \\
75-12-05 \\
76-03-30\end{array}$ & $\begin{array}{l}.10 \\
.20 \\
.10 \\
.10\end{array}$ & $\begin{array}{l}-\infty \\
=- \\
-\infty \\
-\infty\end{array}$ & $\begin{array}{r}.13 \\
.26 \\
.13 \\
.13 \\
.13\end{array}$ & $\begin{array}{r}.50 \\
1.2 \\
.40 \\
.0 \\
.40\end{array}$ & $\begin{array}{l}.60 \\
1.4 \\
.50 \\
.50 \\
.50\end{array}$ \\
\hline $\begin{array}{l}76-04-16 \\
75-06-19 \\
75-08-20 \\
75-12-05 \\
75-12-05\end{array}$ & $\begin{array}{r}.10 \\
\text { ND } \\
.60 \\
.18\end{array}$ & $\begin{array}{l}-- \\
-- \\
=- \\
=-\end{array}$ & $\begin{array}{r}.0- \\
.13 \\
.77 \\
.73\end{array}$ & $\begin{array}{r}.-10 \\
.10 \\
-2 \\
.20 \\
.72\end{array}$ & $\begin{array}{l}.2 \\
.20 \\
.90 \\
.80 \\
.90\end{array}$ \\
\hline $\begin{array}{l}75-06-19 \\
75-08-20 \\
75-12-05 \\
75-12-05 \\
74-11-26\end{array}$ & $\begin{array}{l}1.8 \\
1.4 \\
2.3 \\
-.\end{array}$ & $\begin{array}{l}= \\
=- \\
=- \\
=-\end{array}$ & $\begin{array}{c}2.3 \\
1.8 \\
3.0 \\
=-\end{array}$ & $\begin{array}{l}1.1 \\
2.3 \\
.40 \\
=- \\
=\end{array}$ & $\begin{array}{c}2.9 \\
3.7 \\
2.7 \\
12^{--}\end{array}$ \\
\hline $\begin{array}{l}75-02-12 \\
75-03-20 \\
75-05-07 \\
75-08-14 \\
75-12-04\end{array}$ & $\begin{array}{r}.10 \\
.-1 \\
.10 \\
.20 \\
\text { NO }\end{array}$ & $\begin{array}{l}=- \\
=- \\
=- \\
=-\end{array}$ & $\begin{array}{r}.13 \\
.0 \\
.13 \\
.26 \\
.-\end{array}$ & $\begin{array}{r}.60 \\
.50 \\
.5 \\
.-\end{array}$ & $\begin{array}{r}.70 \\
.60 \\
\text { ND } \\
.60\end{array}$ \\
\hline $\begin{array}{l}75-12-04 \\
76-03-30 \\
74-11-26 \\
75-02-11 \\
75-03-20\end{array}$ & $\begin{array}{r}-- \\
\text { NO } \\
\text { NO } \\
.10 \\
--\end{array}$ & $\begin{array}{l}=- \\
=- \\
=- \\
-\infty\end{array}$ & $\begin{array}{l}-- \\
=- \\
13 \\
--\end{array}$ & $\begin{array}{l}-- \\
- \\
.30 \\
--\end{array}$ & $\begin{array}{l}.- \\
.70 \\
.20 \\
.40 \\
-\infty\end{array}$ \\
\hline $\begin{array}{l}75-05-06 \\
75-08-14 \\
75-12-05 \\
75-12-05 \\
76-04-01\end{array}$ & $\begin{array}{l}.20 \\
.10 \\
.10 \\
.14 \\
\text { ND }\end{array}$ & $\begin{array}{l}=- \\
=- \\
=- \\
--\end{array}$ & $\begin{array}{r}.26 \\
.13 \\
.13 \\
.18 \\
.-\end{array}$ & $\begin{array}{l}-\infty \\
. \\
.30 \\
.25 \\
--\end{array}$ & $\begin{array}{r}.10 \\
\text { ND } \\
.40 \\
.39 \\
.30\end{array}$ \\
\hline $\begin{array}{l}76-04-16 \\
74-11-27 \\
75-02-11 \\
75-03-20 \\
75-05-07\end{array}$ & $\begin{array}{l}-- \\
\text { ND } \\
\text { ND } \\
-- \\
.10\end{array}$ & $\begin{array}{l}-- \\
-- \\
--\end{array}$ & $\begin{array}{l}= \\
=- \\
=- \\
13\end{array}$ & $\begin{array}{l}=- \\
= \\
= \\
.40\end{array}$ & $\begin{array}{r}.60 \\
.70 \\
.50\end{array}$ \\
\hline
\end{tabular}


water from wells--Continued

$\begin{array}{clccccc}\text { OIS- } & \text { DIS- } & \text { DIS- } & \text { DIS- } & \text { DIS- } & & \\ \text { SOLVED } & \text { SOLVED } & \text { SOLVED } & \text { SOLVED } & \text { SOLVED } & & \text { NON- } \\ \text { ORTHO. } & \text { ORTHO } & \text { SOLIDS } & \text { SOLIDS } & \text { SOLIDS } & & \text { CAR- } \\ \text { PHOS- } & \text { PHOS- } & \text { (RESI- } & \text { (RESI- } & \text { (SUM OF } & \text { HARD- } & \text { BONATE } \\ \text { PHORUS } & \text { PHATE } & \text { DUE AT } & \text { DUE AT } & \text { CONSTI- } & \text { NESS } & \text { HARD- } \\ (P) & (P O 4) & \left.180^{\circ} C\right) & \left.105^{\circ} C\right) & \text { TUENTS) } & \text { (CA,MG) } & \text { NESS } \\ (M G / L) & (M G / L) & (M G / L) & (M G / L) & (M G / L) & (M G / L) & \text { (MG/L) }\end{array}$

\begin{tabular}{|c|c|c|c|c|c|c|}
\hline $\begin{array}{l}-\infty \\
1.0 \\
1.0\end{array}$ & $\begin{array}{l}-- \\
3.1 \\
5.5\end{array}$ & $\begin{array}{l}-\infty \\
-\infty \\
-\infty\end{array}$ & $\begin{array}{r}309 \\
2230 \\
1520\end{array}$ & $\begin{array}{l}-\infty \\
-\infty \\
-\infty\end{array}$ & $\begin{array}{r}51 \\
51 \\
1220 \\
644\end{array}$ & $\begin{array}{r}0 \\
920 \\
330\end{array}$ \\
\hline-- & -- & -- & - & - & -- & - \\
\hline $\begin{array}{r}-\infty \\
\text { ND } \\
.01\end{array}$ & .03 & $=$ & $\begin{array}{l}1450 \\
1520 \\
1180\end{array}$ & $=$ & $\begin{array}{l}693 \\
612\end{array}$ & $\begin{array}{l}-0 \\
400 \\
320\end{array}$ \\
\hline$\ddot{\text { ND }}$ & $-\infty$ & $-\infty$ & 1400 & 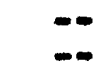 & 594 & 300 \\
\hline $\begin{array}{l}-0 \\
.02 \\
\text { ND } \\
\text { ND } \\
\text { ND }\end{array}$ & $\begin{array}{r}.06 \\
.0 \\
-\infty \\
\text { NO }\end{array}$ & $\begin{array}{l}-\infty \\
-\infty \\
-\infty\end{array}$ & $\begin{array}{r}1650 \\
1550 \\
1280 \\
\end{array}$ & 1020 & $\begin{array}{r}1190 \\
1190 \\
560 \\
600\end{array}$ & $\begin{array}{l}-0 \\
00 \\
000\end{array}$ \\
\hline $\begin{array}{l}.03 \\
\text { ND } \\
\text { ND }\end{array}$ & $\begin{array}{l}.09 \\
- \\
-\end{array}$ & $=$ & $\begin{array}{l}2130 \\
1990 \\
1830\end{array}$ & $=$ & $\begin{array}{l}1670 \\
1600 \\
1410\end{array}$ & $=$ \\
\hline $7.5^{--}$ & $23^{--}$ & $=$ & 2380 & $=$ & 1210 & 250 \\
\hline $\begin{array}{r}.80 \\
-- \\
-0 \\
\text { NO } \\
.01\end{array}$ & $\begin{array}{r}2.5 \\
- \\
-0 \\
.03\end{array}$ & $\begin{array}{l}- \\
- \\
- \\
- \\
-\end{array}$ & $\begin{array}{r}2370 \\
2190 \\
2210 \\
2400\end{array}$ & $\begin{array}{l}= \\
-- \\
-\end{array}$ & $\begin{array}{r}1060 \\
- \\
1090 \\
1270\end{array}$ & $\begin{array}{r}730 \\
-0 \\
-0 \\
790 \\
960\end{array}$ \\
\hline $\begin{array}{r}-- \\
\text { ND } \\
.06 \\
\text { ND }\end{array}$ & $\begin{array}{l}-0 \\
.18 \\
-\end{array}$ & $=$ & $\begin{array}{l}7110 \\
1510 \\
1620\end{array}$ & $\ddot{-\infty}$ & $\begin{array}{r}3870 \\
755 \\
894\end{array}$ & $\begin{array}{r}-0 \\
3500 \\
500 \\
650\end{array}$ \\
\hline-- & $\cdots$ & - & $-\infty$ & $-\infty$ & - & $\infty$ \\
\hline $\begin{array}{r}- \\
\text { ND } \\
.01 \\
.01 \\
\text { ND }\end{array}$ & $\begin{array}{r}- \\
-0 \\
.03 \\
.03 \\
-\end{array}$ & $\begin{array}{l}-\infty \\
=- \\
-\infty\end{array}$ & $\begin{array}{r}1540 \\
1680 \\
1610 \\
1450\end{array}$ & 1470 & $\begin{array}{l}-0 \\
849 \\
830 \\
810 \\
691\end{array}$ & $\begin{array}{l}-0 \\
510 \\
590 \\
510 \\
470\end{array}$ \\
\hline $\begin{array}{r}-- \\
.40 \\
\text { ND }\end{array}$ & $1.2^{--}$ & $=$ & $\begin{array}{l}1960 \\
2310\end{array}$ & $=$ & $\begin{array}{l}1150 \\
1270\end{array}$ & $\begin{array}{r}980 \\
1100\end{array}$ \\
\hline$\because$ & $\therefore$ & 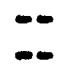 & 2070 & 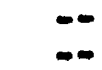 & $=$ & $=$ \\
\hline
\end{tabular}




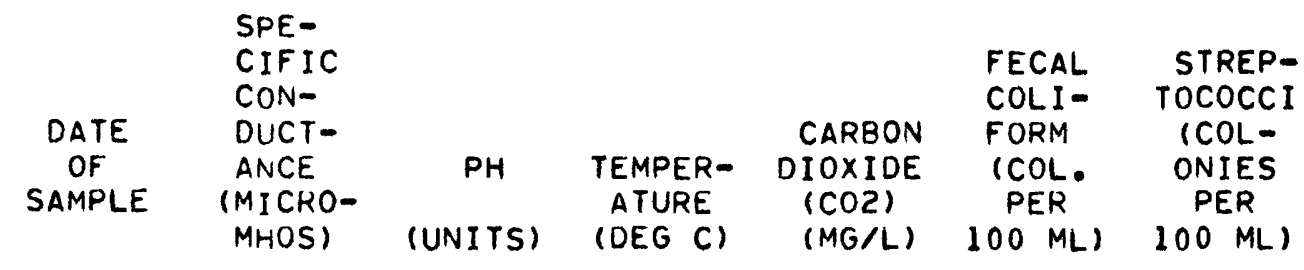

$\begin{array}{rr}76-04-01 & 460 \\ 76-04-01 & 460 \\ 74-11-26 & 2900 \\ 75-02-12 & 2175 \\ 75-03-20 & -- \\ & \\ 75-05-06 & 2100 \\ 75-08-14 & 2500 \\ 75-12-05 & 2100 \\ 75-12-05 & 2100 \\ 76-03-30 & 1650 \\ 76-04-16 & \\ 75-06-19 & 7500 \\ 75-08-20 & 8000 \\ 75-12-05 & 3900 \\ 75-12-05 & 3900 \\ & \\ 75-06-19 & >8000 \\ 75-08-20 & >8000 \\ 75-12-05 & >8000 \\ 75-12-05 & >8000 \\ 74-11-26 & 4000 \\ 75-02-12 & 3500 \\ 75-03-20 & -- \\ 75-05-07 & 3900 \\ 75-08-14 & 4500 \\ 75-12-04 & 4000 \\ 75-12-04 & 4000 \\ 76-03-30 & >8000 \\ 74-11-26 & 2000 \\ 75-02-11 & 2100 \\ 75-03-20 & -- \\ 75-05-06 & 2200 \\ 75-08-14 & 2500 \\ 75-12-05 & 2600 \\ 75-12-05 & 2600 \\ 76-04-01 & 1800 \\ 76-04-16 & -- \\ 74-11-27 & 2400 \\ 75-02-11 & 2800 \\ 75-03-20 & -- \\ 75-05-07 & 2800\end{array}$

\begin{tabular}{|c|c|c|c|}
\hline $\begin{array}{l}8.0 \\
8.0 \\
6.9 \\
6.8\end{array}$ & $\begin{array}{l}15.0 \\
15.0 \\
10.5 \\
11.0\end{array}$ & $\begin{array}{l}1.8 \\
74 \\
98\end{array}$ & $\begin{array}{c}-- \\
-- \\
0\end{array}$ \\
\hline-- & -- & - & $-\infty$ \\
\hline $\begin{array}{l}6.9 \\
7.2 \\
7.6 \\
7.6 \\
7.3\end{array}$ & $\begin{array}{r}11.5 \\
13.5 \\
8.5 \\
8.5 \\
11.0\end{array}$ & $\begin{array}{l}36^{--} \\
14^{--} \\
29^{--}\end{array}$ & $\begin{array}{l}=- \\
-- \\
--\end{array}$ \\
\hline
\end{tabular}

$\begin{array}{lllll}11.2 & 13.5 & -- & -\infty & -- \\ 11.5 & 14.0 & -- & -- & - \\ 11.9 & 10.0 & -- & -- & - \\ 11.9 & 10.0 & -- & -\infty & -\end{array}$

$12.3 \quad 14.0$

$11.6 \quad 14.0$

$11.9 \quad 12.0$

$11.9 \quad 12.0$

6.515 .0

$6.6 \quad 18.5$

$7.0 \quad 19.0$

$6.9 \quad 21.5$

$7.1 \quad 19.0$

$7.1 \quad 19.0$

6.816 .0

$6.9 \quad 9.5$

6.910 .0

0.0

$6.9 \quad 11.5$

$7.3 \quad 14.5$

$7.1 \quad 12.5$

$7.1 \quad 12.5$

$7.1 \quad 14.0$

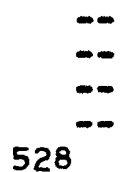

$-$

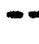

$-$

$\overline{-}$

160

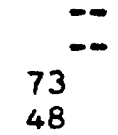

$\begin{array}{ll}0 & 1 \\ -\infty & -\infty \\ -\infty & -\infty\end{array}$

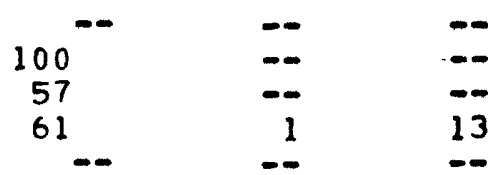

$6.6 \quad 11.0$

$6.7 \quad 10.0$

6.7

10.5

23
37
47
34
$84^{-0}$
8

$=-$
$=-$
$=$
$=-$
$=-$ 
water from welzs--Continued

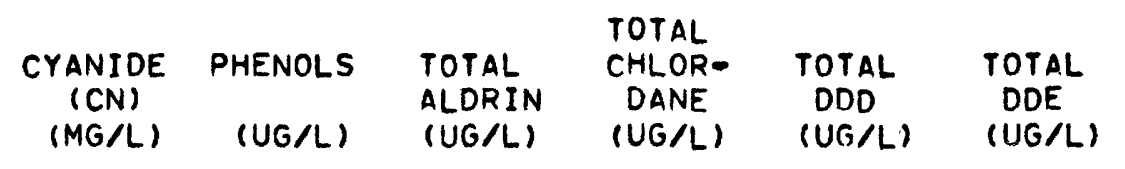

\begin{tabular}{|c|c|c|c|c|c|}
\hline$-\infty$ & $-\infty$ & $-\infty$ & $-\infty$ & -- & - \\
\hline$-\infty$ & $-\infty$ & -- & $-\infty$ & $=-$ & $\infty$ \\
\hline- & $-\infty$ & $=-$ & $-\infty$ & $-\infty$ & - \\
\hline ND & -- & $-\infty$ & $-\infty$ & $-\infty$ & - \\
\hline-- & 220 & $-\infty$ & -- & $\infty$ & $-\infty$ \\
\hline$-\infty$ & $-\infty$ & $-\infty$ & $-\infty$ & $-\infty$ & - \\
\hline$-\infty$ & - & ND & ND & ND & ND \\
\hline$-\infty$ & -- & $-\infty$ & -- & $-\infty$ & $-\infty$ \\
\hline$-\infty$ & - & $-\infty$ & $-\infty$ & $-\infty$ & $-\infty$ \\
\hline$-\infty$ & $-\infty$ & $-\infty$ & $-\infty$ & -- & - \\
\hline$-\infty$ & $-\infty$ & $-\infty$ & $-\infty$ & -- & $-\infty$ \\
\hline- & -- & $-\infty$ & -- & $-\infty$ & $m$ \\
\hline$-\infty$ & $-\infty$ & ND & ND & ND & ND \\
\hline$-\infty$ & - & $-\infty$ & - & - & $-\infty$ \\
\hline-- & $-\infty$ & - & $-\infty$ & $-\infty$ & $-\infty$ \\
\hline- & $-\infty$ & $-\infty$ & $-\infty$ & $\infty$ & $\rightarrow$ \\
\hline$=$ & $-\infty$ & 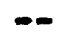 & $-\infty$ & $-\infty$ & \\
\hline$=$ & $-\infty$ & $-\infty$ & $-\infty$ & $-\infty$ & - \\
\hline-- & $-\infty$ & - & $-\infty$ & -- & - \\
\hline$=$ & $-\infty$ & -- & 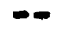 & -- & סי \\
\hline ND & $-\infty$ & $-\infty$ & $-\infty$ & $-\infty$ & - \\
\hline$m-$ & ND & $-\infty$ & $-\infty$ & $-\infty$ & $-\infty$ \\
\hline$-\infty$ & $\infty$ & $-\infty$ & $-\infty$ & $-\infty$ & - \\
\hline$-\infty$ & $-\infty$ & $-\infty$ & $-\infty$ & $-\infty$ & - \\
\hline$-\infty$ & $-\infty$ & - & $-\infty$ & $\cdots$ & $-\infty$ \\
\hline$m-\infty$ & $-\infty$ & $-\infty$ & $-\infty$ & $\Rightarrow$ & 10 \\
\hline$-\infty$ & - & $-\infty$ & $-\infty$ & - & - \\
\hline$-\infty$ & $-\infty$ & $-\infty$ & $-\infty$ & $-\infty$ & 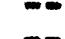 \\
\hline$=$ & $\overline{N D}$ & $=$ & $=$ & $-\infty$ & - \\
\hline$-\infty$ & ND & - & $-\infty$ & $-\infty$ & $-\infty$ \\
\hline- & $-\infty$ & -- & -- & $-\infty$ & - \\
\hline$\cdots$ & $-\infty$ & $-\infty$ & -- & $-\infty$ & \\
\hline$-\infty$ & $-\infty$ & -- & -- & $\infty$ & \\
\hline$-\infty$ & $\cdots$ & $-\infty$ & $-\infty$ & $-\infty$ & \\
\hline$=$ & -- & -- & $-\infty$ & $-\infty$ & \\
\hline-- & $-\infty$ & -- & $\infty$ & $\infty$ & \\
\hline$-\infty$ & $-\infty$ & - & $-\infty$ & $-\infty$ & \\
\hline ND & -- & -- & - & $-\infty$ & - \\
\hline$=$ & ND & $=$ & $=$ & $\cdots$ & \\
\hline
\end{tabular}


Table 5.--Chemical analyses of

\begin{tabular}{|c|c|c|c|c|c|c|}
\hline $\begin{array}{c}\text { DATE } \\
\text { OF } \\
\text { SAMPLE }\end{array}$ & $\begin{array}{l}\text { TOTAL } \\
\text { DDT } \\
\text { (UG/L) }\end{array}$ & $\begin{array}{l}\text { TOTAL } \\
\text { DI- } \\
\text { AZINON } \\
\text { (UG/L) }\end{array}$ & $\begin{array}{l}\text { TOTAL } \\
\text { DI- } \\
\text { ELDRIN } \\
\text { (UG/L) }\end{array}$ & $\begin{array}{l}\text { TOTAL } \\
\text { ENORIN } \\
\text { (UG/L) }\end{array}$ & $\begin{array}{l}\text { TOTAL } \\
\text { HEPTA- } \\
\text { CHLOR } \\
\text { (UG/L) }\end{array}$ & $\begin{array}{l}\text { TOTAL } \\
\text { HEPTA- } \\
\text { CHLOR } \\
\text { EPOXIDE } \\
\text { (UG/L) }\end{array}$ \\
\hline
\end{tabular}

76-04-01

76-04-01

$74-11-26$

$75-02-12$

75-03-20

75-05-06

$75-08-14$

$75-12-05$

$75-12-05$

$76-03-30$

76-04-16

$75-06-19$

$75-08-20$

$75-12-05$

$75-12-05$

75-06-19

$75-08-20$

75-12-05

$75-12-05$

$74-11-26$

$75-02-12$

$75-03-20$

75-05-07

$75-08-14$

$75-12-04$

$75-12-04$

76-03-30

$74-11-26$

$75-02-11$

75-03-20

75-05-06

$75-08-14$

$75-12-05$

75-12-05

76-04-01

76-04-16

$74-11-27$

$75-02-11$

$75-03-20$

75-05-07

\begin{tabular}{|c|c|}
\hline- & $=$ \\
\hline 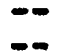 & $\because$ \\
\hline- & - \\
\hline- & - \\
\hline- & $\infty$ \\
\hline ND & ND \\
\hline- & $-\infty$ \\
\hline- & - \\
\hline$\cdots$ & $-\infty$ \\
\hline-- & - \\
\hline- & - \\
\hline ND & ND \\
\hline-- & -- \\
\hline$-\infty$ & -- \\
\hline-- & -- \\
\hline 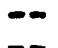 & $-\infty$ \\
\hline$\because$ & $=$ \\
\hline$\therefore$ & - \\
\hline & \\
\hline- & -- \\
\hline$\because$ & $=-$ \\
\hline$\ldots$ & $=$ \\
\hline- & -- \\
\hline- & -- \\
\hline- & $\infty$ \\
\hline$-\infty$ & $=$ \\
\hline$-\infty$ & $\Rightarrow$ \\
\hline$-\infty$ & $\cdots$ \\
\hline$=$ & -- \\
\hline$\ldots$ & $=$ \\
\hline- & -- \\
\hline-- & -- \\
\hline-- & - \\
\hline$=$ & 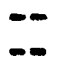 \\
\hline$\cdots$ & $-\infty$ \\
\hline$-\infty$ & -- \\
\hline
\end{tabular}

$=-$

$=$

ND

$--$

$-$

$-$

ND

$-\infty$

ND

-

$-$

$-$

$-$

$\cdots$

ND

$\infty$

$=$

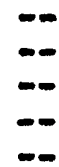

$-\infty$

$-\infty$

$-\infty$

$-$

$-$

$-$

$-\infty$

--
--
--
--
--
--

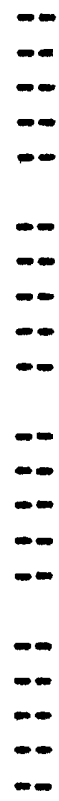

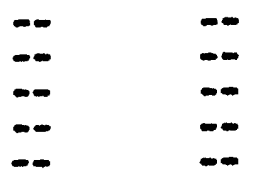

$-$

ND ND

ND $\quad-\infty$

- $\quad-$

$-$

$-$

$-$

ND

$-$

$-$

$-$

$-$

$-\infty$

$-$

$-$

$-$

$-$

$=$

$-$

$\cdots$

$-$

$-$

$-$

$-$

$-$

$-$

$-$

$-$

$-\infty$

$\because$

$-$

$-\infty$ 
water from wells--Continued

\begin{tabular}{|c|c|c|c|c|c|}
\hline $\begin{array}{l}\text { TOTAL } \\
\text { LINDANE } \\
\text { (UG/L) }\end{array}$ & $\begin{array}{l}\text { TOTAL } \\
\text { MALA- } \\
\text { THION } \\
\text { (UG/L) }\end{array}$ & $\begin{array}{l}\text { TOTAL } \\
\text { METHYL } \\
\text { PARA- } \\
\text { THION } \\
\text { (UG/L) }\end{array}$ & $\begin{array}{l}\text { TOTAL } \\
\text { PARA- } \\
\text { THION } \\
\text { (UG/L) }\end{array}$ & $\begin{array}{l}\text { TOTAL } \\
\text { PCB } \\
\text { (UG/L) }\end{array}$ & $\begin{array}{l}\text { TOTAL } \\
\text { TOX- } \\
\text { APHENE } \\
\text { (UG/L) }\end{array}$ \\
\hline$-\infty$ & -- & $-\infty$ & -- & $\cdots$ & $-\infty$ \\
\hline-- & - & - & $-\infty$ & $-\infty$ & $-\infty$ \\
\hline$-\infty$ & $-\infty$ & $-\infty$ & $\infty$ & $\infty$ & $\infty$ \\
\hline$-\infty$ & $-\infty$ & $-\infty$ & $-\infty$ & $-\infty$ & $-\infty$ \\
\hline$\infty$ & $\cdots$ & 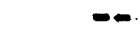 & -- & - & -- \\
\hline$-\infty$ & - & $-\infty$ & $-\infty$ & - & $-\infty$ \\
\hline ND & ND & ND & ND & ND & ND \\
\hline$-\infty$ & 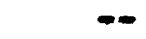 & $m$ & $-\infty$ & $\rightarrow$ & $\infty$ \\
\hline-- & $-\infty$ & $-\infty$ & $-\infty$ & $-\infty$ & $\infty$ \\
\hline$\infty$ & - & - & $-\infty$ & - & $-\infty$ \\
\hline-- & - & - & $-\infty$ & $-\infty$ & $-\infty$ \\
\hline$-\infty$ & $\cdots$ & - & $\rightarrow$ & 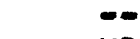 & $\infty$ \\
\hline ND & ND & ND & ND & ND & ND \\
\hline$-\infty$ & -- & -- & $-\infty$ & - & $\infty$ \\
\hline- & $\cdots$ & $\cdots$ & $-\infty$ & $\infty$ & $\infty$ \\
\hline$-\infty$ & - & $-\infty$ & $\infty$ & $-\infty$ & $-\infty$ \\
\hline$-\infty$ & - & $-\infty$ & -- & $-\infty$ & $\infty$ \\
\hline$-\infty$ & $-\infty$ & $\cdots$ & $-\infty$ & $-\infty$ & $-\infty$ \\
\hline-- & - & $-\infty$ & - & -- & $-\infty$ \\
\hline$-\infty$ & - & $-\infty$ & $-\infty$ & $-\infty$ & $=$ \\
\hline- & -- & -- & $-\infty$ & $\cdots$ & $\infty$ \\
\hline$-\infty$ & - & $-\infty$ & $-\infty$ & $-\infty$ & -- \\
\hline$-\infty$ & - & - & -- & $-\infty$ & $-\infty$ \\
\hline$-\infty$ & -- & $\cdots$ & - & $-\infty$ & $-\infty$ \\
\hline$-\infty$ & $-\infty$ & - & $-\infty$ & $\infty$ & $-\infty$ \\
\hline-- & -- & $-\infty$ & $-\infty$ & $=\infty$ & $-\infty$ \\
\hline$-\infty$ & - & -- & $-\infty$ & $-\infty$ & $-\infty$ \\
\hline$\infty$ & - & $-\infty$ & $-\infty$ & $-\infty$ & $-\infty$ \\
\hline$-\infty$ & -- & $-\infty$ & $\infty$ & $-\infty$ & $-\infty$ \\
\hline$-\infty$ & -- & - & $-\infty$ & $-\infty$ & $-\infty$ \\
\hline$-\infty$ & - & $-\infty$ & $\ldots$ & $-\cdots$ & $\infty$ \\
\hline$-\infty$ & $\cdots$ & $\infty$ & $-\infty$ & $-\infty$ & - \\
\hline$-\infty$ & $\cdots$ & $-\infty$ & $-\infty$ & - & $-\infty$ \\
\hline$-\infty$ & - & $-\infty$ & - & - & - \\
\hline$-\infty$ & $-\infty$ & $-\infty$ & $-\infty$ & $-\infty$ & $-\infty$ \\
\hline-- & - & -- & -- & $-\infty$ & - \\
\hline$-\infty$ & $-\infty$ & $-\infty$ & $-\infty$ & $-\infty$ & $-\infty$ \\
\hline$-\infty$ & $=$ & 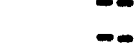 & $-\infty$ & $-\infty$ & $-\infty$ \\
\hline$\ldots$ & - & $=$ & $=$ & $\ldots$ & - \\
\hline
\end{tabular}




\begin{tabular}{|c|c|c|c|c|}
\hline $\begin{array}{c}\text { DATE } \\
\text { OF } \\
\text { SAMPLE }\end{array}$ & $\begin{array}{l}\text { DIS- } \\
\text { SOLVED } \\
\text { CAD- } \\
\text { MIUM } \\
\text { (CD) } \\
(U G / L)\end{array}$ & $\begin{array}{l}\text { DIS- } \\
\text { SOLVED } \\
\text { CHRO- } \\
\text { MIUM } \\
\text { (CR) } \\
(U G / L)\end{array}$ & $\begin{array}{l}\text { OIS- } \\
\text { SOLVED } \\
\text { COPPER } \\
(C U) \\
(U G / L)\end{array}$ & $\begin{array}{l}\text { DIS- } \\
\text { SOLVED } \\
\text { LEAD } \\
\text { (PB) } \\
\text { (UG/L) }\end{array}$ \\
\hline $\begin{array}{l}76-04-01 \\
76-04-01 \\
74-11-26 \\
75-02-12 \\
75-03-20\end{array}$ & $\begin{array}{r}-- \\
\text { ND } \\
1 \\
\text { ND } \\
--\end{array}$ & $\begin{array}{l}-- \\
\text { ND } \\
50 \\
10 \\
--\end{array}$ & $\begin{array}{l}-\infty \\
10 \\
\text { ND } \\
10 \\
--\end{array}$ & $\begin{array}{l}-2 \\
60 \\
10 \\
40 \\
--\end{array}$ \\
\hline $\begin{array}{l}75-05-06 \\
75-08-14 \\
75-12-05 \\
75-12-05 \\
76-03-30\end{array}$ & $\begin{array}{r}-- \\
\text { ND } \\
-- \\
\text { ND }\end{array}$ & $\begin{array}{l}-- \\
20 \\
\text { ND } \\
-- \\
\text { ND }\end{array}$ & $\begin{array}{l}-- \\
10 \\
10 \\
20\end{array}$ & $\begin{array}{l}-- \\
20 \\
\text { NO } \\
-\infty \\
80\end{array}$ \\
\hline $\begin{array}{l}76-04-16 \\
75-06-19 \\
75-08-20 \\
75-12-05 \\
75-12-05\end{array}$ & $\begin{array}{r}\text { NO } \\
1 \\
2 \\
--\end{array}$ & $\begin{array}{l}-- \\
30 \\
30 \\
\text { ND } \\
--\end{array}$ & $\begin{array}{l}-- \\
30 \\
60 \\
10 \\
--\end{array}$ & $\begin{array}{l}-- \\
\text { ND } \\
\text { ND } \\
--\end{array}$ \\
\hline $\begin{array}{l}75-06-19 \\
75-08-20 \\
75-12-05 \\
75-12-05 \\
74-11-26\end{array}$ & $\begin{array}{r}\text { ND } \\
\text { ND } \\
5 \\
-- \\
2\end{array}$ & $\begin{array}{l}10 \\
50 \\
30 \\
-- \\
20\end{array}$ & $\begin{array}{r}120 \\
30 \\
70 \\
-- \\
\text { NO }\end{array}$ & $\begin{array}{r}20 \\
60 \\
170 \\
-- \\
40\end{array}$ \\
\hline $\begin{array}{l}75-02-12 \\
75-03-20 \\
75-05-07 \\
75-08-14 \\
75-12-04\end{array}$ & $\begin{array}{r}1 \\
--- \\
2 \\
6\end{array}$ & $\begin{array}{l}\text { NO } \\
-- \\
-- \\
20 \\
\text { ND }\end{array}$ & $\begin{array}{l}\text { NO } \\
-- \\
20 \\
30\end{array}$ & $\begin{array}{l}60 \\
-- \\
-- \\
10\end{array}$ \\
\hline $\begin{array}{l}75-12-04 \\
76-03-30 \\
74-11-26 \\
75-02-11 \\
75-03-20\end{array}$ & $\begin{array}{r}15 \\
1 \\
2 \\
-.\end{array}$ & $\begin{array}{l}-- \\
30 \\
50 \\
20 \\
--\end{array}$ & $\begin{array}{l}-- \\
60 \\
\text { ND } \\
\text { ND } \\
--\end{array}$ & $\begin{array}{r}-- \\
340 \\
50 \\
30 \\
--\end{array}$ \\
\hline $\begin{array}{l}75-05-06 \\
75-08-14 \\
75-12-05 \\
75-12-05 \\
76-04-01\end{array}$ & $\begin{array}{c}-- \\
\text { ND } \\
-- \\
\text { NO }\end{array}$ & $\begin{array}{l}-- \\
30 \\
\text { ND } \\
-- \\
10\end{array}$ & $\begin{array}{l}-- \\
20 \\
\text { ND } \\
-- \\
10\end{array}$ & $\begin{array}{l}-- \\
\text { ND } \\
\text { ND } \\
-- \\
30\end{array}$ \\
\hline $\begin{array}{l}76-04-16 \\
74-11-27 \\
75-02-11 \\
75-03-20 \\
75-05-07\end{array}$ & $\begin{array}{r}-- \\
1 \\
-\infty \\
--\end{array}$ & $\begin{array}{l}-- \\
30 \\
20 \\
=- \\
--\end{array}$ & $\begin{array}{l}-- \\
\text { NO } \\
10 \\
-- \\
--\end{array}$ & $\begin{array}{l}-- \\
\text { ND } \\
40 \\
-- \\
--\end{array}$ \\
\hline
\end{tabular}


water from wezls--Continued

\begin{tabular}{|c|c|c|c|c|}
\hline $\begin{array}{c}\text { DIS- } \\
\text { SOLVED } \\
\text { MERCURY } \\
\text { (HG) } \\
(U G / L)\end{array}$ & $\begin{array}{l}\text { DIS- } \\
\text { SOLVED } \\
\text { NICKEL } \\
\text { (NI) } \\
\text { (UG/L) }\end{array}$ & $\begin{array}{l}\text { DIS- } \\
\text { SOLVED } \\
\text { ZINC } \\
\text { (ZN) } \\
\text { (UG/L) }\end{array}$ & $\begin{array}{l}\text { CODE } \\
\text { FOR } \\
\text { AGENCY } \\
\text { ANA- } \\
\text { LYZING } \\
\text { SAMPLE }\end{array}$ & $\begin{array}{c}\text { TOTAL } \\
\text { DEPTH } \\
\text { OF } \\
\text { WELL } \\
\text { (FT) }\end{array}$ \\
\hline $\begin{array}{l}-- \\
-\infty \\
-\infty \\
--\end{array}$ & $\begin{array}{l}- \\
\text { ND } \\
40 \\
20 \\
--\end{array}$ & $\begin{array}{l}20 \\
20 \\
80 \\
60 \\
--\end{array}$ & $\begin{array}{r}-0 \\
9999 \\
9999 \\
9999 \\
9999\end{array}$ & $\begin{array}{r}175 \\
175 \\
37 \\
37 \\
37\end{array}$ \\
\hline $\begin{array}{l}= \\
=- \\
=- \\
-\infty\end{array}$ & $\begin{array}{l}-- \\
10 \\
\text { ND } \\
=- \\
\text { ND }\end{array}$ & $\begin{array}{l}-5 \\
20 \\
90 \\
-- \\
80\end{array}$ & $\begin{array}{r}9999 \\
9999 \\
9999 \\
9999\end{array}$ & $\begin{array}{l}37 \\
37 \\
37 \\
37 \\
37\end{array}$ \\
\hline $\begin{array}{l}-\infty \\
-\infty \\
-\infty \\
-\infty\end{array}$ & $\begin{array}{l}- \\
30 \\
50 \\
\text { ND } \\
--\end{array}$ & $\begin{array}{l}-- \\
60 \\
40 \\
10 \\
--\end{array}$ & $\begin{array}{r}-0 \\
9999 \\
9999 \\
9999 \\
--\end{array}$ & $\begin{array}{l}37 \\
53 \\
53 \\
53 \\
53\end{array}$ \\
\hline $\begin{array}{l}=- \\
=- \\
=- \\
\text { ND }\end{array}$ & $\begin{array}{l}20 \\
70 \\
20 \\
-- \\
10\end{array}$ & $\begin{array}{r}40 \\
130 \\
60 \\
-- \\
30\end{array}$ & $\begin{array}{r}9999 \\
9999 \\
9999 \\
-0 \\
9999\end{array}$ & $\begin{array}{r}153 \\
153 \\
153 \\
153 \\
63\end{array}$ \\
\hline $\begin{array}{l}-\infty \\
-\infty \\
-\infty \\
-\infty\end{array}$ & $\begin{array}{l}40 \\
-- \\
-- \\
30 \\
50\end{array}$ & $\begin{array}{r}80 \\
-0 \\
-- \\
60 \\
120\end{array}$ & $\begin{array}{l}9999 \\
9999 \\
9999 \\
9999 \\
9999\end{array}$ & $\begin{array}{l}63 \\
63 \\
63 \\
63 \\
63\end{array}$ \\
\hline $\begin{array}{l}-\infty \\
-\infty \\
-\infty \\
--\end{array}$ & $\begin{array}{l}-\infty \\
80 \\
\text { ND } \\
20 \\
-\infty\end{array}$ & $\begin{array}{r}150 \\
4000 \\
7630 \\
=-\end{array}$ & $\begin{array}{r}-0 \\
9999 \\
9999 \\
9999 \\
9999\end{array}$ & $\begin{array}{l}63 \\
63 \\
150 \\
150 \\
150\end{array}$ \\
\hline $\begin{array}{l}-\infty \\
-\infty \\
=- \\
-\infty \\
-\infty\end{array}$ & $\begin{array}{l}-- \\
\text { ND } \\
\text { ND }\end{array}$ & $\begin{array}{r}-0 \\
3500 \\
760 \\
-0 \\
440\end{array}$ & $\begin{array}{r}9999 \\
9999 \\
9999 \\
9999\end{array}$ & $\begin{array}{l}150 \\
150 \\
150 \\
150 \\
150\end{array}$ \\
\hline 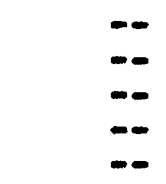 & $\begin{array}{l}-0 \\
10 \\
30 \\
--\end{array}$ & $\begin{array}{r}-- \\
120 \\
120 \\
--\end{array}$ & $\begin{array}{r}9999 \\
9999 \\
9999 \\
9999\end{array}$ & $\begin{array}{r}150 \\
53 \\
53 \\
53 \\
53\end{array}$ \\
\hline
\end{tabular}




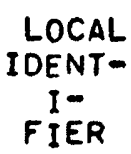

SC00506506CDD

SC00506506DBC3

SC00506508BC8

SC00506509ACD

SC005065098AA

SC00506509DDA

$S C 005065060 B C 1$

SC005065060BC2

$$
\text { SCO05065098AA }
$$

SC00506509D0A

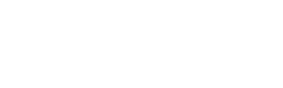

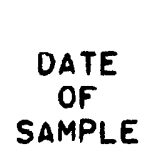

TOTAL DEPTH OF WELL (FT)
DISSOLVED IRON (FE) (UG/L) 
water from wells--Continued

$\begin{array}{cccccc}\text { DIS- } & \text { DIS- } & \text { DIS- } & & \text { DIS- } \\ \text { SOLVED } & & \text { SOLVED } & \\ \text { SOLVED } & \text { SOLVED } & \text { MAG- } & \text { DIS- } & \text { PO- } & \\ \text { MAN- } & \text { CAL- } & \text { NE- } & \text { SOLVED } & \text { TAS- } & \text { BICAR- } \\ \text { GANESE } & \text { CIUM } & \text { SIUM } & \text { SODIUM } & \text { SIUM } & \text { BONATE } \\ \text { (MN) } & \text { (CA) } & \text { (MG) } & \text { (NA) } & \text { (K) } & \text { (HCO3) } \\ (U G / L) & (M G / L) & \text { (MG/L) } & \text { (MG/L) } & (M G / L) & (M G / L)\end{array}$

\begin{tabular}{|c|c|c|c|c|c|}
\hline $\begin{array}{l}930 \\
950\end{array}$ & $\begin{array}{l}148 \\
277\end{array}$ & 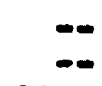 & $\begin{array}{l}110 \\
104\end{array}$ & $\begin{array}{l}17 \\
13\end{array}$ & $\begin{array}{l}229 \\
230\end{array}$ \\
\hline 840 & $228^{--}$ & $\begin{array}{l}38 \\
47\end{array}$ & $115^{--}$ & $14^{--}$ & 245 \\
\hline$-\infty$ & $=$ & $-\infty$ & $-\infty$ & - & $\infty$ \\
\hline $\begin{array}{l}20 \\
40\end{array}$ & $\begin{array}{l}241 \\
209\end{array}$ & $-\infty$ & $\begin{array}{l}310 \\
282\end{array}$ & $\begin{array}{l}19 \\
21\end{array}$ & $\begin{array}{l}214 \\
135\end{array}$ \\
\hline $\begin{array}{l}\text { ND } \\
20\end{array}$ & $\begin{array}{c}265^{--} \\
11\end{array}$ & $\begin{array}{r}8.4 \\
-0 \\
--\end{array}$ & $\begin{array}{l}160^{--} \\
118^{-0}\end{array}$ & $\begin{array}{l}33 \\
32\end{array}$ & $\begin{array}{l}\text { ND } \\
24\end{array}$ \\
\hline ND & 234 & $-\infty$ & 280 & 29 & 132 \\
\hline $\begin{array}{l}-\infty \\
10 \\
10 \\
\text { ND }\end{array}$ & $\begin{array}{l}183^{--} \\
362^{--}\end{array}$ & $\begin{array}{l}: 1 \\
: 3 \\
--\end{array}$ & $\begin{array}{l}265^{-0} \\
191\end{array}$ & $18^{--}$ & $\begin{array}{l}-\infty \\
\text { ND } \\
\text { ND }\end{array}$ \\
\hline $\begin{array}{l}30 \\
N D \\
10 \\
10\end{array}$ & $\begin{array}{l}330 \\
249 \\
260 \\
157\end{array}$ & 7.0 & $\begin{array}{l}212 \\
250 \\
290 \\
265\end{array}$ & $\begin{array}{l}25 \\
19 \\
11 \\
18\end{array}$ & $\begin{array}{r}71 \\
40 \\
47 \\
526\end{array}$ \\
\hline$-\infty$ & $-\infty$ & $-\infty$ & $-\infty$ & -- & - \\
\hline $\begin{array}{r}40 \\
80 \\
360 \\
1070 \\
-0\end{array}$ & $\begin{array}{l}51 \\
200 \\
200 \\
217 \\
203\end{array}$ & $\begin{array}{l}= \\
= \\
-\end{array}$ & $\begin{array}{r}67 \\
25 \\
127 \\
122 \\
74\end{array}$ & $\begin{array}{c}3.3 \\
15 \\
15 \\
6.7 \\
-\end{array}$ & $\begin{array}{r}162 \\
359 \\
261 \\
286 \\
--\end{array}$ \\
\hline $\begin{array}{l}700 \\
800\end{array}$ & $\begin{array}{l}115 \\
205\end{array}$ & $=$ & $\begin{array}{r}85 \\
106\end{array}$ & $\begin{array}{l}20 \\
14\end{array}$ & $\begin{array}{l}283 \\
281\end{array}$ \\
\hline$-\infty$ & - & 26 & $-\infty$ & $-\infty$ & - \\
\hline 1100 & $110^{--}$ & 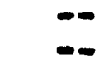 & $102^{-\infty}$ & $15^{--}$ & 364 \\
\hline 2000 & 141 & $\infty$ & 46 & 8.2 & 289 \\
\hline- & 142 & $\infty$ & 34 & - & - \\
\hline $\begin{array}{l}2880 \\
2300\end{array}$ & $\begin{array}{r}118 \\
14\end{array}$ & $=$ & $\begin{array}{l}55 \\
47\end{array}$ & $\begin{array}{l}15 \\
12\end{array}$ & $\begin{array}{l}344 \\
309\end{array}$ \\
\hline$=$ & - & 16 & - & $-\infty$ & - \\
\hline $\begin{array}{l}1100 \\
1010\end{array}$ & $115^{--}$ & 15 & $41^{--}$ & 9.0 & $37 \overline{7}$ \\
\hline
\end{tabular}




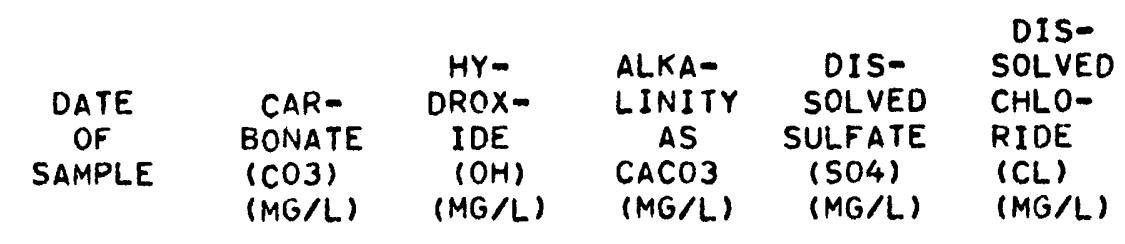

$75-08-14$

$75-12-08$

ND

ND

$75-12-08$

$76-03-30$

$76-04-16$

ND

$75-08-20$

$75-12-05$

$75-12-05$

$75-06-19$

$75-08-20$

ND

$75-12-05$

$75-12-05$

$76-04-02$

76-04-02

$75-06-19$

386

250

300

ND

$\begin{array}{lrc}\text { ND } & 188 & 890 \\ \text { ND } & 189 & 920 \\ -- & -- & - \\ \text { ND } & 201 & 1000 \\ -- & - & \end{array}$

37

36

75-08-20

75-12-05

$75-12-05$

76-04-02

$74-10-11$

ND

$-\infty$

18

48
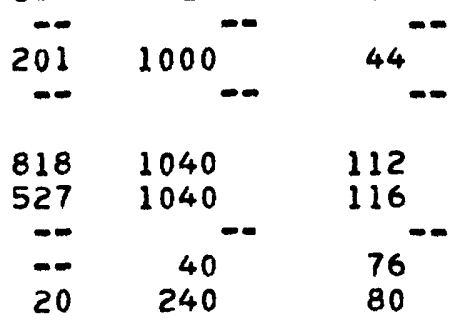

$75-02-13$

$75-08-15$

$74-11-25$

75-02-11

75-05-05

ND

ND

ND

1320

ND

20

NO

NO

$108 \quad 1100$

$-$

60

163

$=1120^{\circ}$

112

112
116

ND

ND

$-$

1050

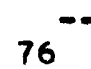

80

75-08-15

$75-12-04$

75-12-04

$76-04-16$

$74-11-25$

ND

ND

58

1100

$33 \quad 1280$

1100

ND

39

1020

96
104
104

ND

431
$-\infty$

1020

99

90

95

100

-

ND

ND

133

- 22

ND

133
294

$-\quad 147$

$\rightarrow$

214

$-$

235

$-$

58

$-$

--

$-$

54

ND

ND

232

490

230

ND

$-$

$-$

ND

$-$

$-$

299

550

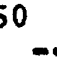

52

75-02-11

75-05-05

ND

$-\infty$

237

$-$

ND

$75-08-15$
$75-12-07$

ND

ND

$-$

282

253

ND

-.

$155^{--}$

53

$-$

$76-03-31$

76-03-31

ND

ND

309

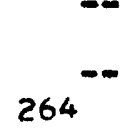

50

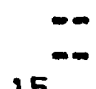

15

18

16

19

21

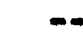


water from wezls--Continued

DIS-

SOLVED

FLUO-

RIDE

(F)

DIS-

SOLVED

NITRATE

DIS DOTAL DIS-

TOTAL SOLVED SOLVEO

(MG)

$$
\text { (N) }
$$

NITRATE NITRATE

(N)

NITRITE

(NO2)

$(M G / L) \quad(M G / L) \quad(M G / L) \quad(M G / L):(M G / L)$

NITRITE

(NO3) (NO3) (N)

$=$

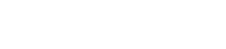

$-0.01$

$-\infty \quad 00$

$\therefore .00$

$-.01$

$-0.00$

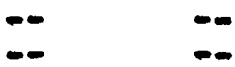

$\ldots .01$

$-.01$

$-01$

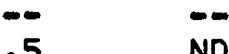

.5

$\infty$

$-.00$

$-\quad 5.5$

$-\infty .00$

$-\infty \quad 00$

$=00$

$-. \quad .02$

$-0.00$

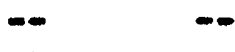

$\ddot{-2} \quad-\infty$

.23

$\Rightarrow \quad .00$

$\ldots \quad .05$

-. $\quad .00$

$-\infty \quad 0$

$=02$
-- 22

$-0.42$

$=16^{-\infty}$

$-\infty$

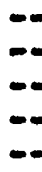

-

$\ldots$

$\ldots$

$-$

$\because$

$\ddot{-}$

$\because$

$\because$

$\because$

$\because$

$\because$

$\because$

$-$

$=1.0$

- $\quad .02$

$\ldots \quad .20$

- .02

$-$

$=$

NO
01

.10

.01

$-$

ND

-

.11
.01
.02
.02
.00
-0
.00
.00

$-$

$-\infty$

$-$

.01

.00

$-1$

.01

.00

.09

$-$

$-$

$-$

$-$

.00

$-$

.00

$-$ $-\infty$
--
--
--
-

$-\infty$

$-$

ND

.04

08

.08

-02
$-\quad 0.02$

.01

.02

$-\infty \quad$ ND

$-\infty$

--

$-$

ND

.04

$-\quad .01$

$-\infty$

.03

.02

$-$

$-$

ND

$-$

. 30

ND

.02

ND

ND

ND

$-\infty$
-

ND

$\begin{array}{ll}- & .02 \\ - & \text { ND }\end{array}$

-- NO

$\Longrightarrow 01$

$-$

$\ldots$

$-$

ND 


$\begin{array}{cccccc} & \text { DIS- } & & & \text { DIS- } & \text { DIS- } \\ & \text { SOLVED } & & & \text { SOLVED } & \text { SOLVED } \\ \text { AMMONIA } & & \text { DIS } & \text { ORGANIC } & \text { KJELL } \\ \text { DATE } & \text { NITRO- } & \text { TOTAL } & \text { SOLVED } & \text { NITRO- } & \text { NITRO- } \\ \text { OF } & \text { GEN } & \text { AMMONIA } & \text { AMMONIA } & \text { GEN } & \text { GEN } \\ \text { SAMPLE } & \text { (N) } & (N H 4) & (N H 4) & (N) & (N) \\ & (M G / L) & (M G / L) & (M G / L) & (M G / L) & (M G / L)\end{array}$

$75-08-14$
$75-12-08$
$75-12-08$
$76-03-30$
$76-04-16$

$$
-
$$

$75-08-20$

$75-12-05$

$75-12-05$

$75-06-19$

$75-08-20$

$75-12-05$

$75-12-05$

$76-04-02$

$76-04-02$

75-06-19

$75-08-20$

$75-12-05$

$75-12-05$

$76-04-02$

$74-10-11$

$75-02-13$

$75-08-15$

$74-11-25$

$75-02-11$

75-05-05

75-08-15

75-12-04

75-12-04

$76-04-16$

$74-11-25$

75-02-11

$75-05-05$

$75-08-15$

75-12-07

75-12-07

$76-03-31$

76-03-31$$
.10
$$

ND

ND

.20

.20

$--$

.60

ND

.10

$-$

.20

.20

.20

.20

.24

ND

-

NO

.40

ND

.30

ND

.10

ND

$-$

ND

ND

ND

.10

ND

-

-

ND

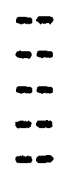

$\therefore .52$

$\begin{array}{ll}-\infty & .39 \\ - & -\infty\end{array}$

$\because$

$\because$

$-$

$-$

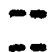

$-$

$-$

$-$

$-$

$=$

$-$

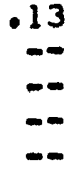

.26

.26

$-0$

.77

$--$

.13

-

.26

.26

.26

.26

.31

-.

$-$

-

.13

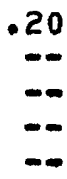

1.2

.40

$-$

.30

--

.60

$-\infty$

.40

.10

1.7

.30

.43

$-$

$-$

$-$

$-$

.30

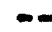

.10

$-$

$-\infty$

-

$\infty$

.13

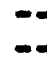

.30

$-\infty$

$-$

$-$

$-\infty$
.30

.10

$-$

.10

-

1.4

.60

$--$

.90

.30

.70

$-$

$-$

.60

.30

. 9

.50

.67

.10

-

.20

.

.40

.60

ND

.20

.40

$-$

3.6

1.6

ND

.40

.20

--

$-$ 
water from wells--Continued

\begin{tabular}{|c|c|c|c|c|c|c|}
\hline $\begin{array}{l}\text { DIS- } \\
\text { SOLVED } \\
\text { ORTHO. } \\
\text { PHOS- } \\
\text { PHOQRUS } \\
\text { (P) } \\
\text { (MG/L) }\end{array}$ & $\begin{array}{l}\text { DIS- } \\
\text { SOLVED } \\
\text { ORTHO } \\
\text { PHOS- } \\
\text { PHATE } \\
\text { (PO4) } \\
\text { (MG/L) }\end{array}$ & $\begin{array}{l}\text { DIS- } \\
\text { SOLVED } \\
\text { SOLIDS } \\
\text { (RESI- } \\
\text { DUE AT } \\
180^{\circ} \mathrm{C} \text { ) } \\
(M G / L)\end{array}$ & $\begin{array}{l}\text { OIS- } \\
\text { SOLVED } \\
\text { SOLIDS } \\
\text { (RESI- } \\
\text { OUE AT } \\
105^{\circ} \mathrm{C} \text { ) } \\
\text { (MG/L) }\end{array}$ & $\begin{array}{l}\text { DIS- } \\
\text { SOLVED } \\
\text { SOLIDS } \\
\text { (SUM OF } \\
\text { CONSTI- } \\
\text { TUENTS) } \\
\text { (MG/L) }\end{array}$ & $\begin{array}{l}\text { HARD- } \\
\text { NESS } \\
\text { (CA,MG) } \\
\text { (MG/L) }\end{array}$ & $\begin{array}{l}\text { NON- } \\
\text { CAR- } \\
\text { BONATE } \\
\text { HARD- } \\
\text { NESS } \\
\text { (MG/L) }\end{array}$ \\
\hline $\begin{array}{l}.01 \\
.01\end{array}$ & $\begin{array}{l}.03 \\
.03\end{array}$ & $=$ & $\begin{array}{l}1780 \\
1770\end{array}$ & $\therefore$ & $\begin{array}{r}1010 \\
994\end{array}$ & $\begin{array}{l}820 \\
810\end{array}$ \\
\hline- & $-\infty$ & $-\infty$ & $-\infty$ & $\infty$ & $=-$ & $-\infty$ \\
\hline NO & -- & $-\infty$ & 2020 & - & 1510 & 1300 \\
\hline$-\infty$ & - & $-\infty$ & $-\infty$ & - & - & $\infty$ \\
\hline $\begin{array}{r}.01 \\
\text { ND }\end{array}$ & .03 & $=$ & $\begin{array}{l}1840 \\
1620\end{array}$ & $\Longrightarrow$ & $\begin{array}{l}645 \\
646\end{array}$ & $120^{0}$ \\
\hline $\begin{array}{l}.0 \\
.02 \\
.04\end{array}$ & $\begin{array}{l}.0 \\
.06 \\
.12\end{array}$ & $=$ & $\begin{array}{l}152 \\
590\end{array}$ & $-\infty$ & $\begin{array}{r}983 \\
66\end{array}$ & -2 \\
\hline ND & - & - & 1830 & - & 697 & 590 \\
\hline$-\infty$ & - & $-\infty$ & $-\infty$ & - & $\ldots$ & $-\infty$ \\
\hline$-\overline{N D}$ & $\because$ & $=$ & 2000 & 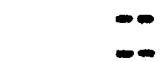 & 784 & $\therefore$ \\
\hline $\begin{array}{l}\text { NO } \\
\text { ND }\end{array}$ & 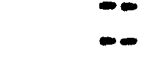 & $=$ & $\begin{array}{l}2000 \\
2050\end{array}$ & $-\infty$ & 851 & $=$ \\
\hline $\begin{array}{r}.03 \\
\text { ND } \\
.01 \\
\text { ND }\end{array}$ & $\begin{array}{r}.09 \\
.03 \\
.03 \\
-\infty\end{array}$ & $\begin{array}{l}-\infty \\
-\infty \\
-\infty\end{array}$ & $\begin{array}{r}1960 \\
1890 \\
1890\end{array}$ & 1800 & $\begin{array}{l}765 \\
700 \\
680 \\
692\end{array}$ & $\begin{array}{l}710 \\
670 \\
640 \\
260\end{array}$ \\
\hline$-\infty$ & $-\infty$ & $-\infty$ & 291 & $-\infty$ & $\ldots$ & $-\infty$ \\
\hline $\begin{array}{r}\text { ND } \\
.84 \\
.36 \\
8.0\end{array}$ & $\begin{array}{l}2.6^{-0} \\
1.1 \\
25\end{array}$ & $=$ & $\begin{array}{l}376 \\
1240 \\
1360 \\
1260\end{array}$ & $\begin{array}{l}-\infty \\
-\infty \\
-\infty\end{array}$ & $\begin{array}{l}138 \\
738 \\
797 \\
711\end{array}$ & $\begin{array}{r}5 \\
440 \\
580 \\
480\end{array}$ \\
\hline$-\infty$ & - & $-\infty$ & 1228 & $-\infty$ & $-\infty$ & - \\
\hline $\begin{array}{l}\text { ND } \\
.01\end{array}$ & .03 & $=$ & $\begin{array}{l}1240 \\
1160\end{array}$ & $=$ & $\begin{array}{l}700 \\
544\end{array}$ & $\begin{array}{l}470 \\
310\end{array}$ \\
\hline- & $=$ & -- & $-\infty$ & - & $\Longrightarrow$ & $-\infty$ \\
\hline $4 . \overline{8}^{-}$ & $15^{-}$ & $=$ & 65 & 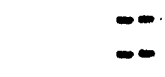 & 440 & 150 \\
\hline 2.2 & 6.7 & - & 626 & - & 421 & 180 \\
\hline$\rightarrow$ & & -- & 547 & & 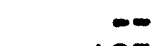 & - \\
\hline $\begin{array}{r}\text { ND } \\
.01\end{array}$ & .03 & 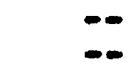 & $\begin{array}{l}704 \\
701\end{array}$ & 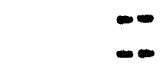 & $\begin{array}{l}437 \\
476\end{array}$ & $\begin{array}{l}160 \\
220\end{array}$ \\
\hline$\cdots$ & $-\infty$ & $-\infty$ & $-\infty$ & $\ldots$ & $-\infty$ & $-\infty$ \\
\hline - & 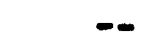 & $-\infty$ & -- & $\rightarrow$ & - & $-\infty$ \\
\hline NO & $-\infty$ & $-\infty$ & 685 & - & 452 & 140 \\
\hline
\end{tabular}




\begin{tabular}{|c|c|c|c|c|c|c|c|}
\hline $\begin{array}{l}\text { DATE } \\
\text { OF } \\
\text { SAMPLE }\end{array}$ & $\begin{array}{l}\text { SPE- } \\
\text { CIF IC } \\
\text { CON- } \\
\text { DUCT- } \\
\text { ANCE } \\
\text { (MICRO- } \\
\text { MHOS) }\end{array}$ & $\begin{array}{c}\text { PH } \\
\text { (UNITS) }\end{array}$ & $\begin{array}{l}\text { TEMPER- } \\
\text { ATURE } \\
\text { (DEG C) }\end{array}$ & $\begin{array}{l}\text { CARBON } \\
\text { DIOXIDE } \\
(\text { COLL) } \\
\text { (MG/L) }\end{array}$ & $\begin{array}{l}\text { FECAL } \\
\text { COLI- } \\
\text { FORM } \\
\text { (COL. } \\
\text { PER } \\
100 \mathrm{ML})\end{array}$ & $\begin{array}{l}\text { STR } \\
\text { TOCO } \\
\text { ICO } \\
\text { ONI } \\
\text { PE } \\
100\end{array}$ & $\begin{array}{l}\text { REP- } \\
\text { OCCI } \\
\text { OL- } \\
\text { IES } \\
\text { R } \\
\text { MLI }\end{array}$ \\
\hline $\begin{array}{l}75-08-14 \\
75-12-08 \\
75-12-08 \\
76-03-30 \\
76-04-16\end{array}$ & $\begin{array}{r}2500 \\
2400 \\
2400 \\
2175 \\
\ldots\end{array}$ & $\begin{array}{l}7.1 \\
6.8 \\
6.8 \\
6.9 \\
. .\end{array}$ & $\begin{array}{r}12.0 \\
9.5 \\
9.5 \\
10.0 \\
-.-\end{array}$ & $\begin{array}{l}29 \\
58 \\
49 \\
=-\end{array}$ & $\begin{array}{l}-\infty \\
-\infty \\
-\infty \\
-\infty\end{array}$ & & $\begin{array}{l}-\infty \\
-\infty \\
-\infty\end{array}$ \\
\hline $\begin{array}{l}75-08-20 \\
75-12-05 \\
75-12-05 \\
75-06-19 \\
75-08-20\end{array}$ & $\begin{array}{r}3100 \\
2800 \\
2800 \\
>8000 \\
1050\end{array}$ & $\begin{array}{r}10.5 \\
9.3 \\
9.3 \\
11.2 \\
9.6\end{array}$ & $\begin{array}{l}15.5 \\
13.0 \\
13.0 \\
14.0 \\
13.5\end{array}$ & $\begin{array}{l}.1 \\
.5 \\
-. . \\
. . \\
.0\end{array}$ & $\begin{array}{l}=- \\
=- \\
-- \\
--\end{array}$ & & $=$ \\
\hline $\begin{array}{l}75-12-05 \\
75-12-05 \\
76-04-02 \\
76-04-02 \\
75-06-19\end{array}$ & $\begin{array}{l}3000 \\
3000 \\
2600 \\
2600 \\
3750\end{array}$ & $\begin{array}{l}11.4 \\
11.4 \\
11.1 \\
11.1 \\
11.2\end{array}$ & $\begin{array}{r}9.5 \\
9.5 \\
14.5 \\
14.5 \\
15.0\end{array}$ & $\begin{array}{l}\because 0 \\
-- \\
=- \\
-\infty\end{array}$ & $\begin{array}{l}-\infty \\
-\infty \\
-\infty \\
-\infty\end{array}$ & & - \\
\hline $\begin{array}{l}5-08-20 \\
5-12-05 \\
5-12-05 \\
6-04-02 \\
4-10-11\end{array}$ & $\begin{array}{r}2900 \\
2700 \\
2700 \\
2400 \\
=-\end{array}$ & $\begin{array}{r}7.8 \\
7.9 \\
7.9 \\
8.7 \\
-.-\end{array}$ & $\begin{array}{r}14.5 \\
11.0 \\
11.0 \\
15.5 \\
. .\end{array}$ & $\begin{array}{r}1.8 \\
.8 \\
.9 \\
1.7 \\
-.-\end{array}$ & $\begin{array}{l}-- \\
-\infty \\
-\infty \\
--\end{array}$ & & 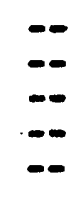 \\
\hline$\left\{\begin{array}{l}-02-13 \\
-08-15 \\
-11-25 \\
-02-11 \\
-05-05\end{array}\right.$ & $\begin{array}{l}550 \\
1750 \\
1900 \\
1700 \\
1800\end{array}$ & $\begin{array}{l}7.5 \\
7.4 \\
7.2 \\
6.9 \\
7.5\end{array}$ & $\begin{array}{l}15.5 \\
13.5 \\
12.0 \\
10.0 \\
11.0\end{array}$ & $\begin{array}{l}8.2 \\
23 \\
26 \\
58 \\
\ldots\end{array}$ & $\begin{array}{c}0 \\
-- \\
--\end{array}$ & & $\begin{array}{r}104 \\
-- \\
-- \\
-2\end{array}$ \\
\hline $\begin{array}{l}-08-15 \\
-12-04 \\
-12-04 \\
-04-16 \\
-11-25\end{array}$ & $\begin{array}{r}1850 \\
1700 \\
1700 \\
-- \\
1025\end{array}$ & $\begin{array}{l}7.6 \\
7.6 \\
7.6 \\
-.- \\
7.3\end{array}$ & $\begin{array}{r}11.5 \\
11.5 \\
11.5 \\
-.5 \\
11.5\end{array}$ & $29^{11}$ & $\begin{array}{l}-\infty \\
-\infty \\
-\infty \\
-\infty\end{array}$ & & $\begin{array}{c}-\infty \\
-- \\
-\infty\end{array}$ \\
\hline $\begin{array}{l}75-02-11 \\
75-05-05 \\
75-08-15 \\
75-12-07 \\
75-12-07\end{array}$ & $\begin{array}{r}975 \\
950 \\
1100 \\
1100 \\
1100\end{array}$ & $\begin{array}{l}6.8 \\
7.3 \\
7.6 \\
6.9 \\
6.9\end{array}$ & $\begin{array}{l}6.0 \\
10.5 \\
16.5 \\
11.0 \\
11.0\end{array}$ & $\begin{array}{l}73 \\
14^{--} \\
62^{--}\end{array}$ & $\begin{array}{l}1 \\
-- \\
-\therefore \\
--\end{array}$ & & $\begin{array}{l}1 \\
-- \\
-\infty\end{array}$ \\
\hline $\begin{array}{l}-03-31 \\
-03-31\end{array}$ & $\begin{array}{l}1000 \\
1000\end{array}$ & $\begin{array}{l}7.3 \\
7.3\end{array}$ & $\begin{array}{l}8.0 \\
8.0\end{array}$ & $30^{--}$ & $=$ & & $=$ \\
\hline
\end{tabular}


water from wezls--Continued

$$
\begin{array}{cccccc}
\text { CYANIDE } & \text { PHENOLS } & \text { TOTAL } & \begin{array}{c}
\text { TOTAL } \\
\text { CHLOR- }
\end{array} & \text { TOTAL } & \text { TOTAL } \\
(C N) & & \text { TOLRIN } & \text { DANE } & \text { DOD } & \text { DDE } \\
(M G / L) & (U G / L) & \text { (UG/L) } & (U G / L) & (U G / L) & (U G / L)
\end{array}
$$

\begin{tabular}{|c|c|c|c|c|}
\hline$-\infty$ & $-\infty$ & ND & ND & NO \\
\hline - & $-\infty$ & $\infty$ & $-\infty$ & $-\infty$ \\
\hline - & $\cdots$ & $\cdots$ & $-\infty$ & $-\infty$ \\
\hline & $=$ & $-\infty$ & $-\infty$ & $-\infty$ \\
\hline
\end{tabular}

$-\infty \quad-\infty$

$-$

$-\infty$

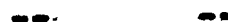

$-\infty$

$-\infty$

$-$

$-\infty$

$-$

$-\infty$

$-$

$-$

$-$

-

$-\infty$

$-$

$\infty$

$-\infty$

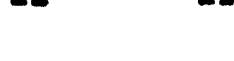

$-\infty$

$-\infty$

$-$

$-$

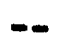

$-\infty$

$-\infty$

-

$-$

$-\infty$

$-$

$=$

$-\infty$

$-\infty$

$-\infty$

$-\infty$

$-$

$-\infty$

$-$

$-$

$-$

$-$

$-\infty$

$-\infty \quad-\infty$

$-\infty \quad+\infty$

$\infty$

$-$

ND

$-$

$-$

$\begin{array}{ll}\therefore & - \\ \therefore & \therefore \\ -\infty & \therefore\end{array}$

ND

$-\infty$

$-$

$-$

$-$

$-$

$-$

$-$

$\infty$

$=$

$-$

$-\infty$

$-\infty$

$-$

$-\infty$

$-$

-

$\infty$

$-\infty$

$\begin{array}{ll}-\infty & -\infty \\ -\infty & -\infty \\ -\infty & -\infty\end{array}$

$=$

$-$

$-$

$-$

$-$

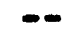

$-\infty$

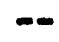

$\because$

$-$

$-$

$-$

$-$

$-\infty$

$\infty$

$-\infty$

$-$

$-\infty$

$-\infty$

$-$

$-$

$-$

$-$

$\because$

$=$

$=$

$\because$

$-\infty$ 
Table 5.--Chemical analyses of

\begin{tabular}{|c|c|c|c|c|c|c|}
\hline $\begin{array}{l}\text { DATE } \\
\text { OF } \\
\text { SAMPLE }\end{array}$ & $\begin{array}{l}\text { TOTAL } \\
\text { DOT } \\
\text { (UG/L) }\end{array}$ & $\begin{array}{l}\text { TOTAL } \\
\text { DI- } \\
\text { AZINON } \\
\text { (UG/L) }\end{array}$ & $\begin{array}{l}\text { TOTAL } \\
\text { DI- } \\
\text { ELDRIN } \\
\text { (UG/L) }\end{array}$ & $\begin{array}{l}\text { TOTAL } \\
\text { ENDRIN } \\
\text { (UG/L) }\end{array}$ & $\begin{array}{l}\text { TOTAL } \\
\text { HEPTA- } \\
\text { CHLOR } \\
\text { (UG/L) }\end{array}$ & $\begin{array}{l}\text { TOTAL } \\
\text { HEPTA- } \\
\text { CHLOR } \\
\text { EPOXIDE } \\
\text { (UG/L) }\end{array}$ \\
\hline
\end{tabular}

$75-08-14$

$75-12-08$

$75-12-08$

76-03-30

76-04-16

$75-08-20$

$75-12-05$

75-12-05

75-06-19

$75-08-20$

$75-12-05$

$75-12-05$

76-04-02

$76-04-02$

75-06-19

$75-08-20$

$75-12-05$

$75-12-05$

76-04-02

$74-10-11$

$75-02-13$

$75-08-15$

$74-11-25$

$75-02-11$

75-05-05

ND

ND

ND

--

--

$-$

$+\infty$

$-\infty$

$-$

$-$

$-\infty$

$-\infty$

$=$

--

$-$

$-$

$-$

$\begin{array}{lll}-\infty & -\infty\end{array}$

$-\infty$

$-$

$-$

(UG/L)

(UG/L) (UG/L)

$75-08-15$

$75-12-04$

$75-12-04$

$76-04-16$

$74-11-25$

$-$

$-$

$-$

$-$

$-\infty$

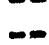

$-\infty$

$-$

$\infty$

--

ND

ND

ND

$\infty$

$-\infty$

-

$-$

$-$

$-$

$-$

$-\infty$

$-$

--

$-$

$\overline{-\infty}$

$-$

$-$

$-$

$-$

$-$

$-$

$-$

$-$

$-\infty$

--

$-$

$-$

$-$

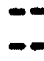

$\begin{array}{ll}-\infty & -\infty\end{array}$

$\infty$

$-$

$-$

$-$

$-$

$-$

$-$

$-$

-

$-$

-

$-$

$-$

$-$

--

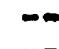

$=$

-.

$\ddot{-}$

$-$

$-$

$\because$

$\because$

$-$

$\because$

$=$

$-$

-:

$-$

$-$

$\because$

$=$

$-$

$-$

$-$

$-$

$-$

$-$

$-$

75-02-11

75-05-05

$75-08-15$

75-12-07

75-12-07

76-03-31

$76-03-31$

$\because$

$\because=$

$\because$

$\because$

$\because$

$-$

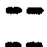

$\because$

..

$-\infty$

$\because$

$-$

$-$

$-$

$=$

$-$

-

$\infty$

$-$

$-\infty$

$-$

$-$

$-$

$-$

$-$

$-$

$-$

$-$

$-$

$-\infty$

$=$

$-\infty$

$-\infty$

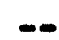

-.

$\because$

$-$

$\because$ 
water from wells--Continued

\begin{tabular}{|c|c|c|c|c|c|}
\hline $\begin{array}{l}\text { TOTAL } \\
\text { LINDANE } \\
\text { (UG/L) }\end{array}$ & $\begin{array}{l}\text { TOTAL } \\
\text { MALA- } \\
\text { THION } \\
\text { (UG/L) }\end{array}$ & $\begin{array}{l}\text { TOTAL } \\
\text { METHYL } \\
\text { PARA- } \\
\text { THION } \\
\text { (UG/L) }\end{array}$ & $\begin{array}{l}\text { TOTAL } \\
\text { PARA- } \\
\text { THION } \\
\text { (UG/L) }\end{array}$ & $\begin{array}{l}\text { TOTAL } \\
\text { PCB } \\
\text { (UG/L) }\end{array}$ & $\begin{array}{l}\text { TOTAL } \\
\text { TOX- } \\
\text { APHENE } \\
\text { (UG/L) }\end{array}$ \\
\hline ND & No & ND & ND & ND & ND \\
\hline$-\infty$ & $-\infty$ & $\infty$ & - & $-\infty$ & $=-$ \\
\hline$\ldots$ & -- & $-\infty$ & -- & $-\infty$ & $-\infty$ \\
\hline- & $\cdots$ & - & - & $\cdots$ & 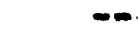 \\
\hline- & -- & - & $-\infty$ & $-\infty$ & $-\infty$ \\
\hline$-\infty$ & -- & $=-$ & $-\infty$ & $-\infty$ & $-\infty$ \\
\hline- & $-\infty$ & - & $-\infty$ & $-\infty$ & $-\infty$ \\
\hline- & $-\infty$ & $\infty$ & $-\infty$ & $-\infty$ & $-\infty$ \\
\hline- & $-\infty$ & $-\infty$ & $-\infty$ & $-\infty$ & $-\infty$ \\
\hline 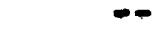 & - & $\infty$ & -- & $-\infty$ & $\cdots$ \\
\hline$-\infty$ & $-\infty$ & $\infty$ & $-\infty$ & $-\infty$ & $\infty$ \\
\hline- & $-\infty$ & $-\infty$ & $\infty$ & $\infty$ & $-\infty$ \\
\hline$-\infty$ & $-\infty$ & $\infty$ & - & $-\infty$ & - \\
\hline- & $=$ & $=$ & $=$ & $=-$ & 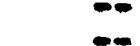 \\
\hline- & $-\infty$ & $-\infty$ & $-\infty$ & $+\infty$ & $-\infty$ \\
\hline- & - & $-\infty$ & $-\infty$ & $-\infty$ & $-\infty$ \\
\hline- & $-\infty$ & - & - & $-\infty$ & - \\
\hline-- & - & -- & -- & $-\infty$ & $-\infty$ \\
\hline$-\infty$ & $-\infty$ & - & -- & $-\infty$ & - \\
\hline$-\infty$ & -- & $-\infty$ & - & -- & $-\infty$ \\
\hline$\infty$ & - & $-\infty$ & $=-$ & $\cdots$ & $\infty$ \\
\hline- & - & -- & $-\infty$ & - & $-\infty$ \\
\hline$-\infty$ & $-\infty$ & $-\infty$ & $-\infty$ & -- & $=-$ \\
\hline$=$ & -- & - & $m$ & $\because$ & $\infty$ \\
\hline$\Rightarrow$ & $\cdots$ & $-\infty$ & $\cdots$ & $-\infty$ & $\infty$ \\
\hline$-\infty$ & $-\infty$ & $-\infty$ & - & $-\infty$ & $-\infty$ \\
\hline$\cdots$ & $\infty$ & - & $-\infty$ & - & $-\infty$ \\
\hline$-\infty$ & $-\infty$ & -- & $=$ & $-\infty$ & $-\infty$ \\
\hline$=$ & $=$ & 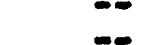 & $=$ & $-\infty$ & $-\infty$ \\
\hline- & $-\infty$ & $-\infty$ & $-\infty$ & $-\infty$ & - \\
\hline- & $\infty$ & $=-$ & - & $-\infty$ & $\infty$ \\
\hline 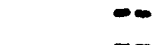 & $\infty$ & $-\infty$ & $-\infty$ & - & $-\infty$ \\
\hline 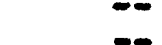 & $\cdots$ & - & -- & $\infty$ & $-\infty$ \\
\hline$\therefore$ & 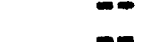 & $-\infty$ & $\because$ & - & 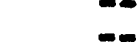 \\
\hline & & & & & \\
\hline$=$ & $-\infty$ & $-\infty$ & $-\infty$ & $-\infty$ & $=-$ \\
\hline
\end{tabular}


Table 5.--Chemical analyses of

\begin{tabular}{|c|c|c|c|c|}
\hline $\begin{array}{c}\text { DATE } \\
\text { OF } \\
\text { SAMPLE }\end{array}$ & $\begin{array}{l}\text { DIS- } \\
\text { SOLVED } \\
\text { CAD- } \\
\text { MIUM } \\
\text { (CD) } \\
(U G / L)\end{array}$ & $\begin{array}{l}\text { DIS- } \\
\text { SOLVED } \\
\text { CHRO- } \\
\text { MIUM } \\
\text { (CR) } \\
\text { (UG/L) }\end{array}$ & $\begin{array}{l}\text { DIS- } \\
\text { SOLVED } \\
\text { COPPER } \\
\text { (CU) } \\
\text { (UG/L) }\end{array}$ & $\begin{array}{l}\text { DIS- } \\
\text { SOLVED } \\
\text { LEAD } \\
\text { (PB) } \\
(U G / L)\end{array}$ \\
\hline $\begin{array}{l}75-08-14 \\
75-12-08 \\
75-12-08 \\
76-03-30 \\
76-04-16\end{array}$ & $\begin{array}{r}\text { ND } \\
7 \\
-- \\
9 \\
--\end{array}$ & $\begin{array}{l}40 \\
10 \\
-- \\
20 \\
--\end{array}$ & $\begin{array}{l}20 \\
\text { ND } \\
-- \\
30 \\
=-\end{array}$ & $\begin{array}{r}\text { ND } \\
80 \\
100 \\
--\end{array}$ \\
\hline $\begin{array}{l}75-08-20 \\
75-12-05 \\
75-12-05 \\
75-06-19 \\
75-08-20\end{array}$ & $\begin{array}{r}\text { ND } \\
2 \\
-\infty \\
\text { ND } \\
\text { ND }\end{array}$ & $\begin{array}{r}20 \\
10 \\
-0 \\
70 \\
120\end{array}$ & $\begin{array}{r}20 \\
20 \\
-0 \\
30 \\
320\end{array}$ & $\begin{array}{l}10 \\
\text { ND } \\
-- \\
10 \\
10\end{array}$ \\
\hline $\begin{array}{l}75-12-05 \\
75-12-05 \\
76-04-02 \\
76-04-02 \\
75-06-19\end{array}$ & $\begin{array}{l}5 \\
-- \\
-\infty \\
\text { ND } \\
\text { NO }\end{array}$ & $\begin{array}{l}10 \\
-- \\
20 \\
10\end{array}$ & $\begin{array}{l}20 \\
-0 \\
-0 \\
20 \\
10\end{array}$ & $\begin{array}{r}\text { ND } \\
-- \\
-- \\
120 \\
\text { NO }\end{array}$ \\
\hline $\begin{array}{l}75-08-20 \\
75-12-05 \\
75-12-05 \\
76-04-02 \\
74-10-11\end{array}$ & $\begin{array}{r}4 \\
7 \\
-- \\
\text { ND } \\
--\end{array}$ & $\begin{array}{l}10 \\
\text { ND } \\
-- \\
\text { ND } \\
--\end{array}$ & $\begin{array}{r}120 \\
10 \\
-- \\
20 \\
=-\end{array}$ & $\begin{array}{l}60 \\
\text { ND } \\
-- \\
60 \\
--\end{array}$ \\
\hline $\begin{array}{l}75-02-13 \\
75-08-15 \\
74-11-25 \\
75-02-11 \\
75-05-05\end{array}$ & $\begin{array}{r}1 \\
\text { ND } \\
2 \\
\text { ND } \\
--\end{array}$ & $\begin{array}{l}\text { ND } \\
10 \\
50 \\
30 \\
-\infty\end{array}$ & $\begin{array}{l}\text { ND } \\
70 \\
\text { ND } \\
\text { ND } \\
--\end{array}$ & $\begin{array}{l}20 \\
10 \\
\text { ND } \\
40 \\
-\infty\end{array}$ \\
\hline $\begin{array}{l}75-08-15 \\
75-12-04 \\
75-12-04 \\
76-04-16 \\
74-11-25\end{array}$ & $\begin{array}{r}\text { ND } \\
5 \\
-- \\
-- \\
\text { ND }\end{array}$ & $\begin{array}{l}40 \\
10 \\
-- \\
--\end{array}$ & $\begin{array}{l}80 \\
10 \\
-= \\
-- \\
\text { NO }\end{array}$ & $\begin{array}{l}30 \\
\text { ND } \\
-- \\
-- \\
\text { ND }\end{array}$ \\
\hline $\begin{array}{l}75-02-11 \\
75-05-05 \\
75-08-15 \\
75-12-07 \\
75-12-07\end{array}$ & $\begin{array}{l}\text { ND } \\
-- \\
\text { ND } \\
1 \\
--\end{array}$ & $\begin{array}{l}10 \\
-0 \\
40 \\
N D \\
--\end{array}$ & $\begin{array}{r}\text { ND } \\
-\infty \\
330 \\
\text { ND } \\
-\infty\end{array}$ & $\begin{array}{l}20 \\
-- \\
30 \\
40 \\
--\end{array}$ \\
\hline $\begin{array}{l}76-03-31 \\
76-03-31\end{array}$ & $\overline{\text { NO }}$ & -- & 10 & $\overrightarrow{20}$ \\
\hline
\end{tabular}




\begin{tabular}{|c|c|c|c|c|}
\hline $\begin{array}{l}\text { DIS- } \\
\text { SOLVED } \\
\text { MERCURY } \\
\text { (HG) } \\
\text { (UG/L) }\end{array}$ & $\begin{array}{l}\text { DIS- } \\
\text { SOLVED } \\
\text { NICKEL } \\
\text { (NI) } \\
(U G / L)\end{array}$ & $\begin{array}{l}\text { DIS- } \\
\text { SOLVED } \\
\text { ZINC } \\
\text { (ZN) } \\
(U G / L)\end{array}$ & $\begin{array}{l}\text { CODE } \\
\text { FOR } \\
\text { AGENCY } \\
\text { ANA- } \\
\text { LYZING } \\
\text { SAMPLE }\end{array}$ & $\begin{array}{c}\text { TOTAL } \\
\text { DEPTH } \\
\text { OF } \\
\text { WELL } \\
\text { (FT) }\end{array}$ \\
\hline $\begin{array}{l}-\infty \\
-\infty \\
-\infty \\
-\infty\end{array}$ & $\begin{array}{l}10 \\
30 \\
-- \\
10 \\
--\end{array}$ & $\begin{array}{r}600 \\
600 \\
-- \\
540 \\
--\end{array}$ & $\begin{array}{r}9999 \\
9999 \\
-- \\
9999 \\
--\end{array}$ & $\begin{array}{l}53 \\
53 \\
53 \\
53 \\
53\end{array}$ \\
\hline $\begin{array}{l}=- \\
=- \\
=- \\
=-\end{array}$ & $\begin{array}{l}40 \\
10 \\
-1 \\
20 \\
40\end{array}$ & $\begin{array}{l}20 \\
10 \\
-- \\
40 \\
40\end{array}$ & $\begin{array}{l}9999 \\
9999 \\
9999 \\
9999 \\
9999\end{array}$ & $\begin{array}{r}80 \\
80 \\
80 \\
177 \\
177\end{array}$ \\
\hline $\begin{array}{l}-\infty \\
=- \\
=- \\
--\end{array}$ & $\begin{array}{l}\text { ND } \\
-- \\
-0 \\
10 \\
40\end{array}$ & $\begin{array}{l}20 \\
-- \\
20 \\
10 \\
\text { ND }\end{array}$ & $\begin{array}{r}9999 \\
-- \\
9999 \\
9999\end{array}$ & $\begin{array}{l}177 \\
177 \\
177 \\
177 \\
244\end{array}$ \\
\hline $\begin{array}{l}-\infty \\
-\infty \\
-\infty \\
-\infty\end{array}$ & $\begin{array}{l}80 \\
10 \\
-- \\
\text { ND } \\
--\end{array}$ & $\begin{array}{r}30 \\
280 \\
-- \\
20 \\
--\end{array}$ & $\begin{array}{r}9999 \\
9999 \\
-0 \\
9999 \\
9999\end{array}$ & $\begin{array}{l}244 \\
244 \\
244 \\
244 \\
356\end{array}$ \\
\hline $\begin{array}{l}= \\
=- \\
-- \\
-- \\
--\end{array}$ & $\begin{array}{l}\text { ND } \\
10 \\
10 \\
10 \\
--\end{array}$ & $\begin{array}{r}70 \\
280 \\
100 \\
20 \\
--\end{array}$ & $\begin{array}{l}9999 \\
9999 \\
9999 \\
9999 \\
9999\end{array}$ & $\begin{array}{r}356 \\
19 \\
23 \\
23 \\
23\end{array}$ \\
\hline $\begin{array}{l}\ldots \\
\therefore \\
\therefore \\
--\end{array}$ & $\begin{array}{l}20 \\
20 \\
-- \\
-- \\
\text { ND }\end{array}$ & $\begin{array}{l}10 \\
90 \\
-- \\
-- \\
50\end{array}$ & $\begin{array}{r}9999 \\
9999 \\
- \\
9999\end{array}$ & $\begin{array}{l}23 \\
23 \\
23 \\
23 \\
10\end{array}$ \\
\hline $\begin{array}{l}= \\
=- \\
=\end{array}$ & $\begin{array}{l}10 \\
-- \\
\text { ND } \\
\text { ND } \\
--\end{array}$ & $\begin{array}{l}10 \\
-0 \\
70 \\
40 \\
--\end{array}$ & $\begin{array}{r}9999 \\
9999 \\
9999 \\
9999 \\
-\end{array}$ & $\begin{array}{l}10 \\
10 \\
10 \\
10 \\
10\end{array}$ \\
\hline$=$ & NO & $\begin{array}{r}130 \\
50\end{array}$ & $99 \overline{9}$ & $\begin{array}{l}10 \\
10\end{array}$ \\
\hline
\end{tabular}




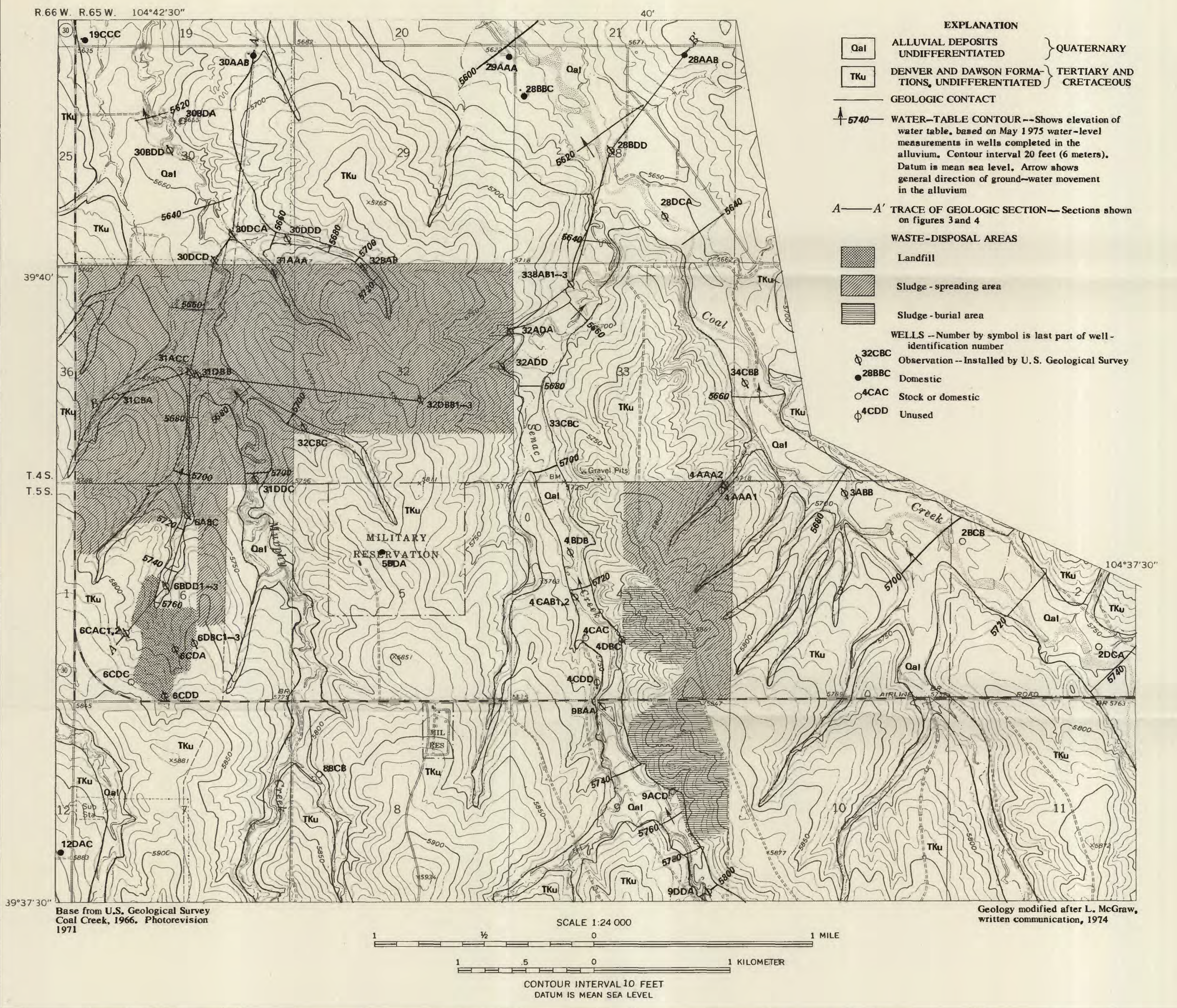

MAP SHOWING GEOLOGY, LOCATION OF WELLS AND GEOLOGIC SECTIONS, AND WATER-LEVEL CONTOURS IN ALLUVIUM FOR MAY 1975 NEAR A SEWAGE-SLUDGE RECYCLING SITE AND A LANDFILL NEAR DENVER, COLORADO 


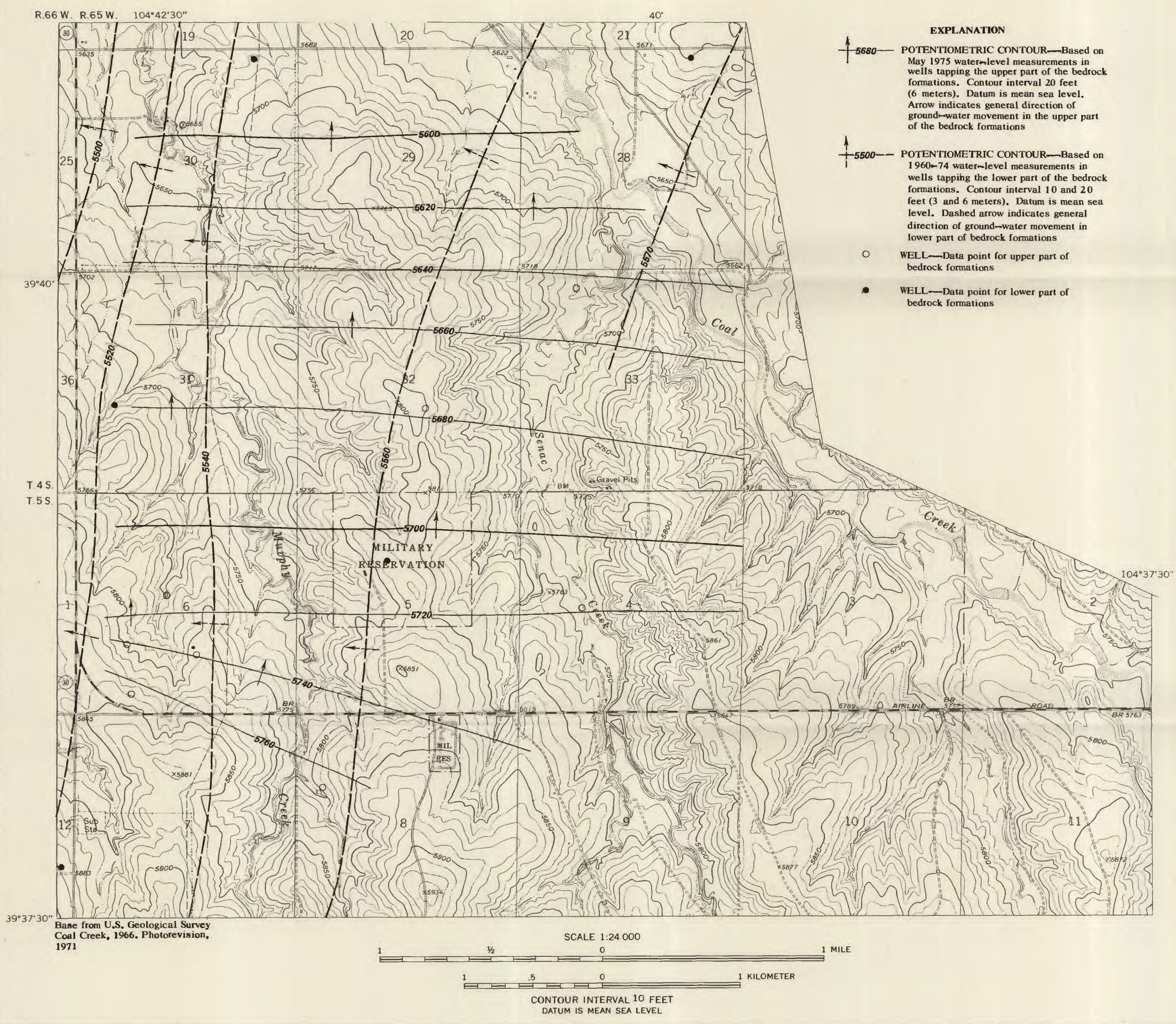

MAP SHOWING POTENTIOMETRIC CONTOURS FOR THE UPPER AND LOWER PARTS OF THE BEDROCK FORMATIONS NEAR A SEWAGE-SLUDGE RECYCLING SITE AND A LANDFILL NEAR DENVER, COLORADO 